$$
\begin{aligned}
& \ldots \\
& \text { - } \\
& \text { - } \\
& -\ldots \\
& \text { Spin-orbit-coupling induced } \\
& \text {... - lateral spin transport from } \\
& \text { first principles } \\
& \text { - }- \\
& \text { - } \\
& \text { - } \\
& \text { - } \\
& \text { - - - } \\
& \text { - }+ \\
& \text { - - - } \\
& \ldots \\
& \text { - } \\
& \text { - } \\
& \text { - - - } \\
& \text { - } \\
& \text { - } \\
& -\ldots \\
& \text { - } \\
& \text { - } \\
& \text { - } \\
& \text { - }
\end{aligned}
$$

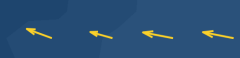

$$
\begin{aligned}
& \cdots+\cdots \\
& \text { Rohit Sasidharan Nair }
\end{aligned}
$$


SPIN-ORBIT-COUPLING INDUCED LATERAL SPIN TRANSPORT FROM FIRST PRINCIPLES

Rohit Sasidharan Nair 
This dissertation has been approved by:

Prof. Dr. Paul J. Kelly (promotor)

The work in this thesis was carried out in the Computational Materials Science group of the Faculty of Science and Technology (TNW) at the University of Twente. This work was financially supported by the "Nederlandse Organisatie voor Wetenschappelijk Onderzoek" (NWO) through the research programme of the former "Stichting voor Fundamenteel Onderzoek der Materie," (NWO-I, formerly FOM) and through the use of supercomputer facilities of NWO "Exacte Wetenschappen" (Physical Sciences). We acknowledge the funding from the Shell-NWO/FOM "Computational Sciences for Energy Research (CSER)" PhD program, project number 15CSER12

Cover design: Arrows depicting spin injection from a platinum to gold layer superimposed on the driving charge current distribution, stylized by Rohit Sasidharan Nair

Printed by: Gildeprint, Enschede, The Netherlands

ISBN: 978-90-365-5211-0

DOI: $10.3990 / 1.9789036552110$

(C)2021 Rohit Sasidharan Nair, The Netherlands. All rights reserved.

No parts of this thesis may be reproduced, stored in a retrieval system or transmitted in any form or by any means without permission of the author. Alle rechten voorbehouden. Niets uit deze uitgave mag worden vermenigvuldigd, in enige vorm of op enige wijze, zonder voorafgaande schriftelijke toestemming van de auteur. 


\title{
SPIN-ORBIT-COUPLING INDUCED LATERAL SPIN TRANSPORT FROM FIRST PRINCIPLES
}

\author{
DISSERTATION \\ to obtain \\ the degree of doctor at the University of Twente, \\ on the authority of the rector magnificus, \\ Prof. Dr. Ir. A. Veldkamp, \\ on account of the decision of the Doctorate Board \\ to be publicly defended \\ on Friday 27 August 2021 at 16:45 hours \\ by

\section{Rohit Sasidharan Nair} \\ born on 19 September, 1991 \\ in Tripunithura, India
}




\section{Composition of the Graduation Committee}

Prof. Dr. Jennifer L. Herek University of Twente, Chairman

Prof. Dr. Paul J. Kelly

University of Twente, Promotor

Dr. Mark D. Stiles

Prof. Dr. Yuriy Mokrousov

National Institute of Standards and Technology

Johannes Gutenberg-Universität Mainz

and Forschungszentrum Jülich

Prof. Dr. Bert Koopmans

Eindhoven University of Technology

Prof. Dr. Pepijn W.H. Pinkse

University of Twente

Prof. Dr. Ir. Hans Hilgenkamp University of Twente 


\section{Contents}

1 Introduction 1

1.1 Motivation . . . . . . . . . . . . . . . . 1

1.2 From spin to spintronics . . . . . . . . . . . . . . 1

1.3 Spin transport ................... 4

1.3.1 Spin flip diffusion . . . . . . . . . . . . 5

1.3.2 Spin Hall effect $\ldots \ldots \ldots \ldots$

1.3 .3 Interface effects $\ldots \ldots \ldots \ldots \ldots$

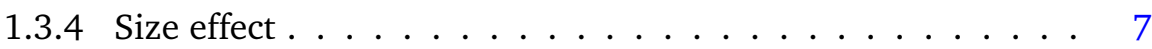

1.4 Method ....................... 7

1.4.1 Density functional theory $\ldots \ldots \ldots \ldots$

1.4 .2 Scattering theory . . . . . . . . . . . . 8

1.4 .3 Turning the heat up . . . . . . . . . . . . 8

1.4 .4 Computational scheme . . . . . . . . . . . . . . 10

1.5 Outline . . . . . . . . . . . . . . . . . 10

2 Spin transport in $5 d$ transition metals 13

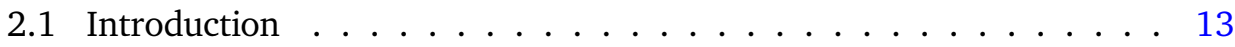

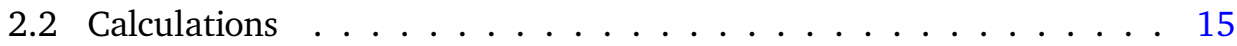

2.2.1 Transport calculations . . . . . . . . . . . . . . 15

2.2.2 Calculation of spin-orbit coupling parameter . . . . . . . 17

2.2.3 Calculation of electron mean free path, Fermi velocity and relaxation time . . . . . . . . . . . . 17

2.3 Results . . . . . . . . . . . . . . . . . . . . . 19

2.3 .1 Spin flip diffusion . . . . . . . . . . . . . . . 20

2.3 .2 Spin Hall effect . . . . . . . . . . . . . . . . . . . 25

2.3 .3 Anisotropy . . . . . . . . . . . . . . 33

2.4 Other calculations . . . . . . . . . . . . . . . . 33

$2.4 .1 \mathrm{Au} \ldots \ldots \ldots \ldots \ldots \ldots \ldots \ldots$

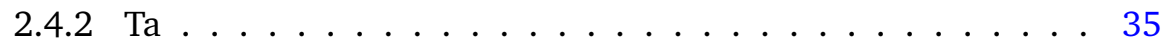

2.5 Summary and Conclusions . . . . . . . . . . . . . . 37 
3 Transverse spin currents in Fe-Ni systems 39

3.1 Introduction . . . . . . . . . . . . . . . . 39

3.2 Mott scattering . . . . . . . . . . . . . . . . . . . 42

3.3 Calculations . . . . . . . . . . . . . . 45

3.3.1 Magnetization parallel to the driving current . . . . . . . . 46

3.3.2 Magnetization perpendicular to the driving current . . . . . . 49

3.4 Discussion . . . . . . . . . . . . . . . . . 52

3.5 Appendices ........................ 53

3.5.1 Relationship between $\mathbf{s}$ and $\hat{\mathbf{m}} \ldots \ldots \ldots \ldots 53$

3.5.2 Mott analysis for $\mathbf{M} \perp j_{c}^{z} \ldots \ldots \ldots \ldots \ldots$

3.5.3 Comparison of CPA potentials . . . . . . . . . . 55

4 Fully resolved currents from quantum transport calculations $\quad 57$

4.1 Introduction . . . . . . . . . . . . . . . 57

4.2 Methods . . . . . . . . . . . . . . . . . 60

4.2 .1 Quantum transport . . . . . . . . . . . . . . . 60

4.2.2 Interpolation of interatomic currents onto a three dimensional grid ........................ 61

4.2 .3 Current streamlines . . . . . . . . . . . . . 65

4.2 .4 Mean Free Path . . . . . . . . . . . . . . . . . . . . 66

4.3 Results . . . . . . . . . . . . . . . . . . 67

4.3 .1 Knudsen limit . . . . . . . . . . . . . . . . . . . . 68

4.3 .2 Classical limit . . . . . . . . . . . . . . . . 72

4.3.3 Effect of different lead materials . . . . . . . . . . . . 77

4.4 Discussion . . . . . . . . . . . . . . . . . . . 79

5 Spin Hall Effect in a thin Pt film $\quad 81$

5.1 Introduction $\ldots \ldots \ldots \ldots \ldots \ldots$. . . . . . . 81

5.2 Method . . . . . . . . . . . . . . . 82

5.3 Calculations . . . . . . . . . . . . . 83

5.4 Improved Valet-Fert model . . . . . . . . . . . . . . . . . . 85

5.5 Surface enhancement . . . . . . . . . . . . . . . 88

5.6 Discussion . . . . . . . . . . . . . . . . . . 89

5.7 Summary . . . . . . . . . . . . . . . . . . 90

6 Transverse spin injection in bilayers $\quad 91$

6.1 Introduction . . . . . . . . . . . . . . . . . 91

6.2 Method ........................ . 92

6.3 Valet-Fert Model . . . . . . . . . . . . . . . . . . . 95

6.4 Results . . . . . . . . . . . . . . . . . . . . 97

$6.4 .1 \mathrm{Pt} \mid$ Au bilayer . . . . . . . . . . . . . . . 98

$6.4 .2 \mathrm{FM} \mid \mathrm{Cu}$ bilayers . . . . . . . . . . . . . . . 104 
6.4 .3 Comparison . . . . . . . . . . . . . . . . . 111

6.4 .4 Bulk spin polarization . . . . . . . . . . . . . 111

6.5 Conclusion . . . . . . . . . . . . . . . . . . . 113

$\begin{array}{ll}\text { A Mott scattering theory } & 115\end{array}$

$\begin{array}{ll}\text { Bibliography } & 123\end{array}$

$\begin{array}{ll}\text { Summary } & 139\end{array}$

$\begin{array}{ll}\text { Acknowledgements } & 141\end{array}$ 


\section{1 \\ Introduction}

"May I then conclude that the electron itself, spinning like a tiny gyroscope, is probably the ultimate magnetic particle"

A.H Compton 1921

\subsection{Motivation}

Everything digital is currently stored in data centres on magnetic hard disk drives which are accessed at will using a magnetic read head. The spinning disks and moving read heads in the hard disks imply slow access time and high power consumption making data centres giant sponges for electricity. At the same time, semiconductor based storage suffers from thermal dissipation with miniaturization on top of data leakage and high cost. The ideal memory for data storage would be cost-effective, non-volatile, fast and consume less power, combining desirable features of both magnetic and semiconductor storage technology. To this end, the field of "spintronics" promises a new direction towards efficient data storage using magnetic memories.

\subsection{From spin to spintronics}

The heuristic notion of an electron spinning like a top was proposed by Ralph de Laer Kronig in 1925 [2] in a meeting with Pauli in Tübingen and was immediately dismissed as "unphysical". A few months later, Uhlenbeck and Goudsmit would 


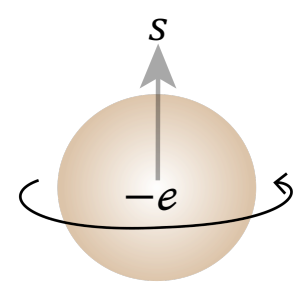

Figure 1.1: A picture of an electron imagined as a sphere of negative charge, $-e$, spinning about its axis giving rise to an angular momentum called spin, $\mathbf{s}$, along its axis of rotation and corresponding to this, a magnetic moment. Spin is an angular momentum measured in units of $\hbar$, magnetic moments are measured in units of the Bohr magneton $\mu_{B}$. When the direction of rotation is reversed, so is the spin.

hypothesise the same idea to interpret experiments on atomic spectra [3]. Many decades later this intrinsic angular momentum called spin has lent itself to nanotechnology in the modern pursuit of large storage capacity and the manipulation of data. The field of "spintronics" or spin-electronics is concerned with using the spin of electrons to store and manipulate data instead of, or in addition to, using the electron charge [4]; these are attractive for applications because they work at room temperature (RT).

A solid becomes magnetic when the spins of a majority of its electrons align in the same direction. A magnetic memory device can then be constructed by juxtaposing such magnetic domains; inside a domain, a majority of spins point in the same direction. A typical magnetic memory stores bits of information in the magnetic domains that point in 'up' and 'down' directions mimicking ' 1 's and ' 0 's. This information is then read from the boundaries between the domains using a magnetic read head whose fundamental building block is a spin valve [5, 6]. A spin-valve is simply a trilayer structure comprising two ferromagnetic (FM) layers sandwiching a paramagnetic/non-magnetic (NM) layer such that one of the ferromagnetic layers is a soft magnet whose magnetic moment can be easily tilted and the other a hard magnet whose moment is pinned. In a read-head, the soft layer is designed to have its magnetization perpendicular to the hard layer to maximize the sensitivity. When a read-head moves over the storage medium, the magnetic field emanating from the boundaries * between two domains can tilt the magnetic moment of the soft magnetic layer resulting in two configurations - one with a higher and the other with a lower electrical resistance leading to a high (1) and a low (0) voltage signal allowing information stored in the memory device to be read. This difference in resistance is referred to as Giant Magneto-Resistance(GMR) and

\footnotetext{
wall.

"The magnetic field is strongest at the boundary between two domains that is called a domain
} 
its observation in $1988[5,6]$ triggered the interest in spintronics for applications. The role of GMR has now been delegated to Tunnelling MagnetoResistance (TMR) $[7,8]$ which is an analogous change in resistivity for the tunnelling of a current signal across the spin valve configuration termed as a magnetic tunnel junction (MTJ) where the paramagnetic layer is replaced by a thin insulator. A gigantic TMR of over $600 \%$ has been demonstrated [9] making for an excellent read head mechanism and thus cementing the position of magnetic memories as the future of data storage.

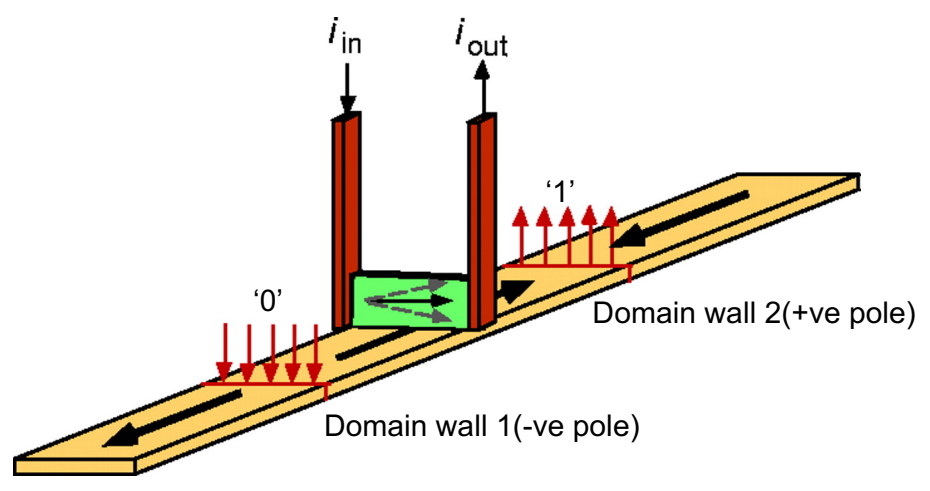

Figure 1.2: A schematic representation of a GMR read head (green) that passes over a storage medium (yellow). Bits of information are stored at the boundaries between two magnetic domains called domain walls where the magnetic field is strongest causing the magnetization of the soft magnetic layer of the GMR read head to rotate. The change in resistance and thereby the 'bits' can be sensed by passing a current $i$ through the read head. Adapted from Ref. [10]

Where is the catch? Writing data into a magnetic memory implies switching magnetic domains at will using torque exerted by an external source such as a spin transfer torque [11] by a spin polarized current that is usually prepared by passing a charge current through a ferromagnet. This poses a big scaling problem as miniaturization of magnetic memory invites larger current densities for writing which in turn leads to higher power consumption for smaller sizes and operational issues such as thermal instability due to Joule heating. How can we solve this? One of the promising ways to resolve the issue is to use a 'pure spin current ${ }^{\prime}$ to switch the domains(or bits) which reduces the power consumption and causes theoretically 'zero Joule heating' at the memory cells. A promising candidate to generate such

\footnotetext{
†A pure spin current is made up of 'up' and 'down' spins propagating in opposite directions resulting in a zero net charge current
} 
a pure spin current is the spin Hall effect [12] which will be introduced to the reader in the following section. Exploring the generation and transport of spins which are inextricable occupies the primary goals of spintronics. In this thesis, we study quantitative and qualitative aspects of spin transport in the context of its generation, detection, injection and miniaturization of a transport geometry.

\subsection{Spin transport}

The foundations for spin transport were laid in 1936 by Sir N.F.Mott [13]. His two-current model which considers a current flowing through a metal as being composed of two spin dependent conducting channels foreshadowed GMR in spin valves. Although intuitive and pedagogic, such simple models fail to capture microscopic details of electronic transport as related to highly complex Fermi surfaces of transition metals [14-16]. In order to make contact with modern experimental measurements, there is an urgent need for rigorous first-principles calculations that can accurately accommodate not only the electronic structure but also intrinsic temperature dependent disorder on an equal footing. Of the many effects of a spin, its coupling to the electron's orbital angular momentum known as spin-orbit coupling (SOC) is the key to many conspicuous properties in solids. The strength of the spin-orbit interaction energy is approximately proportional to the square of the number electrons in an atom [17], the heavier the atom, the bigger the coupling. As a consequence, SOC plays a significant role in electron and spin transport phenomena of transition metals. Spin flipping and spin Hall effect (SHE) schemat-

(a)

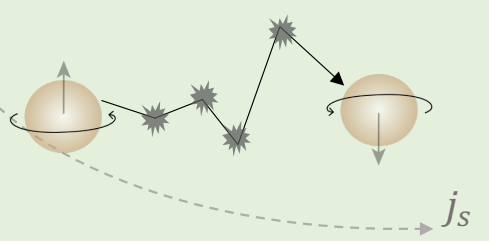

(b)

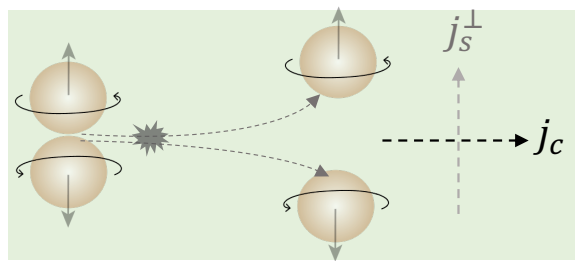

Figure 1.3: (a) A single spin undergoing multiple scattering constituting a random walk leading to a flip. The average distance between such spin flips is given by the spin flip diffusion length $l_{\mathrm{sf}}$. The dashed arrow in the background depicts the decay of an injected current of spins, $j_{s}$ caused by several spin flips as it passes through the material. (b) Asymmetric scattering of spins in a material leading to the separation of unlike spins in the lateral direction. The cumulative effect of such scattering events is called the spin Hall effect whereby a charge current $j_{c}$ injected into a material gives rise to a spin current $j_{s}^{\perp}$ in the transverse directions; the dimensionless conversion ratio $j_{s}^{\perp} / j_{c}=\Theta_{\mathrm{sH}}$ is the spin Hall angle. 
ically shown in Fig. 1.3 are two of the key spin-orbit coupling related properties. The magnitude of spin flipping and SHE are measured by the spin flip diffusion length $l_{\mathrm{sf}}$ and spin Hall angle $\Theta_{\mathrm{sH}}$ respectively.

\subsubsection{Spin flip diffusion}

As a spin traverses a material, it can change, for instance, an 'up' state can flip to 'down' and vice versa. A collective effect of such 'flips' is a net quenching or relaxation of a non-equillibrium spin current. The spin flip diffusion length $l_{\mathrm{sf}}$ is the length scale of spin flipping when a current of spins propagates through a material. At room temperature, the $l_{\mathrm{sf}}$ in a typical transition metal is $\sim 1-100 \mathrm{~nm}$ [18]. The accurate determination of $l_{\mathrm{sf}}$ has proven to be challenging in experiments as it is often sensitive to method of detection, sample preparation and extrinsic contributions such as interface effects. For instance, reported values of $l_{\text {sf }}$ for the most studied Pt span an order of magnitude [18-20]. Therefore, a theoretical benchmarking in pure samples is desirable to discern extraneous contributions to experimental measurements. According to a well-known Boltzmann transport model for spin diffusion derived by Valet and Fert [21], a non-equillibrium spin current injected at a boundary $(r=0)$ of a material decays exponentially as $\sim e^{-r / l s f}$ away from the boundary.

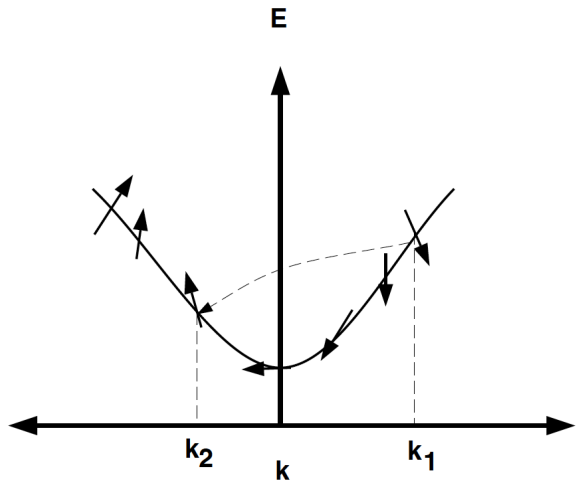

Figure 1.4: A parabolic energy band showing orientation of mixed spin states (arrow heads) at different $k$ points in reciprocal space. Scattering of an electron from $k_{1}$ to $k_{2}$ is accompanied by a change in its spin direction leading to relaxation. Adapted from Ref. [22]

There are several mechanisms by which spins can relax in a material such as the Elliott-Yafet [23, 24], Dyakanov-Perel [25], Bir-Aronov-Pikus [26] etc. Spin relaxation in metals at a finite temperature is generally attributed to the Elliott-Yafet mechanism. Elliott showed using simple free electron like bands that in the presence of SOC, the spin state of an electron is a wave-vector $k$ dependent quantity 
and is not purely 'up' or 'down' rather an admixture of the two. Thus any scattering from one wave-vector to another leads to a change in spin state and subsequently relaxation of spins as shown for a simple parabolic band in Fig. 1.4. The relationship with temperature was then worked out by Yafet for semiconductors a decade later. Very little work has been done to rigorously verify this in materials with complex Fermi surfaces to understand the role played by various electronic structure parameters such as density of states (DoS), bandwidth, lattice structure etc., in determining $l_{\mathrm{sf}}$ in a transition metal.

\subsubsection{Spin Hall effect}

Based upon relativistic treatment of electron scattering, Mott and Massey showed that 'up' and 'down' electrons will be scattered differently by an atom with SOC that leads to the spin polarization of an unpolarized electron beam [27]. This phenomenon was predicted to survive in paramagnetic solids [12, 25] and was experimentally observed for the first time little more than a decade ago [28, 29] so that a charge current passed through a solid with strong SOC excites pure spin currents in the transverse direction- the spin Hall effect(SHE). This is a reciprocal effect whereby a spin current injected into a paramagnetic solid will excite a charge current in the transverse direction called the inverse spin Hall effect (ISHE) that is often used in experiments to measure $\Theta_{\mathrm{sH}}$. Transverse spin currents generated by the SHE accumulate spins at the boundaries of a solid which can be injected into another material and is being studied with a view to switching the magnetization of an adjacent magnetic material and using this as a magnetic memory [30-33]. Because of their large SOC, only heavy paramagnetic magnetic metals like Pt group of metals $[34,35]$ were considered as suitable candidates for generating pure spin currents. However, recently it has been demonstrated that even light magnetic materials can be used to generate detectable transverse spin currents that are not oriented parallel to the magnetization [36-39], this has not yet been confirmed. In addition to the usual spin Hall effect, the symmetry breaking by magnetization can lead to other current components such as a spin swapping [40-42] and the anomalous Hall currents [43] that makes the physics more complicated and interesting in ferromagnets.

\subsubsection{Interface effects}

Interfaces between different materials are always encountered in spintronics applications and experiments. Theoretical studies [15, 44-47] have elucidated the crucial role played by interfaces in transport phenomena in the ballistic regime. In the diffuse regime, in which all experiments are performed, disentangling interfaces and bulk contributions is challenging without an exquisite knowledge of the details of the interface such as intermixing of atoms, grain boundaries and 
defects. Because an ideal interface is a two-dimensional boundary between two materials, ambiguity arises when defining the bulk properties needed to characterize transport through the interface. Nevertheless, it is useful to first introduce a third 'interface' material of finite thickness with bulk properties and then let its thickness vanish. In this limit, the interface resistivity $\rho_{\mathrm{I}}$ transforms into an areal resistance $A R_{\mathrm{I}}$ [48], $l_{\mathrm{sf}}$ manifests itself as a spin memory loss parameter $\delta[19,48]$ and the spin Hall effect is given by an interface spin Hall angle $\Theta_{\mathrm{I}}$ [34]. In addition, the need to be careful about a possible anisotropy in the interface quantities to distinguish in-plane and out of plane transport makes the analysis complicated.

\subsubsection{Size effect}

One of the biggest concerns facing the nano-electronics community is the increase in electrical resisitivity accompanying miniaturization of circuits. This size effect is a consequence of the emergence of scattering contributions from grain boundaries and surfaces when the thickness (radius) of a metallic film (wire) is reduced to the order of the electron mean free path $\lambda$ of the material [49]. The mean free path $\lambda$ is determined by the electronic structure of the material and is a function of temperature in pure metals. Even today, this size effect is almost universally addressed using semi-classical models [50,51] developed some 50-60 years ago with the goal of determining an average resisitivity of the geometry. However, in the field of spintronics, one is concerned with microscopic details of transport through multilayer geometries where the constituent layers are usually of 10-100 nanometres thicknesses. There is an urgent need for a direct method to study current flow in SHE experiments [52, 53]

\subsection{Method}

Here we outline our computational approach used to obtain all the quantitative results presented in this thesis.

\subsubsection{Density functional theory}

The first step towards studying electron transport in a material is calculating the electronic structure of the material. For an interacting $\mathrm{N}$ electron system it translates to solving a wavefunction in a $3 \mathrm{~N}$ dimensional space. This computationally unrealisable task is circumvented by density functional theory (DFT) which introduced the electron density that minimizes the total energy of the system as the basic ground state property [54]. Kohn and Sham [55] showed that the "effect" of electron-electron interactions can be described by a local potential that is a functional of the electron density. Using the local effective potential, the coupled $\mathrm{N}$ 
electron wave equations can be replaced by N Schrödinger-like independent electron equations. Solving the independent electron equation and another equation that expresses electron density in terms of the wave function, together known as the Kohn-Sham (KS) equations iteratively to self-consistency, the ground state of the system can be obtained. The combination of local spin density approximation (LSDA) and density functional theory provides an accurate description of the electron wave functions that allows us to handle transport in complex materials entirely from first-principles.

\subsubsection{Scattering theory}

The quantum transport calculations presented in this thesis are based upon a scattering theory [56] applied to a two terminal geometry as shown Fig. 1.5. The two terminal geometry is constructed by sandwiching the disordered material referred to as the scattering region $\mathcal{S}$ sandwiched between lattice matched ballistic leads on the left $\mathcal{L}$ and on the right $\mathcal{R}$. The scattering theory is formulated in terms of a scattering matrix $\mathbf{S}$ that consists of $t$ and $r$ that are themselves matrices of transmission $t$ and reflection $r$ probability amplitudes respectively that relate how an incoming state $I\left(I^{\prime}\right)$ from a lead is scattered forward or backward by disorder into an outgoing state $O\left(O^{\prime}\right)$ as,

$$
\left(\begin{array}{c}
O \\
O^{\prime}
\end{array}\right)=\underbrace{\left(\begin{array}{ll}
r & t^{\prime} \\
t & r^{\prime}
\end{array}\right)}_{\mathbf{S}}\left(\begin{array}{c}
I \\
I^{\prime}
\end{array}\right)
$$

In order to obtain the scattering matrix, we make use of a generalization [57] by Ando [56] which is based upon matching the wave function (WFM) of the scattering region to the Bloch modes of the leads which are ballistic and assumed to be semi-infinite. The scattering matrix is a useful tool, for e.g. using the LandauerBüttiker formalism [58,59], conductance of the scattering region $\mathcal{S}$ can be directly estimated from the scattering matrix as $\sim \operatorname{Tr}\left(t t^{\prime}\right)$ in the linear response regime.

\subsubsection{Turning the heat up}

At a finite temperature $T>0 \mathrm{~K}$, the kinetic energy of atoms manifests itself in their displacement from the equilibrium lattice positions. This 'intrinsic' lattice disorder is what predominantly gives a material its temperature dependent resistivity [60]. To keep the spirit of a true "ab-initio" approach, we could in principle calculate the phonon spectrum of the scattering material and populate the phonon modes corresponding to the given temperature and thereby introduce disorder in the system [61]. However, given the wealth of experimentally determined temperature dependent resistivities [62], we shall adopt the following approach. We include the lattice disorder in our scattering region by displacing atoms using an 
uncorrelated Gaussian distribution of displacements constructed using a root-mean squared displacement $\Delta(T)_{r}$ chosen to reproduce the experimental resistivity at a given temperature $T$. In a magnetic material, in addition to the lattice disorder, the atomic moments are randomly tilted away from their mean positions reducing the average magnetic moment as a function of temperature ultimately leading to a paramagnetic state above the Curie temperature [60]. Analogues to the implementation of lattice disorder, a Gaussian distribution of root mean square rotation $\Delta(T)_{\theta}$ of the polar angle of the atomic spins is chosen to reproduce the experimental bulk magnetization at a given temperature $T$ such that $\Delta(T)_{r}$ and $\Delta(T)_{\theta}$ together reproduces the experimental resistivity $\rho(T)$ of the magnetic material [57].

We include this disorder in our scattering region by taking advantage of the adiabatic approximation [63] which states that in the conduction electron frame of reference, the atomic potentials can be treated as static because the electron momentum relaxation time $\left(10^{-14}-10^{-15} \mathrm{~s}\right)$ is much shorter than the atomic vibration period $\left(\sim 10^{-12} \mathrm{~s}\right)$. We construct several random configurations of atomic disorder and perform a calculation for each such static configuration; the final results presented in this thesis are averaged over a number of such configurations.

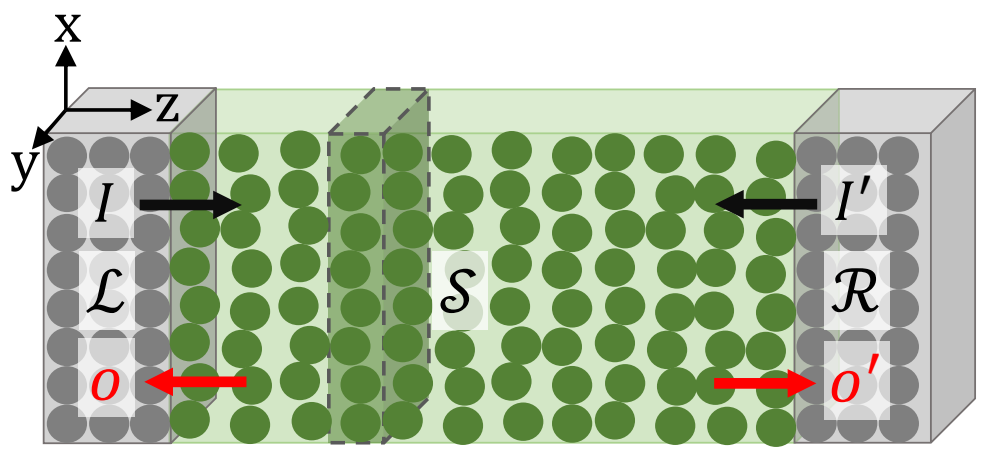

Figure 1.5: Schematic of a two terminal geometry constructed by sandwiching a configuration of a scattering region $\mathcal{S}$ between lattice matched leads on the left $(\mathcal{L})$ and right $(\mathcal{R})$. Atoms in the scattering region are randomly displaced from their mean position by temperature. In the scattering region, a supercell (e.g. the dashed box) at a given value of $z$ is repeated periodically in $x$ and $y$ directions. The incoming states from $\mathcal{L}(\mathcal{R}), I\left(I^{\prime}\right)$ and the outgoing states $O\left(O^{\prime}\right)$ are schematically depicted as black and red arrows respectively. Cartesian coordinate axes are shown for reference 


\subsubsection{Computational scheme}

Our calculations proceed in two steps. We first calculate the charge and spin densities, the Kohn-Sham potentials and the ground state Fermi energy of the materials constituting the leads and scattering region. We do so, by using a tight binding code based upon linearized muffin tin orbitals (LMTO) and the atomic sphere approximation (ASA) $[64,65]$. In the next step, we apply an infinitesimal voltage across the terminals (leads) and calculate the wave function of the perturbed system in the so called linear response regime. The wave function is determined by the aforementioned scattering formalism. An efficient implementation of the scattering scheme using minimal tight binding linearized muffin tin orbitals (TB-LMTO) $[66,67]$ combined with local spin density approximation (LSDA) within the framework of density functional theory (DFT)have been encoded over the years in the Computational Materials Science group at the University of Twente. In applying the scheme, we solve the single-particle Schrödinger equation to obtain eigen functions of the disordered scattering region. Disorder in the system breaks translational symmetry and makes solving the Schrödinger equation impossible. To circumvent this we introduce periodic boundary conditions in the $x y$ plane by constructing finite size $N \times M$ 'lateral supercells' where $N$ and $M$ are the number of atoms in the $x$ and $y$ directions respectively such that the disordered supercell is repeated periodically (see Fig. 1.5). Taking advantage of Bloch's theorem [60] in the $x$ and $y$ directions, we can now solve the single particle Schrödinger equation for the scattering region using Bloch modes in the ballistic leads as boundary conditions in the $z$ direction. Our scattering formalism yields the coefficients that describe the scattering states throughout the scattering region allowing us to calculate the twelve-vector $\sim \boldsymbol{v} \otimes\{e, \sigma\}$ that describes currents of charge and spin everywhere in the scattering region [20]. The local velocities can be calculated using the equation of motion $\boldsymbol{v}=d \boldsymbol{r} / d t=[H, r]$. Calculating local currents allows us to make contact with the diffusion theories of spin transport $[21,68]$ which are universally used in interpreting experiments and thereby extract transport parameters of interest. We follow this approach in the remaining chapters of this thesis.

\subsection{Outline}

The impetus for the research that has gone into realizing this thesis was fuelled by uncertainties in the reported spin transport parameters [18-20] and the gap between phenomenological models and experiments in (spin)transport phenomena. In this thesis, we aim to resolve some of the uncertainties by pushing our computational capabilities in investigating microscopic details of transport. Furthermore, we attempt to improve upon existing models of interpretation/prediction used in experiments and application.

In Chapter 2, we establish quantitative benchmarks for the two most impor- 
tant parameters $l_{\mathrm{sf}}$ and $\Theta_{\mathrm{sH}}$ used to describe spin transport in $5 d$ transition metal series as a function of temperature. In doing so, we discover the interplay between the lattice, electronic structure and disorder in determining interesting trends across the series. We identify key properties of metals that could be tuned to control spin transport in heavy metals.

In Chapter 3, we investigate the generation of spin currents in the light $3 \mathrm{~d}$ ferromagnets Fe-Ni systems and apply Mott scattering analysis to trace the origin of various spin current components. We estimate the values for spin Hall angle and the recently demonstrated spin swapping (SW) angles as a function of alloying at room temperature. We further explore the dependence of these currents on the direction of magnetization of the ferromagnets.

In Chapter 4, we extend the planar averaged current scheme [20] to a fully resolved transport scheme which would allow us to study local transport quantities in all the three directions, $x, y, z$ at atomic resolution! We demonstrate the scheme by studying charge transport in thin films of $\mathrm{Pt}$ and $\mathrm{Au}$ as well as bilayers $\mathrm{Pt} \mid \mathrm{Au}$ and Pt|Ir. We study transport in 'classical' and 'Knudsen' limits determined by the sample thickness relative to the mean free path $\lambda$ of the materials and explicitly illustrate how charge currents streamline in nanoscale geometries.

In Chapter 5, using the fully resolved transport scheme developed in the previous chapter we calculate the spin Hall effect in a thin film of Pt at room temperature. We reveal the failure of existing diffusion model in sufficiently describing the transport and accumulation of intrinsically generated spin currents and present an improved model by taking into account of the role played by the surface.

In Chapter 6, we address the question:- How efficiently can we inject a pure spin current generated in one material by spin Hall effect into another? We do so by combining a number of elements developed in the thesis up to this point. An explicit SHE experiment is conducted on the computer by driving a charge current through a CIP geometry constructed using bulk-like non magnetic Pt and ferromagnetic $\mathrm{Py}(\mathrm{Ni})$ slabs as spin generators and $\mathrm{Au}$ and $\mathrm{Cu}$ slabs as spin sinks respectively . Using the fully resolved current scheme, we quantify the injection efficiencies from $\mathrm{Pt}$ and $\mathrm{Py}(\mathrm{Ni})$ into $\mathrm{Au}$ and $\mathrm{Cu}$ respectively. By extending the Valet-Fert model of spin diffusion to include SHE and interface effects, we extract parameters like spin memory loss $\delta$ and interface spin Hall effect $\Theta_{\mathrm{I}}$ for the paramagnetic Pt|Au bilayer. 


\section{2}

\section{Spin-transport parameters in $\mathbf{5 d}$ transition metals}

The spin-flip diffusion length $l_{\mathrm{sf}}$ and spin Hall angle $\Theta_{\mathrm{sH}}$ are key transport parameters in the field of spintronics whose origin is the relativistic spin-orbit coupling. We use a first-principles density-functional-theory based implementation of scattering theory and realistic temperature-induced lattice disorder to perform a comprehensive study of $l_{\mathrm{sf}}$ and $\Theta_{\mathrm{sH}}$ for all $5 d$ transition metals. We illustrate how the factors band-filling and crystal structure that are not considered in phenomenological models determine trends across the series. $l_{\mathrm{sf}}$ does not decrease monotonically with the atomic number $Z$ but, as a function of the number of $5 \mathrm{~d}$ electrons, is found to be inversely proportional to the density of states at the Fermi level. $\Theta_{\mathrm{sH}}$ is qualitatively driven by the density of states while degeneracies at points of high symmetry in the Brillouin zone play a pivotal role in determining trends. We show that the products $\rho(T) l_{\mathrm{sf}}(T)$ and $\Theta_{\mathrm{sH}}(T) l_{\mathrm{sf}}(T)$ are constant for experimentally relevant temperatures.

\subsection{Introduction}

Spin-orbit coupling (SOC) leads to the loss of spin angular momentum. A current of electrons injected from a ferromagnet into a nonmagnetic material loses its spinpolarization over a length scale of $l_{\mathrm{sf}}$, the spin-flip diffusion length (SDL) [21, 69-

"A short version of this chapter has been published as: R. S. Nair, E. Barati, K. Gupta, Z. Yuan, and P. J. Kelly, "Spin-Flip Diffusion Length in $5 d$ Transition Metal Elements: A First-Principles Benchmark", Phys. Rev. Lett. 126, 196601 (2021). 
72], making the observation of spin currents difficult. The giant magnetoresistance (GMR) effect was discovered in magnetic multilayers $[5,6]$ only when the thickness of the spacer layers separating the magnetic films was made to be of order $l_{\mathrm{sf}}$. A review of the SDL in metals and alloys some twenty years after the discovery of GMR concerned mainly the free-electron like metals $\mathrm{Cu}, \mathrm{Ag}$, $\mathrm{Au}$ and $\mathrm{Al}$ that have large values of $l_{\mathrm{sf}}$; for just a few of the transition metal elements, there was a single, low temperature entry [18]. With the "rediscovery" $[12,68]$ of the spin Hall effect (SHE) [25, 73] and its observation in semiconductors [28, 29] and metals $[74,75]$, this situation has changed radically [76, 77]. However, even for wellstudied materials like Pt, values of $l_{\mathrm{sf}}$ reported over the last decade span an order of magnitude $[20,77]$. Discernible trends in $l_{\mathrm{sf}}$ have not been reported for different transition metal elements.

The SHE is another consequence of SOC whereby the passage of a charge current through a metal gives rise to a transverse spin current that can enter an adjacent magnetic material and exert a torque on its magnetization causing it to switch its orientation. The efficiency of the SHE is given by the spin Hall angle (SHA) $\Theta_{\mathrm{sH}}$ that is the ratio of the transverse spin current (measured in units of $\hbar / 2$ ) to the charge current (measured in units of the electron charge $-e$ ). From being a curiosity, the SHE has rapidly become a leading contender to form the basis for a new magnetic memory technology [78] bringing with it the need to find materials with optimal values of $\Theta_{\mathrm{sH}}$ with a primary focus on heavy metals like Pt $[75,79]$, Ta [30] and W [80]. Striking discrepancies between different room temperature (RT) measurements, with reported values of e.g. $\Theta_{\mathrm{sH}}^{\mathrm{Pt}}$ ranging between $1 \%$ and $11 \%$ [76], led to the realization that the bulk parameters $l_{\mathrm{sf}}$ and $\Theta_{\mathrm{sH}}$ as well as the resistivity $\rho$ were very sample dependent and needed to be determined simultaneously. Doing this did not however lead to a consensus about the values of these parameters [77]. Whether the SHA is determined using spin pumping and the inverse SHE [75, 81-83], the SHE and spin-transfer torque [84], or nonlocal spin-injection $[79,85]$, interfaces are always involved leading to the suggestion that interface processes like interface spin flipping (spin memory loss) [19, 86-88] or an interface SHE $[34,89]$ should be taken into consideration in interpreting experiment. Attempts to do so have, if anything, made matters worse with recently determined values of $l_{\mathrm{sf}}^{\mathrm{Pt}}$ ranging from 1.4 to $11 \mathrm{~nm}$ and $\Theta_{\mathrm{sH}}^{\mathrm{Pt}}$ ranging between $3 \%$ and 39\%; see Table V in Ref. [20]. The only attempt we are aware of to study the intrinsic SDL theoretically is the phonon-induced spin relaxation work by Fabian and Das Sarma on aluminium [90] that is not readily generalized to transition metals. Fig. 2.1 shows the experimental resistivity for all $5 d$ transition metals in a range relevant for experiments where the resistivity is dominated by electronphonon scattering. As we move along the series from $\mathrm{Hf}$ to $\mathrm{Pt}$, the resistivity varies in a non-trivial fashion. In this chapter we are interested in addressing how $\Theta_{\mathrm{sH}}$ and $1_{\mathrm{sf}}$ behave along the series and what determines their trends. A brief account of this work was already given in Ref. [93]. 


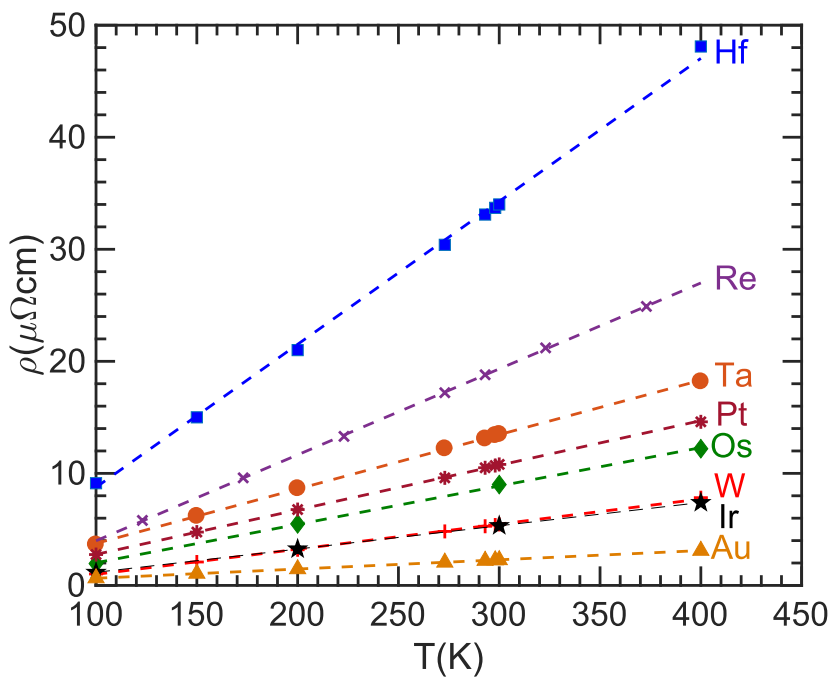

Figure 2.1: Experimental resistivities of $5 d$ metals as a function of temperature $T$ $[62,91,92]$. Dotted lines are a guide for the eye.

This chapter is organised as follows: we begin in Sec. 2.2 by briefly describing the scattering formalism we use to calculate $l_{\mathrm{sH}}$ and $\Theta_{\mathrm{sH}}$. We use a semi-classical approach to determine other important transport parameters such as the mean free path, Fermi velocity and relaxation time. Calculated results are presented Sec. 2.3 and a detailed analysis on the quantitative as well as qualitative effect of various parameters on spin flip diffusion and spin Hall effect. Effect of computational parameters on our calculated values are illustrated with examples in Sec. 2.4. A summary and some important conclusions are drawn in Sec. 2.5.

\subsection{Calculations}

\subsubsection{Transport calculations}

All calculations are performed within the framework of density functional theory (DFT) with local spin density approximation(LSDA) for exchange correlation energy and potentials. We solve the quantum mechanical scattering problem for a two terminal geometry $\mathcal{L}|\mathcal{S}| \mathcal{R}$ as illustrated in Fig. 2.2. The wave function of scattering region $\mathcal{S}$ is matched to the Bloch modes of the ballistic left $\mathcal{L}$ and right $\mathcal{R}$ lead using a wave-function matching (WFM) scheme[56]. We use a generalized tight-binding (TB) implementation of the scheme[16, 94] based upon linearized muffin-tin orbitals (LMTO) $[95,96]$ by including SOC and non-collinearity. Our scattering calculations include temperature induced lattice and spin disorder which breaks the 


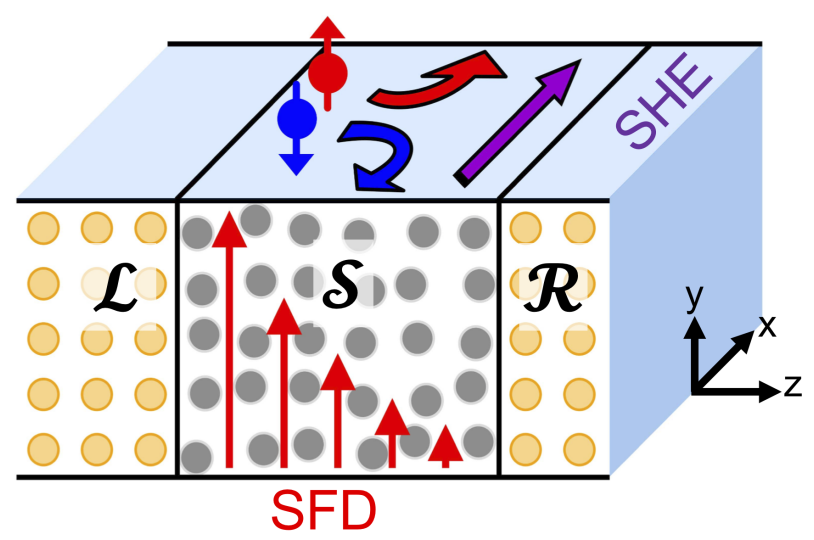

Figure 2.2: Schematic diagram of a disordered configuration of scattering geometry $\mathcal{S}$ sandwiched between semi-infinite $\operatorname{left}(\mathcal{L})$ and $\operatorname{right}(\mathcal{R})$ leads. For performing calculations at real temperature, several such configurations are generated by introducing temperature dependent displacements of atoms of $\mathcal{S}$ in a Gaussian manner. A fully polarized spin current injected from the left lead $\mathcal{L}$ undergoes spin flipping leading to spin equilibration on a length scale given by the spin-flip diffusion (SFD) length $l_{\mathrm{sf}}$ as suggested by the red arrows. An unpolarized charge current injected from $\mathcal{L}$ undergoes spin dependent scattering leading to a transverse spin Hall current given by the spin Hall angle $\Theta_{\mathrm{sH}}$ depicted by the purple arrow.

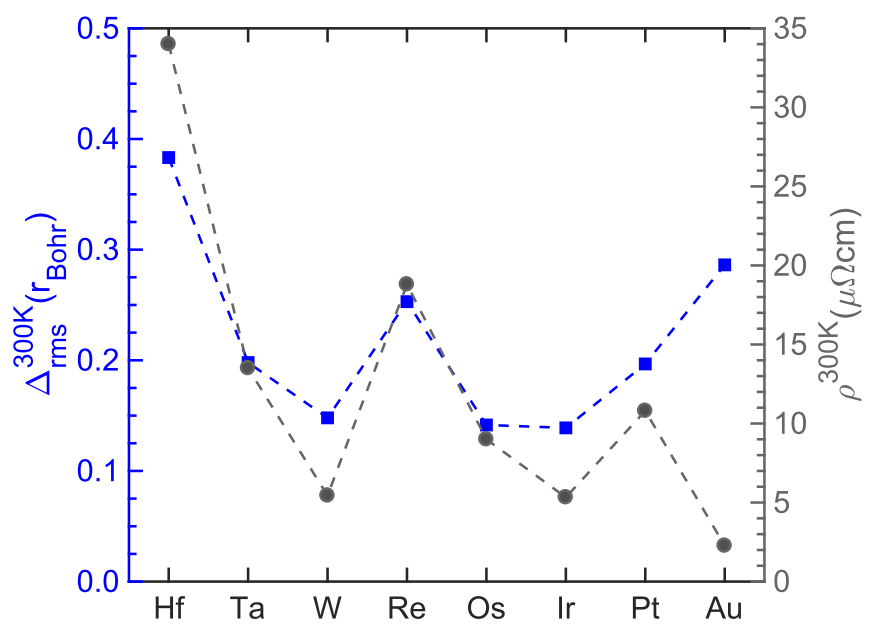

Figure 2.3: The root-mean-square displacements $\Delta_{\mathrm{rms}}$ used to generate Gaussian lattice disorder at $T=300 \mathrm{~K}$ for all $5 \mathrm{~d}$ transition metals. The corresponding resistivity values $\rho^{300 \mathrm{~K}}$ are plotted for reference. 
translational symmetry within the scattering region. In order to solve the KohnSham(KS) equations for the scattering region, we construct lateral "supercells" of finite size $N \times M$ that are assumed to be periodic in the $x y$ plane. From the solutions of the scattering calculations, we evaluate local charge and spin currents [20] to study the bulk transport properties SFD and SHE that are schematically sketched in Fig. 2.2.

To simulate temperature induced lattice disorder, we make use of the adiabatic approximation which allows us to treat the atoms as being stationary with respect to the itinerant electrons. Atoms are displaced using a Gaussian distribution of atomic displacements constructed using a root mean square displacement $\Delta_{\mathrm{rms}}$ to construct a disordered configuration. The $\Delta_{\mathrm{rms}}$ is determined to reproduce the experimental resistivity $\rho(T)$ corresponding to the temperature $T$ of interest, see Fig. 2.3. The final results presented are obtained by averaging over several such configurations. From now on, we will drop the subscript (rms) for simplicity.

\subsubsection{Calculation of spin-orbit coupling parameter}

Here we present how spin-orbit coupling is included in the transport calculations. Spin orbit coupling is introduced in the Hamiltonian using the Pauli term [57, 64],

$$
\begin{aligned}
\mathcal{H}_{\text {SO }} & =\frac{1}{c^{2} r} \frac{d V(r)}{d r} \mathbf{l} . \mathbf{s} \\
& =\xi\left(\varepsilon_{v}\right) \mathbf{l} . \mathbf{s}
\end{aligned}
$$

where $\xi\left(\varepsilon_{v}\right)$ is the spin-orbit coupling parameter calculated at an energy $\varepsilon_{v}$ in the band structure of the material. The spin-orbit coupling parameter for outer $d$ orbitals is given by

$$
\xi_{l}\left(\varepsilon_{v}\right)=\lambda_{\mathrm{sc}} \int \frac{1}{c^{2} r} \frac{d V(r)}{d r} \phi_{l}\left(\varepsilon_{v}, r\right)^{2} d r
$$

where $\phi_{1}(\varepsilon, \mathrm{r})$ is a partial wave obtained by solving the radial Schrödinger equation for energy $\varepsilon_{v}$ and angular momentum $l$ for the $d$ orbitals and $\lambda_{\mathrm{sc}}$ is an artificial factor (unity by default) that can be varied to study the effect of scaling the spin orbit coupling on transport parameters.

\subsubsection{Calculation of electron mean free path, Fermi velocity and re- laxation time}

The electrical conductivity in the relaxation time approximation for a multi-band real metal is given by[60]

$$
\sigma_{i j}=\sum_{\mu} \sigma_{i j}^{\mu}=\sum_{\mu} e^{2} \tau \int \frac{d^{3} k}{8 \pi^{3}} v_{i}^{\mu}(\mathbf{k}) v_{j}^{\mu}(\mathbf{k})\left(-\frac{\partial f}{\partial \varepsilon}\right)_{\varepsilon=\varepsilon_{\mu}(\mathbf{k})} .
$$


Here $\boldsymbol{v}^{\mu}$ is the electron velocity of band $\mu, f$ is the Fermi-Dirac distribution function and $\mathbf{k}$ is an arbitrary vector in the Brillouin zone. We first replace the derivative of the distribution function by a $\delta$-function at the Fermi energy,

$$
\begin{aligned}
\left(-\frac{\partial f}{\partial \varepsilon}\right)_{\varepsilon=\varepsilon_{\mu}(\mathbf{k})} & =\int d \varepsilon\left(-\frac{\partial f}{\partial \varepsilon}\right) \delta\left(\varepsilon-\varepsilon_{\mu}(\mathbf{k})\right) \\
& =\quad \delta\left[\varepsilon_{F}-\varepsilon_{\mu}(\mathbf{k})\right]
\end{aligned}
$$

Substituting the above $\delta$ function into (2.3) we find,

$$
\sigma_{i j}=\sum_{\mu} e^{2} \tau \int \frac{d^{3} k}{8 \pi^{3}} v_{i}^{\mu}(\mathbf{k}) v_{j}^{\mu}(\mathbf{k}) \delta\left[\varepsilon_{F}-\varepsilon_{\mu}(\mathbf{k})\right]
$$

where $\sum_{\mu} \int \frac{d^{3} k}{8 \pi^{3}} \delta\left[\varepsilon_{F}-\varepsilon_{\mu}(\mathbf{k})\right]=g\left(\varepsilon_{F}\right)$, the density of states at the Fermi energy. In a free-electron model, $\varepsilon(\mathbf{k})=\hbar^{2} k^{2} / 2 m$ and the Fermi velocity $\boldsymbol{v}_{F}=\hbar \mathbf{k}_{F} / m$.

$$
\begin{aligned}
& \varepsilon(\mathbf{k})=\frac{\hbar^{2} k^{2}}{2 m}, \\
& k_{x}^{2}\left(\varepsilon_{F}\right)+k_{y}^{2}\left(\varepsilon_{F}\right)+k_{z}^{2}\left(\varepsilon_{F}\right)=k_{F}^{2}, \\
& \boldsymbol{v}=\frac{\hbar \mathbf{k}}{m}, \\
& g\left(\varepsilon_{F}\right)=\frac{m k_{F}}{\pi^{2} \hbar^{2}}=\frac{3 n}{2 \varepsilon_{F}},
\end{aligned}
$$

where $n$ is the electron density. Here we are able to reproduce the Drude conductivity,

$$
\begin{aligned}
\frac{1}{3} \operatorname{Tr} \tilde{\sigma}= & \frac{\sigma_{x x}+\sigma_{y y}+\sigma_{z z}}{3} \\
& =e^{2} \tau \frac{3 n}{2 \varepsilon_{F}} \frac{\hbar^{2} k_{F}^{2}}{3 m^{2}}=\frac{n e^{2} \tau}{m}
\end{aligned}
$$

Alternatively, we can represent the conductivity in terms of the diffusion constant $D$,

$$
\frac{1}{3} \operatorname{Tr} \tilde{\boldsymbol{\sigma}}=e^{2} \tau g\left(\varepsilon_{F}\right) \frac{\hbar^{2} k_{F}^{2}}{3 m^{2}} \equiv e^{2} g\left(\varepsilon_{F}\right) D
$$

such that

$$
D=\tau \frac{\hbar^{2} k_{F}^{2}}{3 m^{2}}=\frac{v_{F}^{2} \tau}{3}
$$

If we define the mean free path $\lambda \equiv v_{F} \tau$, we are able to calculate from

$$
\lambda=\frac{3 D}{v_{F}}
$$


At an arbitrary $k$ point in a Brillouin zone, we can find the energy dispersion for a real metal $\varepsilon_{\mu}(\mathbf{k})$ and the corresponding electron velocity for a specific $\mathbf{k}$ and band $\mu$

$$
\boldsymbol{v}_{\mu}(\mathbf{k})=\frac{1}{\hbar} \frac{\partial \varepsilon_{\mu}(\mathbf{k})}{\partial \mathbf{k}}
$$

We define a Fermi velocity by averaging the electron velocity over the Fermi surface

$$
\begin{aligned}
\left\langle\boldsymbol{v}_{F}^{2}\right\rangle & =\frac{\sum_{\mu} \int \frac{d^{3} k}{8 \pi^{3}}\left\{\left[v_{x}^{\mu}(\mathbf{k})\right]^{2}+\left[v_{y}^{\mu}(\mathbf{k})\right]^{2}+\left[v_{z}^{\mu}(\mathbf{k})\right]^{2}\right\} \delta\left[\varepsilon-\varepsilon_{\mu}(\mathbf{k})\right]}{\sum_{\mu} \int \frac{d^{3} k}{8 \pi^{3}} \delta\left[\varepsilon-\varepsilon_{\mu}(\mathbf{k})\right]} \\
& =\frac{1}{g\left(\varepsilon_{F}\right)} \sum_{\mu} \int \frac{d^{3} k}{8 \pi^{3}}\left\{\left[v_{x}^{\mu}(\mathbf{k})\right]^{2}+\left[v_{y}^{\mu}(\mathbf{k})\right]^{2}+\left[v_{z}^{\mu}(\mathbf{k})\right]^{2}\right\} \delta\left[\varepsilon-\varepsilon_{\mu}(\mathbf{k})\right]
\end{aligned}
$$

Note that (2.11) yields an average conductivity for an isotropic medium. For uniaxial crystals such as hcp metals, we have anisotropic diffusive transport in directions parallel and perpendicular to the hexagonal axis. Consequently, the conductivity can be written as a tensor,

$$
\sigma=e^{2} g\left(\varepsilon_{F}\right)\left(\begin{array}{ccc}
D_{\perp} & 0 & 0 \\
0 & D_{\perp} & 0 \\
0 & 0 & D_{\|}
\end{array}\right)
$$

Without loss of generality we can write [97]

$$
D_{i}=\frac{1}{3} v_{i} \lambda_{i}=\frac{1}{3} v_{i}^{2} \tau_{i}, \quad i=\|, \perp
$$

To simplify the above we assume an average velocity $v_{F} \equiv\left\langle v_{F}^{2}\right\rangle^{\frac{1}{2}}$ and attribute the anisotropy to $\tau$ and $\lambda$. The spin-flip diffusion length $l_{\mathrm{sf}}$ and the spin relaxation time $\tau_{\mathrm{sf}}$ are related as $l_{\mathrm{sf}}^{2}=D \tau_{\mathrm{sf}}$ using which we can determine $\tau_{\mathrm{sf}}$. The ratio of the spin relaxation time, $\tau_{\text {sf }}$, to $\tau$ is finally

$$
\frac{\tau_{\mathrm{sf}}}{\tau}=\left[e^{2} \rho l_{\mathrm{sf}} g\left(\varepsilon_{F}\right)\right]^{2}\left\langle v_{F}^{2}\right\rangle .
$$

\subsection{Results}

Using the first principles method described in the previous section we determine $l_{\mathrm{sf}}$ and $\Theta_{\mathrm{sH}}$ for hcp Hf, Re and Os, bcc Ta and W and fcc Ir, Pt and Au as a function of temperature. The role of temperature, SOC, bandfilling and lattice structure in determining the trends across the series will be discussed in some detail in the following. 


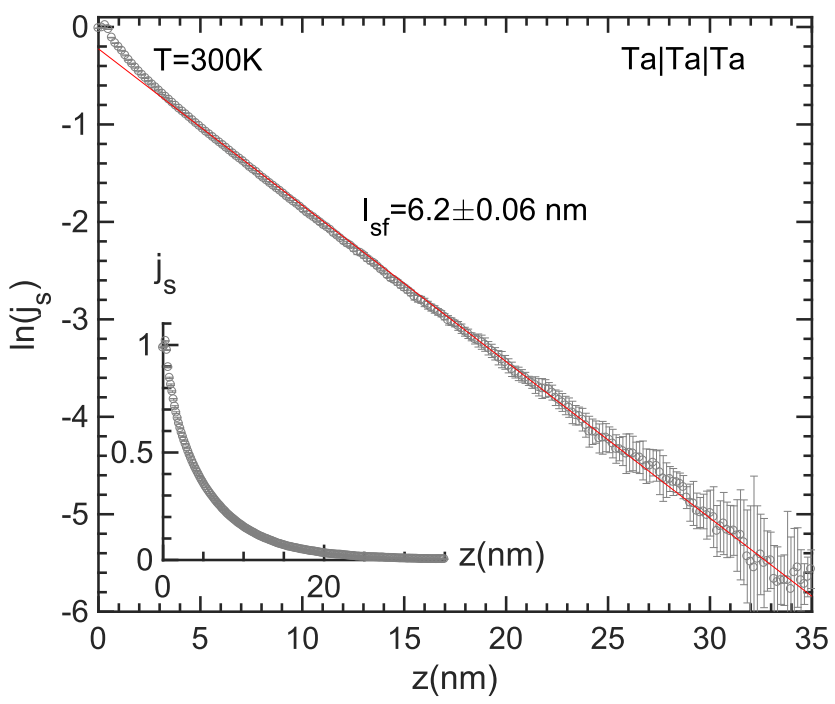

Figure 2.4: Natural logarithm of the spin current injected at 300K from a fully polarized left Ta lead into the scattering region as a function of the $z$ coordinate. The absolute value of the inverse slope of the fitting curve (red) gives $l_{\mathrm{sf}}$. The inset shows the decay of the spin current on a linear scale. This calculation was carried out for a $7 \times 7$ supercell with a BZ sampling of $23 \times 23 \mathbf{k}$ points. Error bars represent the mean deviation over 10 configurations of random disorder.

\subsubsection{Spin flip diffusion}

To calculate the $l_{\text {sf }}$ of the bulk material, we inject a fully polarised spin current from a lead into the scattering material. This is achieved by constructing half metallic ferromagnetic leads [20]. $l_{\text {sf }}$ is extracted using the Valet-Fert theory of spin relaxation [21] which describes the exponential decay of a spin current in the transport direction $z$. By plotting the natural log of the local spin current density as function of $z, l_{\mathrm{sf}}$ can be extracted as the inverse of the slope. Mathematically,

$$
j_{s}(z)=C e^{-z / 1_{\mathrm{sf}}}
$$

where $j_{s}(z)$ is the spin current density normalized to the charge current in the transport direction for a material with spin flip diffusion length $l_{\mathrm{sf}}$. In Fig. 2.4 we show an example of Ta at 300K. The "interface" effect between ballistic and diffusive Ta is apparent near the left lead $(z=0)$. More noise near the right lead is a result of the reduction in the number of states as a function of the distance from the left lead because of spin flipping. 


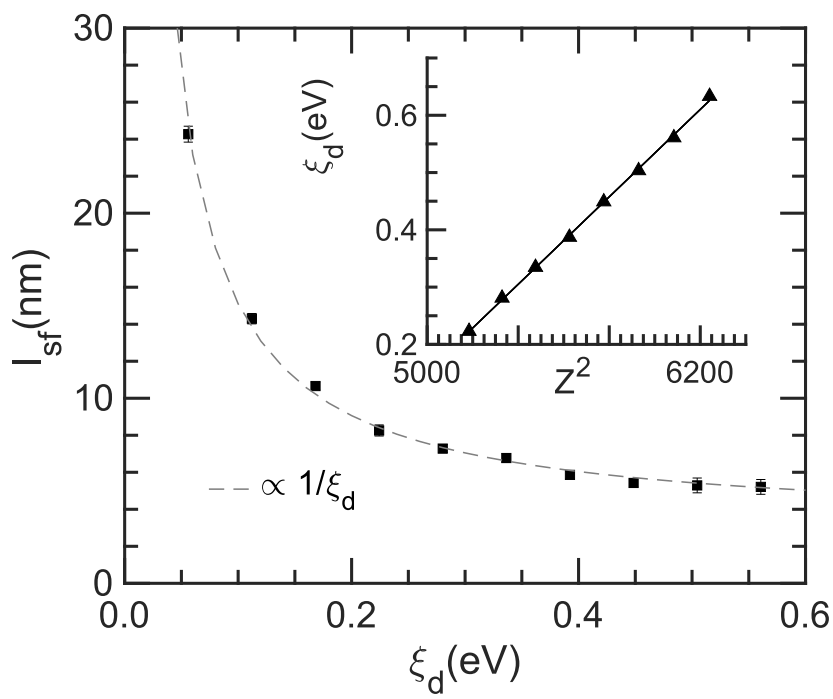

Figure 2.5: Spin flip diffusion length $l_{\mathrm{sf}}$ calculated as a function of the spin-orbit coupling constant $\xi_{d}$ for fcc Pt. Dotted grey lines indicate the $1 / \xi_{d}$ fit of $l_{\mathrm{sf}}$. Inset: $\xi_{d}$ as a function of the square of the atomic number $Z$ for the $5 d$ electrons.

\section{Spin-orbit coupling}

For the transition metals the spin-orbit splitting of the conduction $d$ bands that lie near the Fermi level plays an important role in spin transport. We plot the calculated $\xi_{d}$ for $5 d$ elements in the inset of Fig. 2.5 as a function of $Z^{2}$. Because the $5 d$ bands of Au are fully filled we choose Pt, the element with the next highest $\xi_{d}$ in the series, to study the scaling of $l_{\mathrm{sf}}$ vs. $\xi_{d}$. The SOC parameter $\xi$ is scaled by varying $\lambda_{s c}$ in (2.2) from $0-1$ in the selfconsistent calculation of the input potentials. Keeping the $\Delta$ corresponding to the room temperature resistivity of Pt constant, we perform calculations to extract $l_{\mathrm{sf}}$ and plot the results in Fig. 2.5 as a function of $\xi_{d}$. $l_{\mathrm{sf}}$ is found to be inversely proportional to $\xi_{d}$. For metals with intrinsic spin-orbit coupling, spin relaxation is achieved by mixing pairs of the Kramers degenerate spin states often quantified in terms of a spin-mixing parameter ' $b$ ' [23]. Fabian and Das Sarma [98] found that the spin relaxation rate $1 / \tau_{\text {sf }}$ scaled linearly with $b^{2}$ for a metal with free-electron like bands. Since the spin relaxation time $\tau_{\mathrm{sf}} \propto l_{\mathrm{sf}}^{2}$, the calculated trend plotted in the figure is consistent with their findings but now for a metal with a complex Fermi surface. While changes in lattice and band structure can make a straightforward application of Fig. 2.5 to predict the trend in $l_{\mathrm{sf}}$ across the $5 d$ series non-trivial, one would anticipate the trend to hold for elements with similar lattice that are neighbours. 


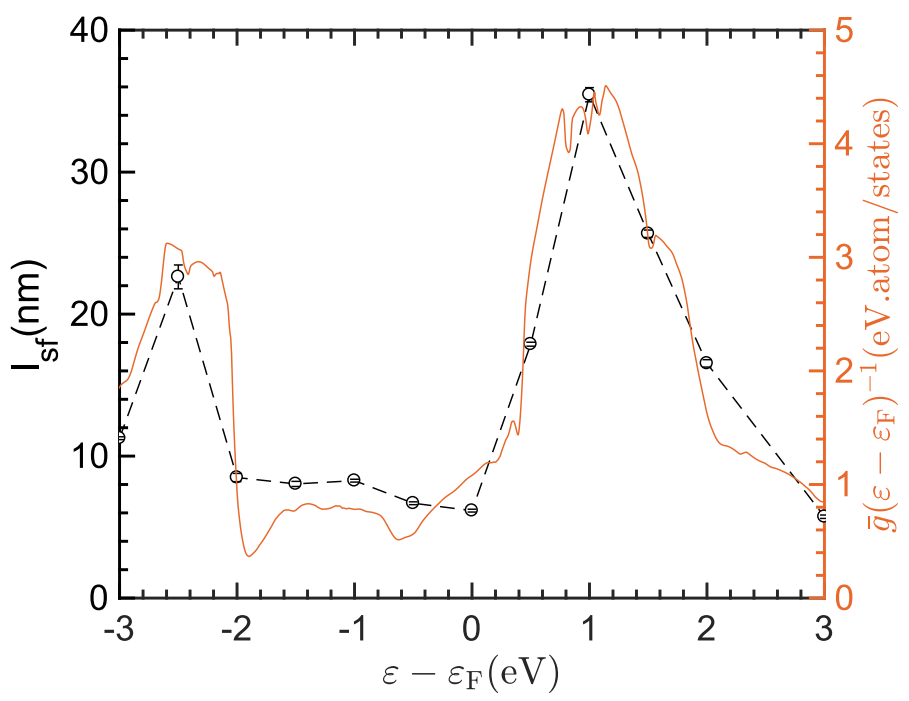

Figure 2.6: Spin flip diffusion length(black) and the inverse of density of states (orange) of Ta as a function of band filling

\section{Band-filling}

We first study the effect of filling of the $d$-bands taking a random example of bcc Ta. We keep the lattice disorder $\Delta$ constant corresponding to the room temperature resistivity of Ta. The Fermi energy is artificially moved through the bands from - 3 to $+3 \mathrm{eV}$ in steps of $0.5 \mathrm{eV}$ with respect to the self consistently calculated groundstate Fermi energy $\varepsilon_{\mathrm{F}}$ of Ta. Transport calculations are performed for each case. In Fig. 2.6 we plot $l_{\mathrm{sf}}$ vs. energy shift relative to the ground-state Fermi level. $l_{\mathrm{sf}}$ closely follows the inverse of the density of states (DoS), $\bar{g}\left(\varepsilon-\varepsilon_{F}\right)$ for each energy. This indicates that for materials displaying a sharp peek in DoS near the Fermi level, a significant change in spin quenching could be achieved by even small shifts in Fermi level through extrinsic factors like impurities, strain etc as is often the case with experimentally prepared samples.

From the forgoing discussion on SOC and band-filling, one might expect that the interplay between $\xi_{d}$ and DoS should determine the trend in $l_{\text {sf }}$ across the $5 d$ series. However, the RT values calculated for $l_{\mathrm{sf}}$ that are shown in Fig. 2.7 reveal that the dominant trend is determined by the inverse of the Fermi level density of states (DoS), $g\left(\varepsilon_{F}\right)$ even though the strength of the SOC triples across the series. Also shown in the figure in orange is $g\left(\varepsilon_{F}\right)$ averaged over an energy window of $\pm k_{B} T$ about the Fermi energy $\bar{g}\left(\varepsilon_{F}\right)$. For example, Ta and $\mathrm{W}$ are both bcc and as neighbours in the periodic table have very similar values of $\xi_{d}$ yet $l_{\mathrm{sf}}^{\mathrm{W}}$ is some five times larger than $l_{\mathrm{sf}}^{\mathrm{Ta}}$. A low DoS means fewer possibilities to scatter with a spin 


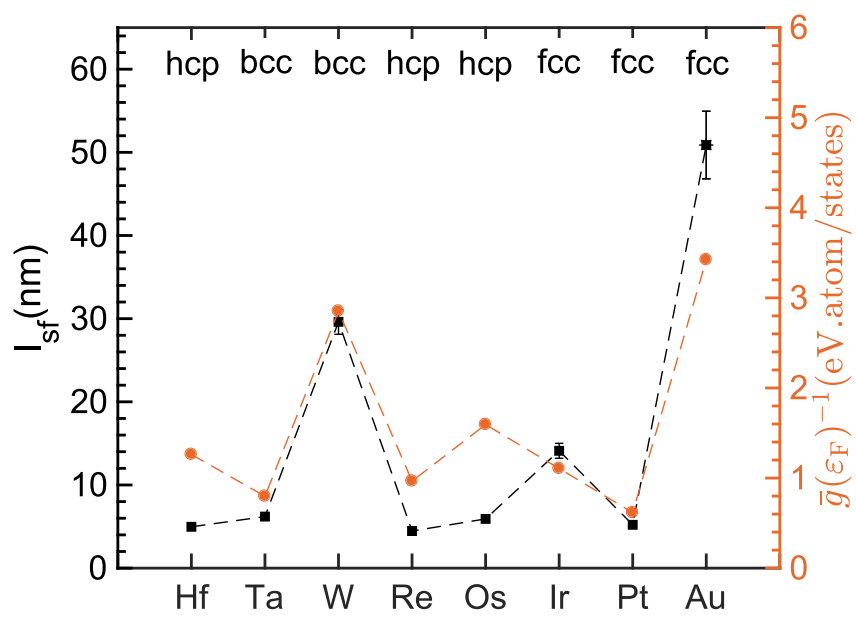

Figure 2.7: Black (left): spin-flip diffusion length $l_{\text {sf }}$ for $5 d$ transition metals calculated at room temperature $(300 \mathrm{~K})$, the error bars correspond to the spread of values for ten different configurations of thermal disorder. For hcp metals, $c$-axis values are shown. Orange (right): inverse of $\bar{g}\left(\varepsilon_{F}\right)$, the density of states averaged over an energy window of $\pm k_{B} T$ about the Fermi energy $\varepsilon_{F}$.

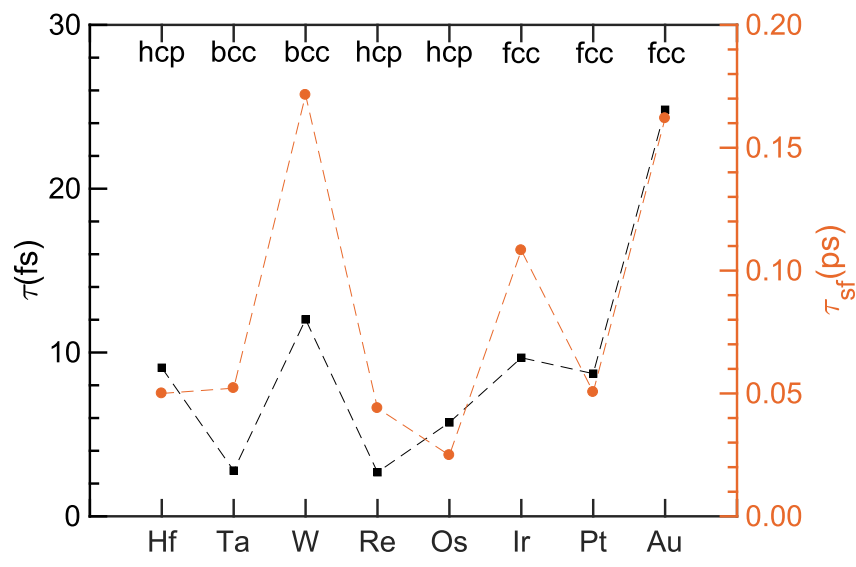

Figure 2.8: Room temperature values for relaxation times for momentum and spin flip scattering. For hcp metals, the value for transport along $c$ - axis is shown.

flip. Experimental values of $l_{\mathrm{sf}}$ reported at RT are 1.8 and $1.9 \mathrm{~nm}$ for bcc Ta, 2.1 $\mathrm{nm}$ for bcc W, between 1 and $11 \mathrm{~nm}$ for Pt and from 27 to $86 \mathrm{~nm}$ for Au [77]. The lack of any correlation with the "intrinsic" values we calculate suggests that the measurements are dominated by other factors. In spite of the apparent complexity of the relationship given in (2.18), Fig. 2.8 shows that the factor dominating the 
behaviour of both relaxation times is the inverse Fermi level density of states. We note that the electron-phonon coupling and phonon-modulated SOC effects enter our calculation implicitly via the resistivity $\rho$ while the density of states $\bar{g}\left(\varepsilon_{F}\right)$ is calculated with SOC explicitly included in the Hamiltonian.

\section{Temperature dependence}

Using $\Delta$ to reproduce experimental resistivities of the $5 d$ series metals available in literature $[62,91,92]$, we can study the temperature dependence of $l_{\mathrm{sf}}$ arising from electron-phonon coupling. In the equipartition regime, the resistivity is linear with temperature and our method of simulating lattice disorder using an uncorrelated Gaussian distribution is found to be suitable.

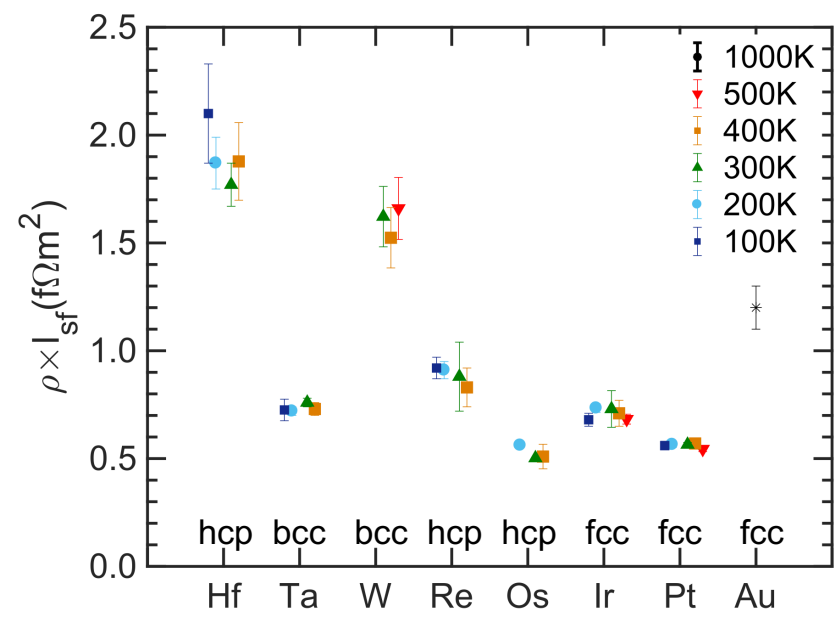

Figure 2.9: The product of spin flip diffusion length and resistivity for $5 d$ metals calculated at different temperatures, the error bars are estimated from the average spreads over 10 configurations of disorder for the calculations of $l_{\mathrm{sf}}$ and $\rho$.

For temperatures in the range 100-500 K, the product $\rho(T) l_{\mathrm{sf}}(T)$ is plotted for all $5 d$ elements in Fig. 2.9 where it is seen to be independent of temperature within the error bars of the calculations. (The large value of $l_{\mathrm{sf}}^{\mathrm{W}}$ at $300 \mathrm{~K}$ requires calculations with an excessively long geometry and values are only reported for the temperature range 300-500K.) This is in agreement with predictions made by Elliott and Yafet for doped semiconductors and alkali elements [23, 24] but now for Fermi surfaces that are far more complex than those they considered, for which their approximations are not applicable. As such, this result is non-trivial. For different elements, there is no obvious systematic variation in $\rho l_{\mathrm{sf}}$ as a function of $Z$. To determine $l_{\mathrm{sf}}^{\mathrm{Au}}$ at room temperature, we considered an elevated temperature 
of $T=1000 \mathrm{~K}$ and then assumed that $\rho(T) l_{\mathrm{sf}}(T)$ was a constant in order to estimate the RT value of $l_{\mathrm{sf}} \sim 50 \mathrm{~nm}$ shown in Fig. 2.7 (as a black asterisk)

\subsubsection{Spin Hall effect}

To examine the correlation seen in experiment between the $\Theta_{\mathrm{sH}}$ and $l_{\mathrm{sf}}$ [19], it is desirable to determine $\Theta_{\mathrm{sH}}$ using the same approximations as we used to calculate $l_{\mathrm{sf}}$. Most quantitative theoretical studies of the SHE [35, 99-101] are based upon the Kubo formalism and have focused on the so-called "intrinsic" contribution that does not consider the role of the electron-phonon scattering mechanism that dominates the resistivity of elemental metals at room temperature where the vast majority of determinations of $\Theta_{\mathrm{sH}}$ have been made [77]. In the linear response regime, the scattering theory we use is equivalent to the Kubo formalism [102] and therefore includes the intrinsic contribution. The advantage of the scattering formalism is that intrinsic and extrinsic mechanisms are included on equal footings. By calculating the transverse spin current resulting from a longitudinal

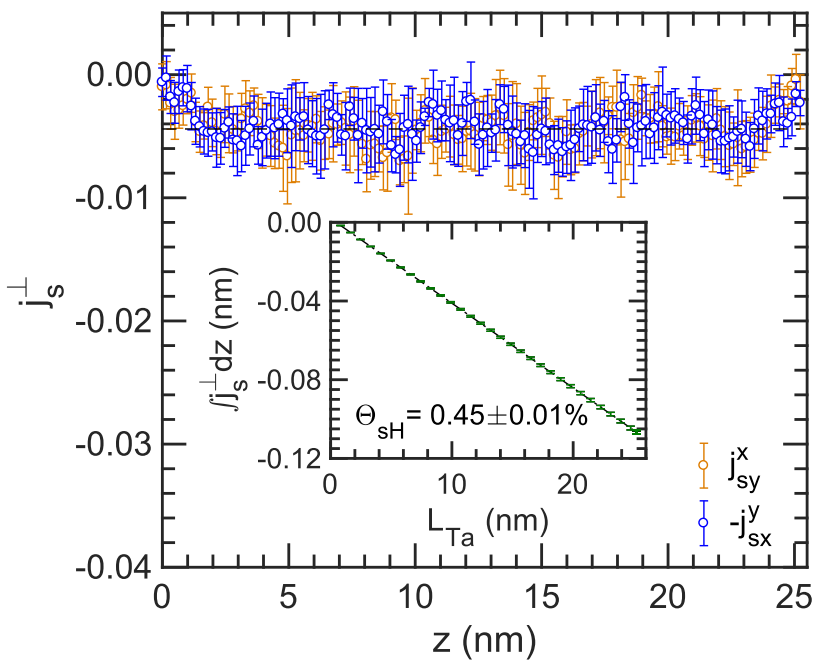

Figure 2.10: Planar averaged transverse spin currents plotted as a function of the $z$ coordinate calculated using an $s p d$ basis for $7 \times 7$ supercell with a BZ sampling of $23 \times 23 k$ points. Inset: integrated transverse spin current as a function of the length $\mathrm{L}_{\mathrm{Ta}}$ of the Ta slab. Error bars are estimated from the average deviation over 10 configurations of random disorder.

charge current using the local current method [20] introduced previously to study $\Theta_{\mathrm{sH}}^{\mathrm{Pt}}$ [34], we can determine $\Theta_{\mathrm{sH}}$ as a function of temperature for all $5 d$ metals. An example of $\Theta_{\mathrm{sH}}$ calculation at RT is shown in Fig. 2.10 for the case of Ta where we inject charge current from ballistic Ta leads and calculate the average of the trans- 
verse spin currents as a function of the $z$ coordinate. The transverse spin currents can be integrated along the transport direction to separate out bulk (slope) and lead (intercept) contributions to $\Theta_{\mathrm{sH}}$ using a linear fit[34] as shown in the inset to Fig. 2.10.

\section{Temperature dependence}

$\Theta_{\mathrm{sH}}$ scales linearly with the resistivity $\rho(T)$ as shown in Fig. 2.11 to a reasonable approximation. For most metals, deviations from linearity are visible for the lowest resistivity values shown at $T \sim 100 \mathrm{~K}$. The biggest discrepancy is for Re. Linearity of $\Theta_{\text {SH }}$ vs. $\rho$ implies a weak temperature dependence of the spin Hall conductivity $\sigma_{\mathrm{sH}} \equiv \Theta_{\mathrm{sH}} / \rho$ as shown in Fig. 2.12. Our finding of a weak temperature dependence
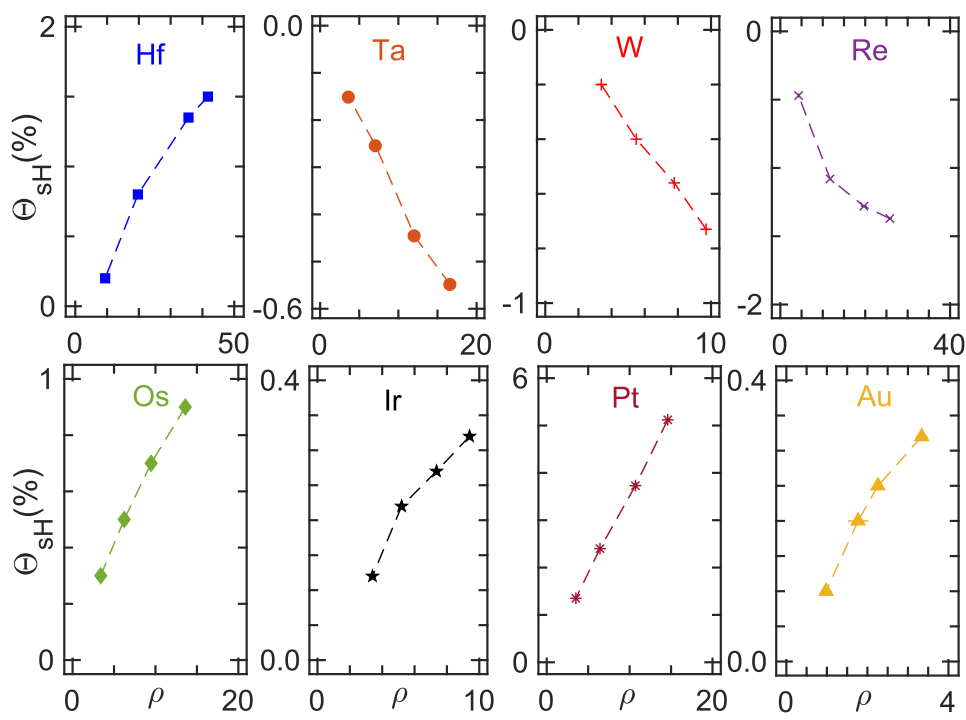

Figure 2.11: Spin Hall angle $\Theta_{\mathrm{sH}}$ as a function of resistivity $\rho$ for all $5 \mathrm{~d}$ metals. Dotted lines are guide for the eye. Error bars in the estimation of $\Theta_{\mathrm{sH}}$ and $\rho$ included as vertical and horizontal bars respectively are smaller than the symbols in most cases.

of $\sigma_{\mathrm{sH}}$ is in qualitative agreement with the temperature independence found for $\mathrm{Pt}$ by Isasa et al. [103, 104] who, however, reported a substantially lower value of $\sigma_{\mathrm{sH}} \sim 1200(\Omega \mathrm{cm})^{-1}$ (note that we use the $\hbar / 2 e$ convention [105]). A subsequent measurement by the same group [88] yielded a room temperature spin Hall conductivity of $\sim 3200(\Omega \mathrm{cm})^{-1}$ in excellent agreement with our value. At low temperatures in clean samples, they observed an enhancement of $\sigma_{\mathrm{sH}}$ with decreasing temperature. Such an enhancement was recently found [106] in calculations in which phonon modes of Pt were explicitly populated as a function of temperature 
[61]. It was pointed out that the temperature independence of $\sigma_{\mathrm{sH}}$ is characteristic of the classical equipartition regime [106]. At temperatures below the Debye temperature our description of thermal disorder in terms of a Gaussian distribution of uncorrelated atomic displacements is not suitable for studying the weak scattering limit.

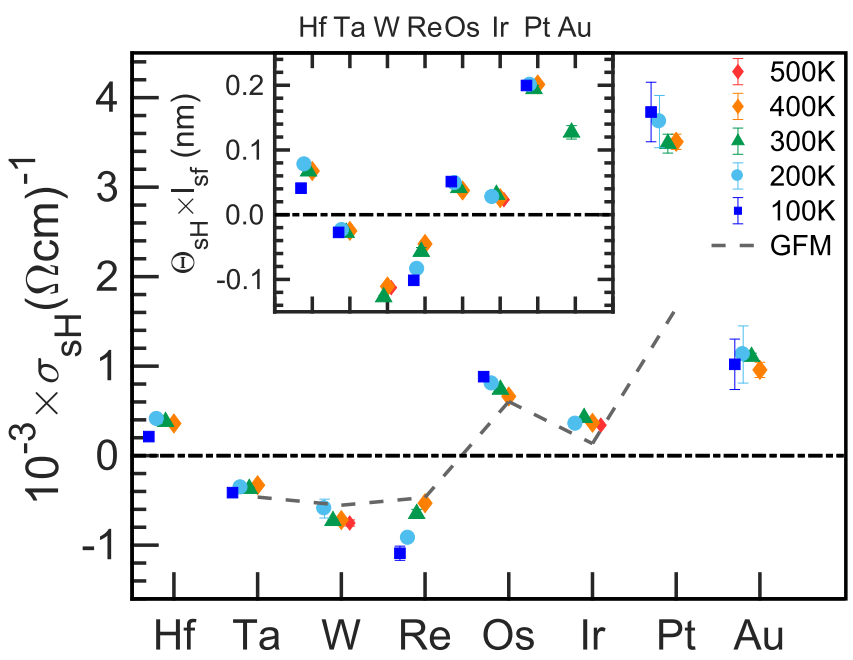

Figure 2.12: The spin Hall conductivity $\sigma_{\mathrm{sH}}\left(=\Theta_{\mathrm{sH}} \times \sigma\right)$ calculated at different temperatures. The room temperature spin Hall conductivity for all $5 d$ elements (except $\mathrm{Hf}$ and $\mathrm{Au}$ ) reported by Tanaka et al. [35] using a Green function method (GFM) and a phenomenological "quasiparticle damping rate" to account for disorder is included for comparison. The inset shows the product $\Theta_{\mathrm{sH}} \times l_{\mathrm{sf}}$ as a function of temperature. Only one value is plotted for Au based on an extrapolation of $l_{\mathrm{sf}}$ to RT.

The element $(Z)$ dependence is in qualitative agreement with the linear response calculations by Tanaka et al. who used an empirical "quasiparticle damping parameter" to represent disorder [35]. In these latter calculations, the correct bcc, fcc and hcp structures were used for each element but $\mathrm{Hf}$ and Au were not considered. For hcp Hf we find a small, positive value of $\sigma_{\mathrm{sH}}$ in agreement with recent experiments [107] but at variance with explicit calculations for hcp Hf [107, 108]. At this point, we note that for materials where the Fermi level lies close to degeneracies or peaks in the DoS, estimated values of $\sigma_{\mathrm{sH}}$ can be sensitive to fine details of the band structure and accurate determination of the Fermi level. This will be carefully elucidated in a following section using a rigid band analysis.

In Ref. [34], $\Theta_{\mathrm{sH}}^{\mathrm{Pt}}$ was found to be proportional to the resistivity $\rho$ whereas $l_{\mathrm{sf}}^{\mathrm{Pt}}$ was proportional to the conductivity $[61,86]$. The product $\Theta_{\mathrm{sH}} \times l_{\mathrm{sf}}$ is shown in 
the inset to Fig. 2.12 for all $5 d$ elements for a number of temperatures between 100 and $500 \mathrm{~K}$. As a function of $Z$, it is seen to follow the trend of $\sigma_{\mathrm{sH}}$ and as a function of temperature it is approximately constant for all metals. What remains to be studied is whether this relationship holds for different scattering mechanisms and whether a Matthiessen-like composition rule holds for the spin Hall effect and spin-flip scattering.

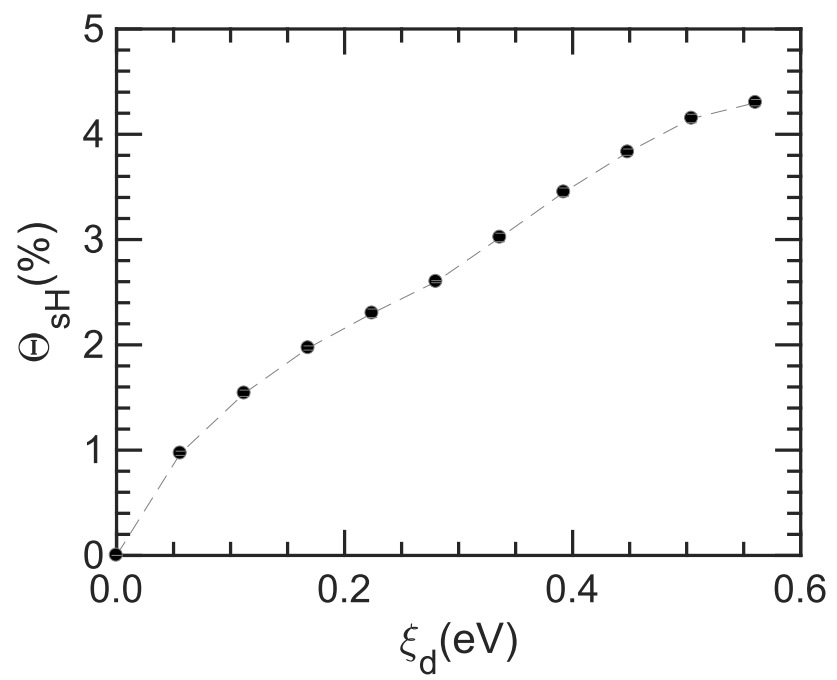

Figure 2.13: Spin Hall angle $\Theta_{\mathrm{sH}}$ for fcc Pt vs. spin-orbit coupling parameter $\xi_{d}$. Dotted line is a guide for the eye. Error bars are smaller than the symbols.

\section{Spin-orbit coupling}

The largest value of $\Theta_{\mathrm{sH}}$ in the $5 d$ series found for Pt can be attributed to its strong spin-orbit coupling. Here we examine how $\Theta_{\mathrm{sH}}^{\mathrm{Pt}}$ scales with the strength of the spinorbit coupling. The spin Hall currents are calculated for Pt and the SOC coupling constant $\xi_{d}$ is varied like we did for $l_{\text {sf }}$ in Sec. 2.3.1 but now with unpolarized leads. We plot $\Theta_{\mathrm{sH}}$ as a function $\xi_{d}$ in Fig. 2.13. As expected, $\Theta_{\mathrm{sH}}$ increases with the strength of $\xi_{d}$. The non-linear variation is in qualitative agreement with the findings of Tanaka et al. [35].

\section{Band-filling}

Based upon a rigid band study for the fcc structure, Guo identified a switch from positive values of $\sigma_{\mathrm{sH}}$ for a band filling corresponding to Pt to negative values for band fillings corresponding to Ta and $\mathrm{W}$ [99] and this has been interpreted in terms of Hund's third rule [109]. Here we calculate $\Theta_{\mathrm{sH}}$ for all three structures and examine the results shown in Fig. 2.14 for conclusive evidence for a correspondence 


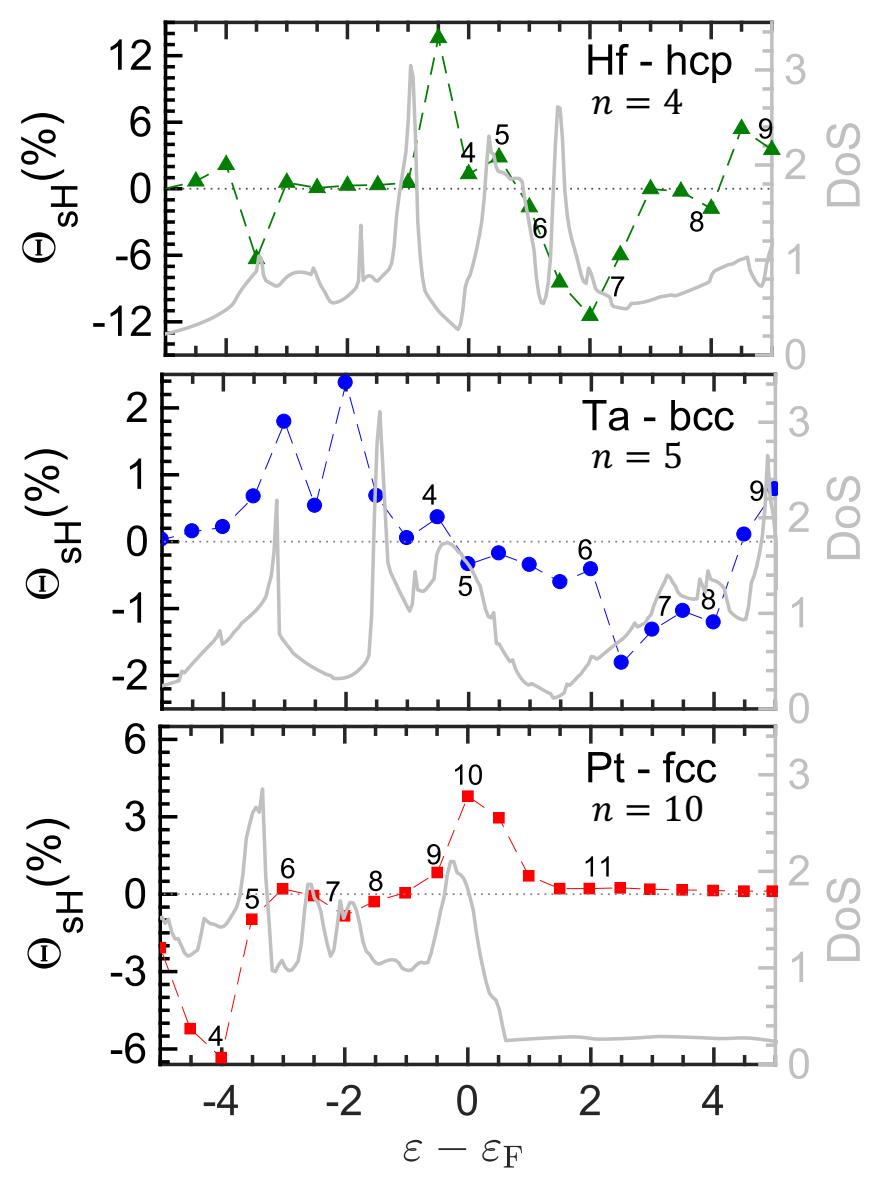

Figure 2.14: Rigid band analysis of $\Theta_{\mathrm{sH}}$ for Hf (green), Ta (blue) and Pt (red) for $\Theta_{\mathrm{sH}}$. Spin Hall angle $\Theta_{\mathrm{sH}}$ calculated as a function of the energy $\varepsilon$. Assuming a single $s$ electron across the whole series, the equivalent band filling $n$ corresponding to an outer $d^{n-1} s^{1}$ configuration at different energies calculated using the number of states (NoS) function is indicated on the plot. The density of states (DoS) per atom for each structure is plotted on the right. $\Theta_{\mathrm{sH}}$ values for Pt are reproduced from Ref [20].

between $\sigma_{\mathrm{sH}}$ and Hund's rule. For different band fillings $\Theta_{\mathrm{sH}}$ was calculated for each structure hcp Hf, bcc Ta and fcc Pt by artificially varying the Fermi level in steps of $0.5 \mathrm{eV}$ in a window of $-5 \mathrm{eV}$ to $+5 \mathrm{eV}$ around the Fermi energy $\varepsilon_{\mathrm{F}}$. The lattice disorder was kept constant corresponding to the appropriate room temperature resistivity. The filling of the conduction band at different energies is calculated from the number of states (NoS) given by $N(\varepsilon)=\int_{-\infty}^{\varepsilon} g\left(\varepsilon^{\prime}\right) \mathrm{d} \varepsilon^{\prime}$. Our calculations reproduced the peaks observed by Guo et al. as a function of band filling for the 

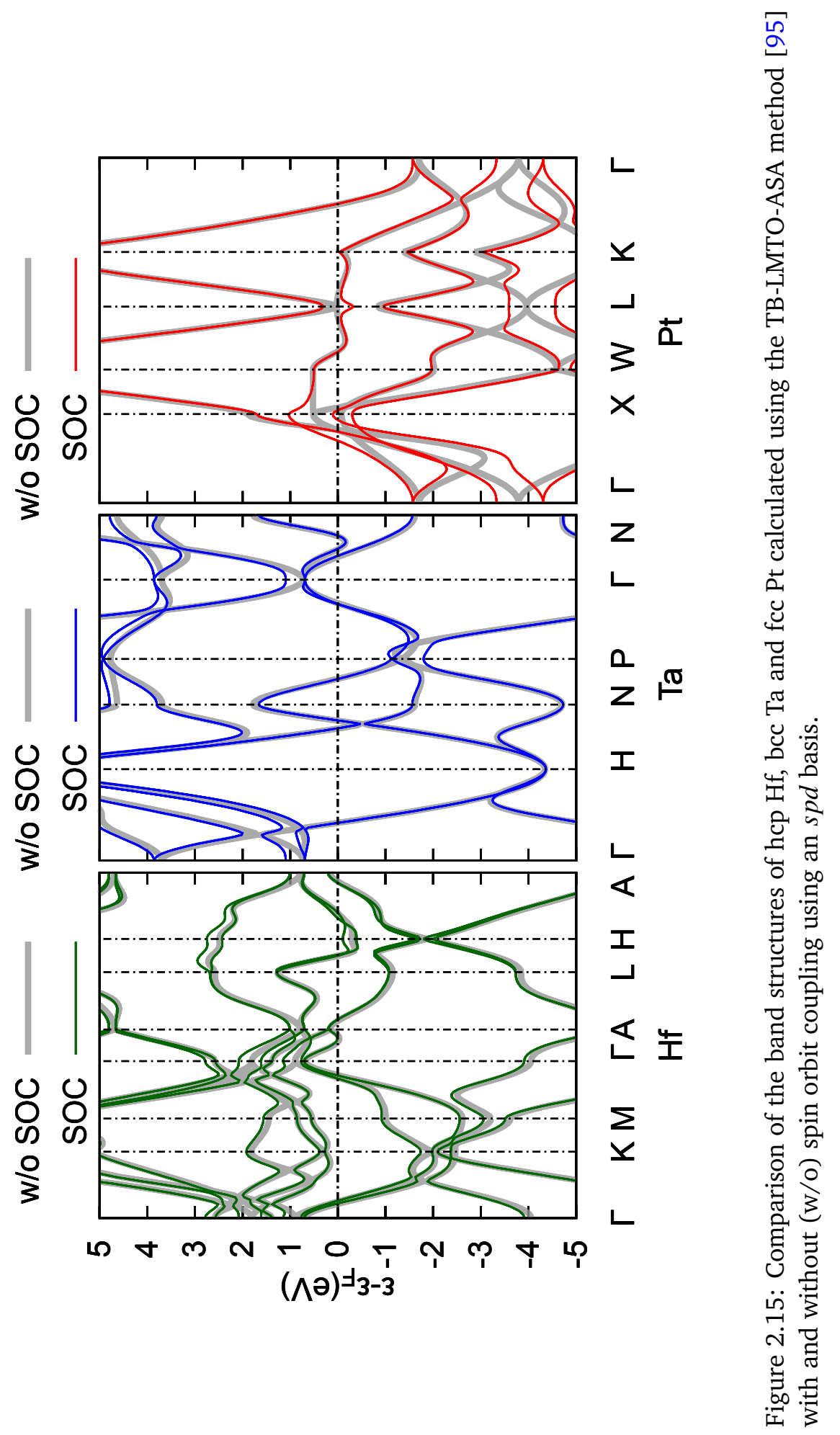
fcc Pt structure. We see that the trends predicted for different crystal structures are only roughly in qualitative agreement. In spite of the lack of exact coincidence, the peaks and general variation in $\Theta_{\mathrm{sH}}(N(\varepsilon))$ seems to be driven by DoS $(\varepsilon)$ for all three band structures. However, there are clear discrepancies in the sign and magnitude of $\Theta_{\mathrm{sH}}$ predicted by each structure for different fillings. The biggest of them being a significant negative value predicted for hcp Hf using an fcc Pt band structure and a giant negative value predicted for hcp Re using an hcp Hf structure, the latter being surprising on account of the similarity of the lattices. Furthermore, the sign of hcp Os is predicted incorrectly using all three structures. It is worthwhile noting that we found a switch in the sign of $\Theta_{\mathrm{sH}}$ for transport perpendicular to the hexagonal axis in Os which will be presented in Sec. 2.3.3.

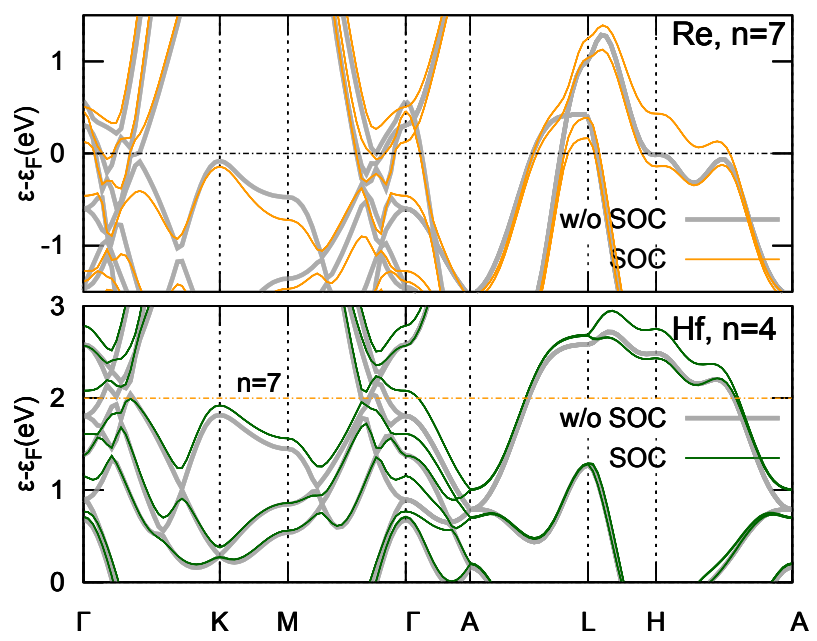

Figure 2.16: Band structures with and without spin-orbit coupling (SOC) for hcp (top) Re and (bottom) Hf. The dotted horizontal orange line at $2 \mathrm{eV}$ in dispersion of $\mathrm{Hf}$ corresponds to the 6 conduction electrons of for $\operatorname{Re}\left(d^{6} s^{1}\right)$.

The peaks observed as a function of band filling for fcc Pt originate in the high symmetry $\mathrm{P}$ and $\mathrm{X}$ points of the Brillouin zone with degeneracies that are strongly affected by SOC. The position of these degeneracies depends on the lattice structure as evident from the comparison in Fig. 2.15. We note that the two giant peaks in $\Theta_{\mathrm{sH}}^{\mathrm{Hf}}$ coincide with the double degenerate states between $\mathrm{L}-\mathrm{H}$ at $\varepsilon-\varepsilon_{\mathrm{F}}=-0.5$ $\mathrm{eV}$ and $2.5 \mathrm{eV}$ and the complex of five quasi degenerate states at $\Gamma-\mathrm{K}$ points lifted by SOC. In Ta, the negative peak in $\Theta_{\mathrm{sH}}^{\mathrm{Ta}}$ occur at the three-fold degeneracy on $\mathrm{P}$ around $-2 \mathrm{eV}$ while the positive peak does not coincide with any splitting. The rigid band analysis presented here can not capture the effect of a change in the 
band structure as it assumes only the Fermi level to simply move up as we move along the series from Hf to Au. However, a change in atomic volume could introduce significant distortions even for similar lattices as shown in Fig. 2.16 where we compare bands of two hcp metals Hf and Re. The Fermi level in Re lies slightly above the degeneracies lifted by SOC near $\Gamma-\mathrm{K}$ and $\mathrm{M}-\Gamma-\mathrm{A}$ in comparison with energy value of $2 \mathrm{eV}$ in $\mathrm{Hf}$ bands that corresponds to a nominal Re filling which explains a significantly low value of $\Theta_{\mathrm{sH}}^{\mathrm{Re}}$ than predicted from Hf-bands.

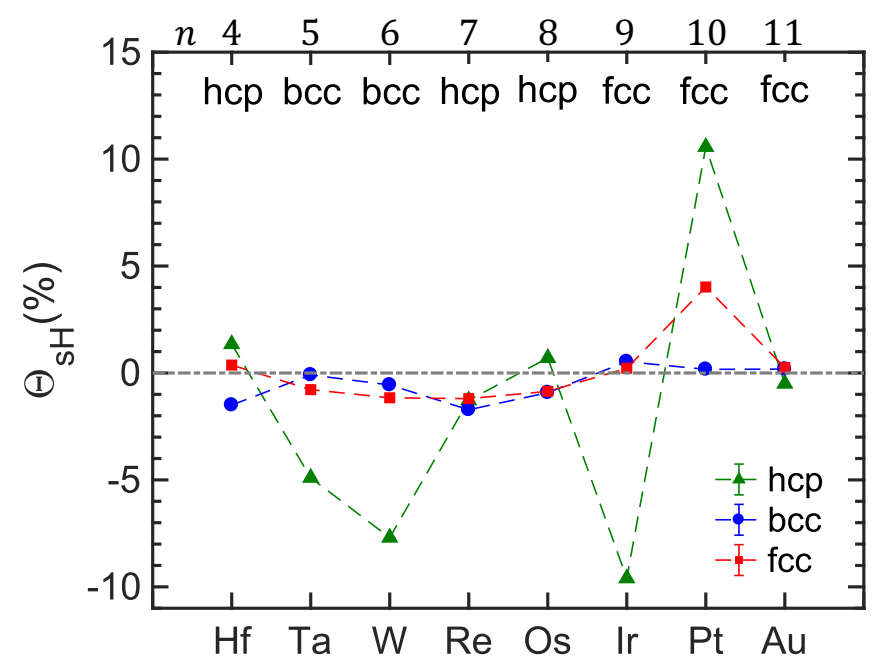

Figure 2.17: Comparison of $\Theta_{\mathrm{sH}}$ calculated for all elements in the hcp, bcc and fcc structures where for each element we use its observed atomic volume (Wigner-Seitz sphere radius). The lattice disorder $\Delta$ reproduces the room temperature resistivity of each metal in its observed structure given on the top axis. For the hcp structures, the charge current is along the $c$-axis. For the hcp structures of elements other than Hf, Re and Os, the ideal ratio $c / a=\sqrt{8 / 3}$ is used. The number of conduction electrons $n$ for each element is indicated on the top.

Finally, the role played by the crystal structure in combination with the effect of band filling and atomic volume across the series can be studied by explicitly calculating $\Theta_{\mathrm{sH}}$ using hcp, bcc and fcc structures for each of the $5 d$ metal elements. We calculated $\Theta_{\mathrm{SH}}$ keeping the atomic volume and thermal disorder $\Delta$ constant and equal to the values corresponding to the original crystal structure of each element. The results are plotted in Fig. 2.17. For the hcp structure $\Theta_{\mathrm{sH}}$ exhibits an increase in magnitude as we move from $\mathrm{Hf}(n=4)$ to $\mathrm{W}(n=6)$ but the small positive peak seen in Fig. 2.14 for Ta $(n=5)$ is not reproduced. Another important feature is that the signs of $\Theta_{\mathrm{sH}}$ for bcc Hf, hcp Ir, fcc and bcc Os and hcp Au is at variance with the predictions based on $d$ band filling [35] ruling out the general applicability of 
Hund's third rule for determining the sign of $\Theta_{\mathrm{sH}}$. Our results prove that the sign of $\Theta_{\mathrm{sH}}$ depends not only on the band filling but also on the structure.

\subsubsection{Anisotropy}

Hf, Re and Os have hexagonal close packed (hcp) crystal structures which leads to anisotropic scattering rates in the directions parallel $(\|)$ and perpendicular $(\perp)$ to the hexagonal crystal axis (0001). The anisotropy in the resistivity of single crystal samples of Re between 77 - $1600 \mathrm{~K}$ [91] and Os between 290 -1600 K [110] are reported by experiments while we are not aware of any experimental studies on the anisotropy in Hf. We calculate anisotropic spin transport properties for the hcp crystals at RT by setting the $z$ direction along $0001(\|)$ and $\overline{1} 2 \overline{1} 0(\perp$ ) directions. We reproduce the experimental anisotropy in the resistivity of Re, $\rho_{\perp} / \rho_{\|} \sim 1.3$ at RT while that in Os is $\rho_{\perp} / \rho_{\|} \sim 1.1$, underpredicted in comparison with the experimental measurements, $\rho_{\perp} / \rho_{\|} \sim 1.5[110,111]$. However, our values are in agreement with calculated anisotropy [112] using a Boltzmann approach [113] including electron-phonon coupling. Of the three hcp metals in the $5 \mathrm{~d}$ series, Hf exhibits the highest anisotropy $\rho_{\perp} / \rho_{\|} \sim 1.7$ at RT. RT values for all relevant transport properties discussed in this work given in Table 2.1 includes directional dependent values $(\|$ and $\perp$ ) for hcp metals in the series. Our calculated values of $\Theta_{\mathrm{sH}}$ are not in agreement with the anisotropy predicted in Ref. [108] where a FLAPW implementation of the Kubo-formalism was used. We find that $\Theta_{\mathrm{sH}}$ for the two directions are approximately equal in magnitude but have opposite signs.

\subsection{Other calculations}

Very little can be said about accuracy (i) in the absence of consensus about the values of $l_{\mathrm{sf}}$ and $\Theta_{\mathrm{sH}}$ reported in experiments and (ii) because other calculations that do not include realistic thermal disorder cannot be rigorously compared with our results. However, the robustness of the results presented in this work can be tested by studying the effect of computational parameters such as supercell size, BZ sampling, basis and number of terms in the SOC integral etc. on our calculations. An in-depth analysis of such an exercise for the case of fcc Pt that yielded best estimates of $l_{\mathrm{sf}}^{\mathrm{Pt}}=5.3 \pm 0.4 \mathrm{~nm}$ and $\Theta_{\mathrm{sH}}^{\mathrm{Pt}}=(4.5 \pm 1) \%$ were presented in Ref [20]. To reinforce the conclusions drawn there, we consider other examples here.

\subsubsection{Au}

$\mathrm{Au}$ has a long mean free path at room temperature as seen in Table 2.1 and consequently a longer spin flip diffusion length which which makes it necessary to use a very long scattering region to extract a reliable value of $l_{\mathrm{sf}}$ following (2.19). Hence, we perform the $l_{\mathrm{sf}}$ calculation only at a higher temperature $(1000 \mathrm{~K})$ where 


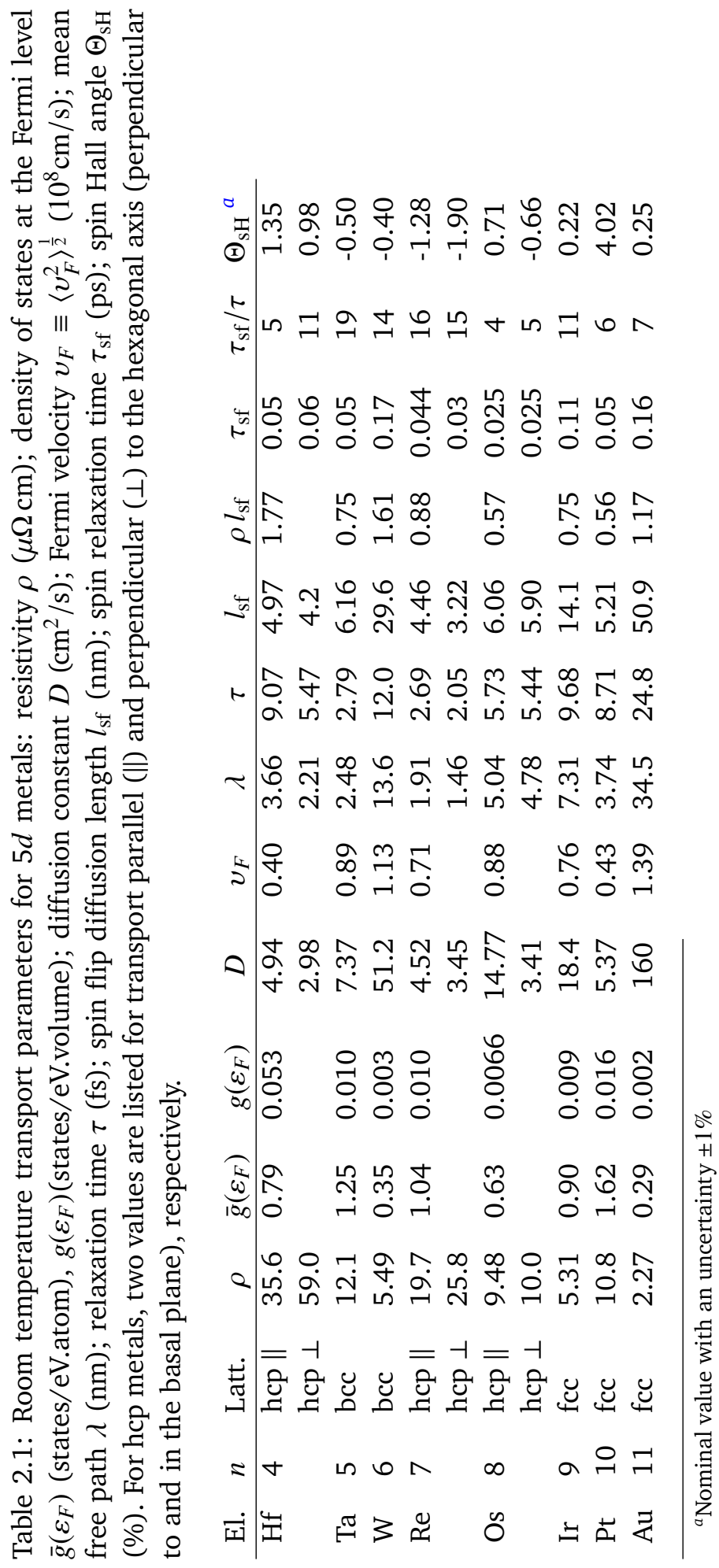




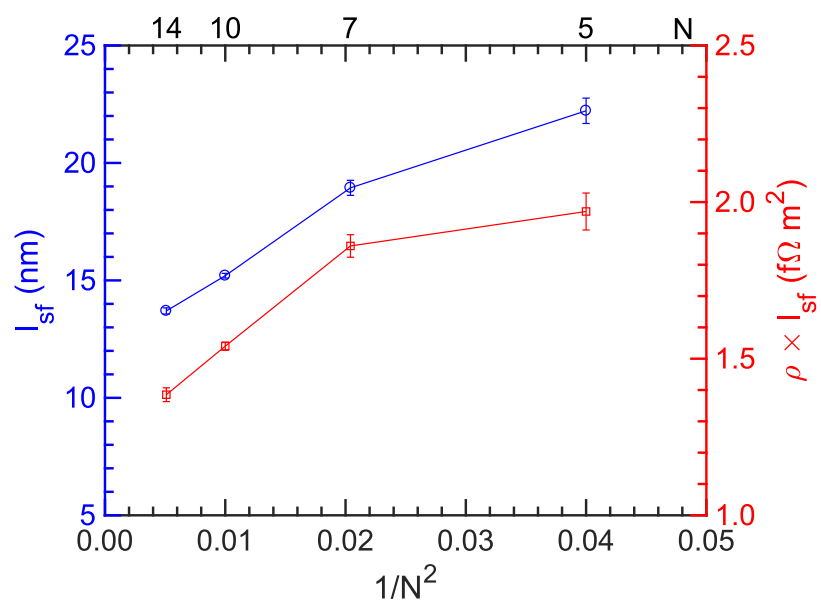

Figure 2.18: Convergence of the spin flip diffusion length $l_{\mathrm{sf}}$ (lhs) and $\rho \times 1_{\mathrm{sf}}$ (rhs) of Au with respect to the inverse of supercell size, $N \times N$.

the length scale is tractable using our computational scheme and use the product $\rho \times l_{\text {sf }}$ estimated at this temperature to predict the $l_{\text {sf }}$ at room temperature. We plot $l_{\mathrm{sf}}^{\mathrm{Au}}$ as a function of the inverse of the size $\left(N^{2}\right)$ of the later supercell calculated at $1000 \mathrm{~K}$ in Fig. 2.18 in blue.

Only few electrons scatter at large angles $\sim 90^{\circ}$ with respect to the $z$ direction. Therefore it is not critical to have a lateral supercell larger than the the mean free path to reproduce 'bulk' behaviour. For e.g. it has been shown for Pt that it is sufficient to use a supercell of size $7 \times 7$ that is $\sim 2 \mathrm{~nm}$ thick. Because of the longer mean free path, the value of $l_{\mathrm{sf}}^{\mathrm{Au}}$ is found to depend significantly on the size of the lateral supercell unlike in other $5 d$ element as shown in Fig. 2.18. We found the the resistivity $\rho^{\mathrm{Au}}$ to be less sensitive to the SC size compared to the $l_{\mathrm{sf}}^{\mathrm{Au}}$. Therefore, $\rho \times l_{\text {sf }}$ follows the same behaviour as $l_{\text {sf }}$ as seen in Fig. 2.18 (red). Using Fig. 2.18, we extrapolate $\rho \times l_{\text {sf }}$ for an infinitely large supercell that would ideally represent a bulk system. The reported value of $\rho \times l_{\mathrm{sf}}$ for Au is the mean of the best calculated value and the value extrapolated to $N=\infty$ i.e. $1 / N=0$ with an uncertainty interval that spans between these two values.

\subsubsection{Ta}

There are two ways of generating AS potentials self-consistently (i) using the Stuttgart LMTO-ASA code (without SOC) $[64,96]$ and (ii) Questaal suite (with SOC)*. The generated potentials and the self consistently determined Fermi energies are then

\footnotetext{
" "Im" extension of the Stuttgart LMTO code that treats non-collinear magnetization and spin-orbit coupling is maintained in the Questaal suite at https://www.questaal.org.
} 
used in the transport code where SOC is added to the Hamiltonian as a perturbation. The values of $\Theta_{\mathrm{sH}}$ and $l_{\mathrm{sf}}$ presented in this work so far were calculated using the potentials generated by the Stuttgart code. $\Theta_{\mathrm{sH}}$ is sensitive to the Fermi energy in a rigid band structure as different bands contribute differently, see Fig. 2.14. The Fermi energies determined self-consistently with and without SOC are slightly different. Calculations for a fixed Fermi energy are valid for the products $\rho \times l_{\text {sf }}$, $\Theta_{\mathrm{sH}} \times l_{\mathrm{sf}}$ in the sense that they are a constant.

Table 2.2: Dependence of $l_{\mathrm{sf}}$ and $\Theta_{\mathrm{sH}}$ of Ta on basis set and three centre terms in the SOC Hamiltonian in the transport code for the cases where ground-state is calculated self-consistently with and without (w/o) spin-orbit coupling.

\begin{tabular}{lcccc}
\hline \hline & & \multicolumn{2}{c}{$s p d$} & $s p d f$ \\
\cline { 3 - 5 } & & 2 centre & 3centre & 2 centre \\
\hline \multirow{2}{*}{$l_{\text {sf }}(\mathrm{nm})$} & SOC & $5.96 \pm 0.39$ & $6.73 \pm 0.14$ & $6.01 \pm 0.30$ \\
& w/o SOC & $6.58 \pm 0.13$ & $6.78 \pm 0.12$ & $6.16 \pm 0.13$ \\
\hline \multirow{2}{*}{$\Theta_{\mathrm{sH}}(\%)$} & SOC & $-0.22 \pm 0.02$ & $-0.34 \pm 0.01$ & $0.077 \pm 0.01$ \\
& w/o SOC & $-0.45 \pm 0.01$ & $-0.53 \pm 0.01$ & $-0.074 \pm 0.01$ \\
\hline
\end{tabular}

In Table 2.2 we compare calculated values of $l_{\mathrm{sf}}$ and $\Theta_{\mathrm{sH}}$ for Ta with and without SOC included self-consistently for determining the ground state Fermi level. We find that the change of the basis from $s p d$ to $s p d f$ bring about negligible change in $l_{\text {sf }}$ when SOC is included self-consistently whereas including three centre terms in the SOC Hamiltonian in the transport code causes the largest variation in $l_{\mathrm{sf}}$. As a rule of thumb, computations involving matrix multiplications scales as the cube of the size of the matrix, a change in basis from $s p d$ (9 orbitals) to $s p d f$ (16 orbitals) thus more than quadruples the computational cost. It is thus preferred to use a minimal basis set wherever possible to reduce computational effort in transport calculations. Additionally, the use of a three centre term in the SOC integral increases computational cost by 70\% [57]. Given the overall variation in the predicted $l_{\mathrm{sf}}$ is only $\pm 0.66 \mathrm{~nm}$ around a mean value of $6.4 \mathrm{~nm}$, using an $s p d$ basis without including SOC is found to be a reasonable choice even for a less close-packed structure like bcc using our tight binding LMTO scheme.

Calculated value of $\Theta_{\mathrm{sH}}^{\mathrm{Ta}}$ presented in Table 2.2 vary between 0.08 and $-0.5 \%$. On account of the very small value of $\Theta_{\mathrm{sH}}$, it is found to be very sensitive to computational parameters within the numerical errors of our scheme. A best value of $(-0.16 \pm 0.21) \%$ for the $\Theta_{\mathrm{sH}}$ of Ta is obtained from the calculations with SOC terms. Based on the sensitivity analysis of $\Theta_{\mathrm{sH}}^{\mathrm{Ta}}$ and $\Theta_{\mathrm{sH}}^{\mathrm{Pt}}$ presented here and in Ref [20] respectively, we attach an uncertainty of about one percent to the calculated values of $\Theta_{\mathrm{sH}}$ given in Table 2.1. 


\subsection{Summary and Conclusions}

An in-depth first principles study on spin-orbit coupling (SOC) related bulk transport properties is presented for the $5 d$ transition metals including realistic temperature disorder. We provided a systematic description of the effect of SOC, density of states, anisotropy and band structure on spin transport properties and quantified all the relevant bulk parameters of electronic transport in $5 d$ metals. We find that the density of states (DoS) at the Fermi level plays the most dominant role in determining the trend of spin relaxation in the series providing opportunities to tune $l_{\mathrm{sf}}$ by alloying or introducing defects. A band filling analysis revealed that the DoS drives the qualitative trend of $\Theta_{\mathrm{sH}}$ while the relative position of the Fermi level with respect to the SOC split band-degeneracies plays the most significant role in determining the quantitative values for $\Theta_{\mathrm{sH}}$. Contrary to common assumptions, the sign of $\Theta_{\mathrm{sH}}$ does not follow Hund's third rule but instead the crystal structure plays a crucial role. For example we find a positive value for $\Theta_{\mathrm{sH}}^{\mathrm{Hf}}$ and switch in sign for $\Theta_{\mathrm{sH}}^{\mathrm{Os}}$ between transport parallel and perpendicular to the c-axis. In general the spin Hall conductivity is found to be weakly dependent on temperature in the equipartition regime.

In addition to $l_{\mathrm{sf}}$ and $\Theta_{\mathrm{sH}}$ the benchmark values of the products $\rho \times l_{\mathrm{sf}}$ and $\Theta_{\mathrm{sH}} \times l_{\mathrm{sf}}$ provided in this chapter could guide in the experimental extraction of spin transport parameters of transition metals at relevant temperatures. Our findings should improve the current description of spin transport in materials and accelerate the search for candidate materials for spintronics applications. 
Spin transport in $5 d$ transition metals 


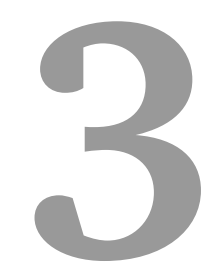

\section{Transverse spin currents in Fe-Ni systems ${ }^{* \dagger}$}

First-principles quantum transport calculations using scattering theory predict large transverse spin current generation in bulk $\mathrm{Ni}_{x} \mathrm{Fe}_{1-x} 3 d$ ferromagnetic (FM) alloys. The spin Hall angle $\Theta_{\mathrm{sH}}$ and spin-swapping angle $\Theta_{\mathrm{ss}}$ calculated as a function of the stoichiometry $x$ show only a weak temperature dependence in the strong alloy regime. We calculate a maximum value of $\Theta_{\mathrm{sH}} \sim 7.5 \%$ and $\Theta_{\mathrm{ss}}=-2.5 \%$ for the Ni end member of the series at room temperature. A qualitative model based on Mott scattering provides good agreement with the calculated results indicating that skew scattering may be the dominant mechanism leading to transverse spin currents in these systems. Based on the angular dependence of $\Theta_{\mathrm{sH}}, \Theta_{\mathrm{ss}}$ and $\Theta_{\mathrm{aH}}$, the anomalous Hall angle, we demonstrate how magnetization of the FMs offers a handle to manipulate bulk generated transverse spin and charge currents.

\subsection{Introduction}

When a charge current $\mathbf{j}_{c}$ flows in a material, then spin-orbit coupling (SOC) gives rise to a spin current $\mathbf{j}_{s}^{\hat{\mathbf{n}}}$ in the transverse direction $\hat{\mathbf{n}}$ such that spin-up and spindown electrons polarized in the $\mathbf{j}_{s} / j_{s}$ direction perpendicular to both $\mathbf{j}_{c}$ and $\hat{\mathbf{n}}$ accumulate at opposite edges of a sample, Fig. 3.1(a). This is the spin Hall effect (SHE)

\footnotetext{
"To be submitted as: R. S. Nair, K. Gupta, Z.Yuan, P. J. Kelly, "Transverse spin currents in bulk Fe-Ni systems".

${ }^{\dagger}$ R.S.Nair and K.Gupta contributed equally to this work.
} 
$[12,25,68,73]$ that has become a key focus in the field of spintronics $[76,77]$ as a promising candidate to generate and manipulate spin currents efficiently [78, 114]. Because the coupling parameter $\xi$ in the SOC term of the Hamiltonian, $\xi$ l.s, scales as the square of the atomic number $Z[17,115]$, heavy metals such as $5 d$ transition metals are expected to exhibit the strongest SHE [35, 99]. Based on this, Pt [81, 84], W [80] and Ta [30] have been studied extensively since they promise large charge-to-spin conversion efficiency that is expressed in terms of the spin Hall angle (SHA) $\Theta_{\mathrm{SH}}[76,77]$. The inverse of the SHE (ISHE) whereby a pure spin current injected into a material gives rise to a transverse charge current, Fig. 3.1(b), is described by the same parameter $\Theta_{\mathrm{sH}}$ and is the principal means by which spin currents are detected $[74,75]$.
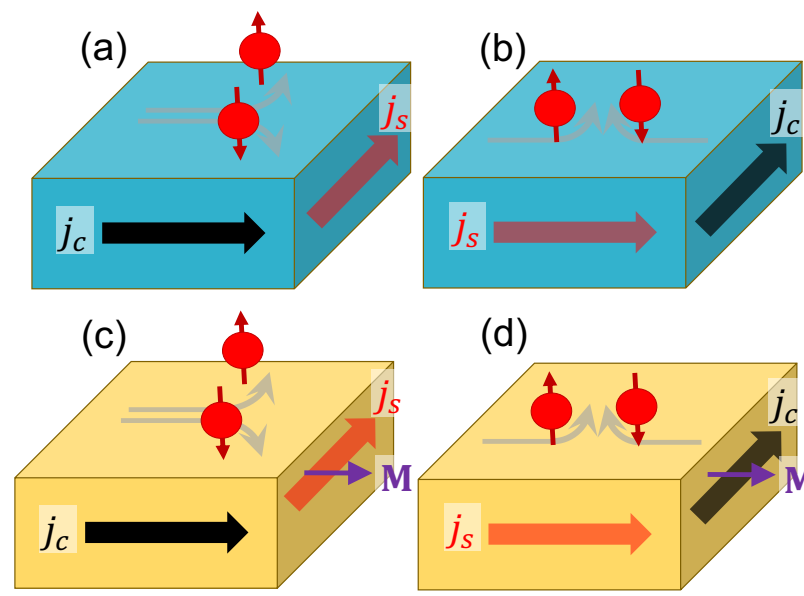

(d)
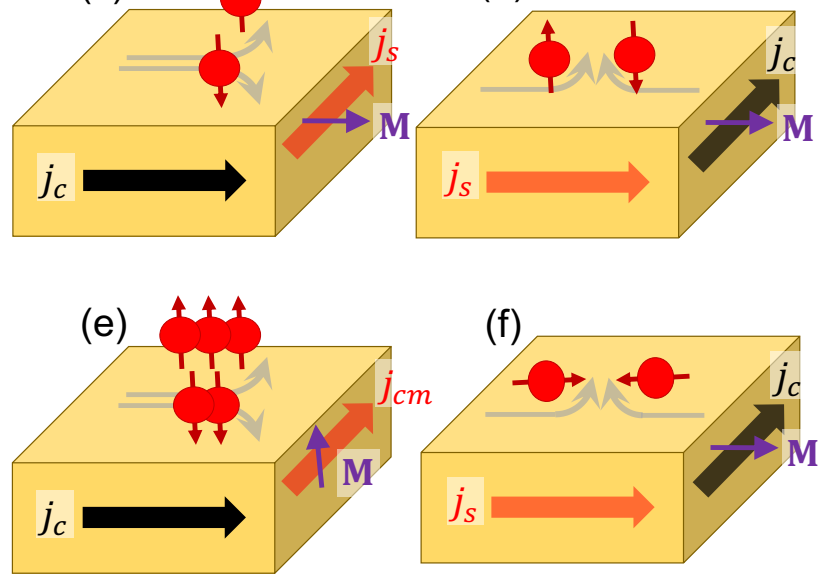

Figure 3.1: (a) SHE and (b) ISHE in a nonmagnetic material, (c) SHE and (d) ISHE in a ferromagnetic material. (e) AHE and (f) IAHE in a ferromagnetic material.

Long before the discovery of the SHE, it was known that in the absence of an external magnetic field, a charge current passed through a ferromagnetic material gives rise to an "anomalous" Hall effect (AHE) when the charge current is not collinear with the magnetization [43], Fig. 3.1(e). Because the response to the charge current is another charge current, the AHE is its own inverse. It therefore only became meaningful to talk about a distinct inverse AHE when it became possi- 
ble to produce and detect pure spin currents, either by "spin pumping" [116-118] and its inverse, by means of the SHE and ISHE, or using the spin Seebeck effect $[119,120]$. In a ferromagnet, the number of spin-up and spin-down conduction channels at the Fermi energy is unequal so that a charge current acquires a spin polarization that is parallel to the magnetization. From this point of view, the response to the initial charge current is a transverse spin current and it might be expected that the injection of a pure spin current with a polarization parallel to the magnetization of the ferromagnet would produce a transverse charge current, Fig. 3.1(f). We call this the inverse AHE (IAHE) to distinguish it from the ISHE in a ferromagnet when the polarization of the injected spin current is orthogonal to the magnetization of the ferromagnet and the direction of injection, Fig. 3.1(d). The reason for referring to the process sketched in Fig. 3.1(d) as the ISHE is clear when one considers what happens when a charge current is passed through a ferromagnet parallel to the magnetization so that there is no AHE; the transverse spin current is expected to be polarized orthogonal to the current and magnetization directions, Fig. 3.1(c). For a long time it was expected that this process would not be of consequence because of dephasing [121]. When the orientation of the polarization of a spin current injected into a ferromagnet is arbitrary with respect to the magnetization direction, no distinction can be made between the ISHE and IAHE.

Miao et al. realized the IAHE (but termed it the ISHE) by using a thermal gradient in the ferromagnetic insulator YIG (Yttrium Iron Garnet) to inject a spin current into the ferromagnetic alloy permalloy $\left(\mathrm{Py}=\mathrm{Ni}_{80} \mathrm{Fe}_{20}\right)$ and produced a transverse charge current with an efficiency comparable to, albeit smaller than, that of Pt [122]. Subsequent experiments [36, 39, 123-135] and theoretical investigations [38, 42, 121, 136, 137] confirm their observation that for Py and other $3 d$ ferromagnetic materials spin Hall effects are comparable in magnitude to those observed in $5 d$ materials leaving one to wonder if the absolute value of the SOC parameter $\xi$ alone determines the size of $\Theta_{\mathrm{sH}}$. The hopping between orbitals on neighbouring atoms that is measured by the bandwidth $W$ reduces the effective spin orbit splitting by mixing the split states [138, 139]. In Fig. 3.2 we plot the ratio of the SOC strength $\xi_{d}$ relative to the bandwidth $W_{d}$ for the valence $d$ states for all transition metals calculated using a tight-binding LMTO code [66] $]^{\ddagger} \mathrm{Ni}$ and Fe have an effective SOC given by the ratio $\xi_{d} / W_{d}$ comparable to that of $5 d$ metals. The relative ease of realizing their stable alloys [140] at a wide range of temperatures makes it worthwhile answering the question: Can large intrinsic spin Hall currents be generated in the Fe-Ni systems?

Understanding SHE in FMs is made complex compared to NMs by the exchange field associated with the magnetization $\mathbf{M}$ of the FM. The curious reader may ask: What is the role of $\mathbf{M}$ in SHE? In order to answer the obvious questions we

\footnotetext{
"We use Mark van Schilfgaarde's "Im" extension of the Stuttgart LMTO (linear muffin tin orbital) code that includes spin-orbit coupling and is maintained in the Queestal suite at https://www.questaal.org.
} 


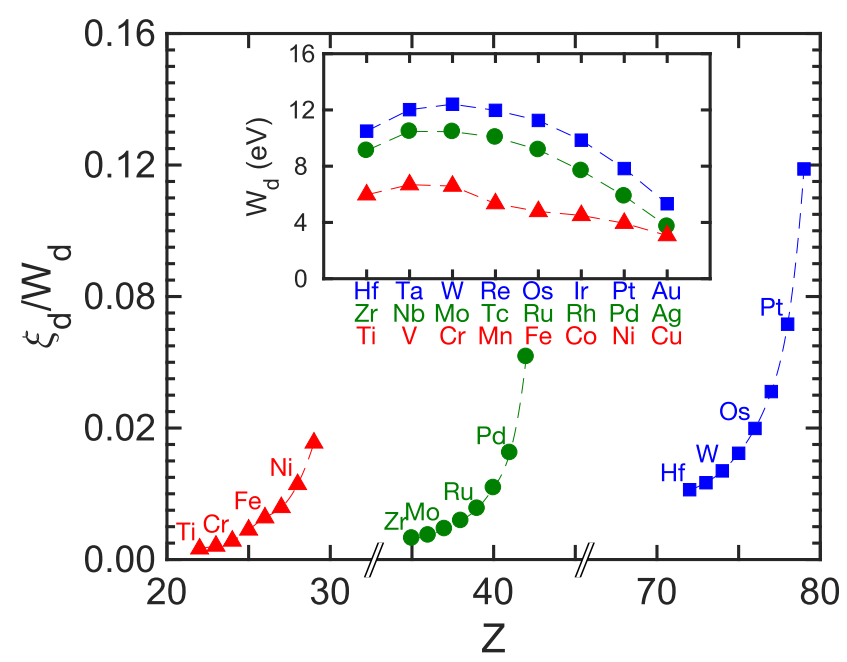

Figure 3.2: A comparison of the spin-orbit coupling parameter $\xi_{d}$ weighted by the $d$-bandwidth $W_{d}$ to give an "effective SOC" for all transition metal elements calculated using a TB-LMTO scheme with atomic sphere approximation(ASA) as a function of the atomic number Z. The bandwidth is given by $\sim 25 \Delta_{d}$ [95], the bandwidth operator in the LMTO-ASA scheme. Inset shows the bandwidth, $W_{d}$ for all transition metal elements.

devise a three-fold approach in this work- (i) We first sketch an intuitive framework based on elastic scattering suitably adapted for a magnetic medium to identify different spin current components excited by a charge current, (ii) we quantify the transverse spin current components in bulk fcc $\mathrm{Ni}_{x} \mathrm{Fe}_{1-x}$ alloys by varying the stoichiometry $x$ as well in bulk fcc Ni and bcc Fe first by setting magnetization along the direction of the driving charge current and (iii) we finally sweep the magnetization in a plane perpendicular to the driving charge current to identify the coupling of the anomalous Hall and spin Hall effects and to extract the angular dependence of all the generated current components in $\mathrm{Ni}, \mathrm{Py}$ and Fe.

\subsection{Mott scattering}

The microscopic mechanism of left-right asymmetry in spin scattering by a spinorbit potential was first worked out by Mott and Massey [27] for the scattering of electron beams. In complex multi-band solids like transition metals there are multiple contributions [77, 141-143] to the spin asymmetry of scattering. However, for a simplified qualitative analysis with a view to identifying different components $\alpha$ of spin current $\mathbf{j}_{s \alpha}$ generated by the scattering of a charge current driven through a ferromagnetic metal, we will adhere to Mott scattering [144, 145] which 
gives the so-called skew scattering contribution [25]. The magnetization direction $\hat{\mathbf{m}}=\mathbf{M} / M$ in a FM determines the spin quantization axis leading to a finite expectation value for the Pauli spin operator $\sigma$. This can be thought of as a finite spin polarization $\mathbf{s}=\langle\boldsymbol{\sigma}\rangle=\boldsymbol{s} \hat{\mathbf{m}}$ of the conduction electrons in the unperturbed system, see Sec. 3.5.1. A perturbing electric field accelerates the conduction electrons in the $z$ direction. These are scattered by a (defect) atom with spin-orbit coupling in the direction $\hat{\mathbf{n}}$ in the $x y$ plane. According to Eq. 3.78 of Ref. [144] the spin polarization of the transverse current is

$$
\mathbf{s}^{\prime}=\left(\alpha+s \hat{\mathbf{m}} . \hat{\mathbf{n}}^{\prime}\right) \hat{\mathbf{n}}^{\prime}+\beta \hat{\mathbf{n}}^{\prime} \times\left(s \hat{\mathbf{m}} \times \hat{\mathbf{n}}^{\prime}\right)+\gamma \hat{\mathbf{n}}^{\prime} \times s \hat{\mathbf{m}}
$$

where $\hat{\mathbf{n}}^{\prime}=\hat{\mathbf{z}} \times \hat{\mathbf{n}}$ is the normal to the scattering plane, see Fig. 3.3. In an electron beam scattering experiment, the coefficients $\alpha, \beta$ and $\gamma$ are functions of scattering amplitudes that transform an initial state into a scattered state [144]. In the semiclassical picture of transport in a metal, these coefficients translate into conversion efficiencies such as the spin Hall angle. For example, for a charge current driven into a NM, for which $s=0$, only the first term $\alpha \hat{\mathbf{n}}^{\prime}$ corresponding to the spin Hall effect survives. Equation (3.1) is also valid for the scattering of a spin polarized current prepared by passing a charge current through a FM and injecting it into a NM. To complete the picture in a ferromagnet, we need to take into account the interaction of the scattered spins with the local magnetization $\mathbf{M}$. This exerts a torque on $\mathbf{s}^{\prime}$ leading to the following equation of motion for the spin polarization $[146]^{\S}$

$$
\frac{\partial \mathbf{s}^{\prime}}{\partial t}+\frac{\partial \mathbf{j}_{s^{\prime}}}{\partial n}+\frac{\mathbf{s}^{\prime} \times \mathbf{M}}{\tau_{\mathrm{pr}}}=-\frac{\mathbf{s}^{\prime}}{\tau_{\mathrm{sf}}}
$$

where $\mathbf{j}_{s^{\prime}}^{\hat{\mathbf{n}}}$ is the local spin current, polarized in the $\mathbf{j}_{\mathbf{s}^{\prime}} / j_{s^{\prime}}$ direction and propagating in the direction $\hat{\mathbf{n}}$. $\tau_{\mathrm{pr}}$ and $\tau_{\mathrm{sf}}$ are the spin precession and spin-flip relaxation times, respectively. In a steady state we can set $\frac{\partial \mathbf{s}^{\prime}}{\partial t}=0$, and in a relaxation time approximation the average spin current in the direction $\hat{\mathbf{n}}$ is

$$
\mathbf{j}_{s^{\prime}}^{\hat{\mathbf{n}}}=\lim _{L \rightarrow \infty} \frac{1}{2 L} \int_{-L}^{L}\left(\frac{\mathbf{M} \times \mathbf{s}^{\prime}}{\tau_{\mathrm{pr}}}-\frac{\mathbf{s}^{\prime}}{\tau_{\mathrm{sf}}}\right) d n
$$

We consider the case of a homogeneous ferromagnet such that $\mathbf{M}$ and the scattering are constant in space, then (3.3) reduces to

$$
\mathbf{j}_{s^{\prime}}^{\hat{\mathbf{n}}}=\frac{\mathbf{M} \times \mathbf{s}^{\prime}}{\tau_{\mathrm{pr}}}-\frac{\mathbf{s}^{\prime}}{\tau_{\mathrm{sf}}}
$$

Using (3.1) in (3.4), we can obtain all components of intrinsically generated spin currents in a FM. The first term on the right-hand side (rhs) in (3.4) corresponds

\footnotetext{
${ }^{\S}$ We omit the dephasing term $\propto 1 / \tau_{\mathrm{dp}}$ since any detectable transverse spin states would be eigenstates of the perturbed system and therefore should not de-phase.
} 


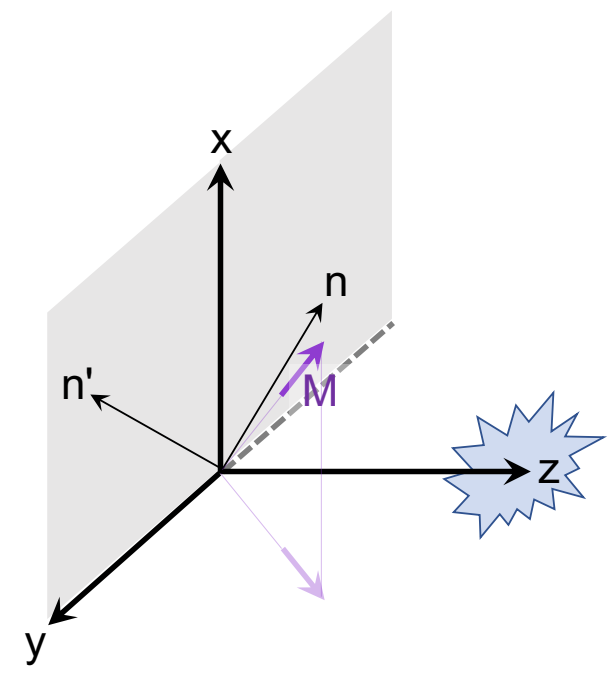

Figure 3.3: A schematic showing the generation of a transverse spin current in the $x y$ plane in a magnetic material when a current flows in the $z$ direction. The magnetization $\mathbf{M}$ of the medium (purple arrow) determines the spin quantization axis. Upon elastic scattering mediated by spin-orbit coupling, spins are asymmetrically (skew) scattered leading to a transverse spin polarization. The magnetization of the medium interacts with the scattered spin leading to finite rotation of the spin orientation.

to the precession of the transverse spin components of $\mathbf{s}^{\prime}$ about the magnetization of the FM on a length scale $l_{\text {pr }}$ corresponding to the familiar Larmor precession while the second term describes spin flip scattering on a length scale of $l_{\mathrm{sf}}$ and one should possibly distinguish between components of $\mathbf{s}^{\prime}$ parallel and perpendicular to $\mathbf{M}$. These expressions will not be used to make quantitative estimates but just to qualitatively understand the results of our first principles calculations.

To simplify matters, we look at the case where the magnetization is parallel to the applied driving charge current (or electric field) in the $z$ direction i.e, $\mathbf{M} \| \hat{\mathbf{z}}$ and $\hat{\mathbf{n}}$ is in the $x y$ plane. From (3.4), we obtain with $\hat{\mathbf{n}}=x, y$

$$
\begin{aligned}
& \mathbf{j}_{s^{\prime}}^{x}=\left(\frac{M \gamma^{\prime}}{\tau_{\mathrm{pr}}}-\frac{\alpha}{\tau_{\mathrm{sf}}}\right) \hat{\mathbf{y}}-\left(\frac{\gamma^{\prime}}{\tau_{\mathrm{sf}}}+\frac{M \alpha}{\tau_{\mathrm{pr}}}\right) \hat{\mathbf{x}}-\frac{\beta^{\prime}}{\tau_{\mathrm{sf}}} \hat{\mathbf{z}} \\
& \mathbf{j}_{\mathrm{s}^{\prime}}^{y}=\left(\frac{\alpha}{\tau_{\mathrm{sf}}}-\frac{M \gamma^{\prime}}{\tau_{\mathrm{pr}}}\right) \hat{\mathbf{x}}-\left(\frac{\gamma^{\prime}}{\tau_{\mathrm{sf}}}+\frac{M \alpha}{\tau_{\mathrm{pr}}}\right) \hat{\mathbf{y}}-\frac{\beta^{\prime}}{\tau_{\mathrm{sf}}} \hat{\mathbf{z}}
\end{aligned}
$$

where $\left\{\beta^{\prime}, \gamma^{\prime}\right\}=\{s \beta, s \gamma\}$. The first component in (3.5a) and (3.5b) has the symmetry of a regular spin Hall effect in a NM and results from a competition between two different relaxation mechanisms in a FM. The second component, which is the new spin swapping effect (SSE), preserves the sign under rotation of axes $x \rightarrow y$. 
The third term is such that the spins align with the magnetization by spin flip scattering via a conventional Elliott-Yafet (EY) mechanism [23, 24]. While the SHE and EY terms are well studied, SSE has not gained much traction although such a current component appears even within the first Born approximation in scattering theory unlike the SHE [40]. In the following section we will examine the intrinsic SSE and show that it can be quite significant ?

\subsection{Calculations}

The quantum mechanical scattering problem [58] was solved for a scattering region comprising disordered bulk material embedded between lattice matched ballistic leads. The $\mathrm{Cu}$ lead potential as well as $\mathrm{Ni}$ and Fe potentials for the scattering region were calculated in the atomic spheres (AS) approximation (ASA) with a tightbinding (TB) muffin-tin orbital (MTO) basis [67, 95, 147]. For fcc $\mathrm{Ni}_{x} \mathrm{Fe}_{1-x}$ substitutional random alloys, AS potentials for $\mathrm{Ni}$ and Fe were evaluated self-consistently using the coherent potential approximation (CPA) [148] implemented with TBMTOs [149] using the experimental lattice constants $a_{x}$ [140] and spd basis. The resulting $\mathrm{Ni}$ and $\mathrm{Fe}$ AS potentials were used to randomly populate fcc lattice sites corresponding to the stoichiometry $x$ in lateral supercells transverse to the [001] transport direction. For RT calculations, thermal disorder was introduced by displacing atoms from their ideal lattice positions with a Gaussian distribution characterized by the root mean square displacement $\Delta_{r}^{T}$ with $T=300 \mathrm{~K}$. Spin disorder with respect to the $T=0$ collinear magnetization direction was described analogously with a Gaussian distribution of polar rotation angles $\theta$ characterized by the root mean square rotation $\Delta_{\theta}^{T}$ together with a uniform distribution in the azimuthal angle $\phi[57,61,150] . \Delta_{\theta}^{T}$ was chosen to reproduce the experimentally observed room temperature magnetization $[140,151,152]$. With $\Delta_{\theta}^{T}$ fixed, $\Delta_{r}^{T}$ was chosen to reproduce the experimental room temperature resistivity $\rho_{x}$.

All of the computational results to be presented below were obtained with scattering geometries constructed using lateral supercells of $6 \times 6$ atoms repeated periodically in the $x y$ plane with 300 such atomic layers stacked in the $z$ direction. For the fcc alloys including pure $\mathrm{Ni}$ as well as for bcc Fe, the $z$ axis is set to be in the [001] direction. A BZ grid with a sampling density equivalent to $174 \times 174 \mathrm{k}$ points for a $1 \times 1$ unit cell was used in all cases. Transverse spin current generation is studied by injecting a charge current in the $z$ direction from ideal ballistic leads. Lattice matched fcc $\mathrm{Cu}$ leads were used for the alloys and $\mathrm{Ni}$, while Fe calculations were performed using bcc Fe leads.

\footnotetext{
'In the context of the spin-polarization of a charge current in a ferromagnet, the term swapping could be misleading as it was proposed originally for the exchange of spin and momentum directions of a polarized current injected into a non-magnetic material [40] upon scattering by SOC. Since both terms have the same symmetry, we refer to the new term in Eq. (3.5) as SSE to avoid a surfeit of terminology.
} 
In the scattering calculation, the full quantum mechanical wavefunction is obtained using a wave-function matching scheme [56] implemented [16, 94] with a TB-MTO basis and generalized to include spin-orbit coupling and noncollinearity $[57,153]$. The wave function obtained for the scattering region is used to determine position dependent charge and spin currents [20] expressed as the current tensor $\left(j_{c}^{\beta}, j_{s x}^{\beta}, j_{s y}^{\beta}, j_{s z}^{\beta}\right)$ where $\beta=x, y, z$ is the direction of current flow. In the following, we present the transverse spin currents $j_{s x}^{x}, j_{s x}^{y}, j_{s y}^{x}, j_{s y}^{y}$ and charge currents $j_{c}^{x}, j_{c}^{y}$ that we calculate in response to a charge current $j_{c}^{z}$ injected along the $z$ direction. The results that we will show were determined far from the leads to avoid any interface effects and are characteristic of the bulk. The spin Hall angle is estimated as $\Theta_{s \mathrm{H}}=j_{s y}^{x} / j_{c}^{z}\left(=-j_{s x}^{y} / j_{c}^{z}\right)$. For bulk non-magnetic materials, $j_{s y}^{x}$ and $j_{s x}^{y}$ are equal in magnitude $[20,34]$. The spin swapping angle is defined as $\Theta_{\mathrm{ss}}=j_{s y}^{y} / j_{c}^{z}$ $\left(=j_{s x}^{x} / j_{c}^{z}\right)$. The anomalous Hall angle is the normalized transverse charge current given by $\Theta_{\mathrm{aH}}=j_{c}^{x} / j_{c}^{z}$ when the magnetization is in the $x$ direction or equivalently $j_{c}^{y} / j_{c}^{z}$ when the magnetization is in the $y$ direction.

\subsubsection{Magnetization parallel to the driving current}

When $\mathbf{M} \| \mathbf{j}_{c}^{z}$, there is no AHE and transverse spin currents $j_{s x}^{y}$ and $j_{s y}^{x}$ are generated with equal magnitude and opposite signs, just as in the case of nonmagnetic $\mathrm{Pt}$ $[20,34]$ and in agreement with the first term in (3.5). The values of $\Theta_{\mathrm{sH}}$ obtained for bcc Fe, fcc $\mathrm{Ni}$ and the fcc substitutional $\mathrm{Ni}_{x} \mathrm{Fe}_{1-x}$ alloys for $0.4<x<0.95$ [140] are plotted as a function of concentration $x$ in Fig. 3.4(a). $\Theta_{\mathrm{sH}}$ increases from $\sim 1 \%$ in bcc Fe to $\sim 7.5 \%$ in fcc $\mathrm{Ni}$ at room temperature. For the alloys, calculations are performed with and without thermal disorder and temperature appears to play a very minor role. The values of $\Theta_{\mathrm{sH}}$ calculated for $0 \mathrm{~K}$ and $300 \mathrm{~K}$ are approximately equal indicating that scattering by alloy disorder dominates scattering by phonons. The effect of temperature becomes significant only in the weak alloy regime $(x \geq$ 0.9). The value of $\Theta_{\mathrm{sH}}^{\mathrm{Py}} \sim 4.5 \%$ that we find for Py $\left(\mathrm{Ni}_{0.8} \mathrm{Fe}_{0.2}\right)$, is very close to the value we calculate for bulk Pt at $300 \mathrm{~K}[20,34]$. As a function of $x$, we find that $\Theta_{\mathrm{sH}}$ scales roughly as $x \sigma_{\mathrm{sH}}^{\mathrm{Ni}}+(1-x) \sigma_{\mathrm{sH}}^{\mathrm{Fe}}$ where $\sigma_{\mathrm{sH}}^{\mathrm{Ni}(\mathrm{Fe})} \equiv \Theta_{\mathrm{sH}}^{\mathrm{Ni}(\mathrm{Fe})} / \rho^{\mathrm{Ni}(\mathrm{Fe})}$ is the spin Hall conductivity at RT. This remarkably simple relationship is consistent with the finding that the Fe and Ni CPA AS potentials calculated for the alloys are essentially independent of the stoichiometry $x$, see Appendix 3.5.3; the effect of alloying can in this sense be reduced to purely one of concentration.

Fig. 3.4(b) presents first quantitative estimates of the spin swapping angle $\Theta_{\mathrm{ss}}$ in $\mathrm{Ni}_{x} \mathrm{Fe}_{1-x}$ alloys. For all values of $x$ studied, as well as for bcc Fe, the spin swapping currents $j_{s x}^{x}$ and $j_{s y}^{y}$ are equal in magnitude and sign in agreement with the prediction of equation (3.5). $\Theta_{\mathrm{ss}}$ ranges from $1.5 \%$ in bcc Fe to $-2.5 \%$ in fcc $\mathrm{Ni}$ at room temperature. Unlike $\Theta_{\mathrm{sH}}, \Theta_{\mathrm{ss}}$ is found to be weakly dependent on $x$ for $x<0.8$ but after switching sign for a concentration close to that of Py, it increases rapidly in magnitude to $-2.5 \%$ in $\mathrm{Ni}$. From the Mott scattering analysis, it is apparent 


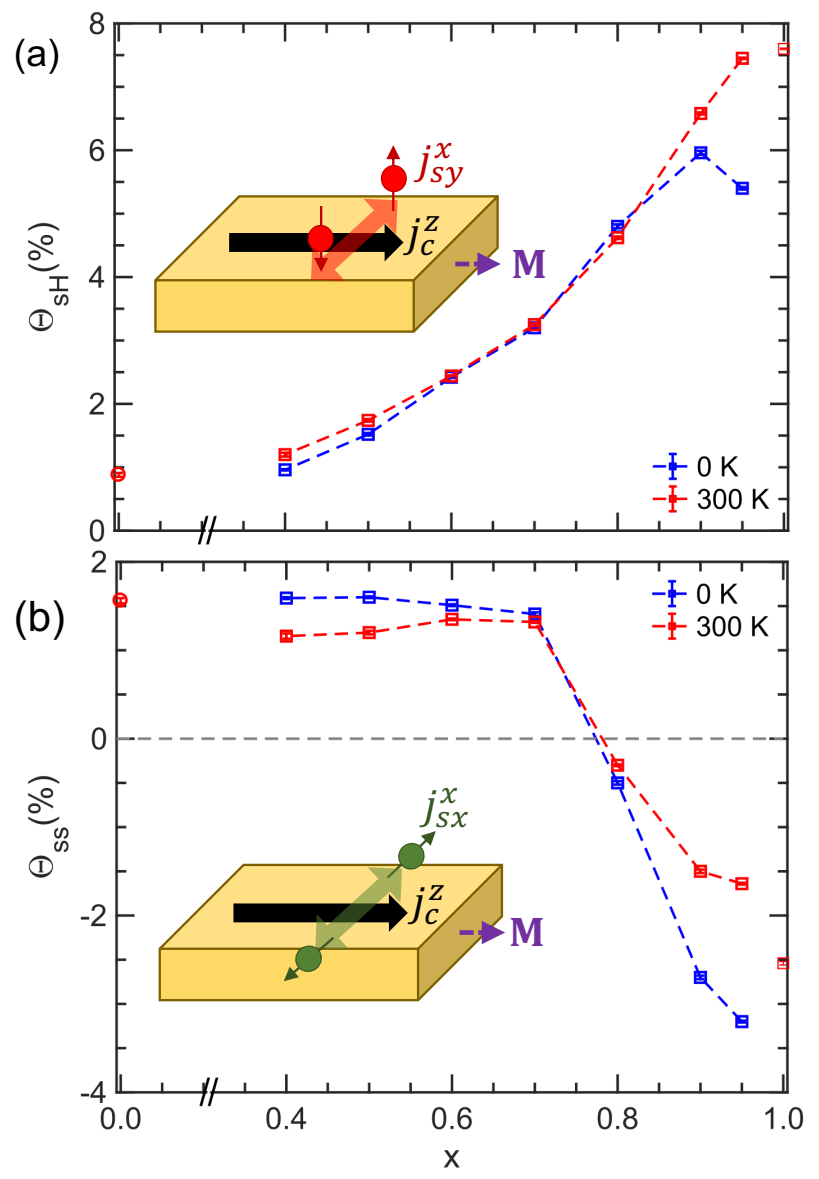

Figure 3.4: Spin Hall angle $\Theta_{\mathrm{sH}}$ (a) and spin swapping angle $\Theta_{\mathrm{ss}}$ (b) calculated as a function of alloy concentration $x$ in $\mathrm{Ni}_{x} \mathrm{Fe}_{1-x}$ with only alloy disorder at $0 \mathrm{~K}$ (blue) and with room temperature lattice and spin disorder (red). Calculations were performed for bcc Fe (circle) and fcc $\mathrm{Ni}_{x} \mathrm{Fe}_{1-x}$ up to $\mathrm{Ni}$ (squares) with experimentally determined lattice constants. The alloy calculations are for concentrations for which the fcc phase is stable $(x \geq 0.4)$. Insets: schematics of asymmetric spin scattering. With SOC included, a charge current $j_{c}^{z}$ (black arrow) injected into a magnetic material parallel to the magnetization $\hat{\mathbf{m}}$ (purple arrow) in the $z$ direction leads to spin currents in the transverse direction (red and green arrows) with the spin directions indicated by the spheres with arrows.

that the magnitude and sign of different components of transverse spin currents are determined by the interplay between scattering probability amplitudes that are governed by details of the electronic structure and disorder, and the relaxation mechanisms: spin precession $\propto 1 / \tau_{\mathrm{pr}}$ and momentum relaxation $\propto 1 / \tau_{\mathrm{sf}}$. There 
is considerable scope for a deeper theoretical study to disentangle these effects quantitatively which however will not be attempted here.

\section{Origin of the large SHE in Ni}

The above results establish that the spin Hall effect in $3 d$ materials is comparable in magnitude to that found in Pt $[20,93]$ and shows a very weak temperature dependence for the fcc $\mathrm{Ni}_{x} \mathrm{Fe}_{1-x}$ alloys. The large size of $\Theta_{\mathrm{sH}}$ in Pt, $\mathrm{W}$ and Ta is usually attributed to the size of the SOC in the $5 d$ elements $[35,99]$. Our results suggests that all else being equal, the "effective SOC" that we defined as $\xi_{d} / W_{d}$ in the introduction is a more appropriate measure for comparing SOC related effects across the $3 d, 4 d$ and $5 d$ series than the bare value of $\xi_{d}$. Other band structure effects can have a much larger impact than the size of the SOC. In chapter 2, it has been shown that the magnitude as well as the sign of $\Theta_{\mathrm{sH}}$ depends on the filling of the outer $d$ shell $[20,35,99]$, lattice structure and proximity of degeneracies at high symmetry points to the Fermi-level in $5 d$ metals.

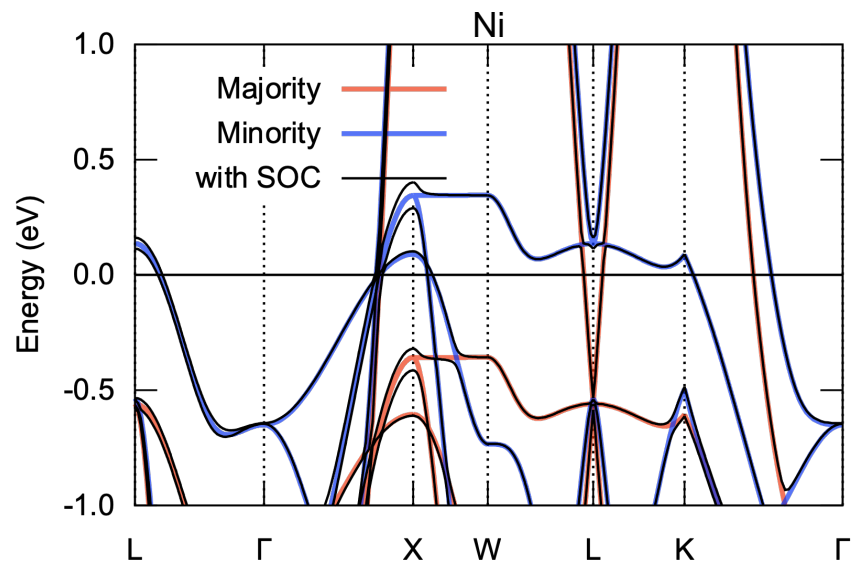

Figure 3.5: Majority (red) and minority(blue) spin bands for Ni calculated without SOC. Spin mixed bands (black) for Ni calculated with SOC. The horizontal line through zero represents Fermi level.

To study the role played by the band structure in giving rise to a very large value of $\Theta_{\mathrm{sH}}^{\mathrm{Ni}}$, we compare the band structures of Ni calculated self consistently using a TB-LMTO-ASA code ${ }^{\S}$ with and without SOC in Fig. 3.5. The features that attract our attention are the uppermost $d$ bands corresponding to the unoccupied minority (blue) and occupied majority (red) spin bands that are nearly flat in a large volume of the Brillouin zone (BZ) from X to $\mathrm{W}$ and from halfway between $\mathrm{W}$

\footnotetext{
${ }^{\S}$ We use Mark van Schilfgaarde's "lm" extension of the Stuttgart LMTO code that treats noncollinear magnetization and spin-orbit coupling and is maintained in the QUESTAAL suite at https://www.questaal.org.
} 
and $\mathrm{L}$ to $\mathrm{L}$ to $\mathrm{K}$ in Fig. 3.5 as well as the corresponding two-fold degenerate states at $\mathrm{X}$ and $\mathrm{L}$. We immediately see that the double degeneracies at $\mathrm{X}$ and $\mathrm{L}$ are lifted by SOC with splittings of $\sim 0.10 \mathrm{eV}$ and $\sim 0.05 \mathrm{eV}$ respectively. By analogy with Pt [99], we attribute the large $\Theta_{\mathrm{sH}}$ in Ni compared to Fe and Co in experiments $[39,154]$ to the proximity of the Fermi level to these quasi-degenerate states that are strongly affected by SOC.

\subsubsection{Magnetization perpendicular to the driving current}

One important consequence of SOC in ferromagnets is the anomalous Hall effect (AHE) [43]; when a current is passed through a ferromagnet in the absence of an external magnetic field, a Hall effect $\propto \mathbf{j}_{c}^{z} \times \hat{\mathbf{m}}$ is observed transverse to the charge current and the magnetization. So far, we have studied the SHE and SSE for $\hat{\mathbf{m}} \| \hat{\mathbf{z}}$ so that the AHE vanishes. In this section, we study the spin and charge current response in the $x y$ plane perpendicular to the charge current direction when the magnetization is also in the $x y$ plane, $\hat{\mathbf{m}} \perp \hat{\mathbf{z}}$. By doing so, we will be able to observe how the two depend on the magnetization and how they are related.

A charge current $j_{c}^{z}$ is injected in the $z$ direction. The magnetization is oriented in the $x y$ plane at an angle $\phi$ to the $x$ axis that is varied from $-\pi$ to $\pi$. We calculate the following transverse responses: $\Theta_{\mathrm{aH}}=j_{c}^{x} / j_{c}^{z}, \Theta_{\mathrm{sH}}=j_{s y}^{x} / j_{c}^{z}$ and $\Theta_{\mathrm{ss}}=j_{s x}^{x} / j_{c}^{z}$ describing the anomalous Hall, spin Hall and spin swapping effects respectively and plot the results for Ni at $300 \mathrm{~K}$ in Fig. 3.6. The three effects exhibit quite distinct behaviour and similar results are found for Py and Fe. The functional dependence of the response at $300 \mathrm{~K}$ is given for all three materials in Table 3.1. The functional form of the AHE is given by the cross product of the injected charge current $\mathbf{j}_{c}^{z}$ and the magnetization $\hat{\mathbf{m}}=\cos \phi \hat{\mathbf{x}}+\sin \phi \hat{\mathbf{y}}$. The transverse charge response is thus: $A_{\mathrm{aH}} j_{c}^{z}[\hat{\mathbf{z}} \times(\cos \phi \hat{\mathbf{x}}+\sin \phi \hat{\mathbf{y}})]=A_{\mathrm{aH}} j_{c}^{z}(\cos \phi \hat{\mathbf{y}}-\sin \phi \hat{\mathbf{x}})$. By fitting $\Theta_{\mathrm{aH}}=j_{c}^{x} / j_{c}^{z}$ plotted in Fig. 3.6(a), we evaluate the constant $A_{\mathrm{aH}}$ for $\mathrm{Ni}=1.33 \%, \mathrm{Py}=0.35 \%$ and $\mathrm{Fe}=0.46 \%$ as shown in Table 3.1 .

From (3.4) we obtain the following form for the components of the spin current

$$
\begin{aligned}
& j_{s y}^{x}=C_{1}+C_{2} \cos 2 \phi+C_{3} \sin \phi \\
& j_{s x}^{x}=-C_{2} \sin 2 \phi+C_{4} \cos \phi
\end{aligned}
$$

where the tedious algebra leading to (3.6) is outlined in Sec. 3.5.2. We begin with the spin Hall case $\Theta_{\mathrm{sH}}=j_{s y}^{x} / j_{c}^{z}$ and find the best fit to the calculated spin Hall currents is $\Theta_{\mathrm{sH}}(\phi)=C_{\mathrm{sH}}+A_{\mathrm{sH}} \cos 2 \phi+B_{\mathrm{sH}} \cos 4 \phi$ where the two leading terms are the same as in (3.6a) and the coefficient of the $\cos 4 \phi$ term is an order of magnitude smaller. It therefore transpires that the dominant mechanism of bulk spin current generation mediated by alloy disorder or phonons is skew scattering. The higher order discrepancy could result from other mechanisms contributing to the spin Hall 


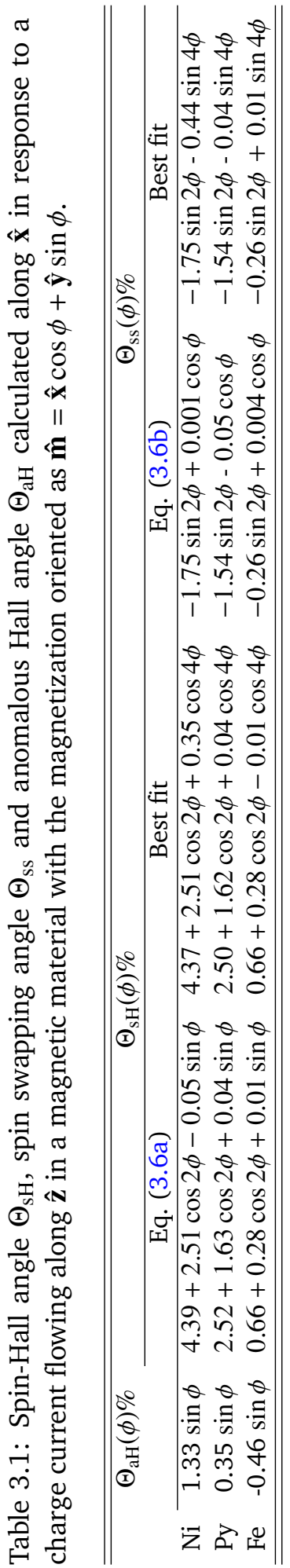



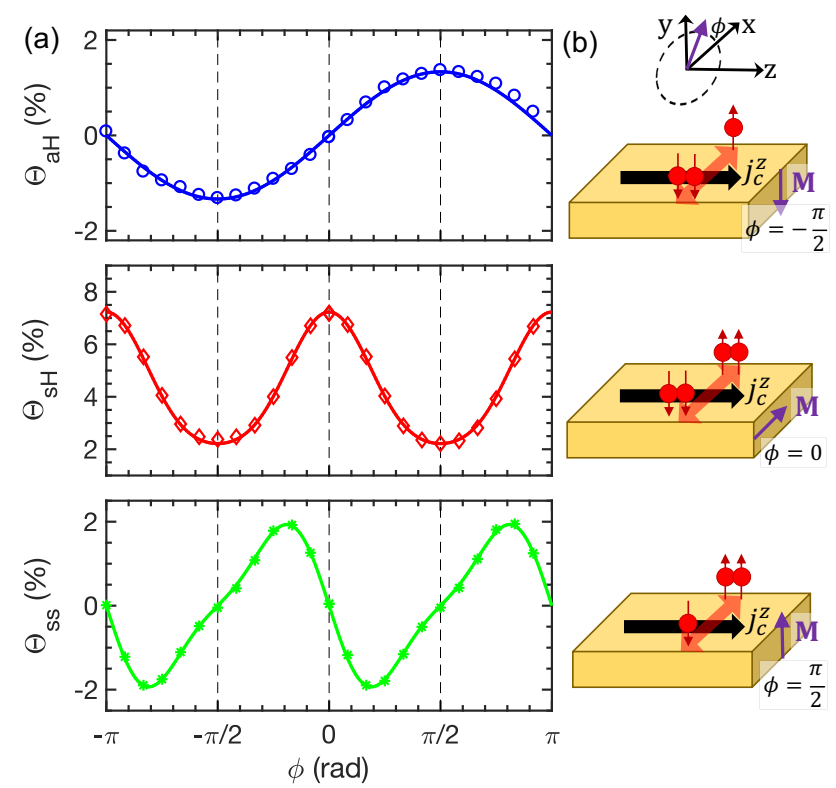

Figure 3.6: (a) Anomalous Hall angle $\Theta_{\mathrm{aH}}$ (blue circles), spin Hall angle $\Theta_{\mathrm{sH}}$ (red diamonds) and spin swapping angle $\Theta_{\mathrm{ss}}$ (green stars) in room temperature Ni generated along the $\hat{\mathbf{x}}$ direction in response to a charge current along $\hat{\mathbf{z}}$. All three are calculated as a function of the magnetization direction in the $x y$ plane described by the angle $\phi$ with respect to $\hat{\mathbf{x}}$. The solid lines are the best fits to the empirical data that are given in Table 3.1. (b) For a charge current $j_{c}$ along $\hat{\mathbf{z}}$, spins oriented along $\hat{\mathbf{y}}$ are scattered in the $\hat{\mathbf{x}}$ direction for the three different magnetization directions $\phi=-\pi / 2,0,-\pi / 2$ (top to bottom).

effect that are not accounted for in Mott scattering. For Ni, $\Theta_{\mathrm{sH}}$ ranges between $2.2 \%$ and $7.3 \%$ and does not change sign. The maximum value is obtained at $\phi=0, \pm \pi$ and is approximately equal to the value extracted for $\hat{\mathbf{m}} \| \hat{\mathbf{z}}$. We also find from (3.19) that (3.6a) reduces to the expression in (3.5a) at $\phi=0, \pm \pi$ in agreement with our calculations. For these angles, $\hat{\mathbf{m}}= \pm \hat{\mathbf{x}}$ is perpendicular to the spin direction $\hat{\mathbf{y}}$. The minimum occurs for $\phi= \pm \pi / 2$ when $\hat{\mathbf{m}}= \pm \hat{\mathbf{y}}$ is parallel to the spin. It seems counter-intuitive that the largest value of $\Theta_{\mathrm{sH}}$ is obtained when the spins generated by the SHE are oriented perpendicular to the magnetization while the SHE is reduced when the spins are parallel to it.

Comparison of the functional forms of the AHE and SHE does not suggest any simple relation between the two effects. Unlike $\Theta_{\mathrm{aH}}, \Theta_{\mathrm{sH}}$ does not change sign. However, if we focus on the magnitudes $\left|\Theta_{\mathrm{sH}}\right|$ and $\left|\Theta_{\mathrm{aH}}\right|$, a relation emerges that we can describe with the help of the sketches in Fig. 3.6(b). First we look at the case $\phi=0$ to revisit the conventional SHE. Up-spin and down-spin electrons are scattered symmetrically in opposite directions giving equal currents $j_{\uparrow y}^{x}$ and $j_{\downarrow y}^{x}$ 
respectively. Here, $j_{\uparrow y}^{x}$ and $j_{\downarrow y}^{x}$ represents the current of spins (not the magnetic moment caused by the spin) pointing parallel and anti-parallel to the $y$ direction flowing in the $x$ direction. Because of their opposite directions, the total spin current $j_{s y}^{x}$ is given by $\left|j_{\uparrow y}^{x}\right|+\left|j_{\downarrow y}^{x}\right|$. The total charge current is $j_{c}^{x}=\left|j_{\uparrow y}^{x}\right|-\left|j_{\downarrow y}^{x}\right|=0$. For $\phi=\pi / 2$, the majority spin-down $d$ bands are fully occupied and it is the minority spin-up electrons that are preferentially scattered. The preferential scattering leads to a finite $j_{c}^{x}$. For $\phi=-\pi / 2$, it is the minority down-spin electrons that are preferentially scattered. This switches the sign of $j_{c}^{x}$, but $j_{s y}^{x}$ given by $\left|j_{\uparrow y}^{x}\right|+\left|j_{\downarrow y}^{x}\right|$ remains unchanged. Therefore, following Zhang [68] and Davidson et al. [42], we propose that the AHE is a special case of the SHE.

The SSE in ferromagnets behaves as $\Theta_{\mathrm{ss}}=A_{\mathrm{sS}} \sin 2 \phi+B_{\mathrm{ss}} \sin 4 \phi$, where the dominant first term in the fit is the same as in (3.6b). For Ni, the maximum and minimum are $\pm 1.93 \%$ obtained at $\pm 0.19 \pi$ respectively. From (3.6a) and (3.6b), the relationship between $\Theta_{\mathrm{sH}}$ and $\Theta_{\mathrm{ss}}$ is the occurrence of the same $C_{2}$ coefficient in the dominant angular dependent term. All coefficients are determined by fitting to the angular dependence of the results of the detailed calculations. For Py the values of $C_{2}$ turn out to be approximately equal, as shown in Table 3.1. For Ni they are seen to be quite comparable in size. The deviations can be attributed to the fact that the detailed calculations are not based upon a particular scattering mechanism like the skew scattering included in Mott scattering. They include other mechanisms whose predictions for $j_{s x}^{x}$ and $j_{s y}^{x}$ may differ from those of skew scattering ${ }^{\dagger}$

\subsection{Discussion}

The inverse spin Hall effect (ISHE) refers to the charge current that is generated transverse to a spin current and its polarization. The ISHE in Co that was reported to result from the injection of a pure spin current by Tian et al.[129] was found to be independent of the magnetization orientation of Co. Based on the spin absorption measurements, a simple relationship $\Theta_{\mathrm{aH}}=\beta \Theta_{\mathrm{sH}}$ was proposed by Omori et al.[39]. We find that the estimated values of the bulk polarization $\beta$ determined from the coefficients of our empirical fit as $A_{\mathrm{aH}} / A_{\mathrm{sH}}$ is at big variance from the explicitly calculated values of $\beta$ in Ni and Py [20]. Recent experiments observed a transverse out of plane spin current generated by a ferromagnet with in-plane magnetization in a magnetic multilayer geometry $[37,155]$. A spin current of the same symmetry has been shown to originate at a FM|NM interface[156]. It is transpar-

'There is an additional normalization to the spin polarization, see Eq. 3.78 in Ref. [144]

$$
s^{\prime} \rightarrow \frac{s^{\prime}}{1+\alpha \mathbf{s} . \hat{\mathbf{n}}}
$$

where $\frac{1}{1+\alpha s . \hat{\mathbf{n}}}=1$ for $\hat{\mathbf{m}} \| \hat{\mathbf{z}}$ while this will introduce higher order terms $O\left(\alpha s^{\prime} s \cos \phi\right)$ to $j_{s x}^{x}$ and $j_{s y}^{x}$ for $\hat{\mathbf{m}} \perp \hat{\mathbf{z}}$. 
ent from the present study that a detectable signal of the same spin current could originate from the bulk itself by spin swapping effect.

There has been a handful of theoretical studies [38, 41, 42, 157] since the experimental observation of SHE in FMs to unravel the mechanisms behind nontrivial transverse spin current generation in a FM which was otherwise believed to be killed by the exchange field of the FM. These studies have revealed essential ingredients and underlying symmetries for spin current generation in a FM. By treating electronic structure and disorder on equal footings, we presented quantitative estimates for spin Hall $\Theta_{\mathrm{sH}}$, spin swapping $\Theta_{\mathrm{ss}}$ and anomalous Hall $\Theta_{\mathrm{aH}}$ angles in bulk $\mathrm{Ni}_{x} \mathrm{Fe}_{1-x}$ systems as a function of $x$ and demonstrated the magnetization dependence of these effects entirely from first principles at room temperature as well as at $0 \mathrm{~K}$ without any adjustable parameters. We presented an intuitive picture of transverse spin current generation in a FM using Mott scattering. $3 d$ FMs should be undisputedly researched as candidate materials for spintronic applications especially as they offer possibilities for switching of perpendicular magnetization by intrinsically generated spin currents that can be predictably controlled using the magnetization of the injecting FM as revealed in this work.

\subsection{Appendices}

\subsubsection{Relationship between $\mathrm{s}$ and $\hat{\mathbf{m}}$}

We start with a magnetization vector $\mathbf{M}$ pointing in an arbitrary direction specified by the polar angles $(\theta, \phi)$ so that

$$
\mathbf{M}=M \sin \theta \cos \phi \hat{\mathbf{x}}+M \sin \theta \sin \phi \hat{\mathbf{y}}+M \cos \theta \hat{\mathbf{z}}
$$

It is natural to assume the spin quantization axis of the system to lie along the unit vector $\hat{\mathbf{m}}=\mathbf{M} / M$ in terms of which the spin operator $\sigma_{m} \equiv \boldsymbol{\sigma} \cdot \hat{\mathbf{m}}$ is

$$
\boldsymbol{\sigma} . \hat{\mathbf{m}}=\left(\begin{array}{cc}
\cos \theta & \sin \theta e^{-i \phi} \\
\sin \theta e^{i \phi} & -\cos \theta
\end{array}\right)
$$

where $\boldsymbol{\sigma}$ is a vector of Pauli spin matrices. A general normalized spin state $\chi=\left(\begin{array}{l}a \\ b\end{array}\right)$ is defined with $a^{2}+b^{2}=1$. On solving $\sigma_{m} \chi=+\chi$, we obtain the up-spin state

$$
\chi_{+}=\left(\begin{array}{c}
\cos \frac{\theta}{2} \\
\sin \frac{\theta}{2} e^{i \phi}
\end{array}\right)
$$


The spin polarization $\mathbf{s}=\boldsymbol{s} \hat{\mathbf{m}}$ of a medium defined as the expectation value of the Pauli spin operator $\boldsymbol{\sigma}$ is

$$
\begin{array}{r}
\left\langle\chi_{+}|\boldsymbol{\sigma}| \chi_{+}\right\rangle=\left(\cos \frac{\theta}{2} \sin \frac{\theta}{2} e^{-i \phi}\right)\left(\begin{array}{cc}
0 & 1 \\
1 & 0
\end{array}\right)\left(\begin{array}{c}
\cos \frac{\theta}{2} \\
\sin \frac{\theta}{2} e^{i \phi}
\end{array}\right) \hat{\mathbf{x}} \\
+\left(\cos \frac{\theta}{2} \sin \frac{\theta}{2} e^{-i \phi}\right)\left(\begin{array}{cc}
0 & -i \\
i & 0
\end{array}\right)\left(\begin{array}{c}
\cos \frac{\theta}{2} \\
\sin \frac{\theta}{2} e^{i \phi}
\end{array}\right) \hat{\mathbf{y}} \\
+\left(\cos \frac{\theta}{2} \sin \frac{\theta}{2} e^{-i \phi}\right)\left(\begin{array}{cc}
1 & 0 \\
0 & -1
\end{array}\right)\left(\begin{array}{c}
\cos \frac{\theta}{2} \\
\sin \frac{\theta}{2} e^{i \phi}
\end{array}\right) \hat{\mathbf{z}} \\
=\sin \theta \cos \phi \hat{\mathbf{x}}+\sin \theta \sin \phi \hat{\mathbf{y}}+\cos \theta \hat{\mathbf{z}} \equiv \hat{\mathbf{m}}
\end{array}
$$

Here $s=1$ because we started with the system in the normalized spin-up state $\chi_{+}$. In a metallic solid, the appropriate generalization results in the density-of-states (DoS) polarisation

$$
s=\frac{\psi_{\uparrow}^{2}\left(\varepsilon_{F}\right)-\psi_{\downarrow}^{2}\left(\varepsilon_{F}\right)}{\psi_{\uparrow}^{2}\left(\varepsilon_{F}\right)+\psi_{\downarrow}^{2}\left(\varepsilon_{F}\right)}
$$

where $\psi_{\sigma}(\varepsilon)$ are solutions of the Schrödinger equation with energy $\varepsilon$. For diffusive transport it is more appropriate to define a transport polarization

$$
s=\frac{\sigma_{\uparrow}-\sigma_{\downarrow}}{\sigma_{\uparrow}+\sigma_{\downarrow}} \equiv \frac{\rho_{\downarrow}-\rho_{\uparrow}}{\rho_{\downarrow}+\sigma_{\uparrow}}
$$

in terms of the spin dependent conductivities $\sigma_{\sigma}$ or resistivities $\rho_{\sigma}$.

\subsubsection{Mott analysis for $\mathrm{M} \perp j_{c}^{z}$}

A magnetization $\mathbf{M}$ in an arbitrary direction in the $x y$ plane may be written as

$$
\mathbf{M}=M \cos \phi \hat{\mathbf{x}}+M \sin \phi \hat{\mathbf{y}}
$$

where $\phi$ is the angle between $\mathbf{M}$ and the $x$ axis. From (3.11) we can write the spin polarization of the unperturbed medium as

$$
\mathbf{s}=s \cos \phi \hat{\mathbf{x}}+s \sin \phi \hat{\mathbf{y}}
$$

We are interested in the transverse response in the $x$ direction, it follows from (3.1) that

$$
\begin{aligned}
& \mathbf{s}^{\prime}=\beta s \cos \phi \hat{\mathbf{x}}+(\alpha+s \sin \phi) \hat{\mathbf{y}}-\gamma s \cos \phi \hat{\mathbf{z}} \\
& \mathbf{M} \times \mathbf{s}^{\prime}=-\frac{1}{2} \gamma \quad s M \sin 2 \phi \hat{\mathbf{x}}+\frac{1}{2} \gamma s M(1+\cos 2 \phi) \hat{\mathbf{y}} \\
& +\left(\alpha M \cos \phi+\quad \frac{1}{2} s M \sin 2 \phi-\frac{1}{2} \beta s M \sin 2 \phi\right) \hat{\mathbf{z}}
\end{aligned}
$$


Substituting this expression into (3.4) yields

$$
\begin{aligned}
& j_{s x}^{x}=-\frac{M \gamma^{\prime}}{2 \tau_{\mathrm{pr}}} \sin 2 \phi-\frac{\beta^{\prime}}{\tau_{\mathrm{sf}}} \cos \phi \\
& j_{s y}^{x}=\left(\frac{M \gamma^{\prime}}{2 \tau_{\mathrm{pr}}}-\frac{\alpha}{\tau_{\mathrm{sf}}}\right)+\frac{M \gamma^{\prime}}{2 \tau_{\mathrm{pr}}} \cos 2 \phi-\frac{s}{\tau_{\mathrm{sf}}} \sin \phi \\
& j_{s z}^{x}=\frac{\alpha M \cos \phi+\frac{1}{2} s M \sin 2 \phi-\frac{1}{2} \beta^{\prime} M \sin 2 \phi}{\tau_{\mathrm{pr}}}+\frac{M \gamma^{\prime}}{2 \tau_{\mathrm{sf}}} \cos \phi
\end{aligned}
$$

where $\left\{\beta^{\prime}, \gamma^{\prime}\right\}=\{\beta s, \gamma s\}$.

\subsubsection{Comparison of CPA potentials}

Each CPA calculation for an alloy such as $\mathrm{Ni}_{x} \mathrm{Fe}_{1-x}$ results in AS potentials, $v_{x}^{\mathrm{Fe}}$ and $v_{x}^{\mathrm{Ni}}$, for Fe and $\mathrm{Ni}$ that depend on the stoichiometry $x$. Since atomic potentials by themselves are not very illuminating, we place each of the potentials on a perfect fcc lattice with lattice constant $a_{x}$ and calculate the corresponding electronic structure. These are shown for $x=0.7,0.8$ and 0.9 in Fig. 3.7 where the $x$ dependence of the AS potentials is seen to be quite insignificant.

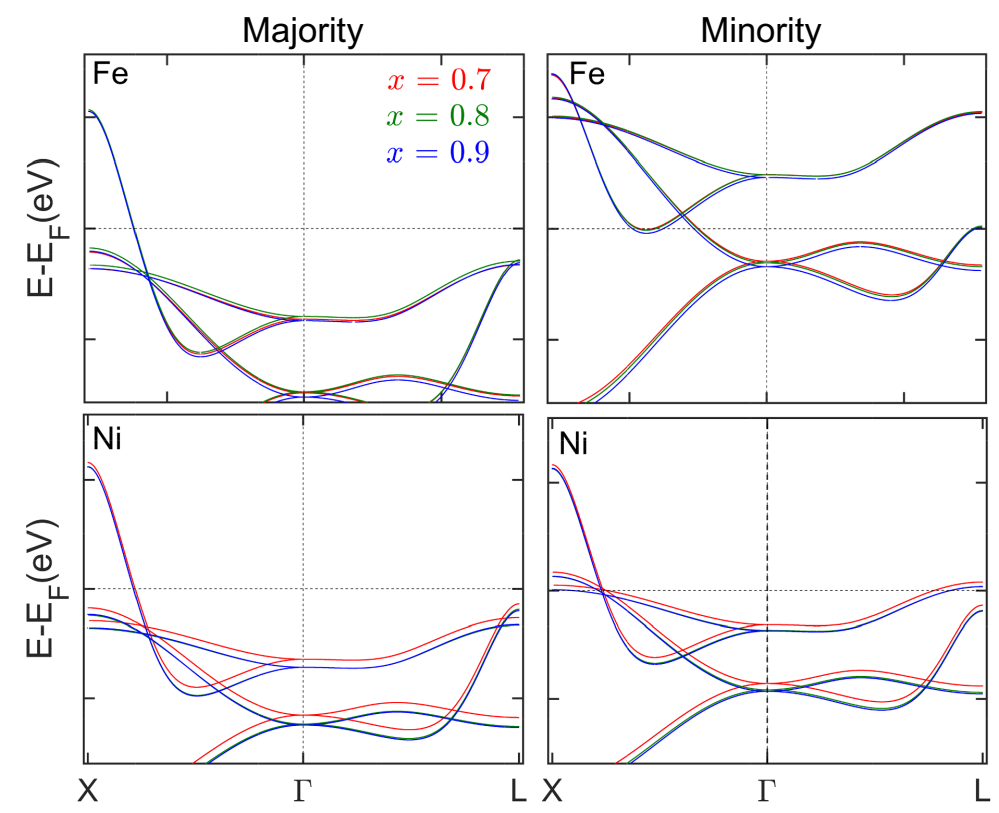

Figure 3.7: (lhs) Majority and (rhs) minority spin bands calculated for $\mathrm{Ni}$ and Fe AS potentials self-consistently using coherent potential approximation(CPA) for three different alloy concentrations, $x$ in $\mathrm{Ni}_{x} \mathrm{Fe}_{1-x}$. 


\section{4 \\ Fully resolved currents from quantum transport calculations}

We extract local current distributions from interatomic currents calculated using a fully relativistic quantum mechanical scattering formalism by interpolation onto a three-dimensional grid. The method is illustrated with calculations for Pt|Ir and Pt $\mid A u$ multilayers as well as for thin films of Pt and Au that include temperature-dependent lattice disorder. The current flow is studied in the "classical" and "Knudsen" limits determined by the sample thickness relative to the mean free path $\lambda$, introducing current streamlines to visualize the results. For periodic multilayers, our results in the classical limit reveal that transport inside a metal can be described using a single value of resistivity $\rho$ combined with a linear variation of $\rho$ at the interface while the Knudsen limit indicates a strong spatial dependence of $\rho$ inside a metal and an anomalous dip of the current density at the interface which is accentuated in a region where transient shunting is incomplete.

\subsection{Introduction}

The standard way to measure a bulk resistivity $\rho$ is the four-point-probe technique [158-160] which assumes isotropic current propagation. In the ongoing pursuit of miniaturization in electronics, such measurements have been extended to study the enhancement of resistivity in thin films [50, 161-167] (and wires [166, 168, 169])

"This chapter has been published as: R. S. Nair and P. J. Kelly, "Fully resolved currents from quantum transport calculations", Phys. Rev. B 103, 195406 (2021). 
with a common theme being the estimation of a single effective value of $\rho$ for a given thickness (radius) $d$ [169]. When the Fermi wavelength of the conduction electrons is comparable to $d$, the wave nature of electrons gives rise to finite size effects [58] that must be taken into consideration. If the mean-free-path $\lambda$ is comparable to $d$, the whole concept of a local resistivity becomes moot and as illustrated in Fig. 4.1(a), specular and diffusive reflection from surfaces play a role in determining the current distribution in such films $[50,51]$. The corresponding case of a multilayer is illustrated in Fig. 4.1(b) where interfaces give rise to specular and diffusive scattering and the finite transmission through interfaces leads to shunting of current which is typically addressed in experiments using parallel resistivity models $[52,170]$.

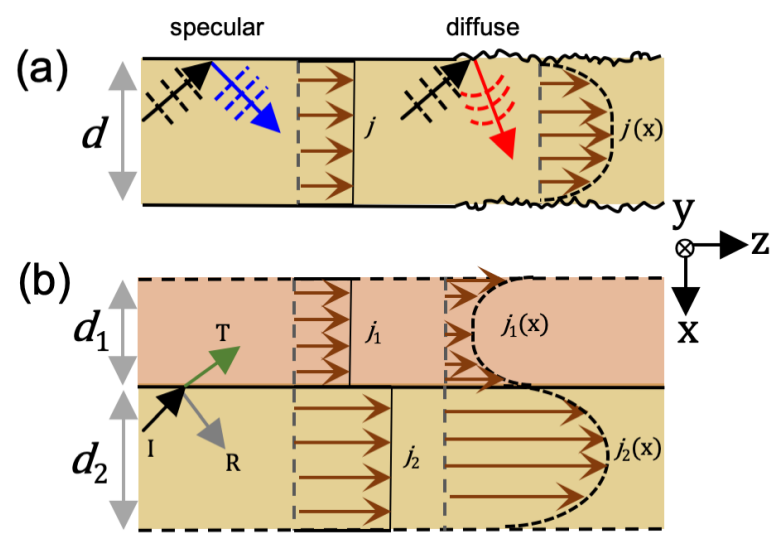

Figure 4.1: Schematics of (a) electron scattering in a thin film geometry of finite thickness $d$ illustrating the role of surface scattering. An electron wave-packet (depicted by the black arrows) undergoing only specular scattering at the surface (blue arrow) results in a uniform current distribution $j$ flowing in the $z$ direction whereas diffuse scattering from a rough surface (red arrow) results in a non-uniform current profile $j(x)$. (b) Scattering at an interface (thick black horizontal line) between two slabs of finite thicknesses $d_{1}$ and $d_{2}$ where the horizontal dashed lines represent a surface or the next interface in a bilayer/multilayer geometry. At an interface, a part of the incident electron wave (I) is transmitted (T) and the rest is reflected (R) specularly or diffusely eventually leading to current equilibration along $y$. A parallel resistivity model describes $x$-independent resistivities and, corresponding to these, uniform current distributions. A realistic current distribution $j_{1}(x)$ and $j_{2}(x)$ resulting from a resistivity gradient across the interface and from details of the scattering (specular and diffuse) is sketched on the right. Note that the spatial dependence is only expected to be significant over a length scale determined by the mean free path of the materials. Coordinate axes are shown for reference. 
The field of spintronics originated with multilayers comprising alternating thin films of magnetic and nonmagnetic metals $[5,6]$ and these continue to play a pivotal role $[43,76,77,171]$. The accurate estimation of various spin transport parameters is intimately connected with knowing how much charge current flows in the different layers that are of the order of 1-10 nm thick. A very recent attempt to determine this current distribution combined different thin film resistivity models with four-point-probe measurements for a large number of samples where the individual layer thicknesses were varied systematically [53]. This indirect approach was made necessary by the absence of a direct method to observe how current flows in the different layers of multilayer samples. Stejskal et al. concluded their study by emphasizing the need for more detailed structural characterization in order to be able to reduce the non-negligible variations they found in the model-dependent allocation of currents to individual layers.

Although the transport properties of metals are known to be dominated by states close to the Fermi energy [172-174], there have been few attempts to include the full complexities of the Fermi surfaces associated with partially filled $d$ bands in theoretical studies of transport parallel to the surfaces of thin films ${ }^{\dagger}$, or in the context of multilayers, parallel to the interfaces, the so-called current-in-plane (CIP) configuration [186-188]. The most sophisticated model used by Stejskal et al. [53] was the phenomenological electron gas model of Fuchs [50] and Sondheimer [51], generalized to treat two different surfaces [189] and to include transmission through interfaces between two different metals [170] as well as the effect of grain boundaries $[162,190]$. The purpose of this chapter is therefore to explore the possibility of calculating the spatial distribution of currents in realistic multilayers and thin films entirely from first principles including temperature-induced lattice disorder $[61,150]$. To do so, we introduce a discrete scheme to interpolate local currents [20] calculated using a fully relativistic DFT based scattering code [57] and apply it to evaluate the full spatial profile of currents in thin films of Pt and $\mathrm{Au}$ which are of interest to the spintronics community as well as in $\mathrm{Pt} \mid \mathrm{Au}$ and Pt|Ir multilayers.

The chapter is arranged as follows. In Sec. 4.2.1 some aspects of the scattering problem that are relevant for the calculation of interatomic currents are briefly summarized. The planar averaging introduced in Ref. 20 is relaxed in Sec. 4.2.2 to obtain fully spatially resolved local currents on a three dimensional grid. Inspired by fluid physics we introduce streamlines to visualize the current flow in Sec. 4.2.3. Although the same methodology can be straightforwardly applied to study the spatial distribution of spin currents, the present work will for simplicity

\footnotetext{
${ }^{\dagger}$ Most studies of electronic transport through thin films (or nanowires) based on realistic electronic structures have been in the ballistic regime [14, 15, 58]. Most have been for $\mathrm{Cu}$ [175-182] but calculations for Ag [178, 179], and Pt, Rh, Ir and Pd [183] thin films have also been reported. A simple but effective "brute force" approach to modelling the diffusive regime $[61,150]$ has to the best of our knowledge only been applied to the study of $\mathrm{Cu}$ thin films [184, 185]
} 
focus on charge currents. In Sec. 4.3 the methodology presented in the previous section is illustrated: in the Knudsen limit for a thin film of $\mathrm{Au}$ and a Pt|Au multilayer in Sec. 4.3.1; in the classical diffusive limit for a thin Pt film and a Pt|Ir multilayer in Sec. 4.3.2. The non-negligible effect of the choice of lead material is examined in Sec. 4.3.3 and we conclude with a brief discussion and outlook in Sec. 4.4.

\subsection{Methods}

\subsubsection{Quantum transport}

A typical two-terminal transport configuration is sketched in Fig. 4.2 with a scattering region $(\mathcal{S})$ sandwiched between ideal left $(\mathcal{L})$ and right $(\mathcal{R})$ crystalline leads. In the adiabatic approximation, atoms in the scattering region are displaced from their mean positions with a Gaussian distribution of displacements characterized by a root-mean square displacement $\Delta(T)$ chosen to reproduce the experimental resistivity [62] at a given temperature $T[61,150]$. Such disorder would break the translational symmetry completely and make it impossible to solve the Schrödinger equation. To remedy this, we introduce periodic boundary conditions in the $x y$ plane with an $N \times M$ "lateral supercell" comprising $N$ and $M$ unit cells in the $x$ and $y$ directions, respectively, whereby the disorder is assumed to be periodic. It turns out that remarkably small supercells are sufficient to eliminate observable effects of the residual periodicity as long as the temperature is not too low [57].

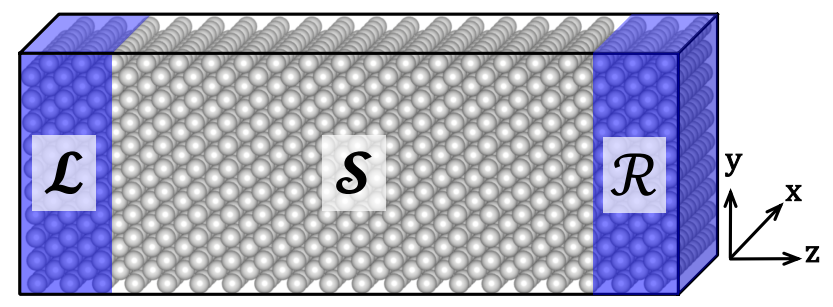

Figure 4.2: A scattering region $(\mathcal{S})$ is sandwiched between lattice-matched ballistic left $(\mathcal{L})$ and right $(\mathcal{R})$ leads which are semi-infinite in the $\pm z$-direction respectively. Superlattice periodicity is imposed in the $x y$ plane by means of an $N \times M$ supercell in the $x$ and $y$ directions, respectively. This construction makes it possible to simulate a wide range of disordered systems. Coordinate axes are shown for reference for an fcc lattice with $N=M=10$ and $x=[110], y=[1 \overline{1} 0], z=[001]$.

The transport problem now reduces to one of solving the single particle Schrödinger equation inside region $\mathcal{S}$ using the propagating Bloch states of the periodic semiinfinite leads as boundary conditions. To do so in practice, we make use of a "wave 
function matching" (WFM) scheme [56, 94, 102] formulated [16, 57] for a basis of tight binding (TB) muffin tin orbitals (TB-MTO) [67, 95, 147] and the atomic spheres approximation (ASA) [64]. TB-MTOs form a localized orbital basis $|i\rangle$ with $i=R l m s$ where $R$ is an atom site index and lms have their conventional meaning. In terms of the basis $|i\rangle$, the wavefunction $\Psi$ can be expressed as

$$
|\Psi\rangle=\sum_{i}|i\rangle\langle i \mid \Psi\rangle
$$

and the Schrödinger equation becomes a matrix equation with matrix elements $\langle i|H| j\rangle . \Psi$ is a vector of coefficients with elements $\psi_{i} \equiv\langle i \mid \Psi\rangle$ extending over all sites $R$ and over the orbitals on those sites, for convenience collectively labelled as $i_{R} .\left|\Psi_{R}\right\rangle$ is a projection of the total wave function $|\Psi\rangle$ onto the orbitals on atom $R$

$$
\left|\Psi_{R}\right\rangle=\sum_{i_{R}}\left|i_{R}\right\rangle\left\langle i_{R} \mid \Psi\right\rangle
$$

The minimal TB-MTO basis along with the local density approximation (LDA) of density functional theory (DFT) $[54,55]$ makes the scattering problem tractable for scattering regions comtaining $10^{4}-10^{5}$ atoms. A detailed description of the TBMTO-WFM transport scheme can be found in references [16] and [57].

\subsubsection{Interpolation of interatomic currents onto a three dimensional grid}

We begin with expressions [20] for the charge current $j_{c}^{P Q}$ and spin current $j_{s \alpha}^{P Q}$ between atoms $P$ and $Q$

$$
\begin{aligned}
& j_{c}^{P Q}=\frac{1}{i \hbar}\left[\left\langle\Psi_{P}\left|H_{P Q}\right| \Psi_{Q}\right\rangle-\left\langle\Psi_{Q}\left|H_{Q P}\right| \Psi_{P}\right\rangle\right] \\
& j_{s \alpha}^{P Q}=\frac{1}{i \hbar}\left[\left\langle\Psi_{P}\left|\sigma_{\alpha} H_{P Q}\right| \Psi_{Q}\right\rangle-\left\langle\Psi_{Q}\left|H_{Q P} \sigma_{\alpha}\right| \Psi_{P}\right\rangle\right]
\end{aligned}
$$

that are given in terms of the block $H_{P Q}$ of Hamiltonian matrix elements and vectors of expansion coefficients $\left\langle i_{P} \mid \Psi\right\rangle$ and $\left\langle i_{Q} \mid \Psi\right\rangle$ obtained by solving the scattering problem. A summation over $l m s$ is implicit. $\boldsymbol{\sigma}$ is a vector of Pauli spin matrices $\sigma_{\alpha}$ and $\alpha$ labels the polarization direction of the spin current. The materials whose transport properties we wish to study are crystalline materials or substitutional alloys at finite temperatures whose constituent atoms are displaced at random from the sites of a Bravais lattice. Determining the spatial distribution of currents in the scattering region thus requires interpolating all $j^{P Q}$ onto regular real space meshes as a function of $x, y, z$.

In Fig. 4.3 we illustrate the discretization of an arbitrary transport geometry. To generalize the interpolation and averaging of currents for an arbitrary geometry generated by translation vectors $\mathbf{T}_{1}, \mathbf{T}_{2}$ and $\mathbf{T}_{3}$ that are not necessarily orthogonal 


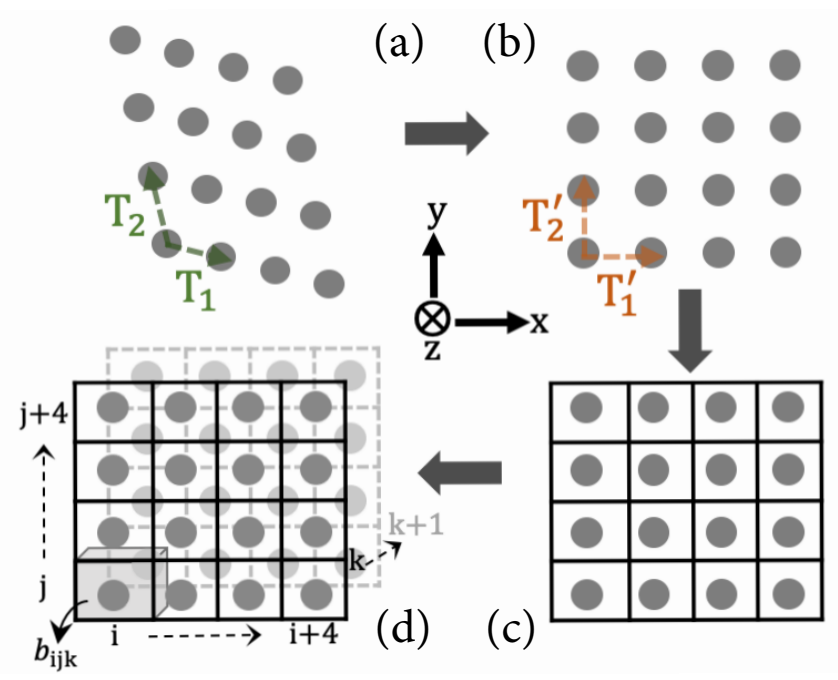

Figure 4.3: (a) An arbitrary lattice described by the translation vectors $\mathbf{T}_{1}, \mathbf{T}_{2}$ (and $\mathbf{T}_{3}$; not shown) is affine transformed into an equivalent orthogonal lattice (b) described by $\mathbf{T}_{1}^{\prime}, \mathbf{T}_{2}^{\prime}$ (and $\mathbf{T}_{3}^{\prime}$; not shown). (c) The lattice is then discretized into $n_{x} n_{y} n_{z}$ boxes equal to the number of atoms. Here $n_{i}$ with $i=x, y, z$ is the number of atomic layers in the $i$ th direction. (d) The final grid where a single box $b_{i j k} \equiv b\left(x_{i}, y_{j}, z_{k}\right)$ is explicitly shown in 3D to help visualize the grid. The example shown is for a $4 \times 4$ lateral supercell in the $x y$ plane with only two consecutive layers in the $z$ direction shown for clarity. Cartesian coordinate axes are shown for reference.

to each other, we first perform an affine transformation $\mathcal{T}$ [191-194] of the translation vectors with a combination of shearing and scaling transformations into a dual space of orthonormal vectors $\mathbf{T}_{1}^{\prime}, \mathbf{T}_{2}^{\prime}$ and $\mathbf{T}_{3}^{\prime}$ that lie along the Cartesian $x, y$ and $z$ coordinate axes. Mathematically, we can express this as

$$
\mathcal{T}: \mathcal{R} \rightarrow O
$$

where $\mathcal{R}$ is the parent coordinate space and $O$ is the dual space. Since the collinearity of points along a given direction in the parent space is mapped into the corresponding direction in the affine transformed space ( $\$ 23$ of [191]), averaging of local quantities in $O$ say $x\left(\equiv \mathbf{T}_{1}^{\prime}\right)$ can be treated as being equivalent to averaging in the direction of the corresponding translation vector $\left(\mathbf{T}_{1}\right)$ in $\mathcal{R}$. We now apply $\mathcal{T}$ to the disordered geometry and map all atomic coordinates from $\mathcal{R}$ to $O$ thus transforming the disorder from the parent space. The disordered geometry in $O$ is then divided into boxes whose dimensions $D_{x}, D_{y}$ and $D_{z}$ are determined from the average distance between consecutive atomic layers in the $x, y$ and $z$ directions, respectively, such that the number of boxes is equal to the number of atoms and each box contains exactly one atom; the latter is guaranteed if the temperature-induced 
atomic displacements are much less than the interatomic separations. The regular lattice of boxes is constructed in such a way that the centres of gravity of the atomic coordinates and boxes coincide.

$\mathbf{j}^{P Q} \equiv\left(j_{c}^{P Q}, j_{s x}^{P Q}, j_{s y}^{P Q}, j_{s z}^{P Q}\right)$ is imagined as a current through a wire connecting the positions of atoms $P$ and $Q$ with (arbitrary) cross section $A_{P Q}$, see Fig. 4.4. Since microscopic details of the spatial distribution of $\mathbf{j}^{P Q}$ are unknowable, we assume a homogeneous flux of current between $P$ and $Q$. The tensor current density of the wire is such that $\overleftrightarrow{j}^{P Q} V_{P Q}=\mathbf{j}^{P Q} \otimes \mathbf{d}_{P Q}$ where $V_{P Q}=A_{P Q} d_{P Q}$ is the volume of the wire $P Q, \mathbf{d}_{P Q}$ is the vector pointing from $P$ to $Q$ and $d_{P Q}$ its length. The direct product $\mathbf{j}^{P Q} \otimes \mathbf{d}_{P Q}$ is estimated in the parent space $\mathcal{R}$ as it depends on the components of $\mathbf{j}^{P Q}$ and $\mathbf{d}_{P Q}$. Since the affine transformation $\mathcal{T}$ preserves ratios of distances between points in a line ( $\$ 24$ of [191]), the current contribution to each box can be determined in the dual space $O$ using a linear interpolation scheme.

Unlike the planar averaged scheme [20] where a one-dimensional interpolation was enough to evaluate the variation in the $z$ direction only, a three dimensional interpolation is implemented here. A general case is shown in Fig. 4.4 for a pair of atoms $P$ and $Q$ in $O$ with their centres at $\left(x_{P}, y_{P}, z_{P}\right)$ and $\left(x_{Q}, y_{Q}, z_{Q}\right)$ respectively. Note that $P$ and $Q$ can be inside or outside the box " $b$ ". Only boxes that are intersected by the wire $P Q$ receive a contribution from the current $\mathbf{j}^{P Q}$ which makes it a classic computational problem of "collision detection" where one seeks the point of intersection of the path of an object and a surface of interest. Mathematically, this requires simultaneously solving the equation of line $P Q$ connecting the pair of atoms $\{P, Q\}$ with equations describing the six faces of each box for all possible $\{P, Q\}$. This amounts to $7 \times C(n, 2) \times C(n, 1)=\frac{7}{2}\left(n^{3}-n^{2}\right)$ equations where $n$ is the number of atoms in the geometry with a computational effort that scales as $\sim O\left(n^{9}\right)$. To efficiently perform interpolations for systems with multiple configurations of $10^{4}-10^{5}$ atoms, we instead take advantage of the orthogonality of the dual space $O$ and employ a line clipping algorithm [195] to determine all boxes which are intersected by wire $P Q$ as well as the points of intersection $U$ and $V$.

The line $P Q$ is first parametrized as

$$
(x, y, z)=\left(x_{P}, y_{P}, z_{P}\right)-c\left(x_{P}-x_{Q}, y_{P}-y_{Q}, z_{P}-z_{Q}\right)
$$

for $0 \leq c \leq 1$. Each box can be described by six boundaries, two for each direction: $\left\{x_{-}, x_{+}\right\},\left\{y_{-}, y_{+}\right\},\left\{z_{-}, z_{+}\right\}$, allowing us to write six inequalities for $P \neq Q$

$$
\frac{\alpha_{P}-\alpha_{+}}{\alpha_{P}-\alpha_{Q}} \leq c \leq \frac{\alpha_{P}-\alpha_{-}}{\alpha_{P}-\alpha_{Q}} \quad \text { for } \quad \alpha=x, y, z
$$

Equation (4.7) is only satisfied by the points on the line $P Q$ that lies inside the box. Then $c$ takes a range of continuous values from which the intercepts $U$ and $V$ can 


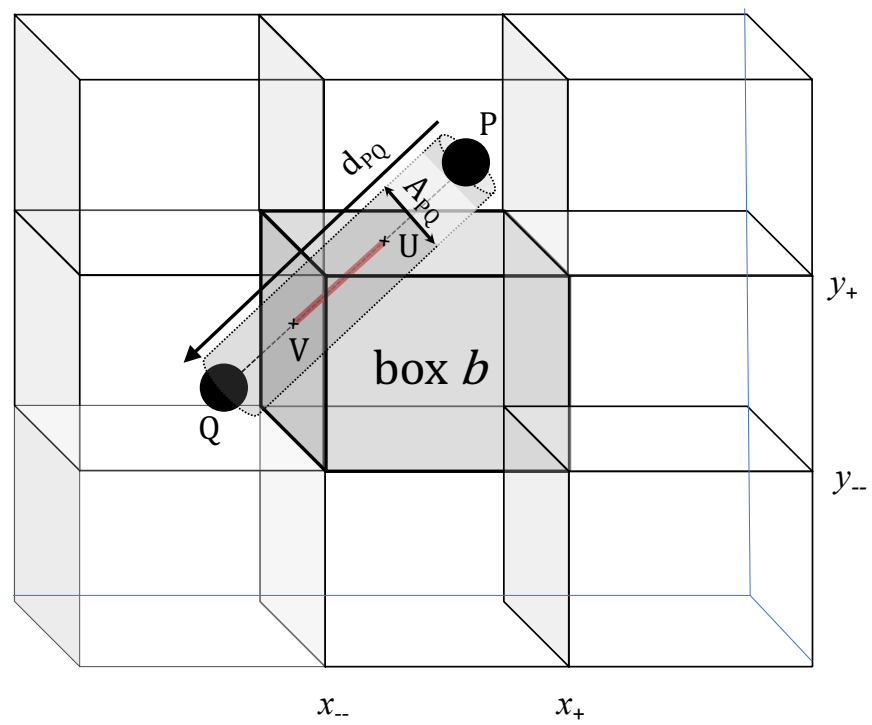

Figure 4.4: Illustration of the discrete current scheme where the grid is zoomed to show how the current $j^{P Q}$ between atoms $P$ and $Q$ is interpolated into the box $b$. The current contribution from "wire" $P Q$ to the box $b$ comes from the segment $U V$ (coloured red) that lies inside the box.

be obtained as

$$
\begin{aligned}
& U=\left(x_{P}, y_{P}, z_{P}\right)-\min (c)\left(x_{P}-x_{Q}, y_{P}-y_{Q}, z_{P}-z_{Q}\right) \\
& V=\left(x_{P}, y_{P}, z_{P}\right)-\max (c)\left(x_{P}-x_{Q}, y_{P}-y_{Q}, z_{P}-z_{Q}\right)
\end{aligned}
$$

Note that $U=\left(x_{P}, y_{P}, z_{P}\right)$ when $P$ lies inside $b$ and $V=\left(x_{Q}, y_{Q}, z_{Q}\right)$ when $Q$ lies inside $b$, which we denote as $P \in b, Q \in b$, respectively in the following. We define a parameter $\beta$ that indicates how much of the wire lies outside the box on either side.

$$
\begin{aligned}
& \beta_{Q P, b}= \begin{cases}0 & \text { if } Q \in b \\
\frac{d_{Q V}}{d_{Q P}} & \text { if } Q \notin b\end{cases} \\
& \beta_{P Q, b}= \begin{cases}0 & \text { if } P \in b \\
\frac{d_{P U}}{d_{P Q}} & \text { if } P \notin b\end{cases}
\end{aligned}
$$

where $d_{A B}$ is the norm of the vector $\mathbf{d}_{A B}$ pointing from point $B$ to point $A$. (In the planar averaged scheme [20], only the projection $d_{A B}^{z}$ of $\mathbf{d}_{A B}$ on $z$, is used to evaluate $\beta_{P Q}$.) Since the current changes between $Q$ and $P$, we make a linear interpolation

$$
\mathbf{j}^{P Q}(c)=c \mathbf{j}^{P Q}-(1-c) \mathbf{j}^{Q P}
$$


Thus, for a box of volume $V_{b}$, the contribution from $\overleftrightarrow{j}^{P} Q_{V_{P Q}}$ is given by

$$
\begin{aligned}
& \int_{\beta_{Q P, b}}^{1-\beta_{P Q, b}} \quad \mathbf{j}^{P Q}(c) \otimes \mathbf{d}_{P Q} d c= \\
& \frac{1}{2} \quad\left[\left(1-\beta_{P Q, b}\right)^{2}-\left(\beta_{Q P, b}\right)^{2}\right] \mathbf{j}^{P Q} \otimes \mathbf{d}_{P Q} \\
& +\frac{1}{2} \quad\left[\left(1-\beta_{Q P, b}\right)^{2}-\left(\beta_{P Q, b}\right)^{2}\right] \mathbf{j}^{Q P} \otimes \mathbf{d}_{Q P} .
\end{aligned}
$$

Note that $\mathbf{d}_{P Q}=-\mathbf{d}_{Q P}$. The average current density tensor in the box $b$ is then

$$
\stackrel{\leftrightarrow}{j_{b}}=\frac{1}{V_{b}} \sum_{P, Q} \frac{1}{2}\left[\left(1-\beta_{P Q, b}\right)^{2}-\left(\beta_{Q P, b}\right)^{2}\right] \mathbf{j}^{P Q} \otimes \mathbf{d}_{P Q}
$$

and we take this value of $\overleftrightarrow{j_{b}}$ to be the average current density at the centroid of $b$. By interpolating all interatomic currents into all boxes $b$, we obtain the complete spatial variation of the current density. Multiplying the current density with cross sectional area of the box $b$ perpendicular to $z$, we get current per unit voltage applied between leads, a conductance. Summation of the normalized currents of all boxes lying in a given $x y$ plane should then be equal to the Landauer-Buttiker conductance that is calculated independently of the interatomic currents and interpolation scheme. This provides a check of the whole local current formalism. Finally, centroids of the boxes are affine transformed back into the parent space,

$$
\mathcal{T}^{\prime}: O \rightarrow \mathcal{R}
$$

We observe spatial oscillations in calculated spin currents because of the interference between reflected and incident electron matter waves. Although these oscillations are real, they are not present in semiclassical descriptions of transport. Because they are found to be attenuated away from the leads in parallel with the corresponding decrease in the unscreened particle accumulation, we follow Ref. 20 and use the latter to reduce these quantum fluctuations to facilitate analysis using semiclassical transport formulations. Since lateral supercell sizes do not exceed more than a few hundred atoms in our calculations, we perform such averaging using only the planar averaged unscreened particle accumulation. Details of this averaging can be found in Ref. 20.

\subsubsection{Current streamlines}

From here on we only consider the charge current and will therefore drop the subscript $c$. To study transport in the lateral direction, we will construct periodic bilayer in the $x$ direction (see Fig. 4.5). Because of the assumed superlattice periodicity, the average current in the $y$ direction, $\bar{j}_{y}(x, z)=0$. The visualization of $\mathbf{j}(\mathbf{r})$ reduces to a problem in two dimensions if we average over $y$ and, to do so, we 
introduce a current stream function $\psi(x, z)$ (not to be confused with the wavefunction) by analogy with the velocity stream function in fluid physics [196]. In the steady state, charge conservation requires that $\nabla . \mathbf{j}=0$ and for the $x z$ plane, this reduces to

$$
\frac{\partial j_{x}}{\partial x}+\frac{\partial j_{z}}{\partial z}=0
$$

Defining $\psi(x, z)$ such that

$$
j_{x}=\frac{\partial \psi}{\partial z} \quad \text { and } \quad j_{z}=-\frac{\partial \psi}{\partial x}
$$

automatically leads to (4.14) being satisfied. $\psi(x, z)=$ constant is a path whose tangent at any point gives the direction of the current vector $\mathbf{j}=j_{x} \mathbf{i}+j_{z} \mathbf{k}$ at that point. This defines a streamline and the volume flow per unit width between streamlines connecting the left $(\mathcal{L})$ and right $(\mathcal{R})$ leads is

$$
\psi_{\mathcal{R}}-\psi_{\mathcal{L}}=\int_{\mathcal{L}}^{\mathcal{R}} d \psi=\int_{\mathcal{L}}^{\mathcal{R}}\left[j_{x} d z-j_{z} d x\right]
$$

This region can be thought of as a conducting strip carrying a constant flux of current analogous to a streamtube for incompressible fluid flow such that crowding of streamlines at a region in the flow-field indicates a local increase in the magnitude of the current [196].

\subsubsection{Mean Free Path}

In the relaxation time approximation (RTA), the conductivity is given in terms of the $\mathbf{k}$ dependent velocities $\boldsymbol{v}(\mathbf{k})=\frac{1}{\hbar} \nabla_{\mathbf{k}} \varepsilon(\mathbf{k})$ as

$$
\sigma_{i j}=e^{2} \iiint \frac{d^{3} k}{8 \pi^{3}} \tau(\mathbf{k}) v_{i}(\mathbf{k}) v_{j}(\mathbf{k})\left(-\frac{\partial f}{\partial \varepsilon}\right)_{\varepsilon=\varepsilon(\mathbf{k})} .
$$

In the low temperature limit $-\frac{\partial f}{\partial \varepsilon} \rightarrow \delta\left(\varepsilon-\varepsilon_{F}\right)$ and (4.17) becomes an integral over the Fermi surface $S_{F}$. Assuming additionally that $\tau(\mathbf{k})=\tau(\varepsilon(\mathbf{k}))$ then

$$
\sigma=e^{2} D\left(\varepsilon_{F}\right) \tau\left(\varepsilon_{F}\right)\left\langle v_{F}^{2}\right\rangle
$$

where $D(\varepsilon)$ is the density of states. Both $D\left(\varepsilon_{F}\right)$ and $\left\langle v_{F}^{2}\right\rangle$ can be evaluated from standard bulk LMTO electronic structure calculations ${ }^{*}$ and since $\sigma \equiv 1 / \rho$ is known

\footnotetext{
"We use Mark van Schilfgaarde’s "lm" extension of the Stuttgart LMTO (linear muffin tin orbital) code that includes spin-orbit coupling and is maintained in the Questaal suite at https://www.questaal.org
} 
$[62]^{\S}, \tau$ can be expressed as

$$
\tau=\frac{\sigma}{e^{2} D\left(\varepsilon_{F}\right)\left\langle v_{F}^{2}\right\rangle}
$$

and the mean free path can be estimated as $\lambda=\tau\left\langle v_{F}^{2}\right\rangle^{1 / 2}$.

\subsection{Results}

Different regimes of electron transport can be identified depending on the ratio of the electron mean free path $\lambda$ to the critical dimension $d$ of the scattering geometry that is the Knudsen number $(\mathrm{Kn})$. When $\lambda \ll d$, we are in the classical limit where the flow of current is well described by Ohm's law. The other extreme is the Knudsen limit where $\lambda \gg d$, size effects and interface or surface scattering dominate and transport deviates from Ohm's law [197].

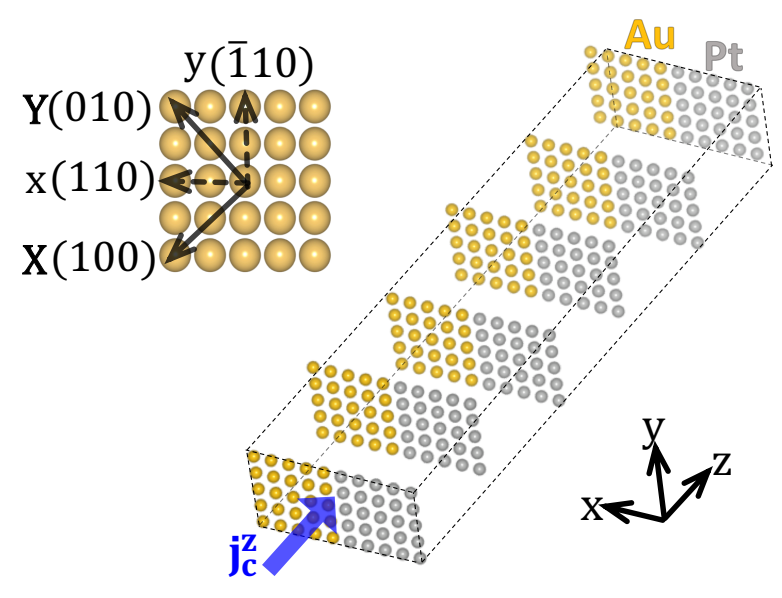

Figure 4.5: Sketch of an $N \times M$ lateral supercell used to model transport in a lattice-matched $\mathrm{AB}$ multilayer with $\mathrm{A}=\mathrm{Au}$ and $\mathrm{B}=\mathrm{Pt}$. For clarity, only six layers in the $z$ direction are explicitly shown, the separation of the layers is exaggerated and the leads sandwiching this geometry in the $\pm z$-directions are not shown. The $x$ direction is the crystal [110] direction. Part of an fcc layer perpendicular to the [001] direction with in-plane crystallographic directions is shown on the left. The conventional cubic axes of an fcc lattice are $X, Y$ and $Z(\equiv z)$.

\footnotetext{
${ }^{\S}$ We could calculate the conductivity entirely from first principles; see [61, 174] but because the results are not always in as good agreement with experiment as in [61], it is more expedient to choose the thermal disorder to reproduce the experimental resistivity (and magnetization) as in $[20,34,48,57,61]$
} 
To illustrate the three-dimensional current scheme presented above, we consider thin films and two-component ...A $|\mathrm{B}| \mathrm{A} \mid \mathrm{B} \ldots$ multilayers where the thickness of the $i$ th layer is $d_{i}$. In this chapter, only fcc metals are considered as sketched schematically in Fig. 4.5 with a charge current flowing in the [001] direction, parallel to the $\mathrm{A} \mid \mathrm{B}$ interfaces.

A k-point sampling of $\sim \frac{160}{N} \times \frac{160}{M}$ for an $N \times M$ supercell is used throughout the chapter. From now on we use the terms current and current density interchangeably. Unless stated otherwise, all currents are averaged over $y$ and are calculated for a temperature $T=300 \mathrm{~K}$ ("room temperature"); when averaging currents over the scattering region, $z \in \mathcal{S}$, a few layers close to the leads where transient effects are observed are omitted.

\subsubsection{Knudsen limit}

\section{Au thin film}

We begin by calculating the current in a free-standing [110] oriented thin film of Au. The thin film is modelled as a Aulvacuum "multilayer" by alternating 60 atomic layers of Au with five layers of "empty spheres" (with nuclear charge $Z=0$ to simulate vacuum [198-200]) in the $x$ direction so that $N=60+5$. A periodicity of $M=3$ layers in the $y$ direction is imposed and the scattering region is 90 atomic layers thick in the $z$ direction, see Fig. 4.5. A value of the root-mean square displacement $\Delta$ of the atoms in the scattering region was chosen to reproduce the room temperature bulk resistivity of $\mathrm{Au}, \rho_{\mathrm{Au}}^{300}=2.3 \pm 0.07 \mu \Omega \mathrm{cm}[62]^{\dagger}$. The thickness of the slab in the $x$ direction is approximately $\frac{1}{2} \lambda_{\mathrm{Au}}^{300}$ where $\lambda=34.5 \mathrm{~nm}$ was estimated in the RTA as described above. Ballistic Au leads were used to minimize transient effects at the lead|scattering region interface, between crystalline and thermally disordered Au.

In Fig. 4.6(a) we plot the charge current $\bar{j}_{z}(x)$ flowing in the $z$ direction averaged over the $y$ and $z$ directions as a function of $x$ (black symbols). This shows a gradual concentration of the current away from the surfaces and towards the middle of the film. A strong $z$ dependence is also apparent from plots of $\bar{j}_{z}\left(x, z_{0}\right)$ averaged over $y$ and $z=z_{0} \pm 5$ atomic layers for different values of $z_{0} \sim 3,5,7,9 \mathrm{~nm}$ measured from the left lead. The current streamlines are plotted in Fig. 4.6(b) where they are superimposed on a colour map showing the magnitude of the charge current. The colour map shows a larger current density at the centre of Au in both $x$ and $z$ directions. On closer examination, streamlines are not parallel to the $z$ axis but exhibit curvature. This demonstrates that the current distribution has not reached its asymptotic form in $z$ which is not surprising as the length of the scattering region

\footnotetext{
'The value of root-mean-square displacement $\Delta$ chosen with a view to reproducing the room temperature resistivity of pure Au turned out to yield a value of $\rho_{\mathrm{Au}}=2.6 \pm 0.07 \mu \Omega \mathrm{cm}$ corresponding to $T \sim 335 \mathrm{~K}$. Since this has no consequences for the present study, it was not corrected.
} 


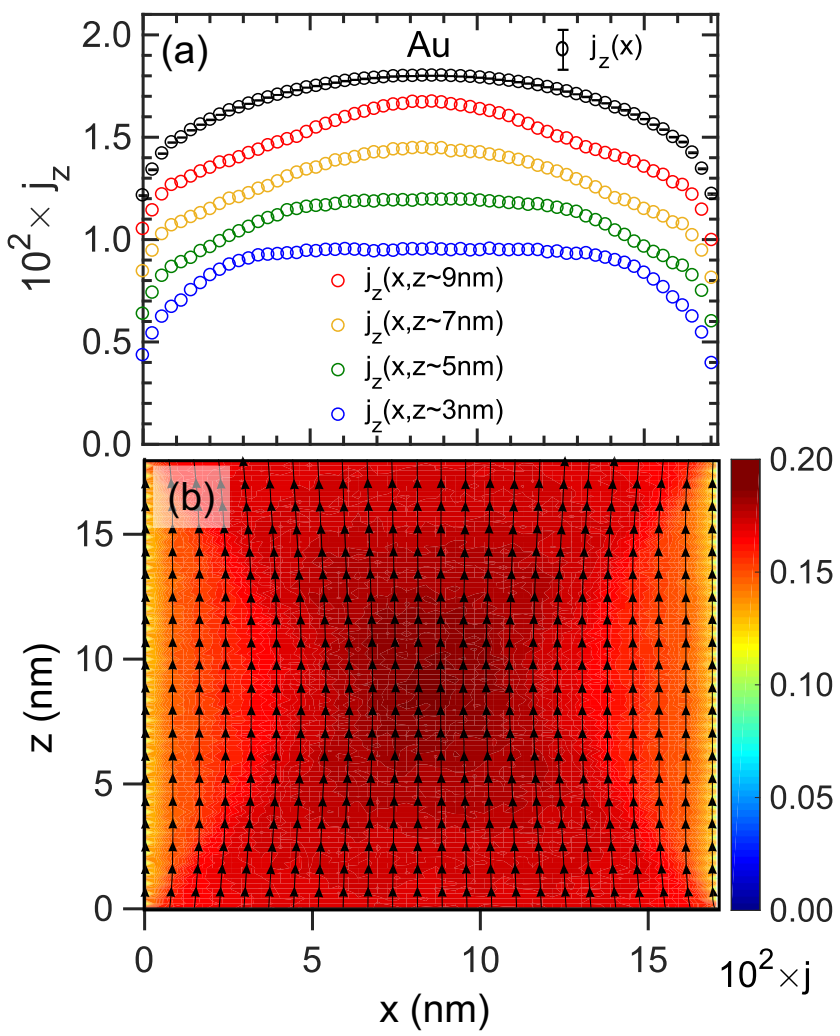

Figure 4.6: Current distribution in a thin film of Au at 335K. (a) Top curve, black symbols: current density $\bar{j}_{z}(x)$ obtained by averaging over $y$ and $z$. Colour symbols: $\bar{j}_{z}\left(x, z_{0}\right)$ obtained by averaging over $y$ and $z=z_{0} \pm 5$ layers in the $z$ direction for four different values of $z_{0}$ that are offset from the black curve in steps of $\Delta j=0.002$ for clarity. The error bars, that are smaller than the symbol size, indicate the average deviation over 10 random configurations of disorder. (b) Streamlines of the current vector $\mathbf{j}(x, z)=j_{x} \mathbf{i}+j_{z} \mathbf{k}$ in the $x z$ plane. The colour contour in the background corresponds to the magnitude of the current.

is only about half of the mean free path $\lambda^{*}$. Apart from a rapid decay of the current density within a few layers of the surface that is described by a "specularity coefficient" $p$ in the Fuchs-Sondheimer framework [50, 51], an approximately linear variation of the conductivity from the surface to the middle of the film is observed at the centre of the scattering region furthest from the leads. An "effective resistivity" clearly conceals substantial variation in the current density for film thicknesses

\footnotetext{
The relatively short scattering region studied here is a consequence of treating a very large lateral supercell containing $(60+5) \times 90 \times 3=17550$ atoms including spin-orbit coupling taking us to the current limits of our computing facilities in terms of both memory and run time
} 
that are commonly used in spintronics.

\section{Pt|Au multilayer}

Modern experiments frequently use heterostructures in which the thicknesses $d_{i}$ of constituent layers are comparable to the corresponding bulk mean free paths $\lambda_{i}$. To demonstrate the deviation from bulk behaviour we choose a Pt|Au multilayer. Both Pt and $\mathrm{Au}$ are fcc metals with only a $2 \%$ lattice mismatch so that such an epitaxial multilayer might be prepared without undue structural disorder. Thermal disorder corresponding to the room temperature bulk resistivities of $\mathrm{Au}$, $\rho_{\mathrm{Au}}^{300}=2.6 \pm 0.07 \mu \Omega \mathrm{cm}$ and Pt, $\rho_{\mathrm{Pt}}^{300}=10.8 \pm 0.5 \mu \Omega \mathrm{cm}$, was used with different mean square displacements in the Au and Pt layers. The mean free path in $\mathrm{Au}$, $\lambda_{\mathrm{Au}}^{300}=34.5 \mathrm{~nm}$ is almost ten times that in Pt, $\lambda_{\mathrm{Pt}}^{300}=3.74 \mathrm{~nm}$ [201]. Choosing $d_{\mathrm{Au}}<\lambda_{\mathrm{Au}}^{300}$ and $d_{\mathrm{Pt}}>\lambda_{\mathrm{Pt}}^{300}$ should make any size effect apparent at room temperature. We construct a scattering geometry as shown schematically in Fig. 4.5 with 60 atomic layers each of $\mathrm{Pt}$ and $\mathrm{Au}$ in the $x$ direction with a periodicity of 3 layers in the $y$ direction and 90 layers thick in the $z$ direction corresponding to a total of 32400 atoms in the scattering region. A charge current is injected from ballistic Au leads in the $z$ direction. The resulting charge current distribution in the Pt|Au multilayer is plotted in Fig. $4.7^{\S}$ where the error bars, that are smaller than the symbol sizes, correspond to the uncertainty with which the experimental resistivities are reproduced in our scattering calculations.

The average shunting $\bar{j}_{z}^{\mathrm{Au}} / \bar{j}_{z}^{\mathrm{Pt}} \sim 2.3$ i.e, the ratio of the mean current value in $\mathrm{Au}$ to that in Pt, indicated by the solid black horizontal lines in each layer plotted in Fig. 4.7(a), is much lower than expected from the ratio of the bulk resistivities $\rho_{\mathrm{Pt}}^{300} / \rho_{\mathrm{Au}}^{300} \sim 4$.2. The charge current is seen to be constant and saturated inside $\mathrm{Pt}$ while in $\mathrm{Au}$ a rapid increase in the two atomic layers adjacent to the interface followed by a continuous variation to the centre of the layer is observed. We note a small, anomalous dip in the current density in the Pt layers next to the interface. The current density variation at the interface and in the Au slab is clearly not amenable to description using a single resistivity. An almost immediate saturation of the total current carried by the Au and Pt layers is observed when these are plotted as a function of $z$ in Fig. 4.7(b) making our results independent of the length of the scattering region. The streamlines plotted in Fig. 4.7 are parallel to the $z$ axes inside Pt indicating that there is no net flow of current across the interface into $\mathrm{Au}$ as asymptotic shunting of the total charge current density is reached in the $z$ direction. However, a redistribution of the current inside $\mathrm{Au}$ is visible from the color

\footnotetext{
${ }^{\S}$ Our interatomic currents are normalized to the applied bias between the left and right leads. After interpolating these onto a regular mesh, the currents are normalized to the conductance in the $z$ direction. When summed over $x$ and $y$, they are therefore dimensionless and equal to unity at any position $z$ to satisfy charge current conservation. $x$ and $y$ averaging is simply given by adding all the currents in the $x y$ plane
} 


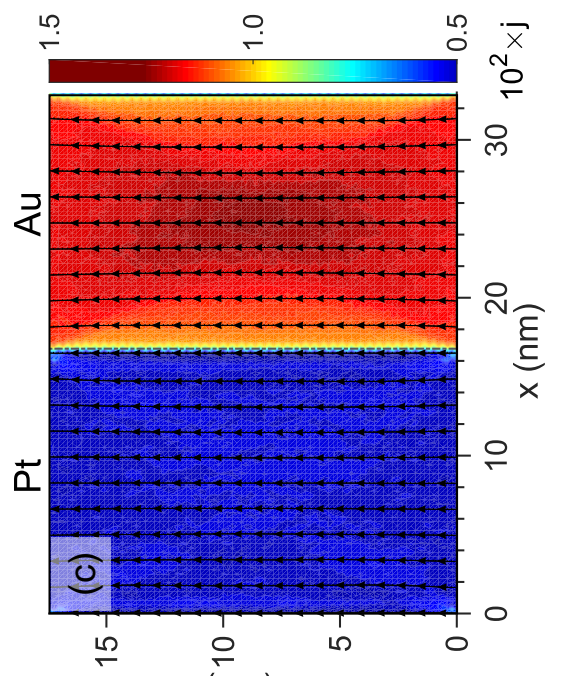

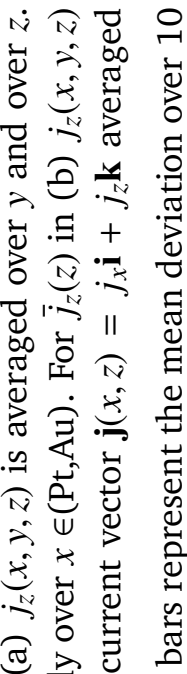

(mu) z

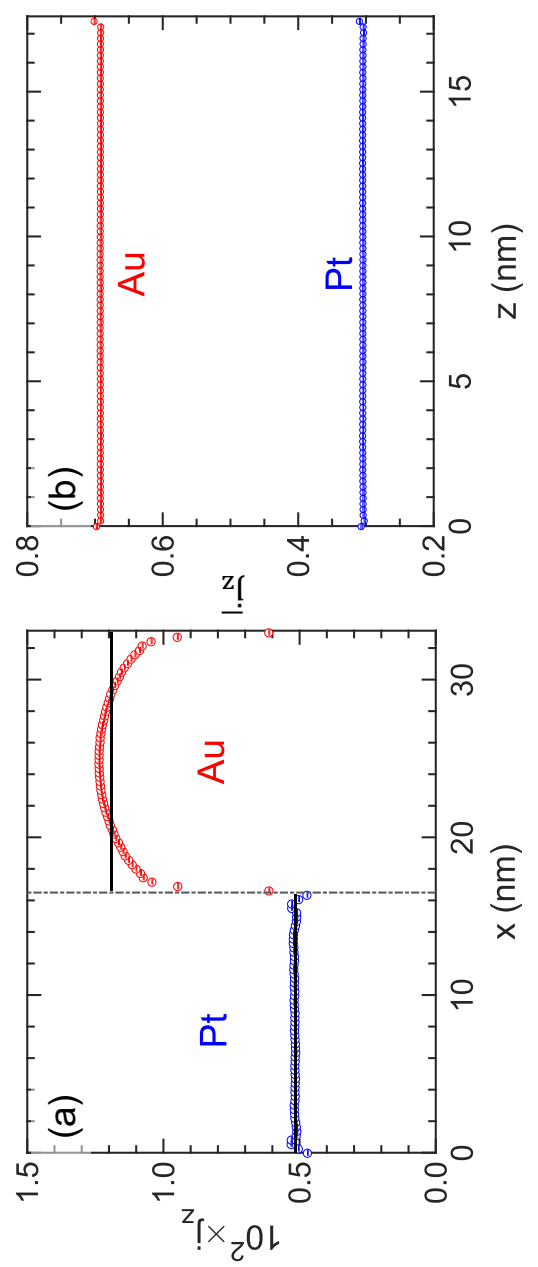

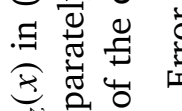

1. D 0

官

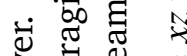

奈离昰

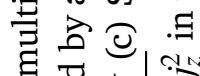

至离离营

๘ 0 \&

ヨ\& \& II

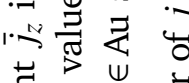

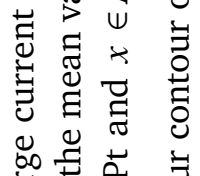

売芯

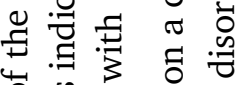

Чั้

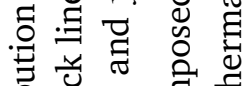

节芯

氙至究

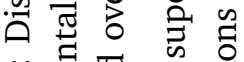

듀 Ð

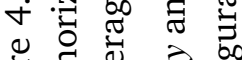

늘 닐

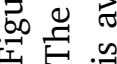

ठे 
contour and small curvature of the streamlines that is similar to what we saw for the Au thin film. As the total current in each layer is independent of $z$, one might use a parallel resistance model to estimate the current shunting noting, however, that this would not correctly describe microscopic details of the variation near the interface and in the Au layer just presented.

\subsubsection{Classical limit}

The classical diffusive limit is achieved when the mean free path is much shorter than other critical dimensions of the structures being studied, $\lambda \ll d$. Memory requirements currently limit the lateral supercell size to $H=N \times M \approx 300-400$ atoms for which the maximum length of scattering region is about $L \approx 130$ atoms when spin-orbit coupling is included. Because the computational effort for metallic systems scales as approximately $H^{2} L[16,57]$, considerably longer scattering regions can be studied with smaller lateral supercells. To attain the diffusive limit we need to consider high resistivity materials or study elevated temperatures or both.

\section{Pt thin film}

We first study charge transport through a thin film of Pt, modelling a free-standing [110] oriented Pt layer as a Pt|vacuum multilayer with $N$ layers of Pt and 5 layers of "empty spheres" repeated periodically in the $x$ direction. The effect of increasing the default periodicity of three atomic layers in the $y$ direction to five atomic layers is studied. To minimize lead|scattering- region interface effects, Pt leads are used. We calculate the average resistivity for different values of $N$ and identify the thickness at which it saturates to the bulk value. At $T=300 \mathrm{~K}$, the bulk resistivity of Pt is $\rho_{\mathrm{Pt}}^{300}=10.8 \pm 0.5 \mu \Omega \mathrm{cm}$ and $\lambda_{\mathrm{Pt}}^{300} \sim 3.74 \mathrm{~nm}$.

The thickness dependence of the resistivity of a thin film (or wire) is often studied using the "FS" model formulated some 70-80 years ago by Fuchs [50] and Sondheimer [51] and named after them, in which surface scattering is treated phenomenologically using Boltzmann transport theory. In the case of a monocrystalline "free-standing slab" with only bulk and surface scattering of charge carriers, the thickness dependent resistivity, $\rho$ is given by

$$
\begin{aligned}
\rho(p, d / \lambda)= & \rho_{b}\left[1-\frac{3}{2(d / \lambda)}(1-p)\right. \\
& \left.\int_{1}^{\infty}\left(\frac{1}{t^{3}}-\frac{1}{t^{5}}\right) \frac{1-e^{(d / \lambda) t}}{1-p e^{(d / \lambda) t}} d t\right]^{-1}
\end{aligned}
$$

where $d$ is the thickness of the slab and $\lambda$ and $\rho_{b}$ are, respectively, the mean free path and resistivity of the bulk material. The "specularity coefficient" $p$ is the fraction of electrons scattered elastically from the surface independent of their velocity 


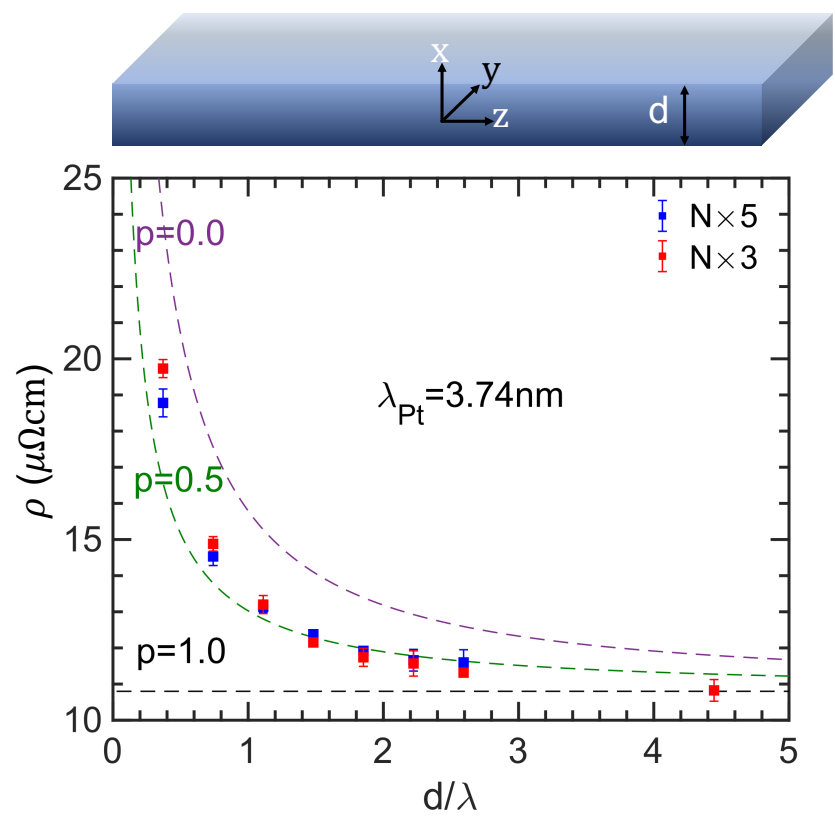

Figure 4.8: Average resistivity of Pt films calculated as a function of the effective thickness $d / \lambda$ where $d$ is the thickness of the film in the $x$ direction for transport in the $z(001)$ direction and $\lambda$ is the mean free path of bulk Pt at RT. The separation of periodically repeated thin films by vacuum ("vacuum thickness") is modelled using 5 layers of "empty" spheres in the $x$ direction. Calculations for each thickness are done for two cases with 5 (blue) and 3 (red) atomic layers repeated periodically in the $y$ direction. The dotted lines are calculated using the Fuchs-Sondheimer model $[50,51]$ for three different values of the specularity coefficient $p$ that describes the amount of completely diffusive surface scattering: completely specular $(p=1)$, partially specular $(p=0.5)$ and completely diffusive $(p=0)$. According to (4.20), choosing $p=1$ yields the bulk resistivity $\rho_{b}$ irrespective of $d / \lambda$.

and takes values ranging from 1 (specular) to 0 (diffusive). When all the electrons are reflected specularly from the surfaces, the resistivity is identical to that of the bulk; finite-size effects are not considered in the phenomenological FS electron gas model.

Values of $\rho$ calculated for different thicknesses of Pt at $T=300 \mathrm{~K}$ are plotted in Fig. 4.8 as a function of $d / \lambda$ where the thin-film enhancement of the resistivity is clear. The resistivity decays to within a few percent of its bulk value when $d \sim 4 \lambda$ and follows the FS model with $p \sim 0.5$ quite well even though the only surface roughness that is included is what results from thermal disorder. The number of layers $M$ in the $y$ direction has been limited to three throughout this chapter in order 


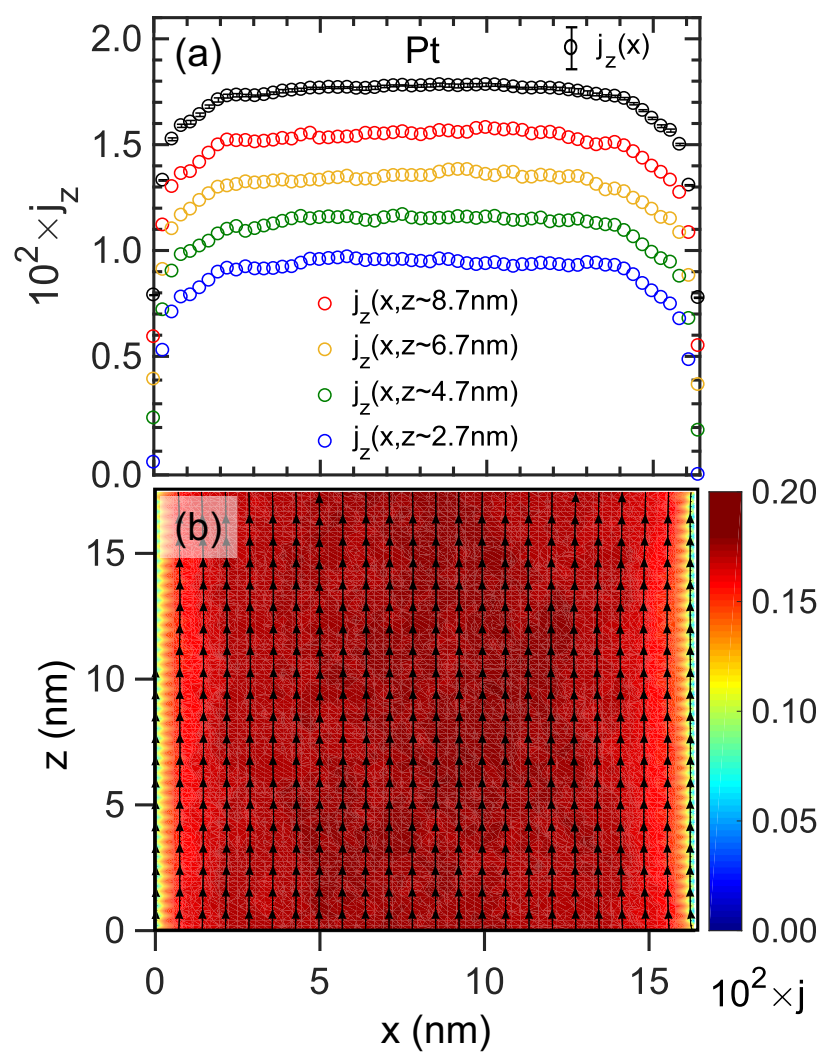

Figure 4.9: (a) Top curve, black symbols: current density $\bar{j}_{z}(x)$ obtained by averaging over $y$ and $z$. Colour symbols: $\bar{j}_{z}\left(x, z_{0}\right)$ obtained by averaging over $y$ and $z=z_{0} \pm 5$ layers in the $z$ direction for four different values of $z_{0}$ that are offset from the black curve in steps of $\Delta j=0.002$ for clarity. The error bars, that are smaller than the symbol size, indicate the average deviation over 10 random configurations of disorder. (b) Streamlines of the current vector $\mathbf{j}(x, z)=j_{x} \mathbf{i}+j_{z} \mathbf{k}$ in the $x z$ plane. The colour contour in the background corresponds to the magnitude of $\mathbf{j}(x, z)$.

to be able to study films with a reasonably large thickness given by the number of layers $N$ in the $x$ direction. In Fig. 4.8 this is shown to be a reasonable compromise for all but the thinnest of films where the results of calculations with $M=3$ and 5 are compared. The thickness dependent resistivity analysis confirms that $d / \lambda$ is the appropriate length scale for transport in slabs with finite thickness.

For $N=60$ atomic layers corresponding to a film thickness of $\sim 4.4 \lambda_{\mathrm{Pt}}^{300}$ and "bulk-like" behaviour, the charge current $\bar{j}_{z}(x)$ resulting from averaging over $y$ and $z$ is plotted as a function of $x$ in Fig. 4.9(a) (black symbols). The current density at different $z$ coordinates (coloured symbols) shows that $j_{z}$ is only weakly dependent on $z$ unlike what we saw in $\mathrm{Au}$. The reason is that $\lambda_{\mathrm{Pt}}^{300}$ is much shorter than the 
length of the scattering region. In Fig. 4.9(b), the current streamlines in the $x z$ plane are superimposed on a colour map of the magnitude $j(x, z) \equiv \sqrt{j_{x}^{2}+j_{z}^{2}}$ of $\mathbf{j}(x, z)$. Within the uncertainties of the calculation the current is constant except very close to the surface where a rapid decay in the current occurs over a length of $2 \mathrm{~nm} \sim \frac{1}{2} \lambda_{\mathrm{Pt}}^{300}$. A more detailed study of transport through thin Pt films will examine the effect of film orientation and surface roughness [202].

\section{Pt|Ir multilayer}

We study a Pt|Ir multilayer at an elevated temperature of $T=800 \mathrm{~K}$ chosen to make it possible to realise "bulk" like behaviour with scattering region sizes that are computationally tractable. Lattice disorder was introduced as described in Sec. 4.2 to reproduce the experimental bulk resistivities of $\mathrm{Pt}, \rho_{\mathrm{Pt}}^{800}=28.1 \pm 0.4 \mu \Omega \mathrm{cm}$ and of Ir, $\rho_{\mathrm{Ir}}^{800}=16.1 \pm 0.6 \mu \Omega \mathrm{cm}$ at $800 \mathrm{~K}$ [62] for which $\lambda_{\mathrm{Pt}}^{800} \sim 1.4 \mathrm{~nm}$ and $\lambda_{\mathrm{Ir}}^{800} \sim 2.4 \mathrm{~nm}$.

Ir and Pt are both fcc metals with an equilibrium lattice mismatch of only $2 \%$. We neglect this mismatch and use a common lattice constant of $a_{\mathrm{Pt}}=0.392 \mathrm{~nm}$ in the following. The scattering geometry is constructed as sketched in Fig. 4.5 with 60 (110) planes each of Pt and Ir stacked in the [110] $x$ direction. It consists of 90 (001) atomic layers sandwiched between ballistic Ir leads in the $z$ direction chosen to be the crystal [001] direction with periodicity of three atomic layers in the $y$ direction. A current is injected in the $z$ direction from the Ir leads, parallel to the Pt|Ir interface.

We average $j_{z}(x, y, z)$ over $y$ and $z$ and plot the resulting $\bar{j}_{z}(x)$ as a function of $x$ across the interface in Fig. 4.10(a). The horizontal black lines indicate averaged asymptotic values of current densities calculated separately for $x \in \operatorname{Pt}$ and $x \in \operatorname{Ir}$. A transition is clearly visible over a length scale of $\lambda_{i}$ about the interface. The asymmetry of the transition region with respect to the atomic interface simply reflects the difference between the mean free paths of the two materials. Within the error bars of our calculation the ratio of the equilibrium values of the currents averaged over the Ir and Pt volumes, $\bar{j}_{z}^{\mathrm{Ir}} / \bar{j}_{z}^{\mathrm{Pt}}=1.71$, mirrors the ratio $\rho_{\mathrm{Pt}}^{800} / \rho_{\mathrm{Ir}}^{800}=1.75$ confirming that bulk behaviour is recovered inside the slabs. As shown in Fig. 4.10(b) where the total charge currents in Pt and in Ir are plotted as a function of $z$, the current injected from the leads attains its asymptotic distribution essentially immediately.

In Fig. 4.10(c) we plot streamlines calculated for the charge current in a plane perpendicular to the interface in the Pt|Ir bilayer. Streamlines are parallel to the $z$ axis everywhere suggesting no current flow across the interface in the $x$ direction so each material can be treated as an independent transport channel. A colour map corresponding to the magnitude of the charge current $j=\sqrt{j_{x}^{2}+j_{z}^{2}}$ is shown in the background for reference. 


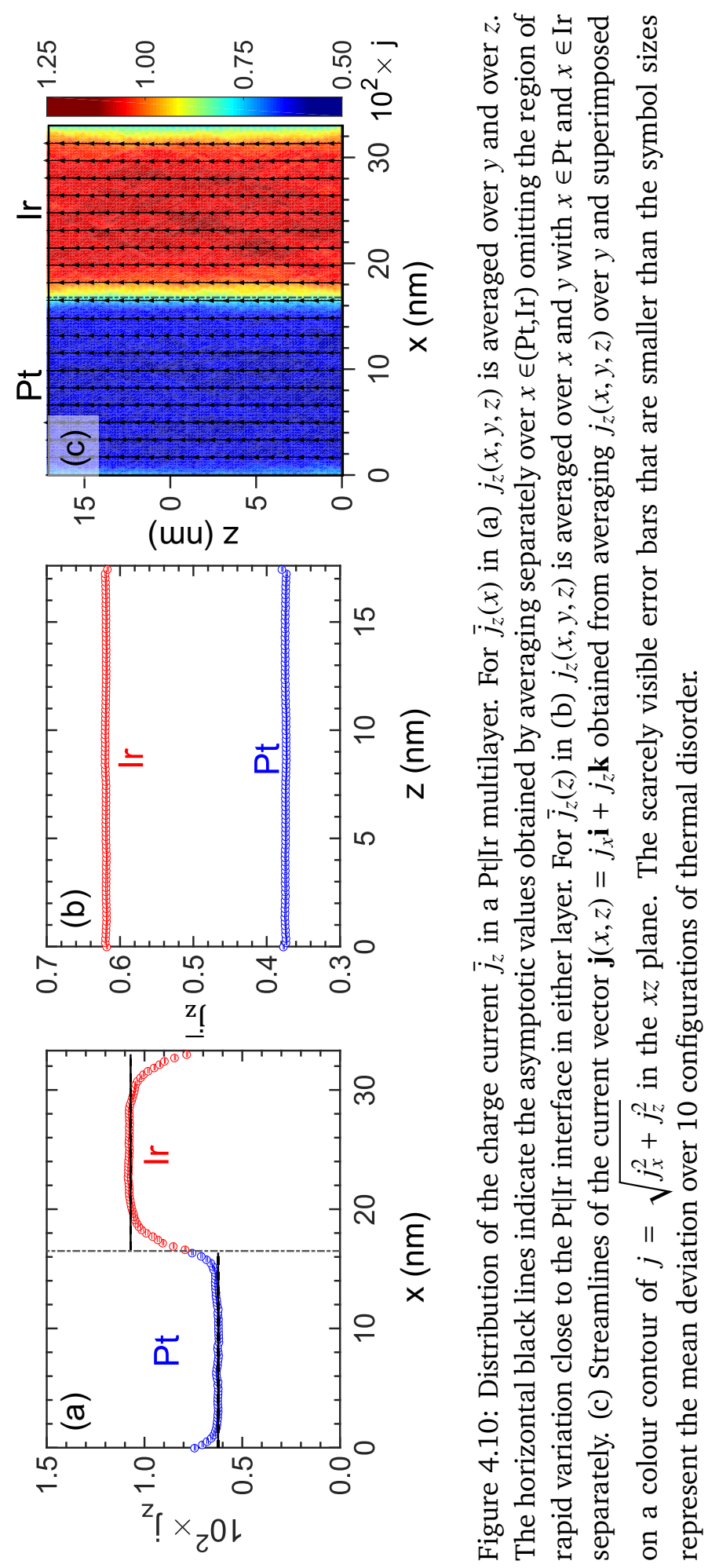




\subsubsection{Effect of different lead materials}

Perhaps surprisingly, neither Fig. 4.7 nor Fig. 4.10 provides any indication of current redistribution from the high resistivity to the low resistivity metal, the phenomenon know as shunting. It transpires that this is because these calculations were carried out using the lower resistivity material of the multilayer pair as the lead material so that there is essentially no contact resistance for the low resistivity channel. Because of the mismatch between their electronic structures, there is a substantial contact resistance between the high conductance lead and the high resistivity material. To lowest order, this contact resistance is the same as the interface resistance between the two materials making up the multilayer. While it is desirable that the results of calculations with the scattering formalism should be independent of the materials used for the leads, this is not always possible in practice because of present memory and computational time constraints. We illustrate this by considering what happens if we use the high resistivity material of the multilayer pair as lead material.

\section{$\mathrm{Au} \mid \mathrm{Pt}$}

Instead of using Au leads to inject a current into the Au|Pt multilayer, we now use Pt. In the Knudsen limit illustrated by the upper panels of Fig. 4.11, the current density does not saturate in the $x$ direction in either Pt or Au (Fig. 4.11(a)) nor does it saturate in the $z$ direction in either Pt or Au for the lengths of scattering region considered here (Fig. 4.11(b)). Compared to the results with Au leads, the current density in $\mathrm{Pt}$ is higher whereas that in $\mathrm{Au}$ is lower. Because the current does not saturate in the $z$ direction in Fig. 4.11(b), the $\bar{j}_{z}(z)$ plotted in Fig. 4.11(a) was obtained by averaging $j_{z}(x, y, z)$ over $y$ and over the ten central layers in the $z$ direction (layers 41 to 50 out of a total of 90). As a function of $x$, the anomalous dip at the interface in the current profile plotted in Fig. 4.11(a) is much larger than in Fig. 4.7(a) with Au leads.

In Fig. 4.11(b), the injection of charge carriers from Pt leads into Au (red symbols) is much lower than the injection from Au leads into Au (upper grey line). This can be attributed to the existence of an interface resistance between the ballistic Pt lead and diffusive Au. The converse applies for the injection into diffusive Pt from a ballistic Pt lead which is now higher (blue symbols) than when Au leads were used (lower grey line). Shunting tries to achieve the asymptotic situation where $\rho_{\mathrm{Pt}} \bar{j}_{z}^{\mathrm{Pt}}=\rho_{\mathrm{Au}} \bar{j}_{z}^{\mathrm{Au}}$ by diverting current from Pt to Au. We see this happening in Fig. 4.11(b) with $\bar{j}_{z}^{\mathrm{Au}}(z)$ increasing and $\bar{j}_{z}^{\mathrm{Pt}}(z)$ decreasing towards the centre of the scattering region. With our present computational resources, the number of layers in the $z$ direction required to achieve asymptotic behaviour is not tractable for the supercell sizes considered here.

The streamlines plotted in Fig. 4.11(c) now curve towards the Au layers of 


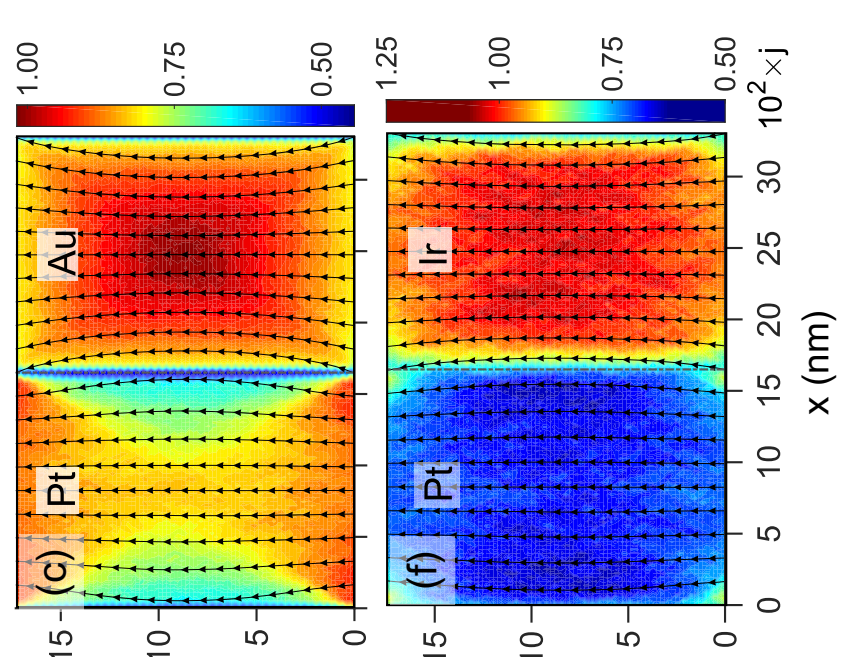

(uu) z
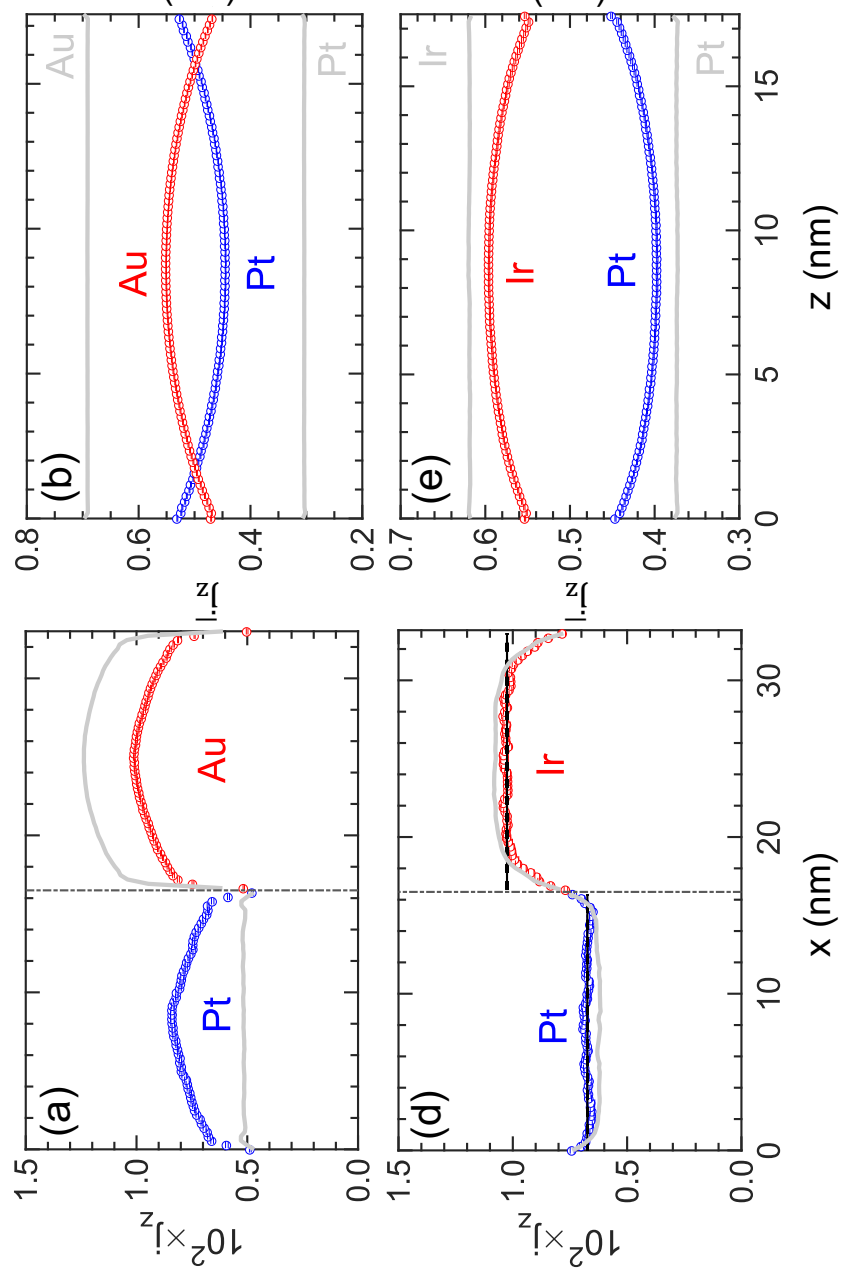

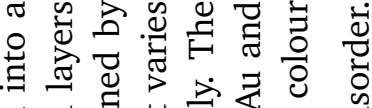

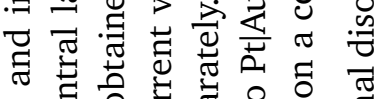

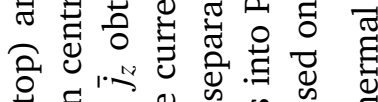

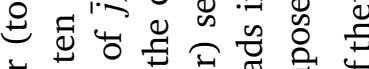

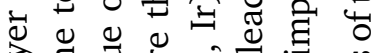

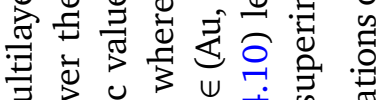

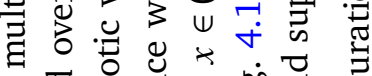

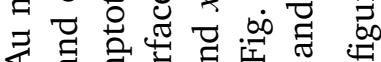

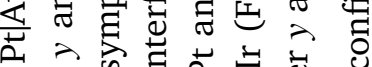

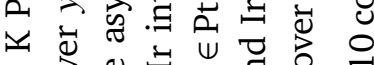

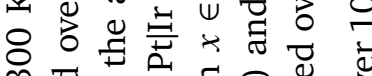
$m$ \&

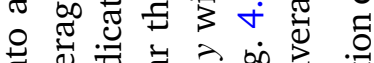

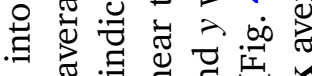

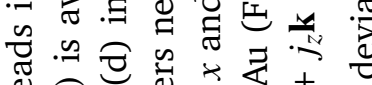
Q

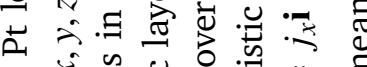

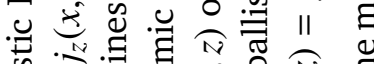

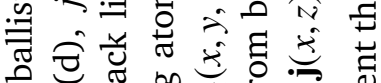
घ

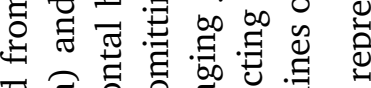

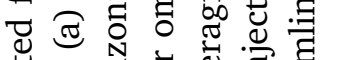

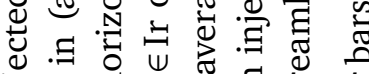

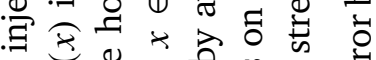

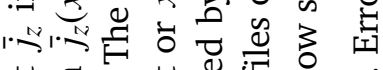

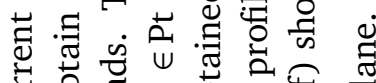
苞苛

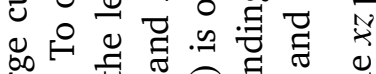

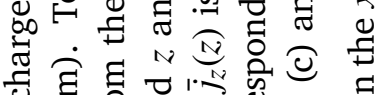

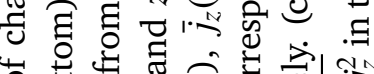

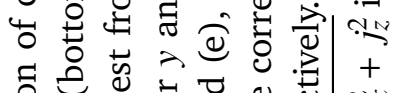
.

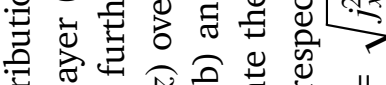

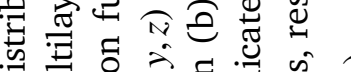

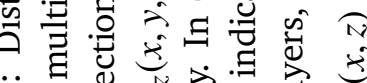

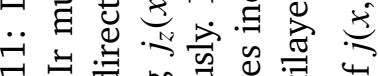

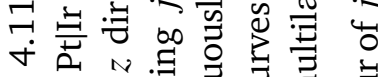
ฯ

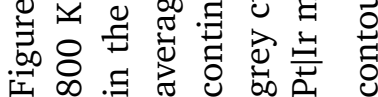


the multilayer indicating the flow of current across the interface from Pt into Au. Charge transport in the non-asymptotic case studied here is not amenable to description using a simple parallel resistance model following Ohm's law. It is worthwhile noting that for nanoscale experiments using heterostructures composed of metals with long mean free paths like $\mathrm{Cu}$, asymptotic current distributions cannot be guaranteed for short lengths and the application of semiclassical models may be contentious.

\section{Pt|Ir}

We now look at what happens when Pt leads are used in the classical limit for the $800 \mathrm{~K} \mathrm{Pt} \mid \mathrm{Ir}$ multilayer illustrated in the lower panes of Fig. 4.11. Comparing Fig. 4.11 (a) and (d), we see a striking difference between the classical and Knudsen limits near the interface. In Fig. 4.11(d) $j_{z}(x)$ varies gradually across the interface essentially interpolating between the saturated values calculated previously using Ir leads (indicated in grey) with the ratio of the mean values of the current density in each slab (black lines) $\bar{j}_{z}^{\mathrm{Ir}} / \bar{j}_{z}^{\mathrm{Pt}}=1.52$ falling short of the ratio $\rho_{\mathrm{Pt}}^{800} / \rho_{\mathrm{Ir}}^{800}=1.71$. As we saw for Pt|Au, shunting of the charge current is seen in Fig. 4.11(e) to vary along the transport direction $z$ but the crossover seen near the leads for Pt|Au is now absent. And just as we found for Pt|Au, the reduction in the current injected into Ir from Pt leads can be attributed to the interface resistance between ballistic $\mathrm{Pt}$ and diffusive Ir being larger than the negligible resistance between ballistic Pt and diffusive Pt. Streamlines of the current vector in Fig. 4.11(f) now curve into the Ir layer of the multilayer indicating a net flow from Pt into Ir.

The above examples show that longer scattering regions are needed in order to realize the situation where the currents reach their asymptotic distributions that are independent of the choice of lead material. This will become increasingly difficult as the temperature is lowered. The layers considered in this study are comparable in thickness to those use in many experiments in the field of spintronics where $\mathrm{Pt}$ layers are typically 10-20 nm thick and current distributions are expected to be asymptotic because of the longer lengths of samples. However, by using the high conductivity material in a multilayer as lead material, we showed that it is possible to probe the asymptotic state.

\subsection{Discussion}

We have presented a scheme to study spin and charge currents in nontrivial nanostructures containing surfaces and interfaces that builds upon an extremely efficient fully relativistic quantum mechanical scattering formalism [57, 153] and illustrated it with a study of charge transport in thin films and multilayers of nonmagnetic materials. The specific examples that we considered viz. Pt|Ir and Pt|Au 
multilayers as well as free-standing thin films of Pt and Au are illustrative of transport regimes where the mean free path $\lambda$ is either much larger than the thickness $d$ of individual layers or much smaller. The ratio $\lambda / d$ is the Knudsen number (Kn) that is well known from fluid physics. As pointed out early on by Fuchs [50], Sondheimer [51] and others [189], it plays a crucial role in determining how currents are distributed near a surface where diffusive scattering leads to a suppression of the current in a thin film as confirmed by numerous experimental studies as well as by our calculations for Au and Pt. The same current suppression is apparent in just the Au layer of a Au|Pt multilayer for which $\mathrm{Kn}>1$ but is much smaller in the Pt layer leading to a current density that varies nonmonotonically when we pass from the centre of the Pt layer through the interface to the centre of the Au layer. For a Pt|Ir multilayer at $800 \mathrm{~K}$ for which $\mathrm{Kn}<1$ there is a smooth and continuous variation of the current density through the interface.

Although the need to more accurately describe current distributions in metallic multilayers, including transient shunting effects, is widely recognized in order to interpret spin-transport experiments [52, 109, 203, 204], there has been little progress in devising improved methods of doing so [53]. At the same time, in the semiconductor world, there is a growing need to describe electron transport in wires whose size is being constantly reduced $[49,169]$ in order to identify improved interconnect materials. While we performed calculations for systems of $O\left(10^{4}\right)$ atoms, accessing the asymptotic limit at room temperature given by $\mathrm{Kn}>1$ for high conducting materials such as $\mathrm{Cu}$ with a long mean free path requires calculations on larger systems $\sim O\left(10^{5}\right)$ atoms. This is currently only limited by computer memory and computational time which will be met by the next generation of computers. In conclusion, our fully resolved current scheme makes it possible to accurately predict electronic transport in the complex geometries frequently encountered in modern microelectronics without introducing empirical parameters. 


\section{5 \\ Spin Hall Effect in a thin Pt film}

A density-functional-theory based relativistic scattering formalism is used to study charge transport through thin Pt films with room temperature lattice disorder. A Fuchs-Sondheimer specularity coefficient $p \sim 0.5$ is found to describe the charge current even in the absence of surface roughness. The spin Hall current perpendicular to the surface is poorly described using the model currently used to interpret experiments leading us to develop an improved model that takes surface effects into account. A reduced value of the spin Hall angle at the surface is needed to describe the first-principles transport results whose value depends on the film orientation. The in-plane spin Hall effect is substantially enhanced by the surface.

\subsection{Introduction}

The passage of a charge current through a heavy metal excites orthogonal spin currents because of the asymmetric spin scattering mediated by spin-orbit coupling, the well-known spin Hall effect (SHE) [12, 25, 73, 76, 77]. At an interface or surface which breaks the translational symmetry, spin accumulates on a length scale of the spin-flip diffusion length (SDL) $l_{\mathrm{sf}}$ [68]. For a thin film of finite thickness $d$ in the $x$ direction, see Fig. 5.1, the diffusive spin Hall current $j_{s y}^{x}(x)$ towards the surface at $x=d / 2$ that is generated in response to a charge current $j_{c}^{z}$ applied in the $z$ direction and the resulting spin accumulation $\mu_{s y}^{x}(x)$ are described by $[21,68]$

"Submitted as: R. S. Nair, M. Rang, P. J. Kelly, "Spin Hall Effect in a thin Pt film". 


$$
\begin{aligned}
& j_{s y}^{x}(x)=\Theta_{\mathrm{sH}} j_{c}^{z}\left[1-\frac{\cosh \left(x / l_{\mathrm{sf}}\right)}{\cosh \left(d / 2 l_{\mathrm{sf}}\right)}\right] \\
& \mu_{s y}^{x}(x)=2 \Theta_{\mathrm{sH}} j_{c}^{z} \rho l_{\mathrm{sf}}\left[\frac{\sinh \left(x / l_{\mathrm{sf}}\right)}{\cosh \left(d / 2 l_{\mathrm{sf}}\right)}\right] .
\end{aligned}
$$

Here, the spin current in the $x$ direction is polarized in the $y$ direction and the spin Hall angle (SHA) $\Theta_{\mathrm{sH}}$ characterizes the efficiency of charge-to-spin conversion. This model is expected to hold for $d>l_{\mathrm{sf}}$ and is used to extract values of $l_{\mathrm{sf}}$ and $\Theta_{\mathrm{sH}}$ from experiments on NM|FM bilayers comprising nonmagnetic (NM) and ferromagnetic (FM) metals using spin-pumping and the inverse spin Hall effect (ISHE) [75, 81, 82] or the SHE and spin transfer torque [84], or using the nonlocal spin injection method $[79,85]$. The NM metal that is most frequently studied is Pt whose thickness is typically chosen in the range $10-20 \mathrm{~nm}$. The values of $l_{\mathrm{sf}}$ and $\Theta_{\mathrm{sH}}$ reported for Pt span an order of magnitude $[18,77]$. All three experimental methods rely on interfaces to inject or detect the spin current and a failure to take account of interface spin-flipping was suggested [19] as the reason for the wide spread. However, taking it into account has not brought consensus any closer about the values of the SDL and SHA [20].

Recently the magneto-optical Kerr effect (MOKE) was used to probe the spin accumulation in a thin film and thereby estimate $\Theta_{\mathrm{sH}}$ and $l_{\mathrm{sf}}$ without introducing an interface between Pt and another metal [105]. However, the values of $\Theta_{\mathrm{sH}}$ and $l_{\mathrm{sf}}$ extracted from these experiments for Pt are twice those of the best available theoretical estimates using ab-initio calculations $[20,93]$ motivating us to examine the SHE in thin films in more detail.

\subsection{Method}

To study transport in a thin film, we generalized a scheme for calculating layerresolved currents [20] from the solutions of fully relativistic quantum mechanical scattering calculations $[57,153]$ to allow full atomic resolution [205]. The geometry used to study thin film transport is illustrated in Fig. 5.1 for a (110) surface perpendicular to the $x$ direction. Periodic boundary conditions are used in the $x$ and $y$ directions whereby use can be made of Bloch's theorem to efficiently determine and keep track of the lead states [16]. Vacuum is represented in the atomic spheres approximation (ASA) using "empty spheres" [198-200]. Spin currents are calculated as in Ref. [20] and their interpolation onto a three dimensional grid is described in chapter 4 


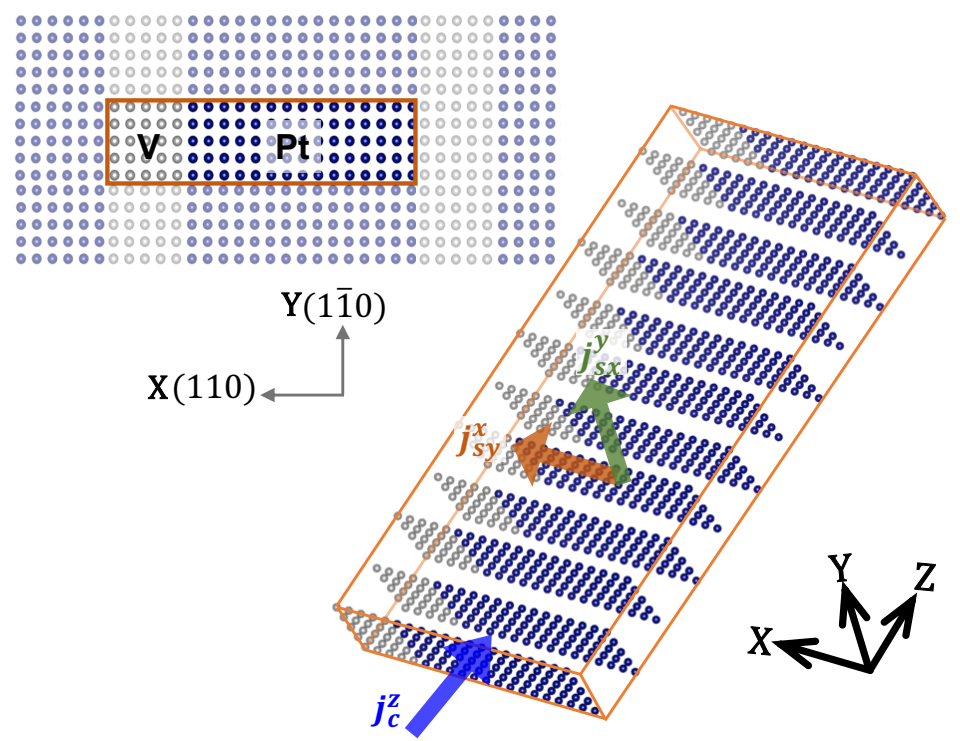

Figure 5.1: Schematic of the geometry used to study transport in a [110] oriented thin film of fcc Pt (blue). The layer separation in the $z$ direction is exaggerated for clarity. The vacuum region is modelled using a thickness of vacuum equal to five layers of "empty" spheres (grey) in the $x$ direction and superlattice periodicity in the $x$ and $y$ directions is assumed. Three unit cells in the $y$ direction and a few atomic layers of the repeated unit cells in the $\pm x$ direction are shown on the top left for a single [001] plane. A charge current $j_{c}^{z}$ is passed between leads in the $+z$ and $-z$ directions (not shown) exciting spin currents $j_{s y}^{x}$ in the $x$ direction which accumulates at the surface (orange arrow) and $j_{s x}^{y}$ flowing in the $y$ direction parallel to the surface.

\subsection{Calculations}

The results we find for the spin Hall current $j_{s y}^{x}(x)$ flowing towards the surface of the thin film sketched in Fig. 5.1 are presented in Fig. 5.2 for a free standing Pt slab of thickness $d=31.60 \mathrm{~nm}$ corresponding to 115 atomic layers in the $x$ direction that are separated from their periodic images by five layers of empty spheres. 90 layers of this $360=(115+5) \times 3$ supercell were stacked in the $z$ direction and the atoms in the scattering region were displaced at random with a Gaussian distribution of atomic displacements chosen to reproduce the experimental bulk resistivity of $\rho=10.8 \mu \Omega \mathrm{cm}$ [62] at room temperature (RT $=300 \mathrm{~K}$ ). The thickness of the slab is $d \approx 6 l_{\text {sf }}$ for a value of $l_{\mathrm{sf}} \approx 5.2 \mathrm{~nm}$ estimated from the decay of a fully polarized spin current injected into Pt [20,93] making it essentially bulk-like. Consistent with this is that at the center of the slab, the spin Hall angle (the ratio of the transverse spin current measured in units of $\hbar / 2$ to the longitudinal charge 

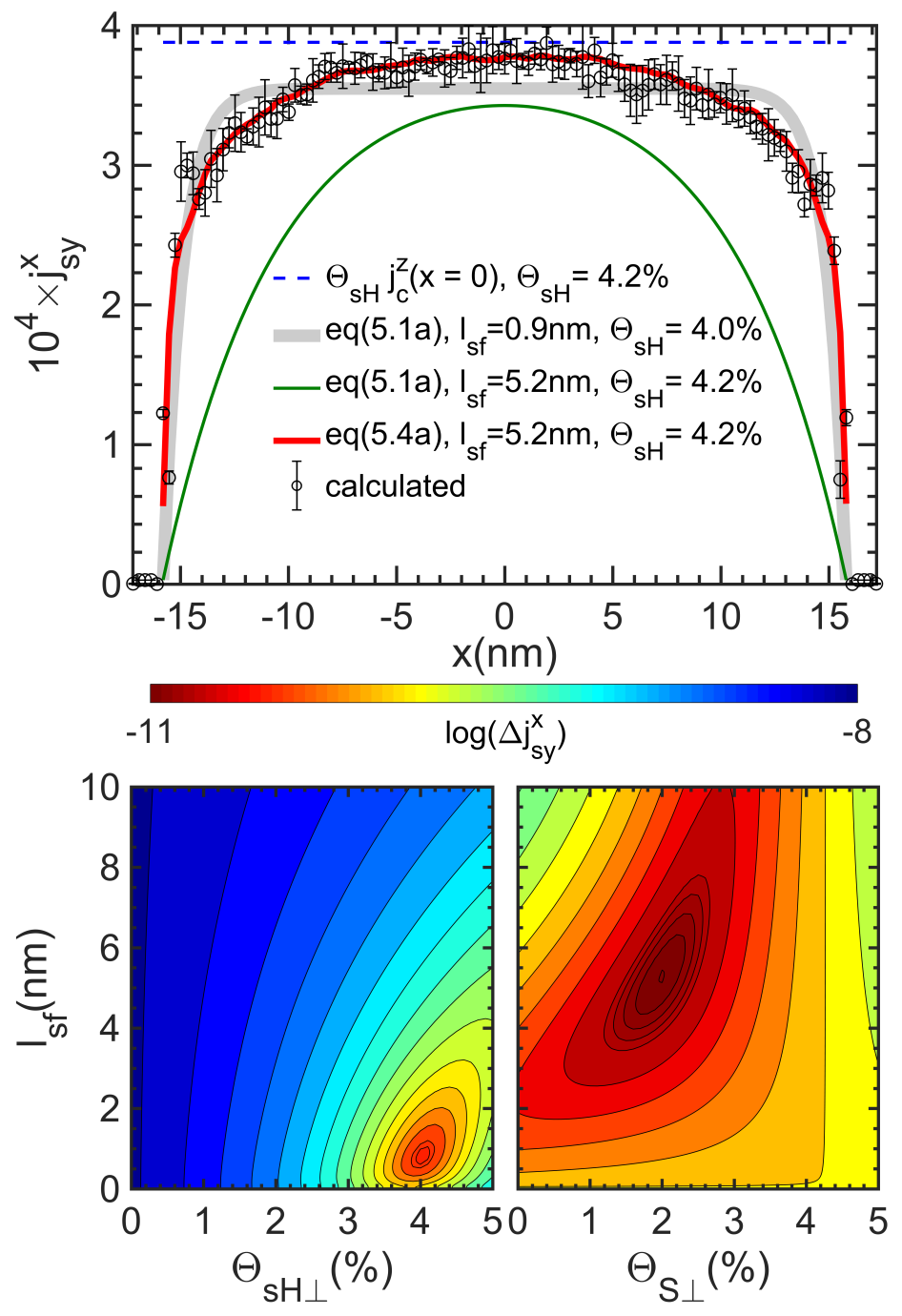

Figure 5.2: (Top) Spin current $j_{s y}^{x}$ flowing in the $x$ direction at room temperature as a function of $x$ (circles). The green curve results from equation (5.1a) using the "bulk" values $l_{\mathrm{sf}}=5.2 \mathrm{~nm}$ and $\Theta_{\mathrm{sH}}=4.2 \%$ calculated for bulk Pt $[20,93]$. The grey curve is the best fit using equation (5.1a) with $l_{\mathrm{sf}}=0.9 \mathrm{~nm}$ and $\Theta_{\mathrm{Pt}}=$ $4.0 \%$. The red curve is obtained by taking the calculated $j_{c}^{z}(x)$, bulk values of $l_{\mathrm{sf}}=$ $5.2 \mathrm{~nm}$ and $\Theta_{\mathrm{Pt}}=4.2 \%$ and fitting with (5.4a) and (5.5) to obtain $\Theta_{\mathrm{S}}=2.54 \pm$ $0.03 \%$. The horizontal dashed blue line represents the bulk value $\Theta_{\mathrm{Pt}}=4.2 \%$ for the charge current density at the centre of the thin film. Error bars on the calculated data represent the average deviation over five configurations of thermal disorder. (Bottom) Logarithm of the root mean square residuals using (left) equation (5.1a) to describe the ab-initio calculated data in the $\left\{l_{\mathrm{sf}}, \Theta_{\mathrm{Pt}}\right\}$ parameter space and (right) equations (5.4a) and (5.5) in the $\left\{l_{\mathrm{s}}, \Theta_{\mathrm{S}}\right\}$ parameter space with the bulk value of $\Theta_{\mathrm{Pt}}=4.2 \%$. 
current measured in units of the electron charge $-e$ ) is the bulk value, $\Theta_{\mathrm{Pt}} \equiv \Theta_{\mathrm{sH}}^{\mathrm{Pt}} \approx$ $4.2 \%$ [20]. Small oscillations that are discernible in the calculated profiles are attributed to standing waves that are not described by semi-classical (Boltzmann) descriptions of transport. Using the bulk values of $l_{\mathrm{sf}}$ and $\Theta_{\mathrm{sH}}$ in (5.1a) yields the green curve in Fig. 5.2 that is clearly a poor representation of the ab-initio results. If instead we perform a least squares fit in the $\left(l_{\mathrm{sf}}, \Theta_{\mathrm{S}}\right)$ parameter space (bottom left panel) we find a best fit with $l_{\mathrm{sf}}=0.9 \mathrm{~nm}$ and $\Theta_{\mathrm{Pt}}=4.0 \%$ yielding the grey curve in Fig. 5.2 (top panel). The extremely short value of $l_{\mathrm{sf}}$ and the shape of the corresponding best fit curve differ quite substantially from the calculations. To understand the discrepancy, we generalize the Valet-Fert (VF) model of Zhang [68] to include surface spin-flipping and a surface SHE.

\subsection{Improved Valet-Fert model}

To include surface effects, the thin film geometry is modelled as a trilayer with two surface (S) regions of finite thickness $t$ sandwiching the non-magnetic "bulk" metal (NM) of thickness $d$ in the $x$ direction as shown schematically in Fig. 5.3(a). For each layer $i(=\mathrm{S}, \mathrm{NM})$, the transverse spin current density and spin accumulation excited by a longitudinal charge current density $j_{c}^{z}(x)$ in the $z$ direction are

$$
\begin{aligned}
& j_{s y}^{x}(x)=\frac{1}{2 e \rho_{i} l_{i}}\left[A_{i} e^{-x / l_{i}}-B_{i} e^{x / l_{i}}\right]+\Theta_{i} j_{c}^{z}(x) \\
& \mu_{s y}^{x}(x)=A_{i} e^{-x / l_{i}}+B_{i} e^{x / l_{i}}
\end{aligned}
$$

where $l_{i} \equiv l_{\text {sf }}^{i}$ describes the decay of a spin current in the layer $i, \rho_{i}$ is its resistivity and $\Theta_{i} \equiv \Theta_{\mathrm{sH}}^{i}$ its spin Hall angle. The metallic layer has inversion symmetry and the spin current and spin accumulation are continuous across the $\mathrm{NM} \mid \mathrm{S}$ interface. The surface layer is anisotropic and all three transport properties are considered to be tensors with the independent elements $\rho_{\mathrm{S}}^{\perp}, \rho_{\mathrm{S}}^{\|}, l_{\mathrm{S}}^{\perp}, l_{\mathrm{S}}^{\|}, \Theta_{\mathrm{S}}^{\perp}, \Theta_{\mathrm{S}}^{\|}$. Since we are interested in spin diffusion perpendicular to the surface, $l_{\mathrm{S}}^{\|}$and $\Theta_{\mathrm{S}}^{\|}$do not enter (5.2) while $\rho_{\mathrm{S}}^{\|}$is implicit in $j_{c}^{z}(x)$. We are left with three parameters $\rho_{\mathrm{S}}^{\perp}, l_{\mathrm{S}}^{\perp}$ and $\Theta_{\mathrm{S}}^{\perp}$ governing transport perpendicular to the surface in the finite surface layer $S$ that cannot be determined by fitting equations (5.2) for $j_{s y}^{x}$ and $\mu_{s y}^{x}$. Instead, taking the limit $t \rightarrow 0$ leads to the surface areal resistance $A R_{\mathrm{S}}$ and spin-memory loss (SML) parameter $\delta$ defined as

$$
\lim _{t \rightarrow 0} \rho_{\mathrm{S}}^{\perp} t=A R_{\mathrm{S}} \quad \text { and } \quad \lim _{t \rightarrow 0} t / l_{\mathrm{S}}^{\perp}=\delta
$$

where $\delta$ describes the spin current discontinuity that arises when $t \rightarrow 0$ and the first term on the right-hand side of $(5.4 \mathrm{a})$ is proportional to $\delta / A R_{\mathrm{S}}$. Since there is no transport across the surface into the vacuum we set $A R_{\mathrm{S}} \rightarrow \infty$ so the first term in (5.4a) vanishes giving rise to the boundary condition $j_{s y}^{x}=\Theta_{\mathrm{S}}^{\perp} j_{c}^{z}[ \pm(d / 2)]$ 


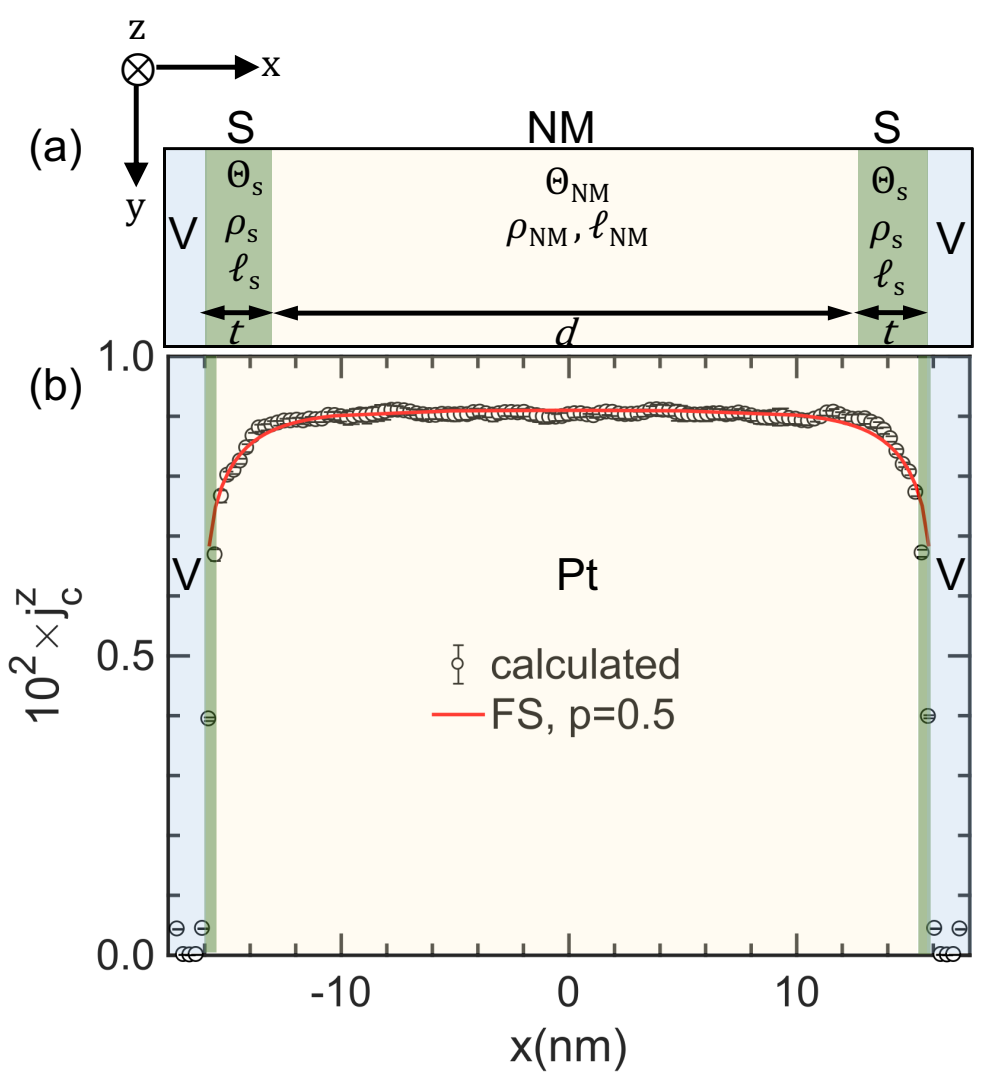

Figure 5.3: (a) Schematic of the trilayer geometry with a nonmagnetic (NM) slab sandwiched between two surface layers (S) of finite thickness $t$ that separate it from the vacuum (V). Spin transport in each layer $i$ is characterized by its bulk properties $\Theta_{i}, l_{i}$ and $\rho_{i}$. For the surface layer, these transform into surface quantities in the limit $t \rightarrow 0$. (b) Profile of the charge current $j_{c}^{z}(x)$ flowing through Pt in the $z$ direction averaged in the $y$ direction and sufficiently far from the leads in the $z$ direction as to be independent of $z$. The red curve is a fit using Fuchs-Sondheimer theory (FS) in the explicit form given in Ref. [189]. Error bars represent the average deviation over five configurations of disorder.

at $x= \pm(d / 2)$ compared to $j_{s y}^{x}=0$ in (5.1a). We use this condition and inversion symmetry in the $x$ direction to eliminate the coefficients $A_{i}$ and $B_{i}$ for the slab.

Introducing a surface region whose transport properties differ from those of the bulk poses the question as to how the longitudinal charge transport is affected by the surface. In Fig. 5.3(b), $j_{c}^{z}(x)$ is seen to deviate in the topmost 10 atomic layers from a constant bulk value and can be fit essentially perfectly using the FuchsSondheimer (FS) theory with the RT bulk resistivity $\rho_{\mathrm{Pt}}$, the mean-free-path $\lambda_{\mathrm{Pt}}=$ $3.74 \mathrm{~nm}$ estimated in the relaxation time approximation with the Fermi velocity 
averaged over the Fermi surface [93], and a specularity coefficient $p \sim 0.5$ [50, $51,189]$ without needing to explicitly invoke the surface transport parameter $\rho_{\mathrm{S}}^{\|}$. Using this $j_{c}^{z}(x)$ as the longitudinal charge current driving the spin-Hall current in (5.4a), we arrive at the following expressions describing spin diffusion in the presence of the spin Hall effect in a thin film

$$
\begin{aligned}
& j_{s y}^{x}(x)=-C_{\mathrm{S}} \cosh \left(x / l_{\mathrm{Pt}}\right)+\Theta_{\mathrm{Pt}} j_{c}^{z}(x) \\
& \mu_{s y}^{x}(x)=2 e \rho l_{\mathrm{Pt}} C_{\mathrm{S}} \sinh \left(x / l_{\mathrm{Pt}}\right)
\end{aligned}
$$

where the coefficient

$$
C_{\mathrm{S}}=\frac{\left(\Theta_{\mathrm{Pt}}-\Theta_{\mathrm{S}}^{\perp}\right) j_{c}^{\mathrm{z}}(|d / 2|)}{\cosh \left(d / 2 l_{\mathrm{Pt}}\right)}
$$

is clearly a surface dependent quantity and

$$
\Theta_{\mathrm{S}}^{\perp}=\frac{j_{s y}^{x}(|d / 2|)}{j_{c}^{z}(|d / 2|)} .
$$

For the [110] surface we find a value of $\Theta_{\mathrm{S}}^{\perp}=2.5 \%$, substantially lower than the bulk value. This result as well as those for [001] and [111] oriented films are given in Table 5.1. For the very smooth [111] surface we also considered a "rough" variant prepared by randomly removing half of the atoms from the topmost layer. The results obtained from equation (5.6) are seen to exhibit a very strong dependence on the surface. This comes about not because of a strong dependence of $j_{s y}^{x}(x)$ on the surface but because of (i) the FS suppression of $j_{c}^{z}(x)$ close to the surface and (ii) the connectivity of the atoms making up the surface layer. For example, $j_{c}^{z}(|d / 2|)$ has a large value for the smooth [111] surface where every surface atom has six neighbouring surface atoms and it is this which leads to a large value of $j_{c}^{z}(|d / 2|)$ and low value of $\Theta_{\mathrm{S}}^{\perp}$.

Just as the resistivity is not a local property on the length scale of the mean free path, there is no reason to describe the SHE as a local property as is done by the semiclassical VF description. At the centre of the thin film furthest from the surfaces, the absolute value of the leading, diffusive term in (5.4a) has its minimum value and $j_{s y}^{x}(x=0) / j_{c}^{z}(x=0) \approx \Theta_{\mathrm{sH}}$ as indicated by the dashed blue reference line in Fig. 5.2 corresponding to the bulk value of $\Theta_{\mathrm{Pt}}=4.2 \%$ [20]. Fixing $\Theta_{\mathrm{Pt}}$ at this bulk value, we scan the $\left\{l_{\mathrm{sf}}, \Theta_{\mathrm{S}}^{\perp}\right\}$ parameter space with (5.4a) and find a minimum occurs for values of $l_{\mathrm{sf}}$ in the range 4-6 nm and of $\Theta_{\mathrm{S}}^{\perp}$ in the range $1.5-2.5 \%$. Alternatively, we can fix $\Theta_{\mathrm{Pt}}$ and $l_{\mathrm{sf}}$ at their bulk values $\{4.2 \%, 5.2 \mathrm{~nm}\}$ and optimize the fit of (5.4a) and (5.5) to the ab-initio values of $j_{s y}^{x}(x)$ using the remaining free parameter $\Theta_{S}^{\perp}$. The result of doing so for the [110] surface is the red curve in Fig. 5.2 for a value of $\Theta_{S}^{\perp}=2.54 \pm 0.03 \%$. The fit is seen to be excellent. Values of $\Theta_{\mathrm{S}}^{\perp}$ for the other surfaces are given in Table 5.1 where a suppression of the surface SHA is seen for all orientations. 
Table 5.1: Room temperature transport parameters for differently oriented Pt thin films: specularity coefficient $p$; spin Hall angle in percent for spin-current perpendicular $\left(\Theta_{\mathrm{S}}^{\perp}\right)$ and parallel $\left(\Theta_{\mathrm{S}}^{\|}\right)$to the surface. Values of $\Theta_{\mathrm{S}}^{\perp}$ were obtained by fitting $j_{s y}^{x}(x)$ with (5.4a) and (5.5) with $\Theta_{\mathrm{Pt}}$ and $l_{\text {sf }}$ fixed at their bulk values. For the rough [111] calculations, $50 \%$ of the atoms in the surface layers were removed at random.

\begin{tabular}{lccccc}
\hline \hline & & \multicolumn{3}{c}{ Clean } & Rough \\
\cline { 3 - 5 } & & {$[110]$} & {$[100]$} & {$[111]$} & {$[111]$} \\
\hline$p$ & & 0.5 & 0.5 & 0.4 & 0.2 \\
$\Theta_{\mathrm{S}}^{\perp}(\%)$ & Eq.(5.6) & 2.0 & 1.7 & 0.1 & 3.1 \\
& Eqs.(5.4a)\&(5.5) & 2.1 & 3.8 & 3.5 & 2.0 \\
$\Theta_{\mathrm{S}}^{\|}(\%)$ & & 8.8 & 7.0 & 7.7 & 6.3 \\
\hline \hline
\end{tabular}

\subsection{Surface enhancement}

We have seen that $j_{c}^{z}$ and $j_{s y}^{x}$ depend strongly on the distance $x$ to a surface in a thin film. In Fig. 5.4 we plot the spin current that flows parallel to the surface, $j_{s x}^{y}$, and see that it also depends quite strongly on $x$. Unlike $j_{s y}^{x}(x)$ that changes on a length scale of $l_{\mathrm{sf}}, j_{s x}^{y}$ is essentially constant up to a mean-free-path of the surface where it increases to reach $\sim 150 \%$ of its mean value at the centre of the slab in spite of the FS suppression of the driving charge current $j_{c}^{z}(x)$ shown in Fig. 5.3. Thus in a sample that is finite in the $x$ and $y$ directions, see Fig. 5.1, a spin Hall current diffusing towards a surface or an interface will have an additional contribution from the surfaces parallel to the direction of diffusion.

To extract a surface SHA, we integrate $\Theta^{\|}(x)=j_{s x}^{y}(x) / j_{c}^{z}(x)$ from a position in the vacuum where all currents vanish, through the surface at $x=-d / 2$, in to $x$ and plot the result as a function of $x$ in the inset to Fig. 5.4. Using a linear fit we identify a surface region of thickness $t \sim 1.94 \mathrm{~nm}$ between where the integrated curve deviates from the linear bulk contribution and where it vanishes. We define the ratio of the corresponding intercept on the $y$ axis (red asterisk in Fig. 5.4) to $t$ as the surface contribution $\Theta_{\mathrm{S}}^{\|}$. For the [110] thin film, this ratio yields $\Theta_{\mathrm{S}}^{\|} \approx 8.8 \%$. Results for the other orientations given in Table 5.1 are all enhanced compared to the bulk value of $\Theta_{\mathrm{Pt}}$. 


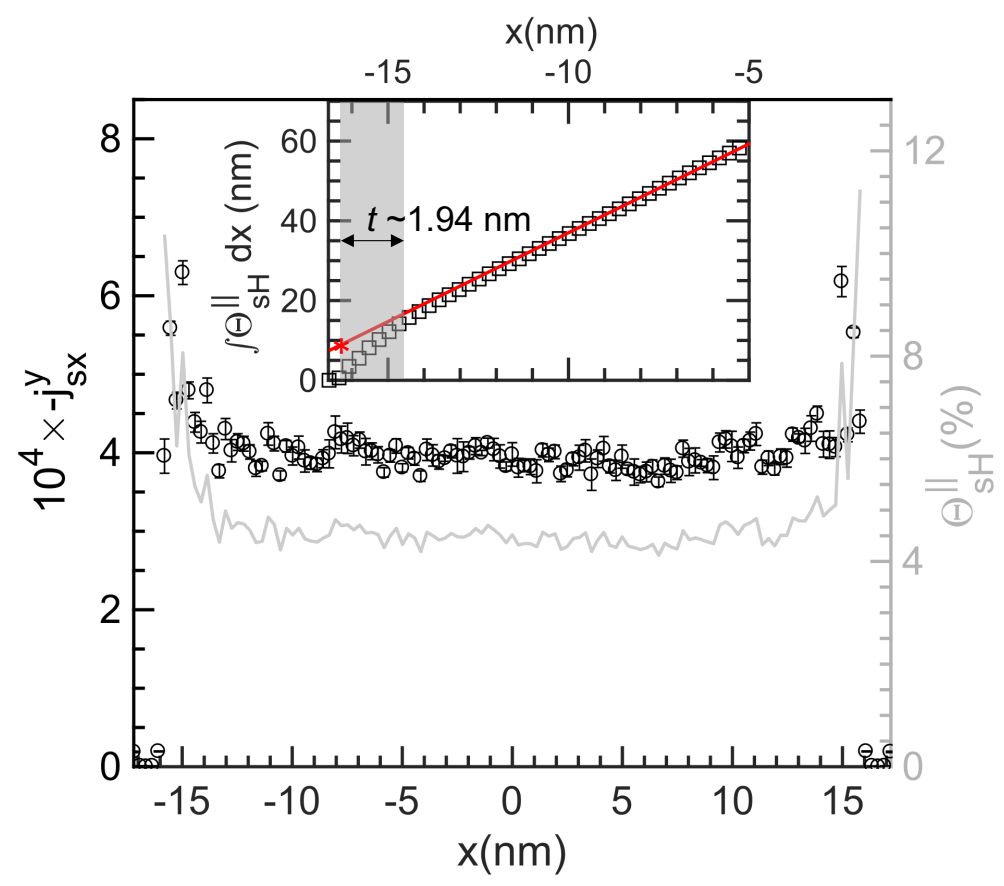

Figure 5.4: RT spin current $-j_{s x}^{y}$ propagating in the $y$ direction as a function of $x$ (left-hand axis). Error bars represent the average deviation across 5 configurations of disorder. The local ratio $-j_{s x}^{y} / j_{c}^{z} \equiv \Theta_{\mathrm{sH}}^{\|}$plotted as a function of $x$ is referenced to the right-hand axis. Inset: Integral of $\Theta_{\mathrm{sH}}^{\|}$from the centre of the vacuum region near the bottom surface to $x=-5 \mathrm{~nm}$, plotted as a function of $x$. The linear fit is shown in red and the "effective surface region" in grey. The value of the linear fit at the $x$ coordinate where $\int \Theta_{\mathrm{sH}}^{\|} d x$ becomes positive is indicated by a red asterisk.

\subsection{Discussion}

When allowance is made for a variation of the SHA at a surface, the spin-current profiles that we calculate from first-principles scattering theory are consistent with bulk values of the SHA and SDL that we calculated previously [20,93]. We find no evidence for a recently reported value of $l_{\mathrm{sf}}=11 \pm 3 \mathrm{~nm}$ obtained from MOKE observations of the spin accumulation induced in thin films by the SHE [105]. The asymptotic "bulk" resistivity of $20.6 \mu \Omega \mathrm{cm}$ that Stamm et al. report for thick films [105] is twice the handbook value quoted for RT Pt [62]. Although polycrystallinity and grain boundary scattering are frequently invoked $[162,190]$ to account for the increased resistivity of thin films [163-167] and wires [166, 168, 169], it is not known what effect these might have on the SHE-induced spin accumulation nor 
is any structural characterization reported that might account for the increased resistivity [105]. From the Elliott-Yafet relation $\rho l_{\mathrm{sf}}=$ const., one might expect $l_{\mathrm{sf}}$ to be shorter when the resistivity is high rather than longer.

\subsection{Summary}

First-principles calculations were used to examine the diffusion of the intrinsic spin Hall current perpendicular to the surface of a thin film of Pt at room temperature when a charge current is passed through the thin film. A marked suppression of the charge current parallel to the surface is found to be well reproduced by a phenomenological model due to Fuchs and Sondheimer and leads to a reduction of the spin source. A model of spin diffusion that is widely used to interpret experimental SHE studies fails to reproduce the spin current profiles that we calculate leading us to develop a more general model that provides an accurate description of the calculated spin current. A substantial enhancement of the spin Hall current parallel to the surface is predicted. 


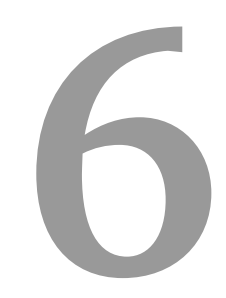

\section{Transverse spin injection in bilayers"}

We use first-principles scattering calculations and a recently developed discrete current scheme to study the spin currents driven transversely into non-magnetic $\mathrm{Au}$ (or $\mathrm{Cu}$ ) layers by the spin Hall effect in adjacent Pt (Ni or permalloy, Py) layers. The spin currents are excited by charge currents passed parallel to the interfaces of $A u|P t, P y| C u$ and $\mathrm{Ni} \mid \mathrm{Cu}$ periodic bilayers and are injected across the Py|Cu and Pt|Au interfaces with an efficiency of $\sim 40 \%$ and $\sim 60 \%$, respectively. A Valet-Fert scheme developed to describe the spin injection into Au from Pt yields a value for the spin memory loss parameter $\delta \sim 0.6$ and spin Hall angle $\Theta_{\mathrm{I}} \sim 3 \%$ for the Pt|Au interface at room temperature. Unlike Pt|Au, the spin injection in the FM|NM systems comes largely from the interface.

\subsection{Introduction}

Passing a charge current through a material like platinum with strong spin-orbit coupling results in a spin Hall effect (SHE) whereby a transverse spin current is generated with orthogonal spin and linear momentum directions $[12,73,206]$. Onsager reciprocity demands an inverse effect (ISHE) with the generation of a charge current orthogonal to a spin current pumped into the material and to its spin direction. It is the ISHE which is most frequently used in experiments to extract material dependent spin transport properties like the spin Hall angle $\Theta_{\mathrm{sH}}$ and the spin flip diffusion length $l_{\text {sf }}[103,207-209]$. The SHE has been theoretically studied using semi-classical drift-diffusion models [68], the Kubo [35, 100, 101, 108, 210] and

\footnotetext{
"In preparation: R. S. Nair and P. J. Kelly, "Transverse spin injection in bilayers".
} 
scattering [20, 34, 93] formalisms. Theoretical studies concerned with calculating the material dependence of the spin Hall conductivity (SHC) have focussed on bulk properties to exploit periodicity in lateral directions [93, 108, 210]. However, whether the ISHE is used to detect the spin current pumped by the precessing magnetization of a ferromagnetic material (FM) into an adjacent nonmagnetic material (NM) $[75,81-83]$ or the passage of a charge current through a NM material generates a spin current by the SHE that then exerts a (spin-transfer) torque (STT) on an adjacent FM material [84], experimental determinations of the SHC always involve interfaces. Use of the nonlocal spin-injection method $[79,85]$ involves two interfaces: an $\mathrm{FM} \mid \mathrm{NM}$ interface to create a spin accumulation and an $\mathrm{NM} \mid \mathrm{NM}^{\prime}$ interface to detect it. The recent use of MOKE experiments [105] to detect the spin accumulation at the surface of a material by probing it with polarized light also involves an interface, an $\mathrm{NM} \mid$ vacuum interface, i.e. a surface. These interfaces introduce additional parameters $[89,211]$ such as the spin memory loss (SML) parameter [48] or interface SHE [34] that are not accounted for adequately. Moreover, how a spin Hall current generated in a bulk FM material diffuses across a FM|NM interface when the magnetization $\mathbf{M}$ of the FM is not aligned with the propagating spins is not known. The only theoretical work we are aware of that considers the injection of spin Hall currents in bilayers deals with 2D model systems [212, 213]. This leads us to pose the question as to how efficiently a spin Hall current generated in a real material like Pt is injected into another nonmagnetic material like $\mathrm{Au}$. Addressing this question requires looking at the spatial distribution of intrinsic spin Hall currents in realistic bilayers. In this paper, we will do so for $\mathrm{NM} \mid \mathrm{NM}^{\prime}$ and FM|NM bilayers composed of materials with similar lattice constants, in particular $\mathrm{Pt}|\mathrm{Au}, \mathrm{Py}| \mathrm{Cu}$ and $\mathrm{Ni} \mid \mathrm{Cu}$. Pt, Py and Ni exhibit strong spin Hall effects while Au and $\mathrm{Cu}$ have long spin flip diffusion lengths and negligible intrinsic spin Hall angles.

This chapter is organized as follows. The main features of the quantum mechanical scattering calculations used in this work are sketched in Sec. 6.2 and some details of the computational parameters are given. A Valet-Fert [21] model that will be used to interpret the numerical spin injection results in $\mathrm{NM} \mid \mathrm{NM}^{\prime}$ is derived in Sec. 6.3. In Sec. 6.4 we calculate spin currents in periodically repeated bilayers starting with Pt|Au and apply our Valet-Fert (VF) model to extract interface parameters. We further discuss our calculations in $\mathrm{Py}(\mathrm{Ni}) \mid \mathrm{Cu}$ bilayers and present the results. Some conclusions are drawn in Sec. 6.5.

\subsection{Method}

To address how the transverse spin current excited by a longitudinal charge current is injected into an adjacent non-magnetic material, we use the results of relativistic quantum mechanical scattering calculations $[57,153]$ to construct inter-atomic charge $\left(j_{c}\right)$ and spin $\left(j_{s}\right)$ currents [20] which are interpolated onto a three di- 
mensional grid yielding $j(x, y, z)$ [93]. A 3D sketch of the scattering region $(\mathcal{S})$ sandwiched between left $(\mathcal{L})$ and right $(\mathcal{R})$ leads used to study an fcc Au|Pt bilayer is shown in Fig. 6.1. Note that there is still superlattice periodicity in the $x$ and $y$ directions. A charge current is injected from a lead in the [001] $z \equiv Z$ direction $\left(j_{c}^{z}\right)$ leading to spin currents in the $x$ [110] and $y$ [11 0 ] directions as schematically shown in Fig. 6.1. Details of the scheme can be found in Ref. [205].

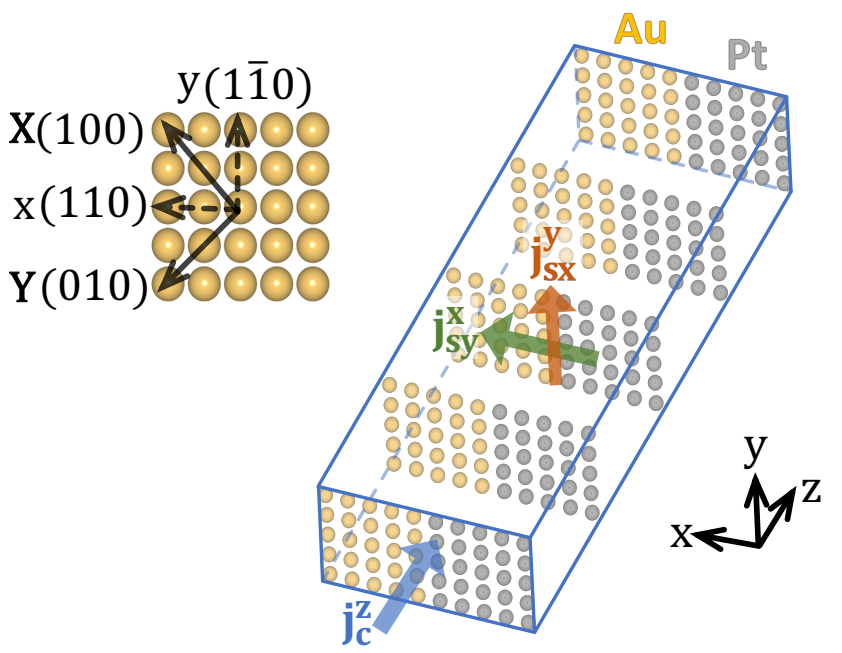

Figure 6.1: Sketch of a $10 \times 5$ lateral supercell used to model transport in a latticematched Pt|Au bilayer. The Pt leads (left, $\mathcal{L}$ and right, $\mathcal{R}$ ) sandwiching this geometry in the $\pm z$-directions are not shown. A charge current $j_{c}^{z}$ (blue arrow) in the [001] $z$ direction generates transverse spin currents like $j_{s y}^{x}$ (green arrow) in the $x$ direction and $j_{s x}^{y}$ (orange arrow) in the $y$ direction. For the fcc lattice used here, the $x$ direction is the crystal [110] direction. Only a few layers in the $z$ direction are explicitly shown for the sake of clarity. For reference, an fcc layer perpendicular to the [001] direction with in-plane crystallographic directions is shown on the left. The conventional cubic axes of an fcc lattice are $X, Y$ and $Z(\equiv z)$

To simulate thermal disorder, atoms are displaced randomly in material $i$ with a Gaussian distribution of displacements characterized by the root mean square displacement $\Delta_{i}$ chosen to reproduce the bulk resistivity $\rho_{i}$ at 300K [150]. For the $\mathrm{FM} \mid \mathrm{NM}$ bilayers, spin disorder is introduced analogously in the FM region where the root mean square rotation of the polar angle with respect to the magnetization direction is chosen to reproduce the bulk magnetization at $300 \mathrm{~K}$ and the azimuthal angle is chosen at random [61]. It has been established that the temperature dependence of the resistance of thin films follows that of the bulk materials $[163,214,215]$ so the use of a bulk $\Delta$ is adequate to describe temperature disorder in our bilayer system.

To study spin injection in a particular direction, in the present case $x$, we use 
$N \times M$ supercells that are sufficiently large in the $x$ direction to recover bulk behaviour in the centres of the individual layers. Since we are limited in the size of lateral supercell that we can handle computationally, we choose $N \gg M$ where $N$ and $M$ are the number of layers in the $x$ and $y$ directions respectively. The asymmetry in supercell dimensions will be taken into account by sampling the BZ asymmetrically so that the sampling density is as close to isotropic as possible. In Fig. 6.2 we plot the $x$ dependence of the intrinsic spin Hall current $\bar{j}_{s y}^{x}(x)$ flowing in the $x$ direction and polarized along $y$ in bulk Pt for a $10 \times 10$ supercell with a BZ sampling equivalent to $160 \times 160$ for a $1 \times 1$ supercell that accurately reproduces the bulk $\Theta_{\mathrm{sH}}^{\mathrm{Pt}}$ of $\sim 4.2 \%$ with a maximum variation of $\sim \pm 0.5 \% . \bar{j}_{s y}^{x}(x)$ is obtained with acceptable variation as a function of $x$ by averaging $j_{s y}^{x}(x, y, z)$ over $y$ and over $z$ omitting the transition region between the ballistic leads and diffusive scattering region.

Unless stated otherwise, all currents will be averaged over $y$ and calculated for a temperature $T=300 \mathrm{~K}$ ("room temperature"); when averaging currents over the scattering region, $z \in \mathcal{S}$, a few layers close to the leads where transient effects are observed are omitted.

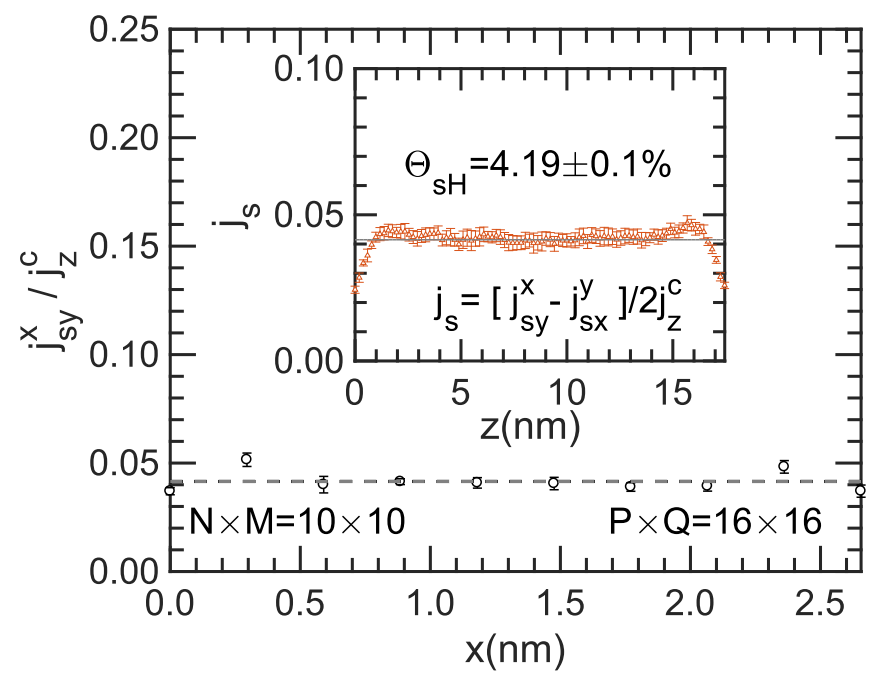

Figure 6.2: A charge current is injected into an $N \times M=10 \times 10$ lateral supercell of $\mathrm{Pt}$ in the $z$ [001] direction leading to transverse spin Hall currents in all directions. We focus on $\bar{j}_{s y}^{x}(x)$, the spin current density in the $x$ direction polarized along $y$ obtained from $j_{s y}^{x}(x, y, z)$ by averaging over the $y$ direction as well as over the $z$ direction (but omitting a transition region at the interface between the leads and the scattering region). The Brillouin zone of the $10 \times 10$ lateral supercell is sampled with $P \times Q=$ $16 \times 16$ k-points. Results are averaged over 10 configurations of random thermal disorder. The inset shows the planar averaged transverse spin currents [20] as a function of $z$ where the interface effects between the ordered leads and disordered scattering region are clearly visible. 


\subsection{Valet-Fert Model}

To interpret the results of the large-scale scattering calculations and make contact with experiment, we begin by adapting a model developed by Valet and Fert [21] to describe spin transport perpendicular to the interfaces of an FM|NM multilayer (current-perpendicular-to-the-plane, $\mathrm{CPP}$ ) to the present case of a $\mathrm{NM} \mid \mathrm{NM}^{\prime}$ multilayer in which a charge current is passed through the multilayer parallel to the interfaces in a so-called current in-plane (CIP) configuration. We will explicitly calculate $j_{c}^{z}(x, y, z)$ and from that obtain $\bar{j}_{c}^{z}(x)$ by averaging over $y$ and $z$. The onedimensional model we will construct will use $\bar{j}_{c}^{z}(x)$ as a source term to drive a spin Hall current in the $x$ direction perpendicular to the $\mathrm{NM} \mid \mathrm{NM}^{\prime}$ interfaces and will include interface effects.

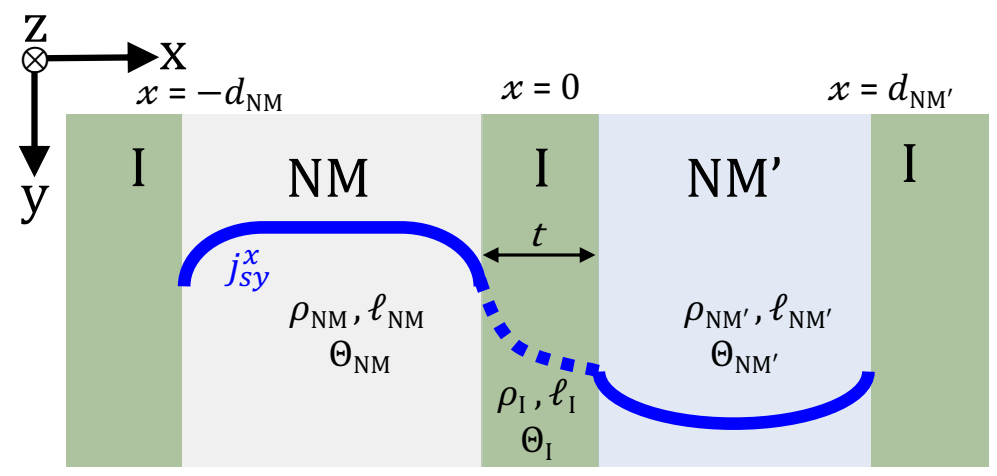

Figure 6.3: Generalized Valet-Fert model for an $\mathrm{NM}|\mathrm{I}| \mathrm{NM}^{\prime}$ multilayer with a charge current $\bar{j}_{c}^{z}(x)$ flowing parallel to the interface planes in the $z$ direction. The charge current drives intrinsic spin Hall currents $j_{s y}^{x}$ (blue curve) in the $x$ and $j_{s x}^{y}$ in the $y$ directions in layer $i$ corresponding to the spin Hall angles $\Theta_{i}$. The material parameters governing the spin currents in layer $i$ are the resistivity $\rho_{i}$ and the spin flip diffusion length $l_{i}$. A spin Hall current is injected from NM into $\mathrm{NM}^{\prime}$ through the interface I.

The $\mathrm{NM} \mid \mathrm{NM}^{\prime}$ bilayer geometry is modelled as an $\mathrm{NM}|\mathrm{I}| \mathrm{NM}^{\prime}$ trilayer with a fictitious "bulklike" interface layer (I) of finite thickness $t$ located between $x=$ $-t / 2$ and $x=t / 2$ with periodic boundary conditions at $x=-d_{\mathrm{NM}}$ and $x=d_{\mathrm{NM}^{\prime}}$ where $d_{\mathrm{NM}}$ and $d_{\mathrm{NM}^{\prime}}$ are the thicknesses of the NM and $\mathrm{NM}^{\prime}$ layers respectively, see Fig. 6.3. A charge current $\bar{j}_{c i}^{z}(x)$ applied parallel to the interface plane in the $z$ direction will excite transverse spin currents $\bar{j}_{c i}^{z}(x) \Theta_{i}$ in each layer $i=$ NM, I and $\mathrm{NM}^{\prime}$ by the spin Hall effect where $\Theta_{i}$ is the spin Hall angle appropriate to layer $i$, $\Theta_{i} \equiv \Theta_{\mathrm{sH}}^{i}$. The total charge current $J_{c}^{z}$ in the $z$ direction is given by

$$
J_{c}^{z}=\int_{-d_{\mathrm{NM}}}^{-t / 2} \bar{j}_{c \mathrm{NM}}^{z}(x) d x+\int_{-t / 2}^{t / 2} \bar{j}_{c \mathrm{I}}^{z}(x) d x+\int_{t / 2}^{d_{\mathrm{NM}}^{\prime}} \bar{j}_{c \mathrm{NM}^{\prime}}^{z}(x) d x
$$


For a total applied charge current $J_{c}^{z}$, the spin transport in the $x$ direction in a bulk layer $i\left(=\mathrm{NM}, \mathrm{I}, \mathrm{NM}^{\prime}\right)$ is characterized by its resistivity $\rho_{i}$, spin flip diffusion length $l_{i}$ and a source term proportional to $\Theta_{i}$. The spin accumulation $\mu_{s}(x)=\mu_{\uparrow}(x)-\mu_{\downarrow}(x)$ and spin-current densities $j_{\uparrow}(x)$ and $j_{\downarrow}(x)$ in the presence of a spin source $\phi_{s}(x)$ in the $x$ direction satisfy the following equations

$$
\begin{aligned}
\frac{\partial^{2} \mu_{s}}{\partial x^{2}} & =\frac{\mu_{s}}{l^{2}}, \\
j_{\uparrow(\downarrow)}(x) & =-\frac{1}{2 e \rho} \frac{\partial \mu_{\uparrow(\downarrow)}(x)}{\partial x}+\phi_{\uparrow(\downarrow)}(x)
\end{aligned}
$$

where $\phi_{\uparrow}(x)-\phi_{\downarrow}(x)=\Theta(x) j_{c}^{z}(x)$ is the intrinsic spin Hall current for any layer $i$. Because there is no charge current in the $x$ direction, we have

$$
\begin{aligned}
& j_{\uparrow}(x)+j_{\downarrow}(x)=0 \\
& =-\frac{1}{2 e \rho}\left[\frac{\partial \mu_{\uparrow}(x)}{\partial x}+\frac{\partial \mu_{\downarrow}(x)}{\partial x}\right]+\phi_{\uparrow}(x)+\phi_{\downarrow}(x) \\
& =-\frac{1}{2 e \rho}\left[2 \frac{\partial \mu_{\uparrow}(x)}{\partial x}-\frac{\partial \mu_{s}(x)}{\partial x}\right]+\phi_{\uparrow}(x)+\phi_{\downarrow}(x) \\
\therefore \quad & \frac{\partial \mu_{\uparrow}(x)}{\partial x}=\frac{1}{2} \frac{\partial \mu_{s}(x)}{\partial x}+e \rho\left[\phi_{\uparrow}(x)+\phi_{\downarrow}(x)\right] .
\end{aligned}
$$

From eq. (6.2) and eq. (6.6) we have

$$
\begin{aligned}
j_{\uparrow}(x) & =-\frac{1}{4 e \rho} \frac{\partial \mu_{s}(x)}{\partial x}+\frac{1}{2}\left[\phi_{\uparrow}(x)-\phi_{\downarrow}(x)\right] \\
j_{\downarrow}(x) & =\frac{1}{4 e \rho} \frac{\partial \mu_{s}(x)}{\partial x}-\frac{1}{2}\left[\phi_{\uparrow}(x)-\phi_{\downarrow}(x)\right] \\
\therefore \quad j_{s}(x) & =j_{\uparrow}(x)-j_{\downarrow}(x)=-\frac{1}{2 e \rho} \frac{\partial \mu_{s}(x)}{\partial x}+\Theta j_{c}^{z}(x)
\end{aligned}
$$

In eq. (6.7c), the $x$ dependence of $\Theta(x)$ is dropped assuming a homogeneous intrinsic spin Hall effect in a given layer. To avoid any ambiguity in the following, we use the notation $j_{s q}^{p}$ for a spin current in the $p$ direction polarized in the $q$ direction and $\mu_{s q}^{p}$ for the corresponding accumulation.

For a spin Hall current in the $x$ direction polarized in the $y$ direction, a general solution of eq. (6.2a) can be written

$$
\mu_{s y}^{x}(x)=A e^{x / l}+B e^{-x / l} .
$$

eq. (6.7c) and eq. (6.8) form the generalized spin transport equations including the spin Hall effect for layer $i$

$$
\begin{aligned}
\mu_{s y i}^{x}(x) & =A_{i} e^{x / l_{i}}+B_{i} e^{-x / l_{i}} \\
j_{s y i}^{x}(x) & =\frac{1}{2 e \rho_{i} l_{i}}\left[B_{i} e^{-x / l_{i}}-A_{i} e^{x / l_{i}}\right]+\Theta_{i} j_{c i}^{z}(x)
\end{aligned}
$$


The spin current $j_{s y}^{x}$ and spin accumulation $\mu_{s y}^{x}$ at the $\mathrm{NM} \mid \mathrm{I}$ and $\mathrm{NM}^{\prime} \mid \mathrm{I}$ interfaces are continuous. We take the limit $t \rightarrow 0$ and introduce an areal resistance $A R_{\mathrm{I}}$ at the interface and an SML parameter $\delta$ to eventually describe a discontinuity in the spin current introduced by the vanishing thickness of the fictitious interface layer I

$$
\lim _{t \rightarrow 0} \rho_{\mathrm{I}} t=A R_{\mathrm{I}} \quad \text { and } \quad \lim _{t \rightarrow 0} t / l_{\mathrm{I}}=\delta .
$$

The fcc superlattice is constructed so as to have inversion symmetry through the centres of the $\mathrm{NM}$ and $\mathrm{NM}^{\prime}$ layers. Making use of this, we finally express the spin current and accumulation in the $\mathrm{NM}$ and $\mathrm{NM}^{\prime}$ layers as

$$
\begin{gathered}
j_{s y i}^{x}(x)=-\frac{A_{i}}{e \rho_{i} l_{i}} \cosh \left(\frac{|x|}{2 l_{i}}-k_{i}\right)+\Theta_{i} j_{c i}^{z}(x) \\
\mu_{s y i}^{x}(x)=2 A_{i} \sinh \left(\frac{|x|}{2 l_{i}}-k_{i}\right)
\end{gathered}
$$

where

$$
\frac{A_{i}}{\rho_{i} l_{i}}=A R_{\mathrm{I}} \frac{\left.\Delta\left(\Theta j_{c}^{z}\right)_{i}\left(e^{2 \delta}+1\right) \delta \rho_{i^{\prime}} l_{i^{\prime}} \sinh k_{i}+\left(e^{2 \delta}-1\right) A R_{\mathrm{I}} \cosh k_{i^{\prime}}\right\}-2 e^{\delta} \Delta\left(\Theta j_{c}^{z}\right)_{i^{\prime}} \delta \rho_{i^{\prime}} l_{i^{\prime}} \sinh k_{i^{\prime}}}{\left(e^{2 \delta}+1\right) \sum_{\alpha}^{i, i^{\prime}} \rho_{\alpha} l_{\alpha} A R_{\mathrm{I}} \delta \cosh k_{\alpha^{\prime}} \sinh k_{\alpha}+\left(e^{2 \delta}-1\right)\left\{\prod_{\alpha}^{i, i^{\prime}} \delta \rho_{\alpha} l_{\alpha} \sinh k_{\alpha}+\prod_{\alpha} A R_{\mathrm{I}} \cosh k_{\alpha}\right\}}
$$

with $\left\{i, i^{\prime}\right\}=\left\{\mathrm{NM}, \mathrm{NM}^{\prime}\right\}$ or $\left\{\mathrm{NM}^{\prime}, \mathrm{NM}\right\}$ and summations or products are over both. eq. (6.9) can be used, after some algebra, to show that the spin currents at the interface $x=0$ satisfy

$$
\begin{aligned}
j_{s y i}^{x}(0)= & \frac{\delta}{A R_{\mathrm{I}} \sinh \delta}\left[\rho_{i} l_{i}\left\{\Theta_{i} j_{c i}^{z}(0)-j_{s y i}^{x}(0)\right\} \tanh k_{i} \cosh \delta+\rho_{i^{\prime}} l_{i^{\prime}}\left\{\Theta_{i^{\prime}} j_{c i^{\prime}}^{z}(0)-j_{s y i^{\prime}}^{x}(0)\right\} \tanh k_{i^{\prime}}\right] \\
& +\Theta_{\mathrm{I}} j_{c \mathrm{Cl}}^{z}\left(x_{\mathrm{I}}\right) \\
j_{s y i^{\prime}}^{x}(0)= & \frac{\delta}{A R_{\mathrm{I}} \sinh \delta}\left[\rho_{i} l_{i}\left\{\Theta_{i} j_{c i}^{z}(0)-j_{s y i}^{x}(0)\right\} \tanh k_{i}+\rho_{i^{\prime}} l_{i^{\prime}}\left\{\Theta_{c i^{\prime}} z_{i^{\prime}}^{z}(0)-j_{s y i^{\prime}}^{x}(0)\right\} \tanh k_{i^{\prime}} \cosh \delta\right] \\
& +\Theta_{\mathrm{I}} j_{c \mathrm{II}}^{z}(0)
\end{aligned}
$$

where $k_{i}=d_{i} / 2 l_{i}, \Delta\left(\Theta j_{c}^{z}\right)_{i}=\Theta_{i} j_{c i}^{z}(0)-\Theta_{\mathrm{I}} j_{c I}^{z}(0)$ and $\Delta\left(\Theta j_{c}^{z}\right)_{i^{\prime}}=\Theta_{i^{\prime}} j_{c i^{\prime}}^{z}(0)-\Theta_{\mathrm{I}} j_{c I}^{z}(0)$. Here, $j_{c i}^{z}(0)$ and $j_{c i^{\prime}}^{z}(0)$ refer to the driving charge current density in layers NM and $\mathrm{NM}^{\prime}$ respectively, close to the interface at $x=0$ and $j_{c i}^{z}(0)$ is the current density through the interface I. How we estimate the values of the charge current densities in Pt|Au will be discussed in Sec. 6.4.1

\subsection{Results}

We begin by presenting the results of scattering calculations of the spin Hall effect for a multilayer comprising two nonmagnetic materials, $\mathrm{Pt}$ and $\mathrm{Au}$ in Sec. 6.4.1 and for a ferromagnetic and nonmagnetic material in Sec. 6.4.2 where FM is Py or Ni. The results for all three interfaces are briefly compared in Sec. 6.4.3. The polarization of the charge current in the $z$ direction is considered in Sec. 6.4.4. All calculations were carried out at $300 \mathrm{~K}$ as described in Sec. 6.2. 


\subsubsection{Pt |Au bilayer}

The results of the first-principles calculations for a Pt|Au (110) multilayer are presented in the following section. After which the parameters needed to describe spin transport with the Valet-Fert model just developed are extracted and discussed.

\section{Spin injection}

The spin Hall angle $\Theta_{\mathrm{sH}}$ is $\sim 4 \%$ in bulk Pt and $\sim 0.25 \%$ in Au [93]. Au has a large spin flip diffusion length $\left(l_{\mathrm{sf}} \sim 50 \mathrm{~nm}\right)$ which results in a very slow decay of an injected spin current making it a much-used spacer layer in experiments. We construct a lattice-matched Pt|Au bilayer as in Fig. 6.1 but now with 60 atomic layers each of Au and Pt in the $x$ direction such that $N=120$ with a total thickness of $33.85 \mathrm{~nm}$ corresponding to the lattice constant of Pt, $a_{\mathrm{Pt}}=0.392 \mathrm{~nm}$. To make the computational effort tractable, we use only $M=3$ layers in the $y$ direction resulting in a supercell size of $120 \times 3$ along with a BZ sampling equivalent to $\sim 133 \times 133$ for a $1 \times 1$ supercell. This BZ sampling density is used throughout for other bilayers as well unless otherwise explicitly stated. The scattering region is constructed by stacking 90 (001) layers $(\sim 17.5 \mathrm{~nm})$ in the $z$ direction.

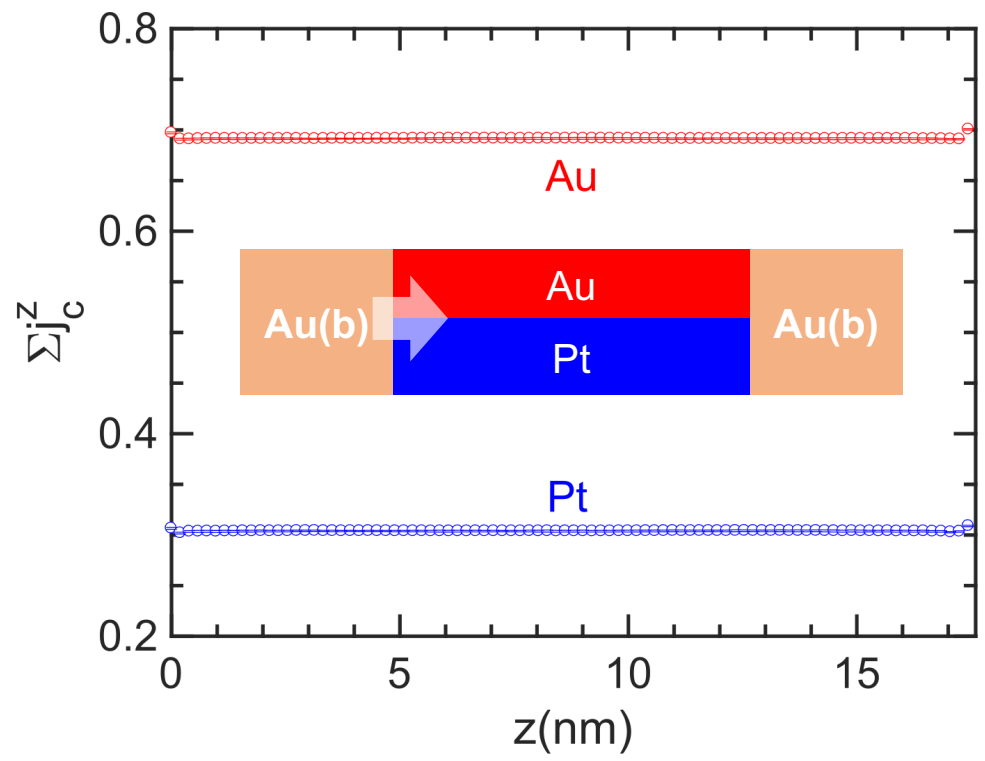

Figure 6.4: A charge current is injected from ballistic Au leads, $\mathrm{Au}(\mathrm{b})$, into the laterally periodic Pt|Au bilayer sketched in the inset. Shown is the total charge current $\Sigma j_{c}^{z}(\mathrm{Pt})$ in Pt and $\Sigma j_{c}^{z}(\mathrm{Au})$ in $\mathrm{Au}$ as a function of the $z$ coordinate. 

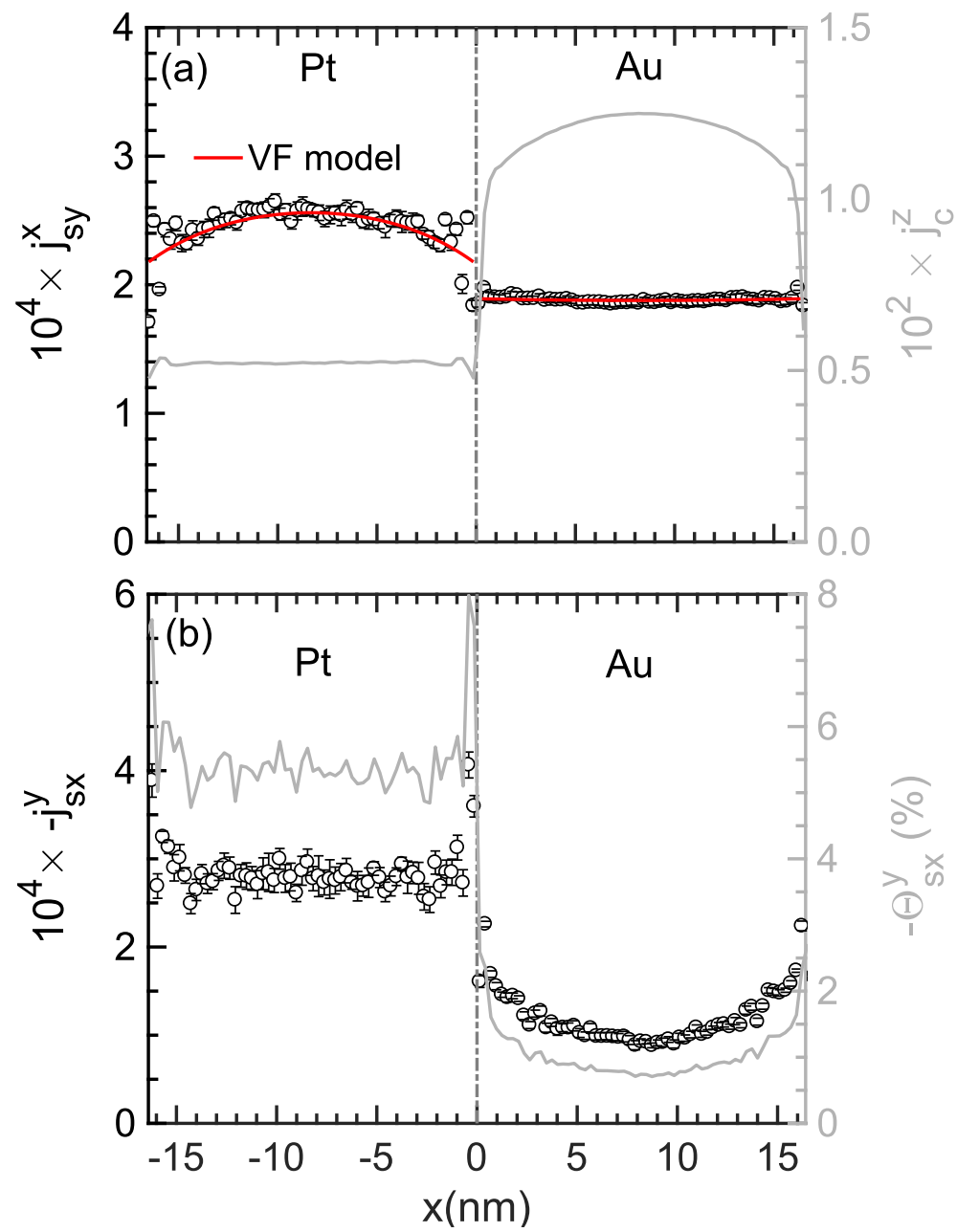

Figure 6.5: Spin Hall currents in a 60Pt|60Au bilayer from scattering calculations. (a) Spin current $\bar{j}_{s y}^{x}(x)$ polarized in the $y$ [110] direction and flowing in the $x$ [11 0 ] direction and the fit using the Valet-Fert (VF) model in red. The distribution of the driving charge current density $\bar{j}_{c}^{z}$ is plotted in grey (right hand axis). (b) Spin current $\bar{j}_{s x}^{y}(x)$ polarized in the $x$ direction and flowing in the $-y$ direction. The local variation of $\Theta_{s x}^{y}(x)=\bar{j}_{s x}^{y}(x) / \bar{j}_{c}^{z}(x)$ is plotted in grey (right hand axis). Error bars represent the average deviation across 10 configurations of thermal disorder.

We calculate $j_{s y}^{x}(x, y, z)$, the spin current in the $x$-direction polarized in the $y$ direction, in response to injecting a charge current $J_{c}^{z}$ in the $z$ direction from $\mathrm{Au}$ leads. With Au leads, the total charge currents in Pt and $\mathrm{Au}, \Sigma j_{c}^{z}(\mathrm{Pt})$ and $\Sigma j_{c}^{z}(\mathrm{Au})$, respectively, have reached their asymptotic values as shown in Fig. 6.4 and are $z$ independent though the distribution within Au has not [205]. The spin current is av- 
eraged over the $y$ direction and in the $z$ direction, omitting a few layers close to the leads to avoid any contact effects. Fig. 6.5(a) shows the averaged spin Hall current $\bar{j}_{s y}^{x}(x)$ flowing in the $x$ direction perpendicular to the interface. The equilibrium distribution of the driving charge current $\bar{j}_{c}^{z}(x)$, also averaged in the $y$ and $z$ directions, is plotted in grey for reference. Corresponding to a total charge current shunting $\Sigma j_{c}^{z}(\mathrm{Au}) / \Sigma j_{c}^{z}(\mathrm{Pt}) \approx 2.24$, we extract an effective Au resistivity $\rho_{\mathrm{Au}}=4.78 \mu \Omega \mathrm{cm}$ using a bulk value of $\rho_{\mathrm{Pt}}=10.7 \mu \Omega \mathrm{cm}$. An appreciable spin injection with little decay inside Au is apparent from the plot of $\bar{j}_{s y}^{x}$. We attribute the oscillatory behaviour of $\bar{j}_{s y}^{x}(x)$ seen in Pt close to the interface to quantum mechanical interference resulting from the finite reflection at the interface [48].

The spin Hall current $\bar{j}_{s x}^{y}(x)$ parallel to the interface is seen in Fig. 6.5(b) to be enhanced close to the interface in both Pt and Au. In Pt it saturates within a few atomic layers to a value corresponding to a spin Hall angle of $\Theta_{s x}^{y} \approx 5.3 \pm 0.4 \%$ that is larger than the value of $4.2 \%$ that we estimate for bulk Pt $[20,93]$. We obtain the same value (not shown) for $\bar{j}_{s y}^{x}(x=0) / \bar{j}_{c}^{z}(x=0)$ implying isotropic, bulk-like behaviour at the centre of the Pt film. The spin current $\bar{j}_{s x}^{y}$ does not saturate inside $\mathrm{Au}$ and is seen to continuously decrease towards the centre of the layer. Since the $\mathrm{Au}$ slab is thinner than $\lambda_{\mathrm{Au}}^{300}$, the mean-free-path at room temperature calculated in the relaxation time approximation [93, 205], we cannot assume isotropy even at the centre of the slab. We use the saturated value $\Theta_{\mathrm{Pt}}=5.3 \%$ and the previously calculated bulk value of $\Theta_{\mathrm{Au}}=0.25 \%$ in our Valet-Fert model. The charge current in Pt is approximately independent of $x$, therefore the source term in eq. (6.11a) is nearly a constant. The charge current in Au has a strong $x$ dependence. However, $\Theta_{\mathrm{Au}}$ is very small compared to $\Theta_{\mathrm{Pt}}$ and therefore the $x$ dependent source term in eq. (6.11a) is small compared to the spin current diffusing into Au from Pt. We neglect the $x$ dependence of the source terms in both layers to simplify the analysis. To do so, we define $j_{c \mathrm{Pt}}^{z}(0)=j_{c \mathrm{Pt}}^{z}, j_{c \mathrm{Au}}^{z}(0)=j_{c \mathrm{Au}}^{z}$ and $j_{c \mathrm{I}}^{z}(0)=\left[j_{c \mathrm{Pt}}^{z}(0)+j_{c \mathrm{Au}}^{z}(0)\right] / 2$ where $j_{c \mathrm{Pt}}^{z}$ and $j_{c \mathrm{Au}}^{z}$ are the charge currents averaged along the $x$ direction inside Pt and Au respectively.

\section{$A R_{\mathrm{I}}, \Theta_{\mathrm{I}}$ and $\delta$}

We follow Gupta et al. and use a CPP configuration in a three step procedure to determine the interface resistance between Pt and $\mathrm{Au}$ [48]. The first step is to calculate the resistance of a length $L_{\mathrm{Pt}}$ of diffusive Pt in series with a length $L_{\mathrm{Au}}$ of diffusive Au sandwiched between ballistic Au leads in the [110] $z$ direction for a variable length of $L_{\mathrm{Pt}}$ and fixed length of $\mathrm{Au}, L_{\mathrm{Au}}=44.21 \mathrm{~nm}$ as shown schematically in Fig. 6.6. The lengths $L_{\mathrm{Pt}}$ and $L_{\mathrm{Au}}$ are longer than the mean free paths in the respective materials and hence the total resistance of the Pt|Au bilayer can be expressed as 


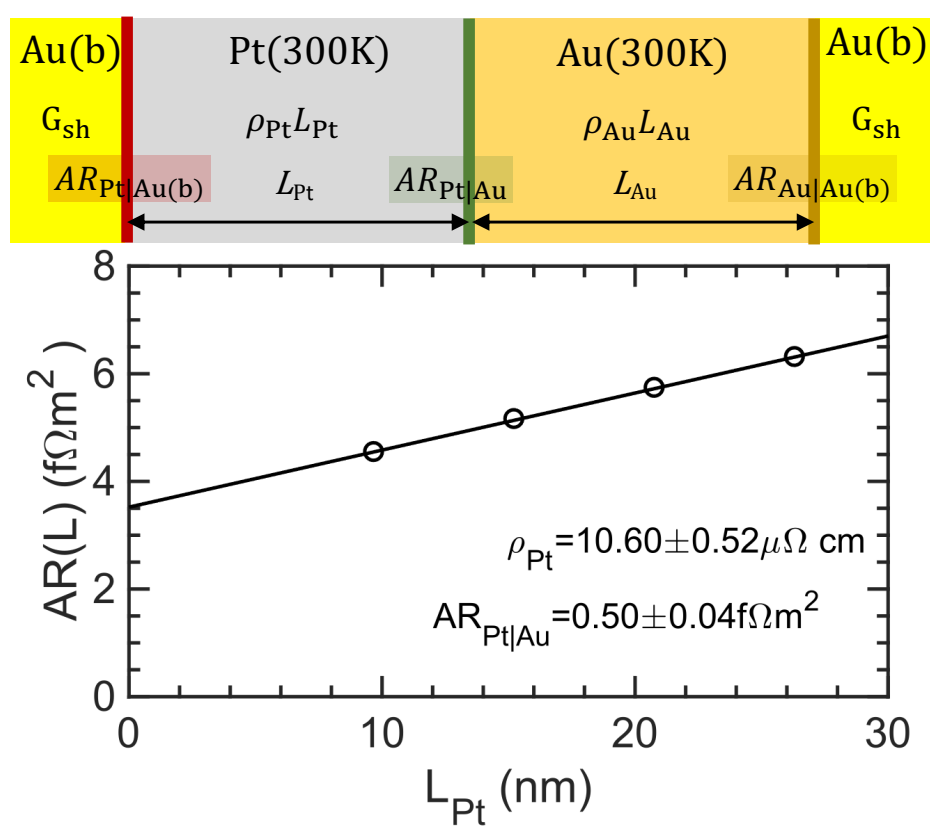

Figure 6.6: Total resistance of a diffusive $\operatorname{Pt}\left(L_{\mathrm{Pt}}\right) \mid \operatorname{Au}\left(L_{\mathrm{Au}}\right)$ (110) junction sandwiched between ballistic Au leads as a function of $L_{\mathrm{Pt}}$ for a fixed length of $L_{\mathrm{Au}}=$ $44.21 \mathrm{~nm}$. The slope corresponds to $\rho_{\mathrm{Pt}}$. The intercept at $L_{\mathrm{Pt}}=0$ contains contributions to the total resistance from ballistic Au leads (yellow), the length $L_{\mathrm{Au}}$ of diffusive $\mathrm{Au}$ (orange), and from the $\mathcal{L} \mid \mathcal{S}$ (red) and $\mathcal{S} \mid \mathcal{R}$ (brown) interfaces that are estimated in separate calculations and subtracted from the intercept to yield $A R_{\mathrm{Pt} \mid \mathrm{Au}}$ (green).

$$
\begin{aligned}
& A R\left(L_{\mathrm{Pt}}, L_{\mathrm{Au}}\right)=\rho_{\mathrm{Pt}} L_{\mathrm{Pt}}+\rho_{\mathrm{Au}} L_{\mathrm{Au}}+A R_{\mathrm{Pt} \mid \mathrm{Au}} \\
& +\left[A R_{\mathrm{Pt} \mid \mathrm{Au}(\mathrm{b})}+1 / G_{\mathrm{Sh}}^{\mathrm{Au}}\right]+\left[A R_{\mathrm{Au} \mid \mathrm{Au}(\mathrm{b})}+1 / G_{\mathrm{Sh}}^{\mathrm{Au}}\right]
\end{aligned}
$$

where $G_{\mathrm{Sh}}^{\mathrm{Au}}$ is the conductance of ballistic gold, the Sharvin conductance [216]. The quantity we are interested in is the areal resistance $A R_{\mathrm{Pt} \mid \mathrm{Au}}$. The other quantities $A R_{\mathrm{Pt} \mid \mathrm{Au}(\mathrm{b})}$ and $A R_{\mathrm{Au} \mid \mathrm{Au}(\mathrm{b})}$ are the areal interface resistances between the left ballistic Au lead and diffusive Pt $(\mathcal{L} \mid \mathcal{S})$ and between the right ballistic Au lead and diffusive $\mathrm{Au}(\mathcal{S} \mid \mathcal{R})$, respectively. These can be determined in separate calculations for variable lengths of diffusive Pt and Au sandwiched between Au leads as shown in Fig. 6.7. For a length $L_{\mathrm{Pt}}$ of diffusive Pt sandwiched between ballistic Au leads, we have $[45,57]$

$$
A R\left(L_{\mathrm{Pt}}\right)=\rho_{\mathrm{Pt}} L_{\mathrm{Pt}}+2\left[A R_{\mathrm{Pt} \mid \mathrm{Au}(\mathrm{b})}+1 / G_{\mathrm{Sh}}^{\mathrm{Au}}\right]
$$



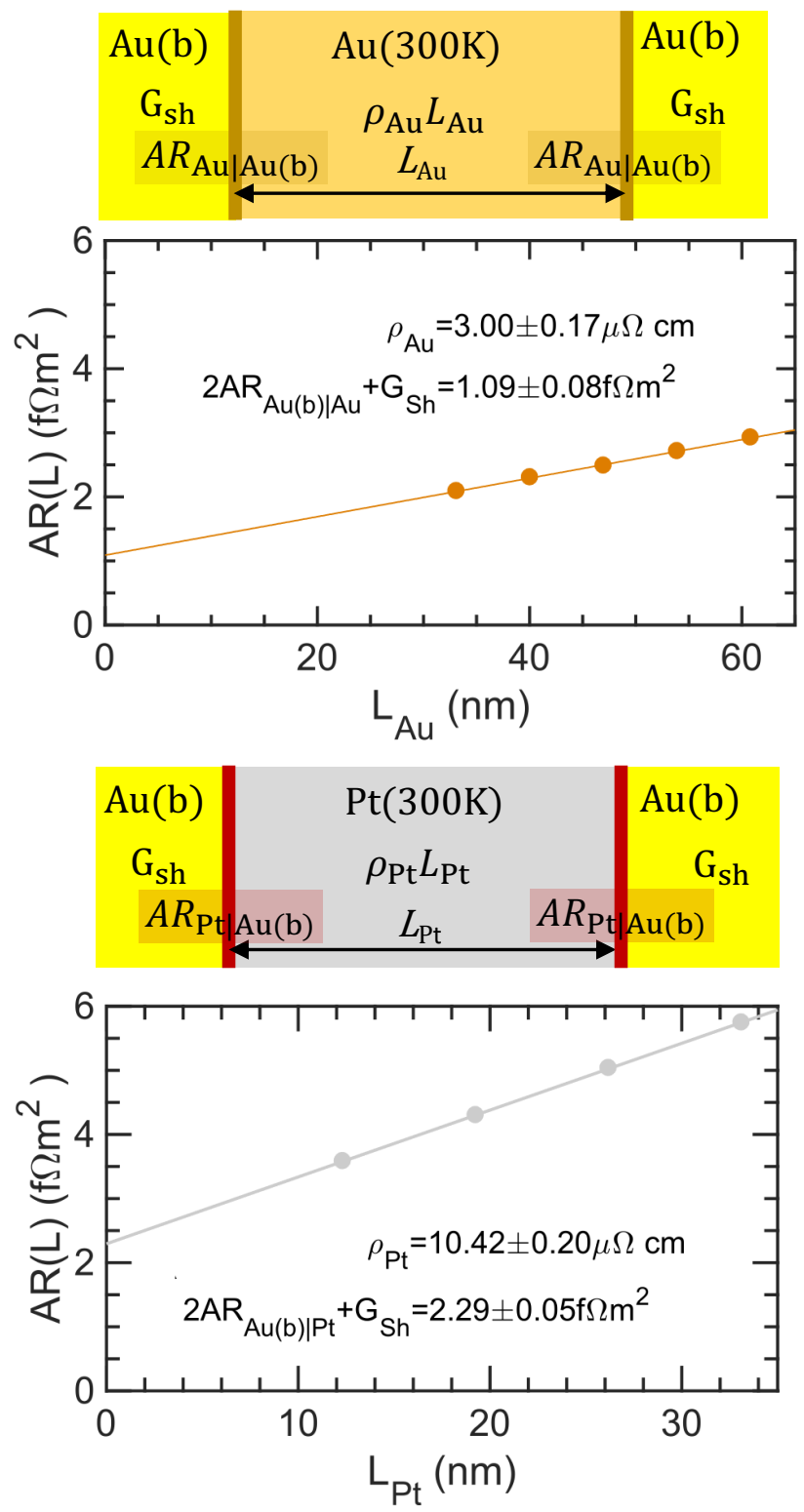

Figure 6.7: Total resistance of diffusive Au (top) and Pt (bottom) sandwiched between ballistic Au leads as a function of the length $L_{\mathrm{Pt}}$ and $L_{\mathrm{Au}}$, respectively. A linear fit of $A R(L)$ to const. $+\rho L$ yields the bulk resistivity as the slope; the intercept is the sum of interface and Sharvin contributions. 
Fitting $A R\left(L_{\mathrm{Pt}}\right)$ gives $\rho_{\mathrm{Pt}}$ as the slope and the second term in the parenthesis as the intercept. Similarly we can determine the final term in eq. (6.14), $A R_{\mathrm{Au} \mid \mathrm{Au}(\mathrm{b})}+$ $1 / G_{\mathrm{Sh}}^{\mathrm{Au}}$ and $\rho_{\mathrm{Au}}{ }^{\dagger}$ using a variable length $L_{\mathrm{Au}}$ of diffusive Au sandwiched between ballistic Au leads. Substituting these values into eq. (6.14) yields $A R_{\mathrm{Pt} \mid \mathrm{Au}}=0.50 \pm$ $0.04 \mathrm{f} \Omega \mathrm{m}^{2}$ approximately equal to the value calculated for a (111) Pt|Au interface [48] using a similar procedure.

The remaining two interface parameters $\delta$ and $\Theta_{\mathrm{I}}$ can be estimated by simultaneously solving equations eq. (6.13) at the interface $x=0$. Calculated $\bar{j}_{s y}^{x}(x)$ exhibit noticeable oscillations at the interface which we attribute to quantum mechanical interference resulting from the finite reflection at the interface. These effects are not described by the semiclassical Valet-Fert framework. To obtain the boundary values $j_{s, \mathrm{Pt}}(0) \equiv \bar{j}_{s y, \mathrm{Pt}}^{x}(0)$ and $j_{s, \mathrm{Au}}(0) \equiv \bar{j}_{s y, \mathrm{Au}}^{x}(0)$ entering eq. (6.13) we need to neglect the quantum mechanical oscillations. To do so, we separately fit eq. (6.11a) to the calculated $\bar{j}_{s y i}^{x}(x)$ in Pt and Au using only the independently determined bulk parameters $l_{i}$ and $\rho_{i}$ where the coefficient $A_{i}$ that captures all the interface parameters is the only unknown parameter of the fit. We then use the best fit to evaluate $j_{s, \mathrm{Pt}}(0)$ and $j_{s, \mathrm{Au}}(0)$. The procedure for solving eq. (6.13) is illustrated in Fig. 6.8. The equations are represented as functions in $\left(\Theta_{\mathrm{I}}, \delta\right)$ space and the solutions are found using standard root searching algorithms. The crossing shows that there is a common solution that satisfies the spin current profiles in $\mathrm{Pt}$ and $\mathrm{Au}$. The solution is unique because it is given by the intersection of functions that are diverging convexly away from the point of intersection that correspond to a value of $\delta=0.57 \pm 0.16$ and $\Theta_{\mathrm{I}}=3.14 \pm 0.2 \%$. The error bars were propagated from the uncertainty in $\bar{j}_{s y, \mathrm{Pt}}^{x}(0)$ and $\bar{j}_{s y, \mathrm{Au}}^{x}(0)$ and other parameters entering eq. (6.13).

The red curves in Fig. 6.5 were then obtained by substituting the values for the interface parameters into eq. (6.11a) and eq. (6.11b) using eq. (6.12) and are found to describe the scattering theory results very well. Within our error bars, the SML parameter $\delta$ extracted here is in agreement with the value determined from the decay of a spin current injected from a polarized lead into a Pt|Au (111) junction (CPP) [48], an entirely different CPP configuration. We used a bulk value $l_{\mathrm{Au}}(300 \mathrm{~K})=50 \mathrm{~nm}$ [93] in our Valet-Fert model whereas the actual value could be lower owing to the enhanced resistivity ${ }^{*}$ but the fit was found to be only weakly sensitive to the value that was used as long as $l_{\mathrm{Au}} \gg d_{\mathrm{Au}}$. The choice of $\Theta_{\mathrm{Au}}$ is also not critical. If we use $\Theta_{\mathrm{Au}}=0.78 \%$ for Au, we find slightly different values of $\delta=0.72$ and $\Theta_{\mathrm{I}}=2.6 \%$ but the same fitting curve.

'The disorder used here was chosen to reproduce the nominal room temperature resistivity of Au with its equilibrium lattice constant. We attribute the enhanced value of resistivity reported here to choosing a smaller lattice constant to match Pt. This was verified by comparing the density of states at the Fermi level which was found to be slightly higher for the smaller lattice.

to estimate a value for $l_{\mathrm{sf}}^{\mathrm{Au}}$ we need to first disentangle the the bulk from the interface contribution to the resistivity, doing so is not trivial. 


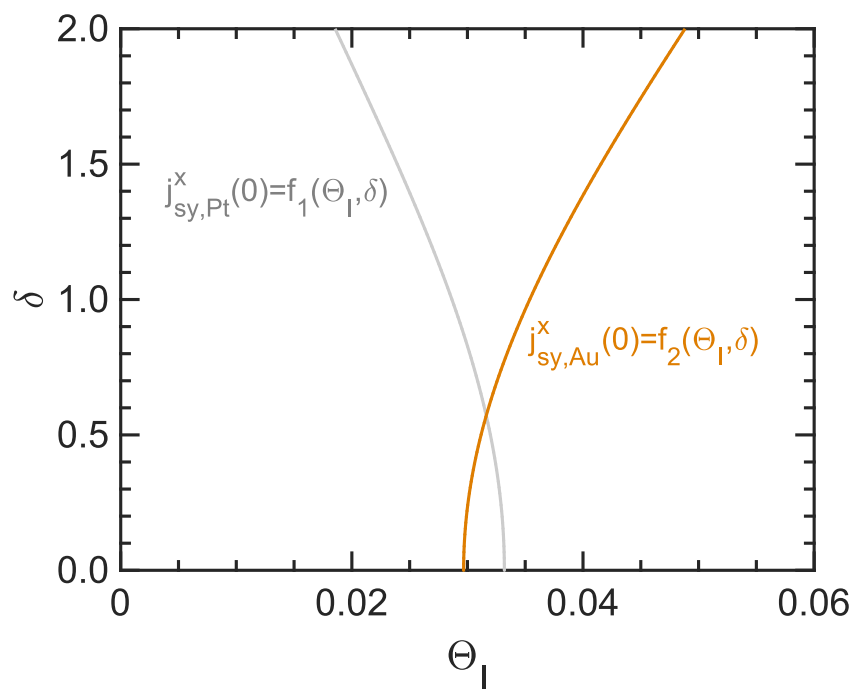

Figure 6.8: Graphical representation of numerical solutions for $\delta$ and $\Theta_{\mathrm{I}}$ obtained by solving eq. (6.13). The common solution is given by the point of intersection of the two curves.

\subsection{2 $\mathrm{FM} \mid \mathrm{Cu}$ bilayers}

Using ab initio calculations, we have shown in chapter 3 that a large spin Hall current can be generated in the bulk of Py and $\mathrm{Ni}$ in agreement with other calculations [38, 137, 157]. Recent experiments using non-local measurements [36, 39] reported a large spin Hall effect in Py. To connect experiments with calculations it is important to investigate how efficiently a pure transverse spin current generated in a FM is transmitted through an FM|NM interface into an adjacent NM layer. A recent attempt to do so [156] in $\mathrm{Co} \mid \mathrm{Cu}$ and $\mathrm{Co} \mid \mathrm{Pt}$ has revealed that a substantial spin current may be generated at the interface and accompany its counterpart generated in the bulk of the FM into the NM layer. To shed more light on this, we look at the spin Hall effect in laterally periodic $\mathrm{FM} \mid \mathrm{Cu}$ bilayers in the following sections. The spin memory loss (SML) at a Py|Cu interface has been reported to be negligible [217] suggesting the suitability of this interface for spin injection while little is known about the SML at a Ni|Cu interface.

In our study of the SHE for bulk Py and $\mathrm{Ni}$ in Chapter 3, we found values of $\Theta_{\mathrm{sH}}^{\mathrm{Py}} \sim 4.8 \%$ and $\Theta_{\mathrm{sH}}^{\mathrm{Ni}} \sim 7.5 \%$ [218] while we calculated a very small value of $\Theta_{\mathrm{sH}}^{\mathrm{Cu}} \sim 0.02 \%$ for $\mathrm{Cu}$ whose $d$ bands are, like those of Au, completely filled. $\mathrm{Cu}$ has a large mean free path ( $~ 39 \mathrm{~nm}$ at $300 \mathrm{~K}$ [49] versus $34 \mathrm{~nm}$ for Au [93]) and, because of its much weaker SOC, a much larger $l_{\text {sf }}$ [219]. Hence, any spin current injected into a $\mathrm{Cu}$ slab of finite thickness of $O(10) \mathrm{nm}$ is expected to pass through with little decay. 
The results to be presented below were obtained from calculations quite analogous to those presented for $\mathrm{Au} \mid \mathrm{Pt}$ with intrinsic thermal disorder chosen to reproduce the experimental room temperature resistivities of bulk $\mathrm{Py}, \mathrm{Ni}$ and $\mathrm{Cu}$. In the FM (= Py or Ni) material, the spin disorder was first chosen to reproduce the experimental magnetization and then the lattice disorder was chosen so that the lattice and spin disorder together reproduced the experimentally observed resistivity at room temperature for the bulk materials [20,57]. Since the lattice mismatch between both Py and $\mathrm{Ni}$ and $\mathrm{Cu}$ is so small, the scattering geometry was constructed by matching the $\mathrm{Cu}$ fcc lattice to that of the equilibrium FM ${ }^{\S}$. A $120 \times 3$ lateral supercell was chosen with 60 layers each of $\mathrm{FM}$ and $\mathrm{Cu}$ in the $x$ direction. In this study, the magnetization direction is set in the $z$ transport direction so that there is no anomalous Hall effect (AHE).

We will not attempt to derive a Valet-Fert type model for the FM|Cu bilayers to describe the transverse spin Hall current analogous to what we presented for the Pt|Au periodic bilayers. Besides the bulk resistivities $\rho_{i}$ and spin-flip diffusion lengths $l_{i}$, the interface resistance $A R_{\mathrm{I}}$ and spin-memory loss $\delta$, the diffusion of a spin current in a periodic $\mathrm{FM} \mid \mathrm{NM}$ bilayer is characterized by the bulk spin polarization $\beta$ in the FM and an interface spin asymmetry parameter $\gamma$ [48]. Furthermore, modelling the transport of transverse spins requires a precession length $l_{p}$, a de-phasing length $l_{\mathrm{dp}}[146,220]$ and their interface counterparts $\delta_{p}$ and $\delta_{\mathrm{dp}}$ in addition to the other interface parameters we defined for Pt|Au making the analysis very cumbersome.

\section{$\mathrm{Py} \mid \mathrm{Cu}$}

We inject a charge current in the $z$ direction into the laterally-periodic latticematched Py|Cu bilayer using ballistic $\mathrm{Cu}(\mathrm{b})$ leads as sketched in Fig. 6.9. There we plot the total charge currents $\Sigma j_{c}^{z}(\mathrm{Py})$ and $\Sigma j_{c}^{z}(\mathrm{Cu})$ inside the Py and $\mathrm{Cu}$ layers, respectively, as a function of $z$. Unlike the case of $\mathrm{Pt} \mid \mathrm{Au}$ where the asymptotic limit was reached immediately, see Fig. 6.4, we observe a weak $z$ dependence of these currents corresponding to the transient shunting of the applied charge current and the asymptotic limit is reached at \pm 10 layers about the centre of the scattering region $(z \sim 8 \mathrm{~nm})$ in the $z$ direction. This could be coming from the fact that there is a significant difference between the transmission of majority and minority spin states at the interfaces between the ferromagnets Py and $\mathrm{Ni}$, and $\mathrm{Cu}[15,16]$, both in the $z$ and in the $x$ directions. To reduce the quantum mechanical fluctuations present in our calculated transverse spin current profile, we neglect any effect of the weak shunting and average the transverse spin current over $z$ omitting five lay-

\footnotetext{
${ }^{\S}$ Because the magnetization of magnetic materials can be very sensitive to even small changes in volume, we prefer to adjust the lattice constant of $\mathrm{Cu}$ to that of FM rather than the other way around. At the Fermi energy, the corresponding change in the electronic structure of a free-electronlike material like $\mathrm{Cu}$ is negligible.
} 
ers close to the leads to avoid transient lead effects.

In Fig. 6.10(a) we plot the spin Hall current $\bar{j}_{s y}^{x}$ in a Py|Cu bilayer along the $x$-direction. We observe a significant injection of spin current from Py into $\mathrm{Cu}$ as we found for Pt|Au. On the Py side of the interface and very close to it, we see a rapid decay of $\bar{j}_{s y}^{x}$. However, the spin current in Py does not have the clear - cosh-like curvature in the bulk that we saw in Pt but is instead reasonably constant with oscillations where the constant value is given by the spin Hall angle, $\Theta_{\mathrm{sH}}^{\mathrm{Py}} \bar{j}_{c}^{z}(x=-7.5 \mathrm{~nm})$ plotted in blue. This implies that the length scale of transverse spin relaxation in Py is miniscule resulting in a vanishingly small diffusive term. We conclude that the significant injection of spin into $\mathrm{Cu}$ is coming effectively from the interface.

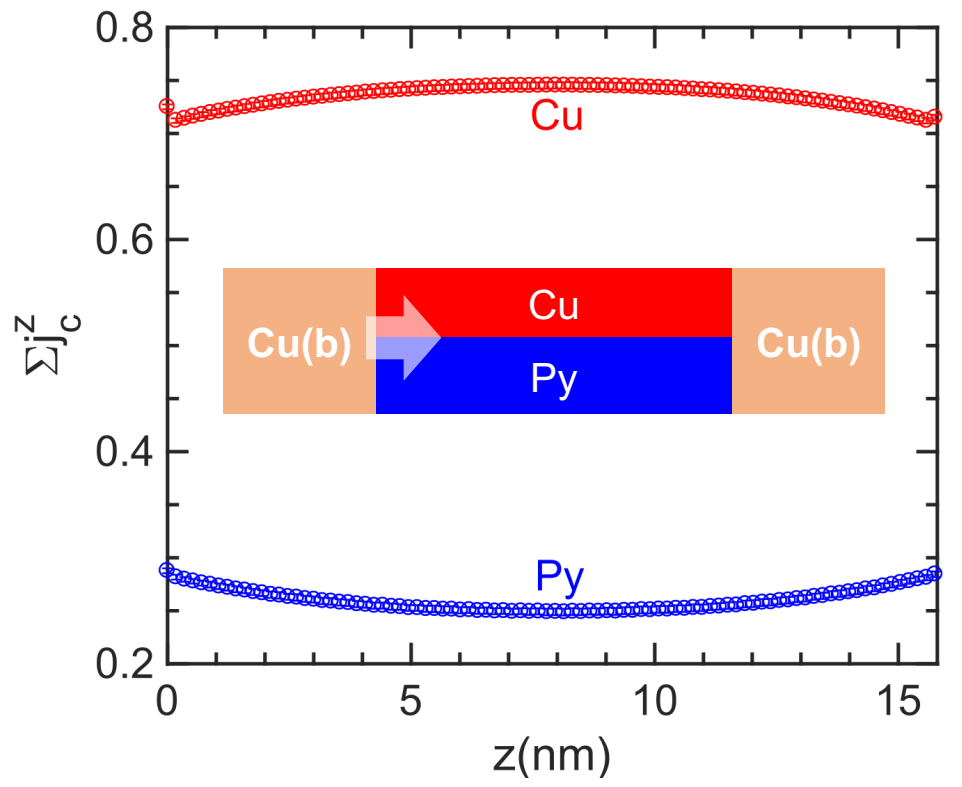

Figure 6.9: A charge current is injected from ballistic $\mathrm{Cu}$ leads, $\mathrm{Cu}(\mathrm{b})$, into the laterally periodic Py|Cu bilayer sketched in the inset. Shown is the total charge current $\Sigma j_{c}^{z}(\mathrm{Py})$ in Py and $\Sigma j_{c}^{z}(\mathrm{Cu})$ in $\mathrm{Cu}$ as a function of the $z$ coordinate where each is obtained by summing over $x, y \in \mathrm{Py}$ and $\mathrm{Cu}$, respectively so $\Sigma j_{c}^{z}(\mathrm{Py})(z)+$ $\sum j_{c}^{z}(\mathrm{Cu})(z)=1$ for all $z$.

The spin current $\bar{j}_{s x}^{y}(x)$ flowing parallel to the interface is plotted in Fig. 6.10(b). It is essentially a constant in Py except for a very small enhancement at the interface. However, the effective local spin Hall angle $\Theta_{s x}^{y}=\bar{j}_{s x}^{y}(x) / \bar{j}_{c}^{z}(x)$ plotted in grey is observed to be slightly suppressed in Py the closer we get to the interface suggesting that the very small enhancement observed in $\bar{j}_{s x}^{y}$ simply follows from an increase in charge current density at the interface plotted in grey in Fig. 6.10(a). Unlike Pt|Au, no significant enhancement of the in-plane spin Hall current is seen 
inside $\mathrm{Cu}$. One possible explanation is that the strong exchange field in Py rapidly quenches any external spins (such as interface generated) not aligned with its magnetization $\mathbf{M}$.

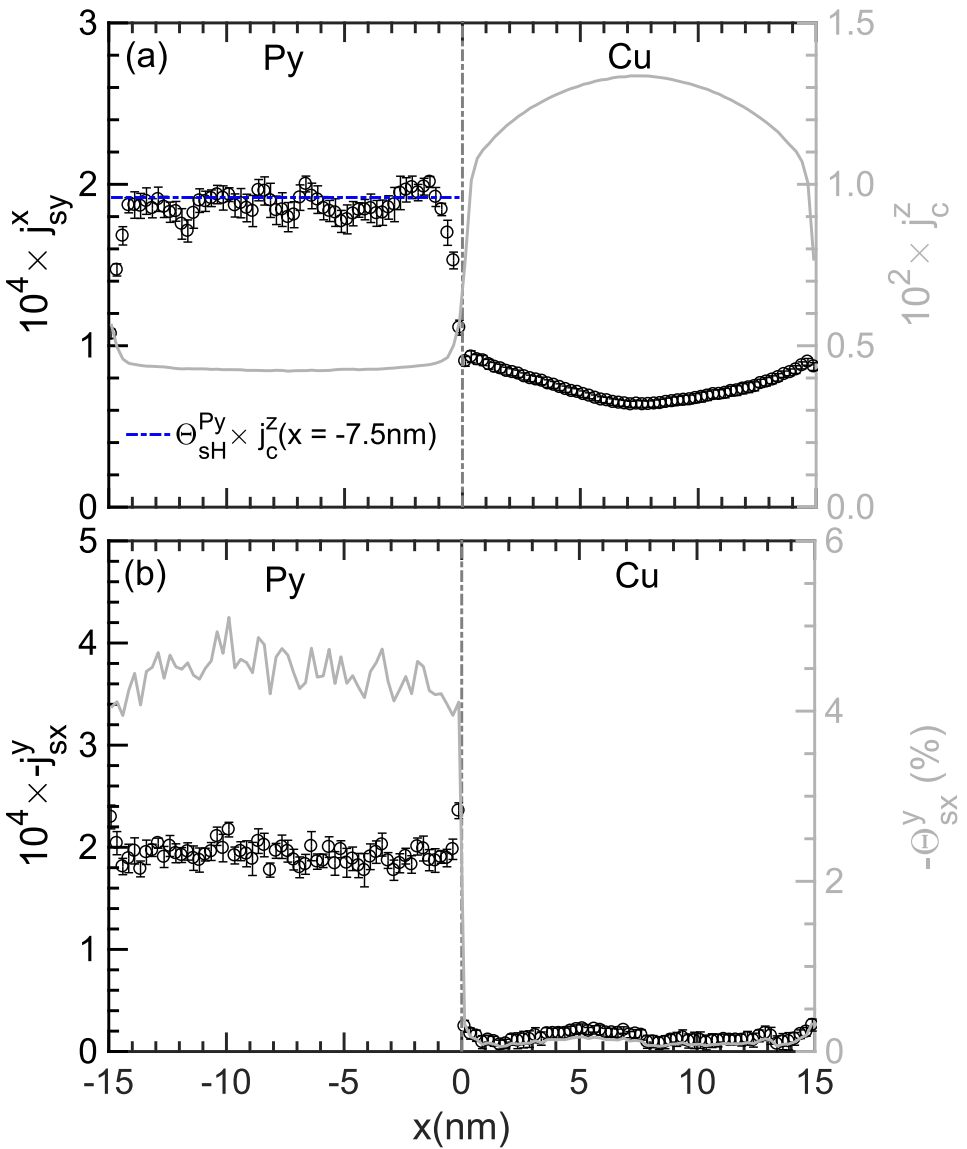

Figure 6.10: Spin Hall effect in a laterally periodic 60Py|60Cu bilayer. (a) Spin current $\bar{j}_{s y}^{x}(x)$ polarized in the $y(110)$ direction and flowing in the $x$ (110) direction. The driving charge current distribution $\bar{j}_{c}^{z}(x)$ is plotted on the right-hand axis. The value $\Theta_{\mathrm{sH}}^{\mathrm{Py}}(=4.5 \%) \times \bar{j}_{c}^{z}(x=-7.5 \mathrm{~nm})$ is plotted in blue in the Py panel for reference. (b) Spin current $\bar{j}_{s x}^{y}(x)$ flowing in the $-y$ (110) direction with polarization in the $x$ (110) direction and the corresponding local variation of the spin Hall angle $\Theta_{s x}^{y}$ on the right axis. Currents are averaged over $y$ and $z$ omitting the five layers closest to the leads. The magnetization of Py is set in the $z$ direction to suppress the anomalous Hall effect. Error bars represent the average deviation across ten configurations of thermal lattice and spin disorder. 


\section{$\mathrm{Ni} \mid \mathrm{Cu}$}

The distribution over the $\mathrm{Ni}$ and $\mathrm{Cu}$ layers of a charge current injected from a ballistic $\mathrm{Cu}$ lead into a $\mathrm{Ni} \mid \mathrm{Cu}$ multilayer is seen in Fig. 6.11 to reflect the weak shunting we saw in $\mathrm{Py} \mid \mathrm{Cu}$. We again neglect this weak $z$ dependence and average the transverse spin Hall currents over $y$ and $z$ to reduce the noise in the calculated data, omitting the five layers closest to the leads.

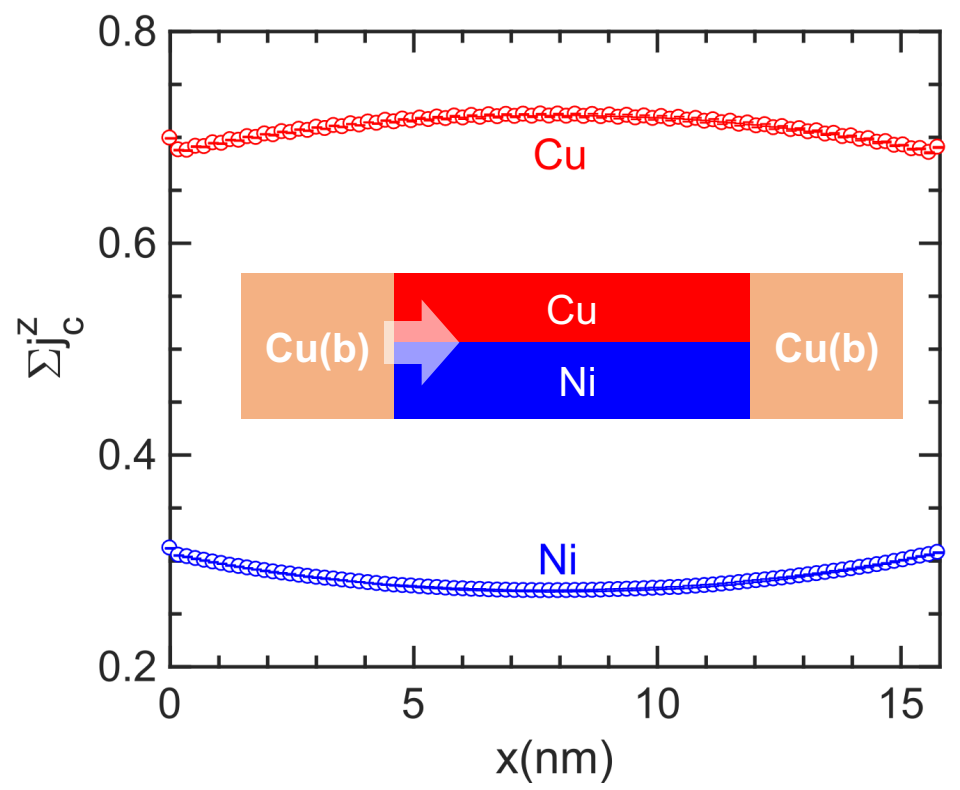

Figure 6.11: A charge current is injected from ballistic $\mathrm{Cu}$ leads, $\mathrm{Cu}(\mathrm{b})$, into the laterally periodic $\mathrm{Ni} \mid \mathrm{Cu}$ bilayer sketched in the inset. Shown is the total charge current $\Sigma j_{c}^{z}(\mathrm{Ni})$ in $\mathrm{Ni}$ and $\Sigma j_{c}^{z}(\mathrm{Cu})$ in $\mathrm{Cu}$ as a function of $z$.

The intrinsically generated spin current $\bar{j}_{s y}^{y}$ flowing perpendicular to the $\mathrm{Ni} \mid \mathrm{Cu}$ interface is shown in Fig. 6.12(a) to be very similar to that in Py|Cu. The spin current is approximately a constant in the bulk corresponding to the spin Hall angle $\Theta_{\mathrm{sH}}^{\mathrm{Ni}}=7.5 \%$. Although the spin Hall angle in Ni is higher than in Py, we do not observe a commensurate increase of spin-injection into $\mathrm{Cu}$; if anything, it is slightly lower. This could be indicative of a larger spin memory loss at the $\mathrm{Ni} \mid \mathrm{Cu}$ interface. The spin current $\bar{j}_{s x}^{y}$ flowing parallel to the interface plotted in Fig. 6.12(c) is observed to be slightly enhanced near the interface in Ni whereas no such effect is seen inside $\mathrm{Cu}$. The effective spin Hall angle $\Theta_{s x}^{y}$ parallels the behaviour found in Py|Cu. 

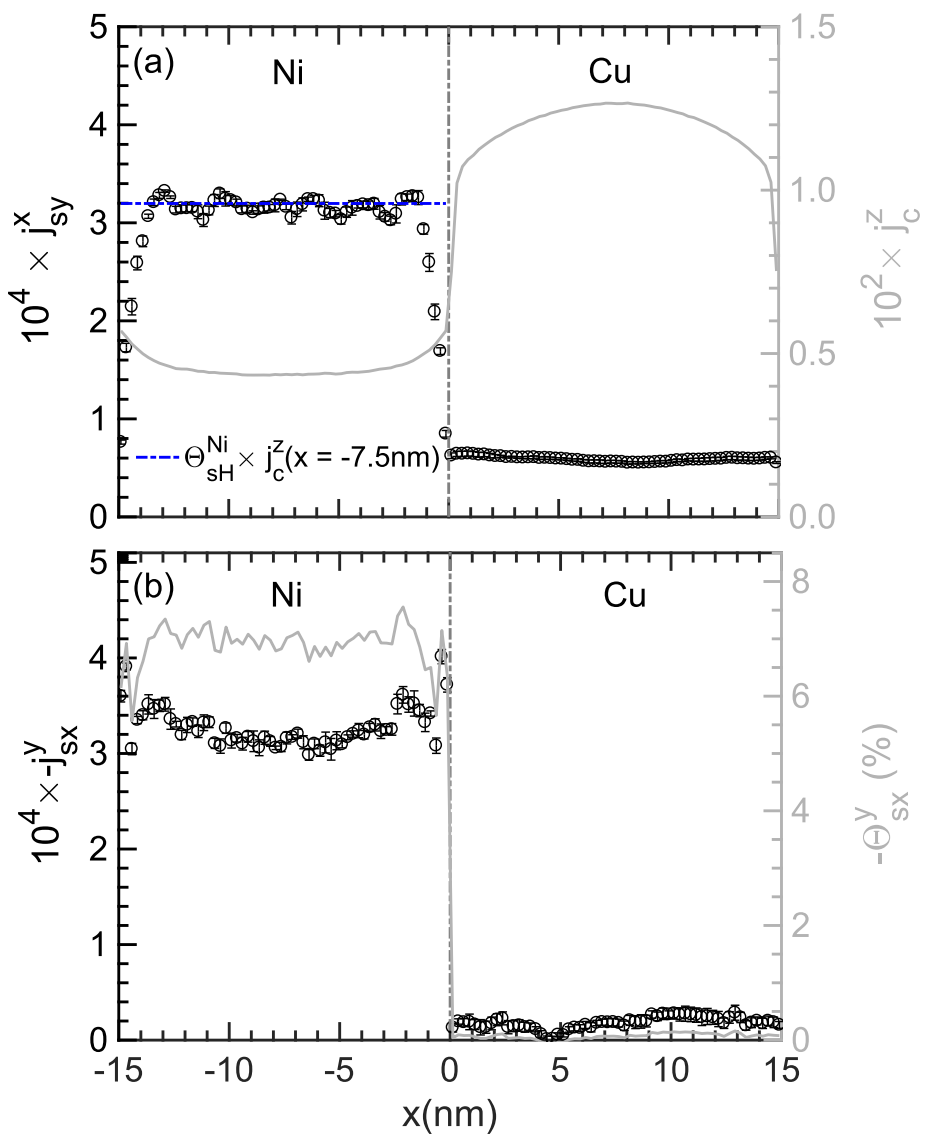

Figure 6.12: Spin Hall effect in a laterally periodic $60 \mathrm{Ni} \mid 60 \mathrm{Cu}$ bilayer. (a) Spin current $\bar{j}_{s y}^{x}(x)$ polarized in the $y(110)$ direction and flowing in the $x$ (110) direction. The driving charge current distribution $\bar{j}_{c}^{z}(x)$ is plotted on the right-hand axis. The value $\Theta_{\mathrm{sH}}^{\mathrm{Ni}}(=7.5 \%) \times \bar{j}_{c}^{\mathrm{z}}(x=-7.5 \mathrm{~nm})$ is plotted in blue in the Ni panel for reference. (b) Spin current $\bar{j}_{s x}^{y}(x)$ flowing in the $-y$ (110) direction with polarization in the $x$ (110) direction and the corresponding local variation of the spin Hall angle $\Theta_{s x}^{y}$ on the right axis. Currents are averaged over $y$ and $z$ omitting the five layers closest to the leads. The magnetization of $\mathrm{Ni}$ is set in the $z$ direction to suppress the anomalous Hall effect. Error bars represent the average deviation across ten configurations of thermal lattice and spin disorder.

\section{Spin swapping}

It was shown in Chapter 3 that in addition to a spin Hall current in a FM, a spin swapping current is allowed by symmetry [40-42]. An out of plane spin current with the symmetry of a spin swapping current has been observed in experiments 

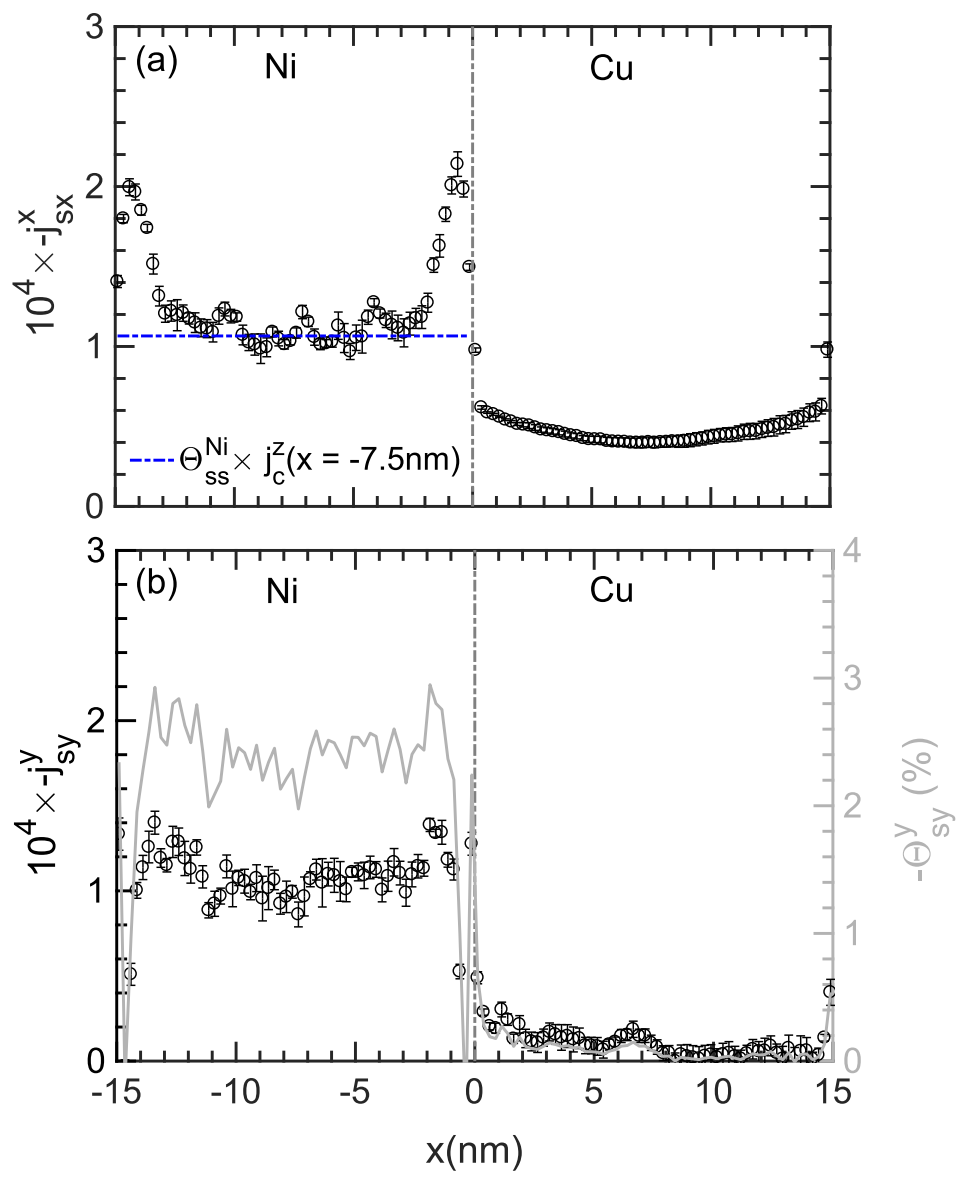

Figure 6.13: Spin swapping in a laterally periodic $60 \mathrm{Ni} \mid 60 \mathrm{Cu}$ bilayer. (a) Spin current $j_{s x}^{x}(x)$ polarized in the $x$ (110) direction and flowing in the $-x$ direction. The reference value $\Theta_{\mathrm{ss}}^{\mathrm{Ni}}(=2.5 \%) \times \bar{j}_{c}^{z}(x=-7.5 \mathrm{~nm})$ is plotted in blue. (b) Spin current flowing in the $-y$ direction with polarization in the $y(\overline{1} 10)$ direction and the corresponding local variation of the spin swapping angle $\Theta_{s y}^{y}$ on the right. Currents are averaged over $y$ and $z$ omitting the five layers closest to the leads. The magnetization of $\mathrm{Ni}$ is set in the $z$ direction to suppress the anomalous Hall effect. Error bars represent the average deviation across 10 configurations of thermal lattice and spin disorder.

$[37,221]$. When the magnetization is aligned parallel to the direction of the driving charge current $j_{c}^{z}$ in our scattering configuration, spin-swapping currents $j_{s x}^{x}$ and $j_{s y}^{y}$ could be excited. A spin-swapping angle $\Theta_{\mathrm{ss}}=j_{s x}^{x} / j_{c}^{\mathrm{z}} \sim-2.5 \%$ calculated for bulk $\mathrm{Ni}$ at $300 \mathrm{~K}$ in chapter 3 should inject a detectable current into $\mathrm{Cu}$. The spin-swapping current $j_{s x}^{x}$ that is found to flow perpendicular to the interface is 
plotted in Fig. 6.13(a). The large peak near the interface decays rapidly into the bulk of Ni over a few layers. The asymptotic value corresponds to the bulk value of $\Theta_{s s}^{\mathrm{Ni}}=2.5 \%$ plotted in blue. A significant SML discontinuity is apparent on the $\mathrm{Cu}$ side of the interface after which the injected current decays only weakly as expected from the long $l_{\mathrm{Cu}}$. The current profile suggests that the injection is bi-directional, from the interface into both $\mathrm{Ni}$ and $\mathrm{Cu}$, with rapid quenching by dephasing inside the FM. Our findings corroborate evidence for large spin-swapping-like currents obtained for $\mathrm{Co} \mid \mathrm{Cu}$ and $\mathrm{Co} \mid \mathrm{Pt}$ interfaces with tight-binding calculations [156]. The spin-swapping current $j_{s y}^{y}$ parallel to the interface is suppressed near the interface, Fig. 6.13(b). Inside $\mathrm{Ni}, j_{s y}^{y}$ saturates to a value roughly corresponding to the bulk value of $\Theta_{\mathrm{ss}} \sim 2.5 \%$ as indicated by the plot of the local spin swapping angle $\Theta_{s y}^{y}$ in grey.

\subsubsection{Comparison}

Table 6.1: Approximate values of spin injection in bilayers considered in this work. For $\mathrm{Ni} \mid \mathrm{Cu}$, the value in brackets corresponds to the injection efficiency of spin swapping current.

\begin{tabular}{cccc}
\hline \hline Par. & $\mathrm{Pt} \mid \mathrm{Au}$ & $\mathrm{Py} \mid \mathrm{Cu}$ & $\mathrm{Ni} \mid \mathrm{Cu}$ \\
\hline$\eta(\%)$ & 57 & 44 & $17(24)$ \\
\hline
\end{tabular}

To quantitatively compare the different bilayers we have considered, we define the following quantity for a general $A \mid B$ bilayer for which $\Theta_{A} \gg \Theta_{B}$.

$$
\eta=\frac{\left(j_{s y B}^{x}-\Theta_{\mathrm{B}} \bar{j}_{c B}^{z}\right)_{x_{\mathrm{I}}}}{\Theta_{A} \bar{j}_{c A}^{z}}
$$

where $\eta$ is the fraction of spin Hall current injected from A into B. The numerator specifies the net spin current density injected at the interface $x_{\mathrm{I}}$ into $\mathrm{B}$ while the denominator gives the average spin Hall current density generated inside A. Estimated values of $\eta$ for the bilayers considered in this study are presented in Table 6.1. Injection of spin Hall current is largest in Pt|Au. It is instructive to note that for the $\mathrm{FM} \mid \mathrm{Cu}$ bilayers considered in this work, the injection efficiency should only be treated as indicative as the injection is predominantly coming from the interface generated current.

\subsubsection{Bulk spin polarization}

A charge current $j_{c}^{z}$ injected into a ferromagnetic material will become spin polarized over a length scale of $l_{\mathrm{sf}}$ saturating to a value corresponding to the conductivity polarization of the bulk ferromagnet, $\beta_{\mathrm{FM}}=\frac{\sigma_{\uparrow}-\sigma_{\downarrow}}{\sigma_{\uparrow}+\sigma_{\downarrow}}$. In Fig. 6.14(a) and (b) we plot 

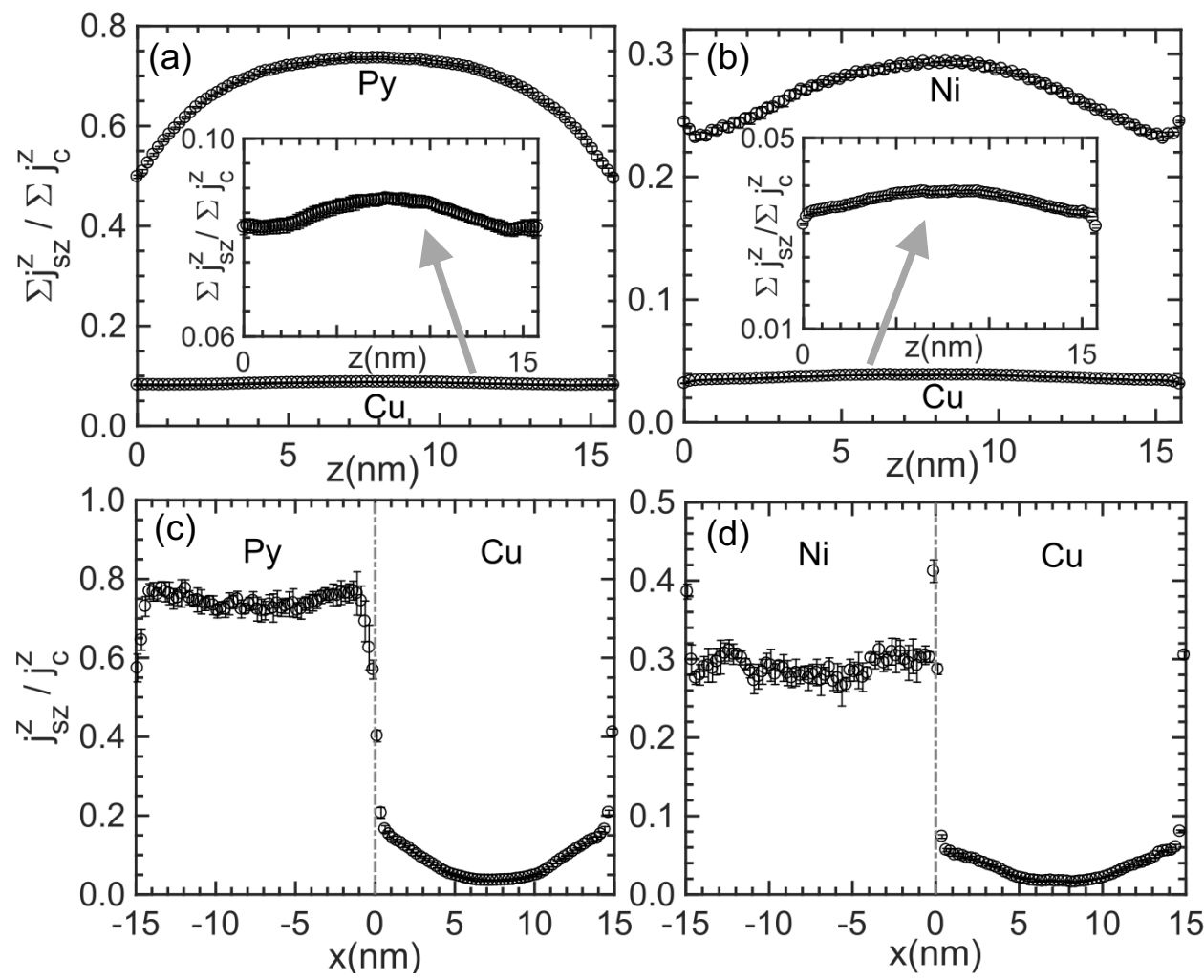

Figure 6.14: Bulk polarization $60 \mathrm{FM} \mid 60 \mathrm{Cu}$ bilayers. A charge current injected in the $z(001)$ direction from $\mathrm{Cu}$ leads becomes spin polarized along the magnetization direction $(z)$ of $\mathrm{Py}(\mathrm{Ni})$ in the scattering region. (a,b) Total spin polarization $\Sigma j_{s z}^{z} / \Sigma j_{c}^{z}$ as a function of position $z$ averaged over $x, y \in \mathrm{FM}$ and $x, y \in \mathrm{Cu}$ where the inset provides a closer look at the spin polarization in $\mathrm{Cu}$. (c,d ) local spin polarization calculated as $j_{s z}^{z}(x) / j_{c}^{z}(x)$ averaged over $y$ and over the ten central layers (about $z=7.8 \mathrm{~nm}$ ) of the scattering region in the $z$ direction. Error bars represent the average deviation across 10 configurations of lattice and spin disorder.

the spin polarization in $\mathrm{Py} \mid \mathrm{Cu}$ and $\mathrm{Ni} \mid \mathrm{Cu}$ in the transport direction i.e along the direction of bulk magnetization of $\mathrm{Py}(\mathrm{Ni})$ averaged in the $x$ and $y$ direction. Inside the FM, the spin polarization reaches a bulk value of $\beta_{\mathrm{Py}} \sim 0.75[20]$ and $\beta_{\mathrm{Ni}} \sim 0.30$ respectively. In both cases, a large polarization is observed in $\mathrm{Cu}$ that appears to parallel the polarization profile in the FM as shown in the insets. In Fig. 6.14(c) and (d) we plot the polarization as a function of $x$ averaged over $y$ and over the ten central layers of the scattering region about $z=7.8 \mathrm{~nm}$ in the $z$ direction. The polarization in the $\mathrm{Cu}$ layers is significant near the interface and tails off to a very small value at the centre of the Cu layer that will be asymptotically zero in the thick $\mathrm{Cu}$ limit, $d \gg l_{\mathrm{Cu}}$. Since our calculations are performed using bulk potentials gen- 
erated separately for $\mathrm{Py}(\mathrm{Ni})$ and $\mathrm{Cu}$, the polarization in $\mathrm{Cu}$ is not the small static polarization of $\mathrm{Cu}$ induced by proximity to $\mathrm{Py}(\mathrm{Ni})$ but arises from leakage (scattering) of the spin polarized current in the adjacent Py(Ni) layers. The preferential scattering of minority spins in a periodic $\mathrm{FM} \mid \mathrm{Cu}$ as shown here is the underlying physics of CIP-GMR.

\subsection{Conclusion}

Using first principles calculations of spatially resolved currents we studied the spin Hall effect in realistic bilayers at room temperature. We demonstrated the significant role played by the interface in lateral spin injection in Pt|Au periodic bilayers. By extending Valet-Fert theory to include interface parameters, we extracted benchmark values for the areal resistance $A R_{\mathrm{I}}$, the spin memory loss $\delta$ and the interface spin Hall angle $\Theta_{\mathrm{I}}$ for an ideal Pt|Au interface. In addition, we found a substantial enhancement of the in-plane spin Hall current close to the Pt|Au interface.

Our calculations revealed previously unknown microscopic details of spin diffusion in realistic FM|NM periodic bilayers resulting from the spin Hall effect at room temperature. We demonstrated unambiguously lateral spin injection by the spin Hall effect from a FM into an adjacent NM material and showed that the injection is predominantly coming from the interface. Based on the large value of its injection efficiency $\eta$, Py|Cu could be a suitable candidate bilayer for SOT based devices. Unlike Pt|Au, the spin current flowing parallel to the interface is not enhanced in $\mathrm{Py}(\mathrm{Ni}) \mid \mathrm{Cu}$ multilayers. In addition to the regular spin Hall current, a detectable spin swapping current is generated at the $\mathrm{Ni} / \mathrm{Cu}$ interface and is injected into both layers. Compared to $\mathrm{Py} \mid \mathrm{Cu}$, a larger SML is apparent at the $\mathrm{Ni} \mid \mathrm{Cu}$ interface. We hope that this work will motivate the extension of a model we developed for lateral spin transport in $\mathrm{NM} \mid \mathrm{NM}$ bilayers to $\mathrm{FM} \mid \mathrm{NM}$ bilayers by incorporating bulk and interface effects on an equal footing. 


\section{A \\ Mott scattering theory}

\section{A.1 Spherical potential}

Consider a local spherical potential $V(r)$ centred at the origin. The time independent Schrödinger equation describing the electron motion is given by

$$
\left[-\frac{\nabla^{2}}{2}+V(r)\right] \psi(r)=E \psi(r)
$$

At a large distance from the origin, the incident wave can be represented as a plane wave. Let us consider the plane wave to be incident in the positive $z$ direction and scattered spherically. Then the total wave function has the form

$$
\psi(r) \underset{r \rightarrow \infty}{\longrightarrow} e^{i k z}+f(\theta) \frac{e^{i k r}}{r}
$$

where $f(\theta)$ is a function of the polar scattering angle $\theta$; there is no $\phi$ dependence because of the symmetry of $V(r)$ and $e^{i k z}$.

\section{A.1.1 Scattering cross-section}

The flux of the scattered electrons through an area $d S$ in the direction specified by $\theta$ and $\phi$ is given by

$$
\text { j.dS }=\frac{1}{2 i}\left[\psi^{*} \nabla \psi-\psi \nabla \psi^{*}\right] . \mathbf{d S}
$$

Then from (A.2) and (A.3) we have,

$$
\mathbf{j . d S}=k|f(\theta)|^{2} d \Omega
$$


The differential cross section is defined as:

$$
\frac{d \sigma}{d \Omega}=\frac{\text { scattered flux } / \text { unit area }}{\text { incident flux } / \text { unit area }}=|f(\theta)|^{2}
$$

\section{A.2 Spin-orbit interaction}

Consider the spin orbit coupling

$$
V_{\mathrm{so}}(r) \mathbf{l} . \mathbf{s}=\frac{1}{2 m^{2} c^{2}} \frac{1}{r} \frac{d V}{d r} \mathbf{l} . \mathbf{s}
$$

where

$$
\mathbf{l . s}=\frac{1}{2}\left(j^{2}-l^{2}-s^{2}\right)
$$

In this case the incident and the scattering wave functions should be represented in terms of the proper spin eigenfunctions say $\chi_{\frac{1}{2}}^{m}$ such that $\chi_{\frac{1}{2}}^{\frac{1}{2}}$ and $\chi_{\frac{1}{2}}^{-\frac{1}{2}}$ correspond to up and down states for instance. Similar to (A.2) one can write the wave function as

$$
\psi(r, \sigma) \underset{r \rightarrow \infty}{\longrightarrow} \chi_{\frac{1}{2}}^{m} e^{i k z}+\sum_{m} \chi_{\frac{1}{2}}^{m} M_{m^{\prime} m}(\theta, \phi) \frac{e^{i k r}}{r}
$$

The scattering amplitude $M(\theta, \phi)$ now contains a spin conserving part $f(\theta)$ as in (A.2) and a spin flipping part $g(\theta) e^{i \phi}$ that allows the spin direction to change in a collision. It is instructive to note that $g(\theta)$ has the form

$$
g(\theta)=\frac{1}{2 i k} \sum_{l=0}^{\infty}\left(e^{2 i \delta_{l}^{l+1 / 2}}-e^{2 i \delta_{l}^{l-1 / 2}}\right) p_{l}^{1}(\cos \theta)
$$

where $\delta_{l}^{l+m}$ is the energy dependent phase shift due to scattering and $p_{l}^{1}(\cos \theta)$ is the associated Legendre function. In the absence of spin-orbit coupling i.e., when $V_{\mathrm{so}}(r)=0, \delta_{l}^{l+1 / 2}=\delta_{l}^{l-1 / 2}$ and thus from (A.9) $g(\theta)=0$ and we recover (A.2).

We can conveniently write $M(\theta, \phi)$ in matrix form as

$$
M(\theta, \phi)=\left(\begin{array}{cc}
f(\theta) & g(\theta) e^{-i \phi} \\
-g(\theta) e^{i \phi} & f(\theta)
\end{array}\right)
$$

Analogous to (A.5)

$$
\frac{d \sigma}{d \Omega}=|f(\theta)|^{2}+|g(\theta)|^{2}
$$

Detailed derivations and rigorous treatment of the scattering amplitudes $f(\theta)$ and $g(\theta)$ can be found in references [27, 144, 145].

Let us define a polarization vector $\mathbf{P}$ with components $P_{x}, P_{y}, P_{z}$ such that

$$
\mathbf{P}=\langle\boldsymbol{\sigma}\rangle \text { with } P_{i}=\frac{\left\langle\chi\left|\sigma_{i}\right| \chi\right\rangle}{\langle\chi \mid \chi\rangle}
$$


where $\chi=\left(\begin{array}{l}a_{1} \\ a_{2}\end{array}\right)$ is a spin state. If we have an ensemble of such spin states with a distribution given by $\lambda^{(n)}$, it is convenient to write down a spin density matrix

$$
\rho=\sum_{n} \lambda^{(n)}\left(\begin{array}{cc}
\left|a_{1}^{(n)}\right|^{2} & a_{1}^{(n)} a_{2}^{(n) *} \\
a_{1}^{(n) *} a_{2}^{(n)} & \left|a_{2}^{(n)}\right|^{2}
\end{array}\right)
$$

Then from (A.12) and (A.13)

$$
\begin{gathered}
\mathbf{P}=\frac{\operatorname{tr}(\rho \boldsymbol{\sigma})}{\operatorname{tr} \rho} \quad \text { and consequently, } \\
\rho=\frac{1}{2}\left(\begin{array}{cc}
1+P_{z} & P_{x}-i P_{y} \\
P_{x}+i P_{y} & 1-P_{z}
\end{array}\right)=\frac{1}{2}(I+\mathbf{P} . \boldsymbol{\sigma}) \operatorname{tr} \rho
\end{gathered}
$$

Now recall that $M(\theta, \phi)$ plays the role of an operator during scattering that transforms an initial state to a scattered (final) state. So if $\rho$ is the initial spin density of the incoming electron beam, then the final spin density $\rho^{\prime}$ is given by

$$
\rho^{\prime}=M \rho M^{\dagger}=\frac{1}{2} M(I+\mathbf{P} . \boldsymbol{\sigma}) M^{\dagger} \operatorname{tr} \rho
$$

so the final polarization will be given by

$$
P^{\prime}=\frac{\operatorname{tr}\left(\rho^{\prime} \sigma\right)}{\operatorname{tr} \rho^{\prime}}
$$

The evaluation of $P^{\prime}$ thus involves

$$
\begin{array}{r}
\operatorname{tr}\left(\rho^{\prime} \sigma\right)=\frac{1}{2} \operatorname{tr}\left(\begin{array}{cc}
f(\theta) & g(\theta) e^{-i \phi} \\
-g(\theta) e^{i \phi} & f(\theta)
\end{array}\right)\left(\begin{array}{cc}
1+P_{z} & P_{x}-i P_{y} \\
P_{x}+i P_{y} & 1-P_{z}
\end{array}\right) \\
\left(\begin{array}{cc}
f^{*}(\theta) & -g^{*}(\theta) e^{-i \phi} \\
g^{*}(\theta) e^{i \phi} & f^{*}(\theta)
\end{array}\right)\left(\begin{array}{cc}
\sigma_{z} & \sigma_{x}-i \sigma_{y} \\
\sigma_{x}+i \sigma_{y} & -\sigma_{z}
\end{array}\right) \\
\operatorname{tr} \rho^{\prime}=\frac{1}{2} \operatorname{tr}\left(\begin{array}{cc}
f(\theta) & g(\theta) e^{-i \phi} \\
-g(\theta) e^{i \phi} & f(\theta)
\end{array}\right)\left(\begin{array}{cc}
1+P_{z} & P_{x}-i P_{y} \\
P_{x}+i P_{y} & 1-P_{z}
\end{array}\right) \\
\left(\begin{array}{cc}
f^{*}(\theta) & -g^{*}(\theta) e^{-i \phi} \\
g^{*}(\theta) e^{i \phi} & f^{*}(\theta)
\end{array}\right)
\end{array}
$$

Evaluating (A.17) and (A.18) and substituting in (A.16), $P^{\prime}$ reduces to the form

$$
\mathbf{P}^{\prime}=\frac{[\mathbf{P} . \hat{\mathbf{n}}+S(\theta)] \hat{\mathbf{n}}+T(\theta) \hat{\mathbf{n}} \times[\mathbf{P} \times \hat{\mathbf{n}}]+U(\theta)[\hat{\mathbf{n}} \times \mathbf{P}]}{1+\mathbf{P} . \hat{\mathbf{n}} S(\theta)}
$$


where

$$
\begin{aligned}
& S(\theta)=i \frac{f g^{*}-f^{*} g}{|f|^{2}+|g|^{2}}, \\
& T(\theta)=\frac{|f|^{2}-|g|^{2}}{|f|^{2}+|g|^{2}}, \\
& U(\theta)=\frac{f g^{*}+f^{*} g}{|f|^{2}+|g|^{2}}
\end{aligned}
$$

\section{A.3 Spin Hall effect}

Now let us examine if the spin Hall effect can be explained as a natural consequence of the scattering process from a spin-orbit potential. Consider the Pauli spin matrices $\sigma_{x}, \sigma_{y}, \sigma_{z}$ and the identity matrix $I$

$$
\sigma_{x}=\left(\begin{array}{ll}
0 & 1 \\
1 & 0
\end{array}\right), \sigma_{y}=\left(\begin{array}{cc}
0 & -i \\
i & 0
\end{array}\right), \sigma_{z}=\left(\begin{array}{cc}
1 & 0 \\
0 & -1
\end{array}\right), I=\left(\begin{array}{ll}
1 & 0 \\
0 & 1
\end{array}\right)
$$

then $M(\theta, \phi)$ can be expressed in terms of $\sigma_{x}, \sigma_{y}, \sigma_{z}$ and $I$ as

$$
M(\theta, \phi)=f(\theta) I-i \sigma_{x} g(\theta) \sin \phi+i \sigma_{y} g(\theta) \cos \phi
$$

Consider the case of an unpolarised wave of electrons (charge current) along the $z$ axis being scattered along the $x$ axis by the spin-orbit interaction. $\therefore \phi=0$ we have from (A.21)

$$
M(\theta, \phi)=f(\theta)+i g(\theta) \sigma_{y}
$$

thus the beam scattered along $x$ is polarized in $y$. This result can be written for a general case

$$
M(\theta, \phi)=f(\theta) I+i g(\theta) \sigma . \hat{\mathbf{n}}
$$

with $\hat{\mathbf{n}}=\frac{\mathbf{k}_{i} \times \mathbf{k}_{f}}{\left|\mathbf{k}_{i} \times \mathbf{k}_{f}\right|}$ where $\mathbf{k}_{i}$ and $\mathbf{k}_{f}$ are the initial and final directions of the electron respectively. or more generally using (A.19), setting $P=0$ for an unpolarized incident beam we get

$$
\mathbf{P}^{\prime}=S(\theta) \hat{\mathbf{n}}
$$

This is essentially the underlying mechanism of transverse spin current generation in a solid by spin-orbit coupling. Now for a magnetic material we can imagine a part of the incident charge current getting polarized in the direction of bulk magnetization. Let us consider the case of magnetization along $\mathbf{k}_{i}$ i.e., along the incident direction then from (A.19)

$$
\mathbf{P}^{\prime}=S(\theta) \hat{\mathbf{n}}+T(\theta) P \hat{\mathbf{k}}_{i}+U(\theta) P \hat{\mathbf{k}}_{f}
$$

The component $U(\theta) P \hat{\mathbf{k}}_{f}$ represents a transverse current component in addition to the "usual" spin Hall component in magnetic materials. It is self evident that 
this component will change signs when the magnetization is reversed due to the cross product. Such a transverse current generated due to a polarized current is also referred to as a spin swapping current and was derived using a semi-classical approach [40].

The component $T(\theta) P \hat{\mathbf{k}}_{i}$ is the diffusive Elliot-Yafet component.

\section{A.4 Skew and side jump scattering}

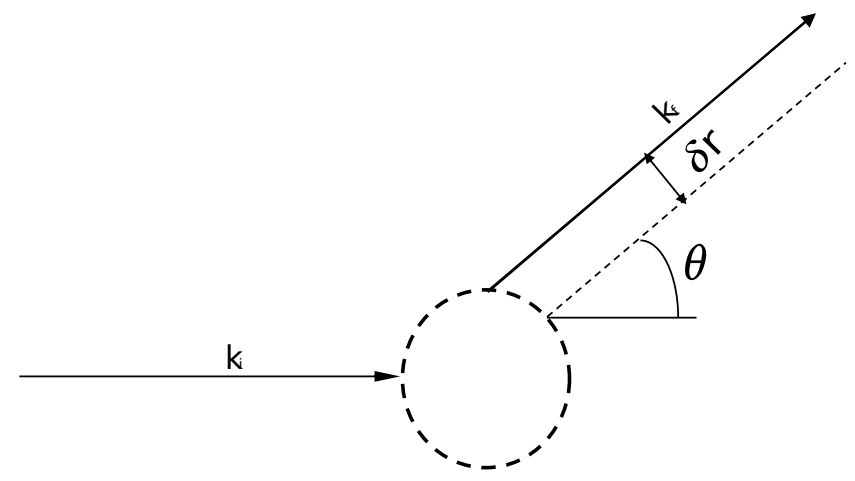

Figure A.1: Scattering of an electron by a spherical impurity centre. The skew scattering leads to a finite scattering amplitude directed at an angle $\theta$ with respect to the initial direction while the side-jump contribution shifts the scattered electron by $\delta r$.

Figure A.1 shows the two important scattering mechanisms in play at $\mathrm{T}=0 \mathrm{~K}$. Skew scattering of itinerant electrons in solids due to the asymmetric scattering by a spin-orbit potential was discussed in detail by Smit [222]. We will present a simple picture of skew scattering in the spirit of multiple scattering. Consider scattering events $k \rightarrow k^{\prime}$ and $k^{\prime} \rightarrow k$ with scattering rates $P_{k k^{\prime}}$ and $P_{k^{\prime} k}$. In order to have a finite current we have

$$
\left|P_{k k^{\prime}}-P_{k^{\prime} k}\right|>0
$$

By applying Fermi's golden rule we have

$$
P_{k k^{\prime}}-P_{k^{\prime} k}=\frac{\pi}{\hbar} N\left(\left|T_{k k^{\prime}}\right|^{2}-\left|T_{k k^{\prime}}\right|^{2}\right) \delta\left(E_{k}-E_{k^{\prime}}\right)
$$

where $N$ is the number of scattering events and $T_{k k^{\prime}}$ is the transition probability of the scattering event $k \rightarrow k^{\prime}$. Note that we dropped a factor 2 in Fermi's golden rule since we are interested in the sum of all pairs of events like $k \rightarrow k^{\prime}$ and $k^{\prime} \rightarrow k$. Applying the famous optical theorem and by making use of the definition of $T_{k k^{\prime}}$ 
[223] (A.27) becomes

$$
P_{k k^{\prime}}-P_{k^{\prime} k}=\frac{8 \pi^{2} \hbar^{3}}{m_{e}^{2} V^{2}} \operatorname{Im}\left(\mathrm{M}_{\mathrm{kk}^{\prime}}^{2}-\mathrm{M}_{\mathrm{k}^{\prime} \mathrm{k}}^{2}\right)
$$

where $m_{e}$ and $V$ are the electron mass and volume in which scattering occurs respectively. Note that we recast the scattering amplitude $M(\theta, \phi)$ as $M_{k k^{\prime}}$. Equation (A.28) can be treated semi-classically using the Boltzman equation to obtain the skew scattering elements of the conductivity tensor [224]. It is also instructive to note that $\left|T_{k k^{\prime}}^{\uparrow \uparrow}\right|^{2}-\left|T_{k^{\prime} k}^{\uparrow \uparrow}\right|^{2}$ is an antisymmetric matrix which gives rise to different signs of spin current generated along two directions orthogonal to the incident direction as required by symmetry.

We will now attack the case of side jump scattering. Consider the following Hamiltonian describing the motion of an electron in a solid

$$
H=\frac{p^{2}}{2 m}+U(\mathbf{r})+\Gamma[\nabla U(\mathbf{r}) \times \mathbf{s}] . \mathbf{p}
$$

the first two terms are the usual kinetic and potential energy terms while the last term gives the spin-orbit coupling energy with an appropriate scaling $\Gamma$. From (A.29) we have

$$
\begin{gathered}
\dot{\mathbf{r}}=\frac{\partial H}{\partial \mathbf{p}}=\frac{\mathbf{p}}{m}+\Gamma(\nabla U \times \mathbf{s}) \\
\dot{\mathbf{p}}=-\frac{\partial H}{\partial \mathbf{r}}=-\nabla U-\mathbf{p} . \nabla \Gamma(\nabla U \times \mathbf{s})
\end{gathered}
$$

where we have used $U \rightarrow U(r)$. The second term in (A.31) is second order in the spin-orbit interaction and can be conveniently neglected in the limit of weak spinorbit coupling. Hence from (A.30) and (A.31) we have

$$
\dot{\mathbf{r}}=\frac{\mathbf{p}}{m}+\Gamma(\mathbf{s} \times \dot{\mathbf{p}})
$$

we can now write

$$
\dot{\mathbf{r}}=\dot{\mathbf{r}}_{0}+\dot{\delta} r
$$

where $\dot{\mathbf{r}}_{0}$ is the "usual velocity" given by the first term in (A.32) and $\dot{\delta} r$ can be thought of as an additional velocity due to the spin-orbit term which must be caused by the side jump during scattering if we neglect other effects. Thus we have

$$
\delta v_{s j}=\Gamma(s \times \dot{p})
$$

Instead of integrating this equation and evaluating the side jump $\delta r$, we proceed with finding the expectation value of the side jump velocity in order to make contact with the scattering amplitude $M(\theta, \phi)$

$$
<\delta v_{s j}>=\left\langle\psi_{k}|\Gamma(s \times \dot{p})| \psi_{k}\right\rangle
$$


Using the generalised Ehrenfest theorem [225] the right hand side of (A.35) transforms to

$$
\left\langle\psi_{k}|\Gamma(s \times \dot{p})| \psi_{k}\right\rangle=\sum_{f} \Gamma\left(s \times\left(p_{f}-p_{i}\right)\right) w_{f i}
$$

where $p_{i}$ and $p_{f}$ are the incident and scattered momentum respectively and $w_{f i}$ is the rate of change of scattering probability. Recalling the definition of scattering cross section $\sigma$, one may write

$$
w_{f i}=\sigma v
$$

where $v$ is the electron velocity. Substituting $p=\hbar k$ and using equations (A.36) and (A.37), equation (A.35) reduces to

$$
<\delta v_{s j}>=\sum_{\theta} \Gamma^{\prime}(s \times k)(1-\cos (\theta)) \sigma v
$$

where $\Gamma^{\prime}=\hbar \Gamma$ and $\theta$ is the angle between initial and final $k$ vectors in a scattering event and $\sigma$ is given by $|M(\theta, \phi)|$ as in (A.11). We found that a similar result for side jump was arrived through an alternate route in [226]. It is worthwhile noting that there can be an additional side jump comparable to $\delta r_{s j}$ as a result of the dipole generated immediately after scattering, which was rigorously treated in [227]. 


\section{Bibliography}

[1] A. H. Compton, "The magnetic electron", Journal of the Franklin Institute 192, 145155 (1921).

[2] R. Kronig, "The turning point", in Theoretical Physics in the twenteeth century: a memorial volume to Wolfgang Pauli, edited by M. Fierz and V. F. Weisskopf (Interscience Publishers INC, New York) (1960).

[3] G. E. Uhlenbeck and S. Goudsmit, "Spinning electrons and the structure of spectra", Nature (London) 117, 164 (1926).

[4] C. Chappert, A. Fert, and F. N. Van Dau, "The emergence of spin electronics in data storage", Nat. Mater. 6, 813-823 (2007).

[5] M. N. Baibich, J. M. Broto, A. Fert, F. Nguyen Van Dau, F. Petroff, P. Etienne, G. Creuzet, A. Friederich, and J. Chazelas, "Giant Magnetoresistance of (001)Fe/(001)Cr Magnetic Superlattices", Phys. Rev. Lett. 61, 2472-2475 (1988).

[6] G. Binasch, P. Grünberg, F. Saurenbach, and W. Zinn, "Enhanced magnetoresistance in layered magnetic structures with antiferromaganetic interlayer exchange", Phys. Rev. B 39, 4828-4830 (1989).

[7] T. Miyazaki and N. Tezuka, "Giant magnetic tunneling effect in $\mathrm{Fe} / \mathrm{Al}_{2} \mathrm{O}_{3} / \mathrm{Fe}$ junction”, J. Magn. Magn. Mater. 139, L231-L234 (1995).

[8] J. S. Moodera, L. R. Kinder, T. M. Wong, and R. Meservey, "Large Magnetoresistance at Room Temperature in Ferromagnetic Thin Film Tunnel Junctions", Phys. Rev. Lett. 74, 3273-3276 (1995).

[9] S. Ikeda, J. Hayakawa, Y. Ashizawa, Y. M. Lee, K. Miura, H. Hasegawa, M. Tsunoda, F. Matsukura, and H. Ohno, "Tunnel magnetoresistance of 604pseudo-spin-valves annealed at high temperature", Appl. Phys. Lett. 93, 082508 (2008).

[10] G. A. Prinz, "Magnetoelectronics”, Science 282, 1660-1663 (1998).

[11] A. S. Núñez and A. H. MacDonald, "Theory of spin transfer phenomena in magnetic metals and semiconductors", Solid State Commun. 139, 31-34 (2006).

[12] J. E. Hirsch, "Spin Hall Effect”, Phys. Rev. Lett. 83, 1834 (1999).

[13] N. F. Mott, "The electrical conductivity of transition metals", Proc. R. Soc. London, Ser. A 153, 699-717 (1936). 
[14] K. M. Schep, P. J. Kelly, and G. E. W. Bauer, "Giant Magnetoresistance without Defect Scattering", Phys. Rev. Lett. 74, 586-589 (1995).

[15] K. M. Schep, P. J. Kelly, and G. E. W. Bauer, "Ballistic transport and electronic structure”, Phys. Rev. B 57, 8907-8926 (1998).

[16] K. Xia, M. Zwierzycki, M. Talanana, P. J. Kelly, and G. E. W. Bauer, "First-principles scattering matrices for spin-transport", Phys. Rev. B 73, 064420 (2006).

[17] F. Herman and S. Skillman, Atomic Structure Calculations (Prentice Hall, Englewood Cliffs, New Jersey) (1963).

[18] J. Bass and W. P. Pratt Jr., "Spin-diffusion lengths in metals and alloys, and spinflipping at metal/metal interfaces: an experimentalist's critical review", J. Phys.: Condens. Matter 19, 183201 (2007).

[19] J.-C. Rojas-Sánchez, N. Reyren, P. Laczkowski, W. Savero, J.-P. Attané, C. Deranlot, M. Jamet, J.-M. George, L. Vila, and H. Jaffrès, "Spin Pumping and Inverse Spin Hall Effect in Platinum: The Essential Role of Spin-Memory Loss at Metallic Interfaces", Phys. Rev. Lett. 112, 106602 (2014).

[20] R. J. H. Wesselink, K. Gupta, Z. Yuan, and P. J. Kelly, "Calculating spin transport properties from first principles: spin currents”, Phys. Rev. B 99, 144409 (2019).

[21] T. Valet and A. Fert, "Theory of the perpendicular magnetoresistance in magnetic multilayers", Phys. Rev. B 48, 7099-7113 (1993).

[22] S. Bandyopadhyay and M. Cahay, Introduction to Spintronics (CRC Press) (2008).

[23] R. J. Elliott, "Theory of the effect of spin-orbit coupling on magnetic resonance in some semiconductors", Phys. Rev. 96, 266-279 (1954).

[24] Y. Yafet, "g factors and spin-lattice relaxation of conduction electrons", in Solid State Physics, edited by F. Seitz and D. Turnbull, volume 14, 1-98 (Academic, New York) (1963).

[25] M. I. D’yakonov and V. I. Perel, "Possibility of orienting electron spins with current”, Zh. Eksp. Teor. Fiz. 13, 657-660 (1971), [JETP Letters-USSR 13, pp 467-469 (1971)].

[26] G. L. Bir, A. G. Aronov, and G. E. Pikus, "Spin relaxation of electrons scattered by holes”, Zh. Eksp. Teor. Fiz. 69, 1382-1397 (1975).

[27] N. F. Mott and H. S. W. Massey, The Theory of Atomic Collisions, 3 edition (Oxford Clarendon Press) (1949).

[28] Y. K. Kato, R. C. Myers, A. C. Gossard, and D. D. Awschalom, "Observation of the spin hall effect in semiconductors", Science 306, 1910-1913 (2004).

[29] J. Wunderlich, B. Kaestner, J. Sinova, and T. Jungwirth, "Experimental Observation of the Spin-Hall Effect in a Two-Dimensional Spin-Orbit Coupled Semiconductor System", Phys. Rev. Lett. 94, 047204 (2005). 
[30] L. Liu, C.-F. Pai, Y. Li, H. W. Tseng, D. C. Ralph, and R. A. Buhrman, "Spintorque switching with the giant spin Hall effect of tantalum", Science 336, 555-558 (2012).

[31] Y. Kim, S. H. Choday, and K. Roy, "DSH-MRAM: Differential Spin Hall MRAM for On-Chip Memories", IEEE Electron Device Letters 34, 1259-1261 (2013).

[32] K. Garello, C. O. Avci, I. M. Miron, M. Baumgartner, A. Ghosh, S. Auffret, O. Boulle, G. Gaudin, and P. Gambardella, "Ultrafast magnetization switching by spin-orbit torques", Appl. Phys. Lett. 105, 212402 (2014).

[33] L. Zhu, L. Zhu, S. Shi, M. Sui, D. C. Ralph, and R. A. Buhrman, "Enhancing SpinOrbit Torque by Strong Interfacial Scattering From Ultrathin Insertion Layers", Phys. Rev. Appl. 11, 061004 (2019).

[34] L. Wang, R. J. H. Wesselink, Y. Liu, Z. Yuan, K. Xia, and P. J. Kelly, "Giant Room Temperature Interface Spin Hall and Inverse Spin Hall Effects", Phys. Rev. Lett. 116, 196602 (2016).

[35] T. Tanaka, H. Kontani, M. Naito, T. Naito, D. S. Hirashima, K. Yamada, and J. Inoue, "Intrinsic spin Hall effect and orbital Hall effect in $4 d$ and $5 d$ transition metals", Phys. Rev. B 77, 165117 (2008).

[36] K. S. Das, W. Y. Schoemaker, B. J. van Wees, and I. J. Vera-Marun, "Spin injection and detection via the anomalous spin Hall effect of a ferromagnetic metal", Phys. Rev. B 96, 220408 (2017).

[37] S. C. Baek, V. P. Amin, Y.-W. Oh, G. Go, S.-J. Lee, G.-H. Lee, K.-J. Kim, M. D. Stiles, B.-G. Park, and K.-J. Lee, "Spin currents and spin-orbit torques in ferromagnetic trilayers", Nat. Mater. 17, 509-513 (2018).

[38] V. P. Amin, J. Li, M. D. Stiles, and P. M. Haney, "Intrinsic spin currents in ferromagnets", Phys. Rev. B 99, 220405(R) (2019).

[39] Y. Omori, E. Sagasta, Y. Niimi, M. Gradhand, L. E. Hueso, F. Casanova, and Y. Otani, "Relation between spin Hall effect and anomalous Hall effect in $3 d$ ferromagnetic metals", Phys. Rev. B 99, 014403 (2019).

[40] M. B. Lifshits and M. I. Dyakonov, "Swapping Spin Currents: Interchanging Spin and Flow Directions", Phys. Rev. Lett. 103, 186601 (2009).

[41] C. O. Pauyac, M. Chshiev, A. Manchon, and S. A. Nikolaev, "Spin Hall and Spin Swapping Torques in Diffusive Ferromagnets", Phys. Rev. Lett. 120, 176802 (2018).

[42] A. Davidson, V. P. Amin, W. S. Aljuaid, P. M. Haney, and X. Fan, "Perspectives of electrically generated spin currents in ferromagnetic materials", Phys. Lett. A 384, 126228 (2020).

[43] N. Nagaosa, J. Sinova, S. Onoda, A. H. MacDonald, and N. P. Ong, "Anomalous Hall effect", Rev. Mod. Phys. 82, 1539-1592 (2010). 
[44] K. M. Schep and G. E. W. Bauer, "Universality of transport through dirty interfaces", Phys. Rev. Lett. 78, 3015-3018 (1997).

[45] K. M. Schep, J. B. A. N. van Hoof, P. J. Kelly, G. E. W. Bauer, and J. E. Inglesfield, "Interface resistances of magnetic multilayers", Phys. Rev. B 56, 10805-10808 (1997).

[46] M. D. Stiles and D. R. Penn, "Calculation of spin-dependent interface resistance", Phys. Rev. B 61, 3200-3202 (2000).

[47] K. Xia, P. J. Kelly, G. E. W. Bauer, I. Turek, J. Kudrnovský, and V. Drchal, "Interface resistance of disordered magnetic multilayers", Phys. Rev. B 63, 064407 (2001).

[48] K. Gupta, R. J. H. Wesselink, R. Liu, Z. Yuan, and P. J. Kelly, "Disorder Dependence of Interface Spin Memory Loss", Phys. Rev. Lett. 124, 087702 (2020).

[49] D. Gall, "Electron mean free path in elemental metals", J. Appl. Phys. 119, 085101 (2016).

[50] K. Fuchs, "The conductivity of thin metallic films according to the electron theory of metals", Proc. Camb. Phil. Soc. 34, 100-108 (1938).

[51] E. H. Sondheimer, "The mean free path of electrons in metals", Adv. Phys. 1, 1-42 (1952).

[52] L. Liu, R. A. Buhrman, and D. C. Ralph, "Review and analysis of measurements of the spin Hall effect in platinum", arXiv:1111.3702v3 (2011).

[53] O. Stejskal, A. Thiaville, J. Hamrle, S. Fukami, and H. Ohno, "Current distribution in metallic multilayers from resistance measurements", Phys. Rev. B 101, 235437 (2020).

[54] P. Hohenberg and W. Kohn, "Inhomogeneous electron gas", Phys. Rev. 136, B864B871 (1964).

[55] W. Kohn and L. J. Sham, "Self-consistent equations including exchange and correlation effects", Phys. Rev. 140, A1133-A1138 (1965).

[56] T. Ando, "Quantum point contacts in magnetic fields", Phys. Rev. B 44, 8017-8027 (1991).

[57] A. A. Starikov, Y. Liu, Z. Yuan, and P. J. Kelly, "Calculating the transport properties of magnetic materials from first-principles including thermal and alloy disorder, noncollinearity and spin-orbit coupling”, Phys. Rev. B 97, 214415 (2018).

[58] S. Datta, Electronic Transport in Mesoscopic Systems (Cambridge University Press, Cambridge) (1995).

[59] Y. Imry, Introduction to Mesoscopic Physics, 2 edition (Oxford University Press, Oxford) (2002).

[60] N. W. Ashcroft and N. D. Mermin, Solid State Physics (Holt-Saunders International Editions, Philadelphia) (1976). 
[61] Y. Liu, Z. Yuan, R. J. H. Wesselink, A. A. Starikov, M. van Schilfgaarde, and P. J. Kelly, "Direct method for calculating temperature-dependent transport properties", Phys. Rev. B 91, 220405(R) (2015).

[62] D. R. Lide, ed., CRC Handbook of Chemistry and Physics, 90th Edition (Internet Version 2010) (CRC Press/Taylor and Francis, Boca Raton, FL) (2009).

[63] M. Born and K.Huang, Dynamical theory of crystal lattices, first edition (The Oxford Clarendon Press) (1954).

[64] O. K. Andersen, "Linear methods in band theory", Phys. Rev. B 12, 3060-3083 (1975).

[65] H. L. Skriver, The LMTO Method: Muffin-tin Orbitals and Electronic Structure, Lecture Notes in Computer Science (Springer-Verlag) (1984).

[66] O. K. Andersen, "Linear methods in band theory", in The Electronic Structure of Complex Systems, edited by P. Phariseau and W. M. Temmerman, volume 113 of NATO Advanced Science Institutes Series, Series B, 11-66 (Plenum Press, New York and London) (1984).

[67] O. K. Andersen, Z. Pawlowska, and O. Jepsen, "Illustration of the linear-muffin-tinorbital tight-binding representation: Compact orbitals and charge density in Si", Phys. Rev. B 34, 5253-5269 (1986).

[68] S. Zhang, "Spin Hall Effect in the Presence of Spin Diffusion", Phys. Rev. Lett. 85, 393-396 (2000).

[69] A. G. Aronov, "Spin injection in metals and polarization of nuclei", JETP Lett. 24, 32-34 (1976), [Pis'ma Zh. Eksp. Teor. Fiz. 24, 37 (1976)].

[70] M. Johnson and R. H. Silsbee, "Interfacial Charge-Spin Coupling: Injection and Detection of Spin Magnetization in Metals", Phys. Rev. Lett. 55, 1790-1793 (1985).

[71] P. C. van Son, H. van Kempen, and P. Wyder, "Boundary Resistance of the Ferromagnetic-Nonferromagnetic Metal Interface”, Phys. Rev. Lett. 58, 2271-2273 (1987).

[72] P. C. van Son, H. van Kempen, and P. Wyder, "Reply to Comment by Mark Johnson and R. H. Silsbee", Phys. Rev. Lett. 60, 378-378 (1988).

[73] M. I. Dyakonov and V. I. Perel, "Current-induced spin orientation of electrons in semiconductors”, Phys. Lett. A 35, 459-460 (1971).

[74] S. O. Valenzuela and M. Tinkham, "Direct electronic measurement of the spin Hall effect”, Nature (London) 442, 176-179 (2006).

[75] E. Saitoh, M. Ueda, H. Miyajima, and G. Tatara, "Conversion of spin current into charge current at room temperature: Inverse spin-Hall effect", Appl. Phys. Lett. 88, 182509 (2006). 
[76] A. Hoffmann, "Spin Hall effects in metals", IEEE Trans. Magn. 49, 5172-5193 (2013).

[77] J. Sinova, S. O. Valenzuela, J. Wunderlich, C. H. Back, and T. Jungwirth, "Spin Hall effects", Rev. Mod. Phys. 87, 1213-1259 (2015).

[78] S. Bhatti, R. Sbiaa, A. Hirohata, H. Ohno, S. Fukami, and S. N. Piramanayagam, "Spintronics based random access memory: a review", Materials Today 20, 530 548 (2017).

[79] T. Kimura, Y. Otani, T. Sato, S. Takahashi, and S. Maekawa, "Room-Temperature Reversible Spin Hall Effect”, Phys. Rev. Lett. 98, 156601 (2007).

[80] C. F. Pai, L. Q. Liu, Y. Li, H. W. Tseng, D. C. Ralph, and R. A. Buhrman, "Spin transfer torque devices utilizing the giant spin Hall effect of tungsten", Appl. Phys. Lett. 101, 122404 (2012).

[81] K. Ando, S. Takahashi, K. Harii, K. Sasage, J. Ieda, S. Maekawa, and E. Saitoh, "Electric Manipulation of Spin Relaxation Using the Spin Hall Effect", Phys. Rev. Lett. 101, 036601 (2008).

[82] O. Mosendz, J. E. Pearson, F. Y. Fradin, G. E. W. Bauer, S. D. Bader, and A. Hoffmann, "Quantifying Spin Hall Angles from Spin Pumping: Experiments and Theory", Phys. Rev. Lett. 104, 046601 (2010).

[83] O. Mosendz, V. Vlaminck, J. E. Pearson, F. Y. Fradin, G. E. W. Bauer, S. D. Bader, and A. Hoffmann, "Detection and quantification of inverse spin Hall effect from spin pumping in permalloy/normal metal bilayers", Phys. Rev. B 82, 214403 (2010).

[84] L. Liu, T. Moriyama, D. C. Ralph, and R. A. Buhrman, "Spin-Torque Ferromagnetic Resonance Induced by the Spin Hall Effect”, Phys. Rev. Lett. 106, 036601 (2011).

[85] L. Vila, T. Kimura, and Y. Otani, "Evolution of the Spin Hall Effect in Pt Nanowires: Size and Temperature Effects", Phys. Rev. Lett. 99, 226604 (2007).

[86] Y. Liu, Z. Yuan, R. J. H. Wesselink, A. A. Starikov, and P. J. Kelly, "Interface Enhancement of Gilbert Damping from First Principles", Phys. Rev. Lett. 113, 207202 (2014).

[87] M. Nguyen, D. C. Ralph, and R. A. Buhrman, "Spin Torque Study of the Spin Hall Conductivity and Spin Diffusion Length in Platinum Thin Films with Varying Resistivity", Phys. Rev. Lett. 116, 126601 (2016).

[88] E. Sagasta, Y. Omori, M. Isasa, M. Gradhand, L. E. Hueso, Y. Niimi, Y. Otani, and F. Casanova, "Tuning the spin Hall effect of Pt from the moderately dirty to the superclean regime", Phys. Rev. B 94, 060412(R) (2016).

[89] V. P. Amin and M. D. Stiles, "Spin transport at interfaces with spin-orbit coupling: Formalism”, Phys. Rev. B 94, 104419 (2016). 
[90] J. Fabian and S. Das Sarma, "Phonon-Induced Spin Relaxation of Conduction Electrons in Aluminum", Phys. Rev. Lett. 83, 1211-1214 (1999).

[91] A. T. Burkov and M. V. Vedernikov, "High-temperature thermoelectric power and electrical resistivity of single-crystal rhenium and their relation with the electronic structure", Zh. Eksp. Teor. Fiz. 85, 1821-1825 (1983).

[92] R. W. Powell, R. P. Tye, and M. J. Woodman, "The thermal conductivity and electrical resistivity of polycrystalline metals of the platinum group and of single crystals of ruthenium", Journal of the Less-Common Metals 12, 1-10 (1966).

[93] R. S. Nair, E. Barati, K. Gupta, Z. Yuan, and P. J. Kelly, "Spin-Flip Diffusion Length in $5 d$ Transition Metal Elements: a First-Principles Benchmark", Phys. Rev. Lett. 126, 196601 (2021).

[94] M. Zwierzycki, P. A. Khomyakov, A. A. Starikov, K. Xia, M. Talanana, P. X. Xu, V. M. Karpan, I. Marushchenko, I. Turek, G. E. W. Bauer, G. Brocks, and P. J. Kelly, "Calculating scattering matrices by wave function matching", Phys. Status Solidi B 245, 623-640 (2008).

[95] O. K. Andersen, O. Jepsen, and D. Glötzel, "Canonical description of the band structures of metals", in Highlights of Condensed Matter Theory, edited by F. Bassani, F. Fumi, and M. P. Tosi, International School of Physics 'Enrico Fermi', Varenna, Italy, 59-176 (North-Holland, Amsterdam) (1985).

[96] O. K. Andersen, O. Jepsen, and M. Šob, "Linearized band structure methods", in Electronic Band Structure and its Applications, edited by M. Yussouff, volume 283 of Lecture Notes in Physics, 1-57 (Springer Berlin Heidelberg) (1987).

[97] E. Alerstam, "Anisotropic diffusive transport: Connecting microscopic scattering and macroscopic transport properties", Phys. Rev. E 89, 063202 (2014).

[98] J. Fabian and S. Das Sarma, "Spin Relaxation of Conduction Electrons in Polyvalent Metals: Theory and a Realistic Calculation”, Phys. Rev. Lett. 81, 5624-5627 (1998).

[99] G. Y. Guo, S. Murakami, T.-W. Chen, and N. Nagaosa, "Intrinsic Spin Hall Effect in Platinum: First-Principles Calculations”, Phys. Rev. Lett. 100, 096401 (2008).

[100] J. Qiao, J. Zhou, Z. Yuan, and W. Zhao, "Calculation of intrinsic spin Hall conductivity by Wannier interpolation", Phys. Rev. B 98, 214402 (2018).

[101] J. H. Ryoo, C.-H. Park, and I. Souza, "Computation of intrinsic spin Hall conductivities from first principles using maximally localized Wannier functions", Phys. Rev. B 99, 235113 (2019).

[102] P. A. Khomyakov, G. Brocks, V. Karpan, M. Zwierzycki, and P. J. Kelly, "Conductance calculations for quantum wires and interfaces: mode matching and Green functions", Phys. Rev. B 72, 035450 (2005).

[103] M. Isasa, E. Villamor, L. E. Hueso, M. Gradhand, and F. Casanova, "Temperature dependence of spin diffusion length and spin Hall angle in Au and Pt", Phys. Rev. B 91, 024402 (2015). 
[104] M. Isasa, E. Villamor, L. E. Hueso, M. Gradhand, and F. Casanova, "Erratum", Phys. Rev. B 92, 019905(E) (2015).

[105] C. Stamm, C. Murer, M. Berritta, J. Feng, M. Gabureac, P. M. Oppeneer, and P. Gambardella, "Magneto-Optical Detection of the Spin Hall Effect in Pt and W Thin Films", Phys. Rev. Lett. 119, 087203 (2017).

[106] C. Xiao, Y. Liu, Z. Yuan, S. A. Yang, and Q. Niu, "Temperature dependence of the side-jump spin Hall conductivity”, Phys. Rev. B 100, 085425 (2019).

[107] K. Fritz, S. Wimmer, H. Ebert, and M. Meinert, "Large spin Hall effect in an amorphous binary alloy", Phys. Rev. B 98, 094433 (2018).

[108] F. Freimuth, S. Blügel, and Y. Mokrousov, "Anisotropic Spin Hall Effect from First Principles”, Phys. Rev. Lett. 105, 246602 (2010).

[109] M. Morota, Y. Niimi, K. Ohnishi, D. H. Wei, T. Tanaka, H. Kontani, T. Kimura, and Y. Otani, "Indication of intrinsic spin Hall effect in $4 d$ and $5 d$ transition metals", Phys. Rev. B 83, 174405 (2011).

[110] E. M. Savitskii, P. Geld, V. E. Zinovev, N. B. Gorina, V. I. Sperelup, V. P. Polyakova, and A. L. Sokolov, "Transport properties of osmium single crystals at high temperatures", Phys. Status Solidi A 49, K117-K120 (1978).

[111] J. T. Schriempf, "Thermal and electrical resistivities of osmium from 2 to $20^{\circ} \mathrm{K}$ ", Solid State Commun. 6, 873-876 (1968).

[112] B. Xu and M. J. Verstraete, "First-principles study of transport properties in Os and OsSi”, Phys. Rev. B 87, 134302 (2013).

[113] P. B. Allen, "New method for solving Boltzmann's equation for electrons in metals", Phys. Rev. B 17, 3725-3734 (1978).

[114] A. Manchon, J. Železný, I. M. Miron, T. Jungwirth, J. Sinova, A. Thiaville, K. Garello, and P. Gambardella, "Current-induced spin-orbit torques in ferromagnetic and antiferromagnetic systems", Rev. Mod. Phys. 91, 035004 (2019).

[115] K. V. Shanavas, Z. S. Popović, and S. Satpathy, "Theoretical model for Rashba spinorbit interaction in $d$ electrons", Phys. Rev. B 90, 165108 (2014).

[116] Y. Tserkovnyak, A. Brataas, and G. E. W. Bauer, "Enhanced Gilbert Damping in Thin Ferromagnetic Films", Phys. Rev. Lett. 88, 117601 (2002).

[117] Y. Tserkovnyak, A. Brataas, and G. E. W. Bauer, "Spin pumping and magnetization dynamics in metallic multilayers", Phys. Rev. B 66, 224403 (2002).

[118] Y. Tserkovnyak, A. Brataas, G. E. W. Bauer, and B. I. Halperin, "Nonlocal magnetization dynamics in ferromagnetic nanostructures", Rev. Mod. Phys. 77, 1375-1421 (2005). 
[119] K. Uchida, S. Takahashi, K. Harii, J. Ieda, W. Koshibae, K. Ando, S. Maekawa, and E. Saitoh, "Observation of the spin seebeck effect", Nature (London) 455, 778-781 (2008).

[120] K. Uchida, J. Xiao, H. Adachi, J. Ohe, S. Takahashi, J. Ieda, T. Ota, Y. Kajiwara, H. Umezawa, H. Kawai, G. E. W. Bauer, S. Maekawa, and E. Saitoh, "Spin Seebeck insulator", Nat. Mater. 9, 894-897 (2010).

[121] T. Taniguchi, J. Grollier, and M. D. Stiles, "Spin-transfer torques generated by the anomalous hall effect and anisotropic magnetoresistance", Phys. Rev. Appl. 3, 044001 (2015).

[122] B. F. Miao, S. Y. Huang, D. Qu, and C. L. Chien, "Inverse Spin Hall Effect in a Ferromagnetic Metal”, Phys. Rev. Lett. 111, 066602 (2013).

[123] A. Azevedo, O. Alves Santos, G. A. F. Guerra, R. O. Cunha, R. L. Rodríguez-Suárez, and S. M. Rezende, "Competing spin pumping effects in magnetic hybrid structures", Appl. Phys. Lett. 104, 052402 (2014).

[124] A. Azevedo, O. Alves Santos, R. O. Cunha, R. L. Rodríguez-Suárez, and S. M. Rezende, "Addition and subtraction of spin pumping voltages in magnetic hybrid structures", Appl. Phys. Lett. 104, 152408 (2014).

[125] A. Tsukahara, Y. Ando, Y. Kitamura, H. Emoto, E. Shikoh, M. P. Delmo, T. Shinjo, and M. Shiraishi, "Self-induced inverse spin hall effect in permalloy at room temperature”, Phys. Rev. B 89, 235317 (2014).

[126] M. Weiler, J. M. Shaw, H. T. Nembach, and T. J. Silva, "Detection of the DC Inverse Spin Hall Effect Due to Spin Pumping in a Novel Meander-Stripline Geometry", IEEE Magnetics Letters 5, 1-4 (2014).

[127] H. Wang, C. Du, P. C. Hammel, and F. Yang, "Spin current and inverse spin Hall effect in ferromagnetic metals probed by $\mathrm{Y}_{3} \mathrm{Fe}_{5} \mathrm{O}_{12}$-based spin pumping", Appl. Phys. Lett. 104, 202405 (2014).

[128] C. Du, H. Wang, F. Yang, and P. C. Hammel, "Systematic variation of spin-orbit coupling with $d$-orbital filling: Large inverse spin Hall effect in $3 d$ transition metals", Phys. Rev. B 90, 140407(R) (2014).

[129] D. Tian, Y. Li, D. Qu, S. Y. Huang, X. Jin, and C. L. Chien, "Manipulation of pure spin current in ferromagnetic metals independent of magnetization", Phys. Rev. B 94, 020403 (2016).

[130] C. Qin, S. Chen, Y. Cai, F. Kandaz, and Y. Ji, "Nonlocal electrical detection of spin accumulation generated by anomalous Hall effect in mesoscopic $\mathrm{Ni}_{81} \mathrm{Fe}_{19}$ films", Phys. Rev. B 96, 134418 (2017).

[131] S. Iihama, T. Taniguchi, K. Yakushiji, A. Fukushima, Y. Shiota, S. Tsunegi, R. Hiramatsu, S. Yuasa, Y. Suzuki, and H. Kubota, "Spin-transfer torque induced by the spin anomalous Hall effect”, Nat. Electronics 1, 120-123 (2018). 
[132] J. D. Gibbons, D. MacNeill, R. A. Buhrman, and D. Ralph, "Reorientable spin direction for spin current produced by the anomalous hall effect", Phys. Rev. Appl. 9, 064033 (2018).

[133] K. S. Das, J. Liu, B. J. van Wees, and I. J. Vera-Marun, "Efficient Injection and Detection of Out-of-Plane Spins via the Anomalous Spin Hall Effect in Permalloy Nanowires", Nano Letters 18, 5633-5639 (2018).

[134] M. W. Keller, K. S. Gerace, M. Arora, E. K. Delczeg-Czirjak, J. M. Shaw, and T. J. Silva, "Near-unity spin Hall ratio in $\mathrm{Ni}_{x} \mathrm{Cu}_{1-x}$ alloys", Phys. Rev. B 99, 214411 (2019).

[135] O. Gladii, L. Frangou, A. Hallal, R. L. Seeger, P. Noël, G. Forestier, S. Auffret, M. Rubio-Roy, P. Warin, L. Vila, S. Wimmer, H. Ebert, S. Gambarelli, M. Chshiev, and V. Baltz, "Self-induced inverse spin Hall effect in ferromagnets: Demonstration through nonmonotonic temperature dependence in permalloy", Phys. Rev. B 100, 174409 (2019).

[136] T. Taniguchi, "Magnetoresistance originated from charge-spin conversion in ferromagnet", AIP Advances 8, 055916 (2018).

[137] O. Šipr, S. Wimmer, S. Mankovsky, and H. Ebert, "Transport properties of doped permalloy via ab initio calculations: Effect of host disorder", Phys. Rev. B 101, 085109 (2020).

[138] N. E. Christensen, "Spin-orbit projected $d$ densities-of-states of Pd, Ag, Pt, and Au", J. Phys. F: Met. Phys. 8, L51-L55 (1978).

[139] D. Haskel, G. Fabbris, M. Zhernenkov, P. P. Kong, C. Q. Jin, G. Cao, and M. van Veenendaal, "Pressure Tuning of the Spin-Orbit Coupled Ground State in $\mathrm{Sr}_{2} \mathrm{IrO}_{4}$ ", Phys. Rev. Lett. 109, 027204 (2012).

[140] L. J. Swartzendruber, V. P. Itkin, and C. B. Alcock, "The Fe-Ni (Iron-Nickel) system", J. Phase Equilibria 12, 288-312 (1991).

[141] R. Karplus and J. M. Luttinger, "Hall effect in ferromagnetics", Phys. Rev. 95, 1154 1160 (1954).

[142] L. Berger, "Side-jump mechanism for the hall effect of ferromagnets", Phys. Rev. B 2, 4559-4566 (1970).

[143] S.-Q. Shen, "Spin Hall effect and Berry phase in two-dimensional electron gas", Phys. Rev. B 70, 081311(R) (2004).

[144] J. Kessler, Polarized Electrons, Theoretical and Mathematical Physics, 1 edition (Springer-Verlag, Berlin Heidelberg) (1976).

[145] P. G. Burke, Potential Scattering in Atomic Physics, 138, 1 edition (Plenum Press, New York) (1977).

[146] S. Zhang, P. M. Levy, and A. Fert, "Mechanisms of Spin-Polarized Current-Driven Magnetization Switching", Phys. Rev. Lett. 88, 236601 (2002). 
[147] O. K. Andersen and O. Jepsen, "Explicit, First-Principles Tight-Binding Theory", Phys. Rev. Lett. 53, 2571-2574 (1984).

[148] P. Soven, "Coherent-potential model of substitutional disordered alloys", Phys. Rev. 156, 809-813 (1967).

[149] I. Turek, V. Drchal, J. Kudrnovský, M. Šob, and P. Weinberger, Electronic Structure of Disordered Alloys, Surfaces and Interfaces (Kluwer, Boston-London-Dordrecht) (1997).

[150] Y. Liu, A. A. Starikov, Z. Yuan, and P. J. Kelly, "First-principles calculations of magnetization relaxation in pure $\mathrm{Fe}, \mathrm{Co}$, and $\mathrm{Ni}$ with frozen thermal lattice disorder", Phys. Rev. B 84, 014412 (2011).

[151] J. Crangle and G. C. Hallam, "The magnetization of face-centred cubic and bodycentred cubic iron + nickel alloys", Proc. R. Soc. London, Ser. A 272, 119-132 (1963).

[152] M. D. Kuz'min, "Shape of Temperature Dependence of Spontaneous Magnetization of Ferromagnets: Quantitative Analysis", Phys. Rev. Lett. 94, 107204 (2005).

[153] A. A. Starikov, P. J. Kelly, A. Brataas, Y. Tserkovnyak, and G. E. W. Bauer, "Unified First-Principles Study of Gilbert Damping, Spin-Flip Diffusion and Resistivity in Transition Metal Alloys", Phys. Rev. Lett. 105, 236601 (2010).

[154] W. Wang, T. Wang, V. P. Amin, Y. Wang, A. Radhakrishnan, A. Davidson, S. R. Allen, T. J. Silva, H. Ohldag, D. Balzar, B. L. Zink, P. M. Haney, and X. Fan, "Anomalous spin-orbit torques in magnetic single-layer films", Nat. Nanotechnol. 14, 819-824 (2019).

[155] A. M. Humphries, T. Wang, E. R. J. Edwards, S. R. Allen, J. M. Shaw, H. T. Nembach, J. Q. Xiao, T. J. Silva, and X. Fan, "Observation of spin-orbit effects with spin rotation symmetry", Nature Communications 8, 911 (2017).

[156] V. P. Amin, J. Zemen, and M. D. Stiles, "Interface-Generated Spin Currents", Phys. Rev. Lett. 121, 136805 (2018).

[157] G. Qu, K. Nakamura, and M. Hayashi, "Magnetization direction dependent spin Hall effect in $3 d$ ferromagnets", Phys. Rev. B 102, 144440 (2020).

[158] L. B. Valdes, "Resistivity measurements on germanium for transistors", Proceedings of the Institute of Radio Engineers 42, 420-427 (1954).

[159] L. J. van der Pauw, "A method of measuring specific resistivity and Hall effect of discs of arbitrary shape", Philips Research Reports 13, 1-9 (1958).

[160] I. Miccoli, F. Edler, H. Pfnür, and C. Tegenkamp, "The 100th anniversary of the fourpoint probe technique: the role of probe geometries in isotropic and anisotropic systems", J. Phys.: Condens. Matter 27, 223201 (2015). 
[161] K. L. Chopra, L. C. Bobb, and M. H. Francombe, "Electrical Resistivity of Thin SingleCrystal Gold Films", J. Appl. Phys. 34, 1699-1702 (1963).

[162] A. F. Mayadas, M. Shatzkes, and J. F. Janak, "Electrical resistivity model for polycrystalline films - case of specular reflection at external surfaces", Appl. Phys. Lett. 14, 345-347 (1969).

[163] J. W. C. de Vries, "Temperature and thickness dependence of the resistivity of thin polycrystalline aluminium, cobalt, nickel, palladium, silver and gold films", Thin Solid Films 167, 25-32 (1988).

[164] W. Wu, S. H. Brongersma, M. Van Hove, and K. Maex, "Influence of surface and grain-boundary scattering on the resistivity of copper in reduced dimensions", Appl. Phys. Lett. 84, 2838-2840 (2004).

[165] J. S. Chawla and D. Gall, "Specular electron scattering at single-crystal $\mathrm{Cu}(001)$ surfaces”, Appl. Phys. Lett. 94, 252101 (2009).

[166] J. S. Chawla, F. Gstrein, K. P. O'Brien, J. S. Clarke, and D. Gall, "Electron scattering at surfaces and grain boundaries in $\mathrm{Cu}$ thin films and wires", Phys. Rev. B 84, 235423 (2011).

[167] S. Dutta, K. Sankaran, K. Moors, G. Pourtois, S. Van Elshocht, J. Bömmels, W. Vandervorst, Z. Tőkei, and C. Adelmann, "Thickness dependence of the resistivity of platinum-group metal thin films", J. Appl. Phys. 122, 025107 (2017).

[168] W. Steinhögl, G. Schindler, G. Steinlesberger, and M. Engelhardt, "Size-dependent resistivity of metallic wires in the mesoscopic range", Phys. Rev. B 66, 075414 (2002).

[169] D. Josell, S. H. Brongersma, and Z. Tőkei, "Size-dependent resistivity in nanoscale interconnects", Ann. Rev. Mat. Res. 39, 231-254 (2009).

[170] J. Barnaś, A. Fuss, R. E. Camley, P. Grünberg, and W. Zinn, "Novel magnetoresistance effect in layered magnetic structures: Theory and experiment", Phys. Rev. B 42, 8110-8120 (1990).

[171] A. Hoffmann and S. D. Bader, "Opportunities at the Frontiers of Spintronics", Phys. Rev. Appl. 4, 047001 (2015).

[172] J. M. Ziman, Electrons and Phonons (Oxford University Press, London) (1960).

[173] P. B. Allen, "Boltzmann theory and resistivity of metals", in Quantum Theory of Real Materials, edited by J. R. Chelikowsky and S. G. Louie, chapter 17, 219-250 (Kluwer, Boston) (1996).

[174] S. Y. Savrasov and D. Y. Savrasov, "Electron-phonon interactions and related physical properties of metals from linear-response theory”, Phys. Rev. B 54, 16487-16501 (1996). 
[175] Y. Zhou, S. Sreekala, P. M. Ajayan, and S. K. Nayak, "Resistance of copper nanowires and comparison with carbon nanotube bundles for interconnect applications using first principles calculations", J. Phys.: Condens. Matter 20, 095209 (2008).

[176] V. Timoshevskii, Y. Ke, H. Guo, and D. Gall, "The influence of surface roughness on electrical conductance of thin Cu films: An ab initio study", J. Appl. Phys. 103, 113705 (2008).

[177] Y. Ke, F. Zahid, V. Timoshevskii, K. Xia, D. Gall, and H. Guo, "Resistivity of thin Cu films with surface roughness", Phys. Rev. B 79, 155406 (2009).

[178] B. Feldman, S. Park, M. Haverty, S. Shankar, and S. T. Dunham, "Simulation of grain boundary effects on electronic transport in metals, and detailed causes of scattering", Appl. Phys. Lett. 95, 222101 (2009).

[179] N. Kharche, S. R. Manjari, Y. Zhou, R. E. Geer, and S. K. Nayak, "A comparative study of quantum transport properties of silver and copper nanowires using first principles calculations", J. Phys.: Condens. Matter 23, 085501 (2011).

[180] J. M. Roberts, A. P. Kaushik, and J. S. Clarke, "Resistivity of sub-30 nm Copper Lines", 341-343 (IEEE) (2015).

[181] S. L. T. Jones, A. Sanchez-Soares, J. J. Plombon, A. P. Kaushik, R. E. Nagle, J. S. Clarke, and J. C. Greer, "Electron transport properties of sub-3-nm diameter copper nanowires”, Phys. Rev. B 92, 115413 (2015).

[182] A. Sanchez-Soares, S. L. T. Jones, J. J. Plombon, A. P. Kaushik, R. E. Nagle, J. S. Clarke, and J. C. Greer, "Effect of strain, thickness, and local surface environment on electron transport properties of oxygen-terminated copper thin films", Phys. Rev. B 94, 155404 (2016).

[183] N. A. Lanzillo, "Ab Initio evaluation of electron transport properties of Pt, Rh, Ir, and Pd nanowires for advanced interconnect applications", J. Appl. Phys. 121, 175104 (2017).

[184] Y.-N. Zhao, S.-X. Qu, and K. Xia, "Influence of the surface structure and vibration mode on the resistivity of Cu films", J. Appl. Phys. 110, 064312 (2011).

[185] T. Zhou and D. Gall, "Resistivity scaling due to electron surface scattering in thin metal layers”, Phys. Rev. B 97, 165406 (2018).

[186] H. E. Camblong and P. M. Levy, "Novel results for quasiclassical linear transport in metallic multilayers", Phys. Rev. Lett. 69, 2835-2838 (1992).

[187] S. Zhang, P. M. Levy, and A. Fert, "Conductivity and magnetoresistance of magnetic multilayered structures”, Phys. Rev. B 45, 8689-8702 (1992).

[188] X.-G. Zhang and W. H. Butler, "Conductivity of metallic films and multilayers", Phys. Rev. B 51, 10085-10103 (1995). 
[189] M. S. P. Lucas, "Electrical conductivity of thin metallic films with unlike surfaces", J. Appl. Phys. 36, 1632-1635 (1965).

[190] A. F. Mayadas and M. Shatzkes, "Electrical-resistivity model for polycrystalline films: the case of arbitrary reflection at external surfaces", Phys. Rev. B 1, 1382-1389 (1970).

[191] P. S. Modenov and A. S. Parkhomenko, Affine Transformations, volume 1, chapter IV, 97-151 (Academic Press) (1965).

[192] D. V. Ahuja and S. A. Coons, "Interactive graphics in data processing: Geometry for construction and display", IBM Systems Journal 7, 188-205 (1968).

[193] P. Bamberg and S. Sternberg, A course in mathematics for students of physics (Cambridge University Press, Cambridge) (1991).

[194] P. Comninos, Mathematical and Computer Programming Techniques for Computer Graphics (Springer-Verlag, London) (2006).

[195] Y.-D. Liang and B. A. Barsky, "A new concept and method for line clipping", ACM Trans. Graph. 3, 1-22 (1984).

[196] R. L. Panton, Streamfunctions and the Velocity Potential, chapter 12, 266-288, fourth edition (John Wiley \& Sons, Inc., Hoboken, New Jersey, U.S.A.) (2013).

[197] G. Wexler, "The size effect and the non-local Boltzmann transport equation in orifice and disk geometry”, Proc. Phys. Soc. 66, 927-941 (1966).

[198] H. L. Skriver and N. M. Rosengaard, "Ab initio work function of elemental metals", Phys. Rev. B 45, 9410-9412 (1992).

[199] H. L. Skriver and N. M. Rosengaard, "Surface energy and work function of elemental metals”, Phys. Rev. B 46, 7157-7168 (1992).

[200] G. H. O. Daalderop, P. J. Kelly, and M. F. H. Schuurmans, "Magnetic anisotropy of a free-standing Co monolayer and of multilayers which contain Co monolayers", Phys. Rev. B 50, 9989-10003 (1994).

[201] R. S. Nair, E. Barati, K. Gupta, Z. Yuan, and P. J. Kelly, "Spin-Flip Diffusion Length in $5 d$ Transition Metal Elements: a First-Principles Benchmark", Phys. Rev. Lett. 126, 196601 (2021).

[202] M. S. Rang, R. S. Nair, and P. J. Kelly, "A first principles study of transport through thin Pt films", to be published (2021).

[203] K. Ando, S. Takahashi, J. Ieda, Y. Kajiwara, H. Nakayama, T. Yoshino, K. Harii, Y. Fujikawa, M. Matsuo, S. Maekawa, and E. Saitoh, "Inverse spin-Hall effect induced by spin pumping in metallic system", J. Appl. Phys. 109, 103913 (2011).

[204] Y. Niimi, M. Morota, D. H. Wei, C. Deranlot, M. Basletic, A. Hamzic, A. Fert, and Y. Otani, "Extrinsic Spin Hall Effect Induced by Iridium Impurities in Copper", Phys. Rev. Lett. 106, 126601 (2011). 
[205] R. S. Nair and P. J. Kelly, "Fully resolved currents from quantum transport calculations", Phys. Rev. B 103, 195406 (2021).

[206] J. Sinova, D. Culcer, Q. Niu, N. A. Sinitsyn, T. Jungwirth, and A. H. MacDonald, "Universal intrinsic spin Hall effect", Phys. Rev. Lett. 92, 126603 (2004).

[207] A. Brataas, Y. Tserkovnyak, and G. E. W. Bauer, "Spin-pumping in ferromagnetnormal metal systems", J. Magn. Magn. Mater. 272, 1981 (2004).

[208] W. Zhang, V. Vlaminck, J. E. Pearson, R. Divan, S. D. Bader, and A. Hoffmann, "Determination of the Pt spin diffusion length by spin-pumping and spin Hall effect", Appl. Phys. Lett. 103, 242414 (2013).

[209] E. Sagasta, Y. Omori, M. Isasa, Y. Otani, L. E. Hueso, and F. Casanova, "Spin diffusion length of Permalloy using spin absorption in lateral spin valves", Appl. Phys. Lett. 111, 082407 (2017).

[210] G. Y. Guo, Y. Yao, and Q. Niu, "Ab initio Calculation of the Intrinsic Spin Hall Effect in Semiconductors", Phys. Rev. Lett. 94, 226601 (2005).

[211] V. P. Amin and M. D. Stiles, "Spin transport at interfaces with spin-orbit coupling: Phenomenology”, Phys. Rev. B 94, 104420 (2016).

[212] Y. Tserkovnyak, B. I. Halperin, A. A. Kovalev, and A. Brataas, "Boundary spin Hall effect in a two-dimensional semiconductor system with Rashba spin-orbit coupling", Phys. Rev. B 76, 085319 (2007).

[213] C. Sanz-Fernández, J. Borge, I. V. Tokatly, and F. S. Bergeret, "Nonlocal magnetolectric effects in diffusive conductors with spatially inhomogeneous spin-orbit coupling", Phys. Rev. B 100, 195406 (2019).

[214] G. Kästle, H.-G. Boyen, A. Schröder, A. Plettl, and P. Ziemann, "Size effect of the resistivity of thin epitaxial gold films", Phys. Rev. B 70, 165414 (2004).

[215] M. Cattani and M. C. Salvadori, "Electrical resistivity of platinum and gold thin films: a theoretical approach", Surface Review and Letters 11, 283-290 (2004).

[216] Y. V. Sharvin, "A possible method for studying Fermi surfaces", Zh. Eksp. Teor. Fiz. 48, 984-985 (1965), [Sov. Phys. JETP 21, 655-656 (1965)].

[217] R. Liu, K. Gupta, Z. Yuan, and P. J. Kelly, "Calculating the spin memory loss at $\mathrm{Cu|Metal}$ interfaces from first principles", To be published.

[218] K. Gupta, R. S. Nair, Z. Yuan, and P. J. Kelly, "Large spin current generation in bulk Fe-Ni systems", In preparation (2020).

[219] E. Villamor, M. Isasa, L. E. Hueso, and F. Casanova, "Contribution of defects to the spin relaxation in copper nanowires”, Phys. Rev. B 87, 094417 (2013).

[220] K.-W. Kim and K.-J. Lee, "Generalized Spin Drift-Diffusion Formalism in the Presence of Spin-Orbit Interaction of Ferromagnets”, Phys. Rev. Lett. 125, 207205 (2020). 
[221] A. M. Humphries, T. Wang, E. R. J. Edwards, S. R. Allen, J. M. Shaw, H. T. Nembach, J. Q. Xiao, T. J. Silva, and X. Fan, "Observation of spin-orbit effects with spin rotation symmetry", Nat. Commun. 8, 911 (2017).

[222] J. Smit, "The spontaneous Hall effect in ferromagnetics II", Physica 24, 39-51 (1958).

[223] E. Merzbacher, Quantum Mechanics, third edition (John Wiley \& Sons, New York) (1998).

[224] A. Fert and P. M. Levy, "Spin Hall Effect Induced by Resonant Scattering on Impurities in Metals", Phys. Rev. B 106, 157208 (2011).

[225] B. A. Lippmann, "Ehrenfest's theorem and scattering theory", Phys. Rev. Lett. 15, 11-14 (1965).

[226] O. P. Sushkov, A. I. Milstein, M. Mori, and S. Maekawa, "Relativistic effects in scattering of polarized electrons", Europhys. Lett. 103, 47003 (2013).

[227] P. Nozières and C. Lewiner, "A simple theory of the anomalous Hall effect in semiconductors", J. de Physique (Paris) 34, 901-915 (1973). 


\section{Summary}

I studied a number of topics in the field of electronic spin transport in transition metals. Because of their partially filled $d$ bands and complex Fermi surfaces with spin-orbit coupling, transition metals are rich in spin phenomena that have potential for spintronics applications. Using a density functional theory based scattering approach, I studied the generation and diffusion of spin currents in non-magnetic $5 d$ and ferromagnetic $3 d$ transition metals. By including thermal disorder I presented realistic calculations for these metals. I next studied lateral transport in finite size geometries that are encountered in spintronics experiments. This form the last three chapters of the thesis. The main focus of the thesis has been on the determination of the two most important spin-orbit coupling related spin transport properties, the spin flip diffusion length, $l_{\mathrm{sf}}$ and the spin Hall angle, $\Theta_{\mathrm{sH}}$.

I began by addressing the issue of the growing discrepancies in the values of $l_{\mathrm{sf}}$ and $\Theta_{\mathrm{sH}}$ reported for the Pt group of metals in Chapter 2. In this chapter I presented benchmark values for $l_{\mathrm{sf}}$ in bulk $5 d$ transition metals as a function of the temperature. The products $l_{\mathrm{sf}} \times \rho$ and $l_{\mathrm{sf}} \times \Theta_{\mathrm{sH}}$ were found to be approximately constant for these materials. The former confirms the validity of the Elliott-Yafet mechanism as the dominant mechanism of spin relaxation in $5 d$ transition metals. A major takeaway is that $l_{\mathrm{sf}}$ is inversely proportional to the electron density of states at the Fermi level. This may make it possible to tune $l_{\mathrm{sf}}$ by alloying. The complex interplay between various band structure parameters in determining the values of $l_{\mathrm{sf}}$ and $\Theta_{\mathrm{sH}}$ has been revealed by this study.

Using the same scattering calculations I studied the spin Hall effect in $\mathrm{Ni}_{x} \mathrm{Fe}_{1-x}$ alloys by explicitly calculating the transverse currents excited by an applied charge current. In Chapter 3, I demonstrated that the spin Hall effect observed in these materials is comparable to that in Pt. A study of the concentration dependence concluded that pure Ni has the largest value of $\Theta_{\mathrm{sH}}$ at room temperature, approximately $7.5 \%$ compared to $4.2 \%$ in Pt. An intuitive theoretical framework based on Mott scattering was derived to identify the various intrinsically generated current components and how they interact with the magnetization of the ferromagnet. Skew scattering was found to be the dominant contribution to the spin Hall and spin swapping angles. 
In Chapter 4, I presented a "fully resolved" current scheme that makes it possible to study transport in all three spatial dimensions with atomic resolution. Using this scheme I calculated how charge currents are distributed in thin films of Pt and $\mathrm{Au}$ as well as in periodic Pt|Au and Pt|Ir multilayers. I demonstrated the validity of the scheme by calculating the flow of charge currents in two limits, "classical" and "Knudsen" determined by the ratio of the thickness of the film to the mean free path of the material. My method made it possible to visualize the classical phenomenon of charge shunting on an atomic level.

Using the fully resolved current scheme, I calculated the spin Hall effect in a thin film of Pt and presented the numerical results and a phenomenological model with which to interpret them in Chapter 5. I was able to perform calculations for thicknesses of approximately $6 l_{\mathrm{sf}}^{\mathrm{Pt}}$ which allowed me to study the diffusion of a spin current towards the surface in a bulk-like sample. An existing model by Zhang et al. [68] which is universally applied to interpret experiments including recent MOKE experiments [105] did not agree with my calculated results. The analysis led to the finding that a surface spin Hall angle is needed to correctly describe the calculations. I derived an improved model based on Valet-Fert theory by including the surface spin Hall angle $\Theta_{S}^{\perp}$. The surface contribution to the spin current flowing parallel to the surface as $\Theta_{S}^{\|}$was also quantified.

Finally, in Chapter 6, I presented the results of calculations of the spin Hall effect in non-magnetic $\mathrm{Pt} \mid \mathrm{Au}$ and ferromagnetic $\mathrm{Py}(\mathrm{Ni}) \mid \mathrm{Cu}$ multilayers. I obtained injection efficiencies of around 50\% across $\mathrm{Pt} \mid \mathrm{Au}$ as well as Py|Cu interfaces and approximately $20 \%$ across the $\mathrm{Ni} \mid \mathrm{Cu}$ interface. The latter point towards a larger value of the spin memory loss at the $\mathrm{Ni} \mid \mathrm{Cu}$ interface compared to the other interfaces. By incorporating the interface into the Valet-Fert theory, a model to describe the results calculated for Pt|Au multilayer was derived. Using this model I extracted the spin memory loss parameter $\delta$ and the interface spin Hall angle $\Theta_{\mathrm{I}}$ for the non-magnetic interface. A semiclassical description of the injection of spin Hall currents through a $\mathrm{Py}(\mathrm{Ni}) \mid \mathrm{Cu}$ interface requires carefully accounting for additional transport parameters introduced by the magnetization of the ferromagnet. The computational results highlight the need to extend the model I presented for a non-magnetic multilayer to include ferromagnetic materials and to devise a scheme for extracting all important spin transport parameters required to predict lateral spin injection by the spin Hall effect. 


\section{Acknowledgements}

This thesis was not built in a day; some acknowledgements are due. Many have directly and indirectly helped me bring my efforts to fruition.

I am indebted to Paul for giving me, a mechanical engineer, the opportunity to pursue a $\mathrm{PhD}$ in condensed matter physics. He made it possible for me to segue from engineering to physics. I have greatly benefited from his immense knowledge in the subject and curiosity to understand my calculations in detail. He taught me to keep the reader in mind while writing a manuscript and has spent the painstaking effort to literally dot the i's and cross the t's in the manuscripts I wrote. He has always been available for discussions even on weekends after I started my new job, helping me finish the thesis on time. I express my deep sense of gratitude towards him and wish him a happy retirement with his family.

I express my sincere appreciation to all my collaborators who enthusiastically contributed at different stages of my research. I thank Kriti for the continued support in the works that culminated in Chapters 2 and 3. I am thankful to Zhe for his stimulating visit to the University of Twente a few years ago and for his valuable inputs to several chapters in this thesis. As a newcomer, it was easing to share the office with Ehsan, I thank him for the contributions to Chapter 2 of this thesis. It was a great pleasure to share the office with Max during the last phase of my $\mathrm{PhD}$ journey. Chapters 4 and 5 have greatly improved from his input and I am keen to continue our active collaboration. I wish him luck on his $\mathrm{PhD}$ journey..

It has been an immense pleasure working with my colleagues at the University of Twente. I thank Geert for his humorous and informative coffee chats, what a pity that I couldn't attend any of his popular lectures. I thank Sridevi for her kind help on numerous occasions, Yuqiang for the interesting conversations and quick responses to my queries and Monica for the delightful interactions. I am grateful to our former secretaries Els and Nicole for their support on everything administrative.

My heartfelt thanks to all the friends I made during the journey for the colourful times. I remain grateful to my parents Ajitha and Sasidharan for lovingly supporting me from the other end of the world. I am gratified to have made my parents proud by successfully finishing my $\mathrm{PhD}$. I thank my sister Reshmi and her family for cheering me all the way. I am filled with gratitude to my partner Inge whom I met along the way. She took care of me and kept my spirits high during the difficult times. A special thanks to her family for making me feel at home. 
I thank the graduation committee members for the review of the thesis. In particular, I thank Dr.Stiles for sending me detailed feedback on all the chapters.

Last but not least, I am thankful to Shell and NWO-I for materializing the CSER $\mathrm{PhD} 75$ program and funding my research. This has been a remarkable journey!

August 2021, Enschede 